\title{
Bio-inspired MEMS \\ Flow and Inertial Sensors
}
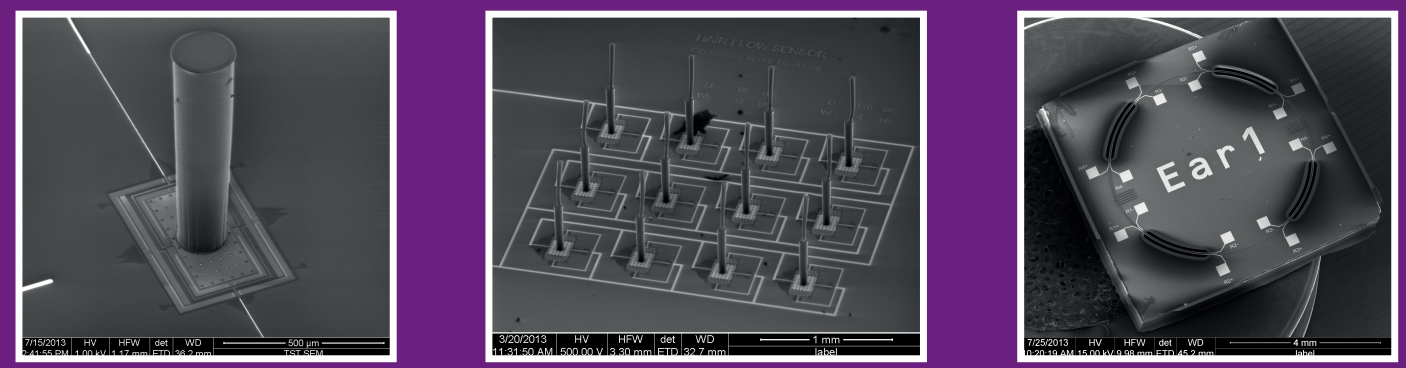

Harmen Droogendijk 


\section{BIO-INSPIRED MEMS FLOW AND INERTIAL SENSORS}

Harmen DroogendiJk 


\section{De promotiecommissie}

\section{Voorzitter en secretaris}

Prof. dr. P. M. G. Apers

Universiteit Twente

\section{Promotor}

Prof. dr. ir. G. J. M. Krijnen

Universiteit Twente

\section{Leden}

Prof. dr. J. Casas

Université de Tours (FR)

Prof. dr. D. Robert

University of Bristol (UK)

Prof. dr. E. J. Stamhuis

Prof. dr. ir. P. H. Veltink

Rijksuniversiteit Groningen

Prof. dr. ir. A. de Boer

Universiteit Twente

Universiteit Twente
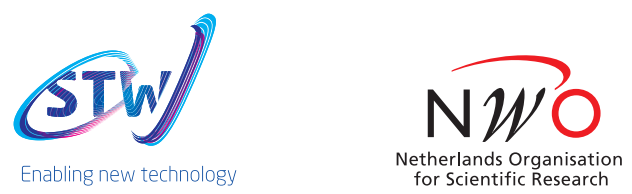

MESA+

\section{UNIVERSITY OF TWENTE.}

The research described in this dissertation is part of the Bio-inspired Engineering of ARray Sensors (BioEARS) project and has been conducted at the chair of Transducers Science and Technology of the department of Electrical Engineering at the University of Twente. This research is supported by the Dutch Technology Foundation STW, which is part of the Netherlands Organisation for Scientific Research (NWO) and partly funded by the Ministry of Economic Affairs (project number 07197).

Cover design by Harmen Droogendijk.

Printed by Gildeprint Drukkerijen, Enschede, the Netherlands.

Typeset with LATEX.

(C) Harmen Droogendijk, Enschede, the Netherlands, 2014.

ISBN 978-90-365-3598-4

DOI $10.3990 / 1.9789036535984$ 


\title{
BIO-INSPIRED MEMS FLOW AND INERTIAL SENSORS
}

\section{PROEFSCHRIFT}

\author{
ter verkrijging van \\ de graad van doctor aan de Universiteit Twente, \\ op gezag van de rector magnificus, \\ prof. dr. H. Brinksma, \\ volgens besluit van het College voor Promoties \\ in het openbaar te verdedigen \\ op vrijdag 28 februari 2014 om 14.45 uur
}

door

Harmen Droogendijk

geboren op 11 januari 1985

te Tholen 
Dit proefschrift is goedgekeurd door:

Prof. dr. ir. G. J. M. Krijnen Universiteit Twente (promotor) 
Aan mijn ouders Jan en Wina, aan mijn vrouw Klaske,

en aan mijn kinderen Lieneke en Ruben 



\section{Abstract}

In biology, mechanosensors, equipped with differing hair-like structures for signal pick-up, are sensitive to a variety of physical quantities like acceleration, flow, rotational rate, balancing and IR-light. As an example, crickets use filiform hairs for sensing of low-frequency flows to obtain information about the environment and avoid e.g. predator attacks crickets. Their filiform hairs are able to sense airflows with velocity amplitudes down to $30 \mu \mathrm{m} \mathrm{s}^{-1}$ and operate around the energy levels of thermal noise. Taking these hair-sensors as a source of inspiration, hair-sensor inspired flow-sensors for measurement of (tiny) ac-airflows using capacitive readout have been designed and fabricated using technology generally denoted as MEMS (microelectromechanical systems). To determine the performance of these flow sensitive hair-sensors, three different setups for oscillatory airflow are used for thorough characterization. Each of these flow sources has specific properties regarding frequency range, pressure dependence and bandwidth. By combining information from the used flow setups important insights in the sensor operation are gained and discussed.

To improve the performance of these hair flow sensors, the nature of energybuffering two-port transducers is exploited for implementation of electrostatic spring softening (ESS). On the application of a dc-bias voltage on the capacitors of our flow sensors, both an increase in responsivity for frequencies within the sensor's bandwidth and a lower flow velocity threshold are obtained. Changing the dc-bias voltage to an ac-bias voltage, non-resonant parametric amplification and filtering has been demonstrated in our hair flow sensors. By selecting appropriate values for the ac-bias voltage, selective gain and filtering is achieved. On application of an appropriate sinusoidal voltage on the capacitor plates, upconversion of the flow information is achieved when the flow frequency is much lower than the voltage frequency resulting in electromechanical amplitude modulation (EMAM). It is demonstrated that EMAM can improve the measurement performance at low frequencies, in case of limitations within the measurement setup. This method can be applied equally well to transients as to harmonic signals.

Under certain conditions, noise can be used to increase signal-to-noise ratio by 
exploiting the concept of stochastic resonance (SR). This concept is demonstrated using a voltage-controlled MEMS-slider, the signal-to-noise ratio can be increased by adding white noise. SR is implemented by controlling the strength of positiondependent capacitive wells by a dc-bias voltage, operating the device in push-pull mode by electrostatic actuation and adding a judiciously amount of white noise to the actuation comb drives. It is demonstrated that the use of SR allows for detection of sub-threshold forces and that the noise bandwidth has a clear impact on the required optimal noise strength.

Further, three different types of bio-inspired inertial sensors have been developed. First, a biomimetic accelerometer has been realized using surface micromachining and SU-8 lithography, inspired by the clavate hair system of the cricket. Second, inspired by the fly's haltere, a biomimetic gimbal-suspended hair-based gyroscope has been designed, fabricated and partially characterized. Third, an angular accelerometer based on the semicircular channels of the vestibular system has been developed. The accelerometer consists of a water-filled tube, wherein the fluid flow velocity is measured thermally as a representative for the external angular acceleration. For all three sensors, the necessary models are presented and guidelines are derived for optimization. Also, their performance is compared to their biological counterpart and its biomimetic potential is discussed.

In quantifying the performance of our MEMS hair flow sensors and comparing it to their source of inspiration, five independent metrics and a figure of merit are described, modelled and evaluated for both cricket and MEMS hair sensors. In general, cricket flow sensors perform not only better than the MEMS hair sensors, but are also close to operation at their physical limits. The results emphasize the intriguing research on bio-inspired sensors in order to learn from nature. 


\section{Contents}

$\begin{array}{ll}\text { Abstract } & \text { i }\end{array}$

Contents

1 Introduction 1

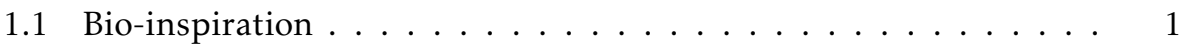

1.1.1 Cricket's filiform hairs . . . . . . . . . . . . . . 1

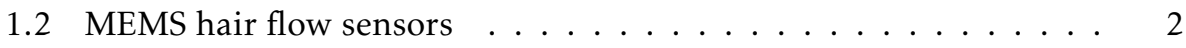

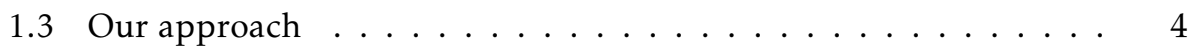

1.4 Aim of the research ................. 8

1.4.1 Performance optimization ............ 8

1.4 .2 Hair flow sensors . . . . . . . . . . . . . 10

1.4.3 Bio-inspired sensors ................. 10

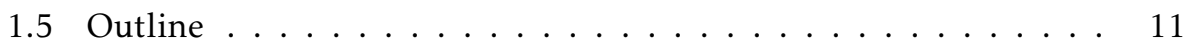

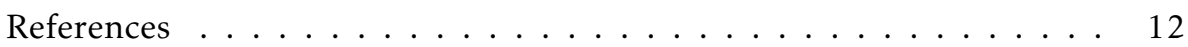

2 Characterization $\quad 15$

2.1 Introduction . . . . . . . . . . . . . . 15

2.2 Theory and modelling . . . . . . . . . . . . 15

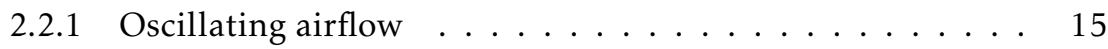

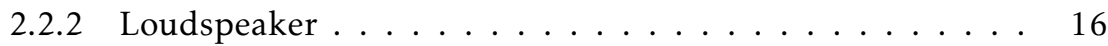

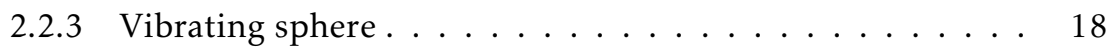

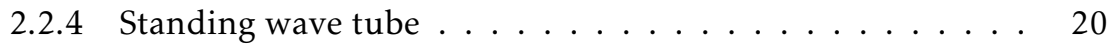

2.3 Bio-inspired hair flow sensors . . . . . . . . . . . . 22

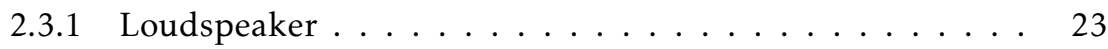

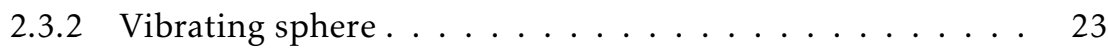

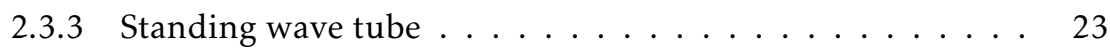

2.4 Discussion ........................... 24

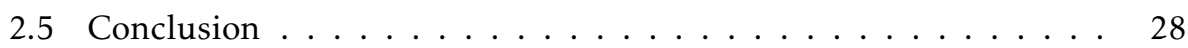

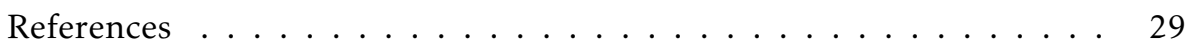


3 Adaptation by tunable stiffness 31

3.1 Introduction . . . . . . . . . . . . . . . . 31

3.2 Electrostatic spring softening . . . . . . . . . . 32

3.2.1 Virtual dc-biasing . . . . . . . . . . . . 35

3.2.2 Impact on mechanical response . . . . . . . . . . . 36

3.2.3 Noise and detection threshold . . . . . . . . . . . 37

3.3 Electrostatic spring hardening . . . . . . . . . . . . . 38

3.4 Experimental . . . . . . . . . . . . . . . . . 41

3.4.1 Electrostatic spring softening . . . . . . . . . . . 41

3.4.2 Electrostatic spring hardening . . . . . . . . . . . 45

3.5 Discussion . . . . . . . . . . . . . . . . . 48

3.5.1 Electrostatic spring softening . . . . . . . . . . . 48

3.5.2 Electrostatic spring hardening . . . . . . . . . . . . . . . 49

3.5 .3 Overall ...................... 50

3.6 Conclusions ......................... 50

References ......................... 51

4 Non-resonant parametric amplification 53

4.1 Introduction . . . . . . . . . . . . . . . 53

4.2 Theory and modelling . . . . . . . . . . . . . 54

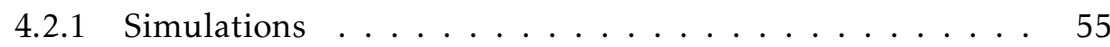

4.2.2 Harmonic balancing method . . . . . . . . . . . 55

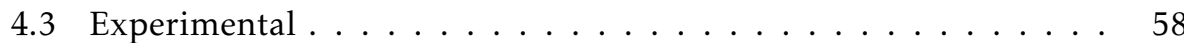

4.3.1 Impact of ESH on NRPA . . . . . . . . . . 61

4.4 Discussion ........................ 63

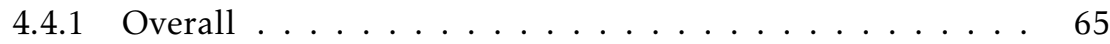

4.5 Conclusions ......................... 65

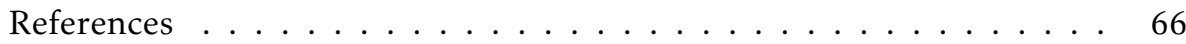

5 Electromechanical amplitude modulation $\quad 69$

5.1 Introduction . . . . . . . . . . . . . . . . . 69

5.2 Theory and modelling . . . . . . . . . . . . 70

5.2 .1 Hair mechanics . . . . . . . . . . . . . 71

5.2.2 Stiffness modulation ............... 72

5.2.3 Electromechanical amplitude modulation . . . . . . . 73

5.3 Experimental ....................... 79

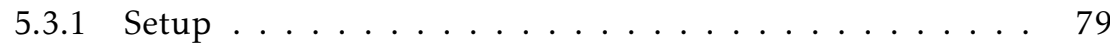

5.3 .2 Demonstration of EMAM . . . . . . . . . . 80

5.3.3 Improving measurement quality . . . . . . . . . 81 
5.3.4 Application of EMAM to transient flows . . . . . . . . . 82

5.4 Discussion ..................... 86

5.4 .1 Noise analysis . . . . . . . . . . . . 86

5.4.2 Influence of higher frequency components . . . . . . . . 87

5.4.3 EMAM with electronic read-out . . . . . . . . . . . 88

5.4 .4 Extension of the concept . . . . . . . . . 88

5.5 Conclusions ........................ 88

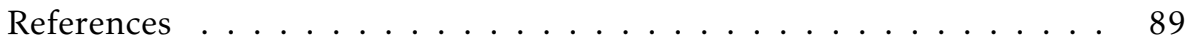

6 Stochastic resonance in a micromechanical slider 91

6.1 Introduction . . . . . . . . . . . . . . . . . 91

6.2 Theory and modelling . . . . . . . . . . . . 93

6.2.1 Stochastic resonance ............... 93

6.2 .2 Energy function . . . . . . . . . . . . . 95

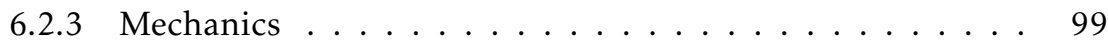

6.2 .4 Signal-to-noise ratio . . . . . . . . . . 100

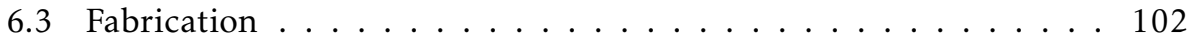

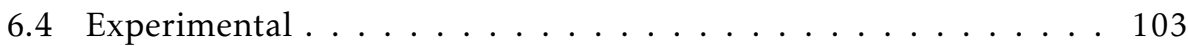

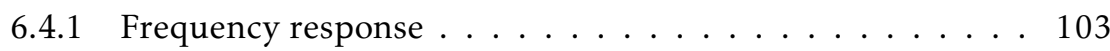

6.4 .2 SR-experiments . . . . . . . . . . . 105

6.4 .3 Noise bandwidth ................ 106

6.4 .4 Non-sinusoidal waveforms . . . . . . . . . . . . . 107

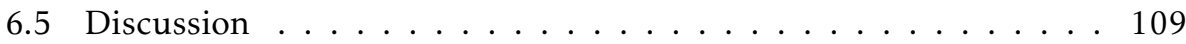

6.5 .1 Advantage of SR . . . . . . . . . . . . . . 109

6.5 .2 Waveforms ................... 110

6.5 .3 Noise bandwidth . . . . . . . . . . . . . 111

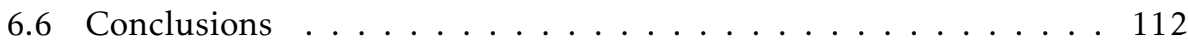

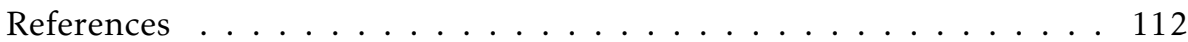

$\begin{array}{llr}7 & \text { Biomimetic accelerometer } & 117\end{array}$

7.1 Introduction . . . . . . . . . . . . . . 117

7.2 Theory and modelling . . . . . . . . . . . . . . . 119

7.2.1 Hair mechanics . . . . . . . . . . . . . . 119

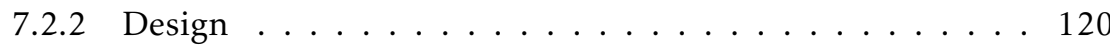

7.2.3 Flow contributions . . . . . . . . . . . . 121

7.2 .4 Thermal noise . . . . . . . . . . . . 122

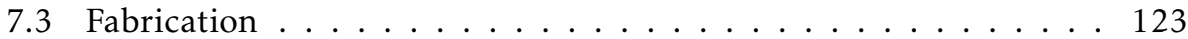

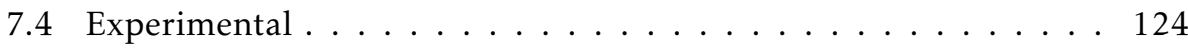

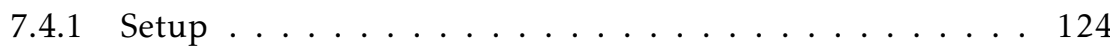


7.4.2 Frequency response . . . . . . . . . . . . 126

7.4.3 Directivity ................... 127

7.4.4 Threshold and linearity . . . . . . . . . . . . 127

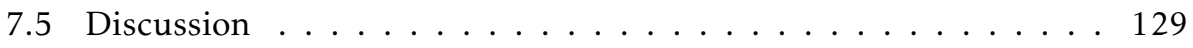

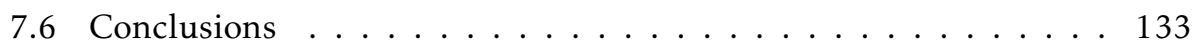

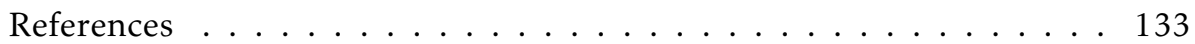

8 Biomimetic gyroscope 137

8.1 Introduction . . . . . . . . . . . . . . 137

8.2 Theory and modelling . . . . . . . . . . . . . 139

8.2.1 Mechanics . . . . . . . . . . . . . . . . . 139

8.2 .2 Design rules . . . . . . . . . . . . . . 141

8.2 .3 Thermal noise . . . . . . . . . . . . . 145

8.2 .4 Design .................... 147

8.3 Fabrication ....................... 148

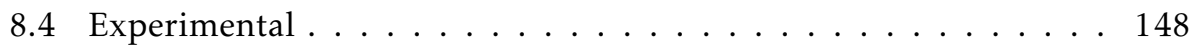

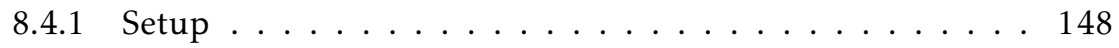

8.4.2 Frequency response . . . . . . . . . . . 150

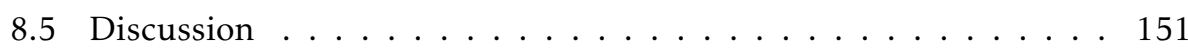

8.5 .1 Fabrication ....................... 151

8.5.2 Modal response . . . . . . . . . . . . . . 152

8.5 .3 Biomimetic approach . . . . . . . . . . . 153

8.5.4 Comparison to the fly's haltere . . . . . . . . . . 154

8.6 Conclusions . . . . . . . . . . . . . . . . . . 155

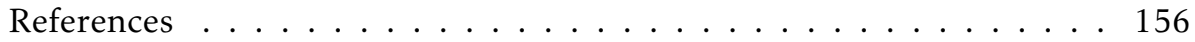

9 Bio-inspired angular accelerometer $\quad 161$

9.1 Introduction . . . . . . . . . . . . . . . 161

9.2 Theory and modelling . . . . . . . . . . . . . . . 162

9.2 .1 Fluid dynamics . . . . . . . . . . . . . . . . . 162

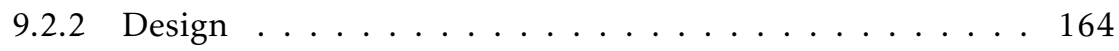

9.3 Fabrication . . . . . . . . . . . . . . . 165

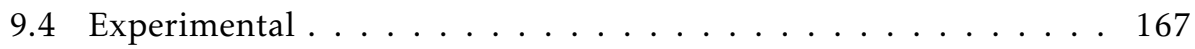

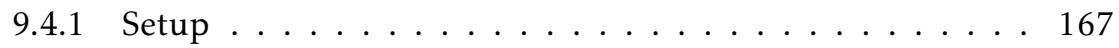

9.4 .2 Measurements . . . . . . . . . . . . . 167

9.5 Discussion . . . . . . . . . . . . . . . . 169

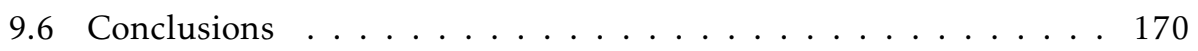

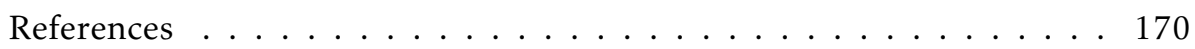


10 Performance assessment of flow sensing hairs 173

10.1 Introduction . . . . . . . . . . . . . . . . 173

10.1.1 Bio-inspired flow sensors . . . . . . . . . . . . 174

10.2 Hair mechanical model . . . . . . . . . . . . . . . . . 177

10.3 Performance metrics . . . . . . . . . . . . . . . 182

10.3.1 Responsivity . . . . . . . . . . . . . 182

10.3.2 Power transfer . . . . . . . . . . . . . . . 183

10.3 .3 Power efficiency . . . . . . . . . . . . . . . . . . . . . . . . . . . . . . . . . . . . . . . .

10.3.4 Response time . . . . . . . . . . . . . . 185

10.3 .5 Detection threshold . . . . . . . . . . . . . 186

10.3.6 Figure of merit . . . . . . . . . . . . . . 187

10.4 Application of metrics . . . . . . . . . . . . . 188

10.5 Discussion . . . . . . . . . . . . . . . . 189

10.5.1 Model evaluation and choice of performance metrics . . . . 191

10.5.2 Unwanted cross-talk between sensory modalities and multifunctional sensory systems . . . . . . . . . . . . 193

10.5.3 Improving MEMS flow sensors: time to abandon natureinspired design? . . . . . . . . . . . . . . . 194

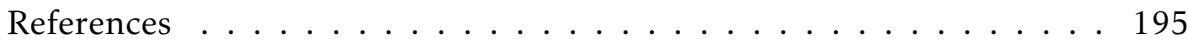

11 Conclusions and outlook 201

11.1 Conclusions ....................... 201

11.1.1 Performance optimization . . . . . . . . . . . 201

11.1.2 Hair flow sensors . . . . . . . . . . . . . 202

11.1.3 Bio-inspired sensors ... . . . . . . . . 203

11.2 Outlook . . . . . . . . . . . . . . . . . 204

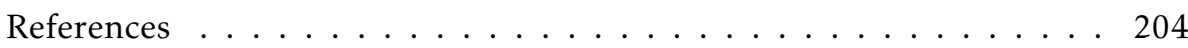

A Oscillating airflow and Stokes' drag coefficient 205

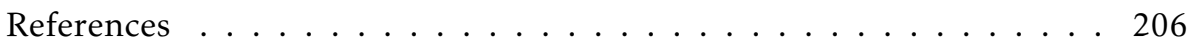

B Loudspeaker transduction model 207

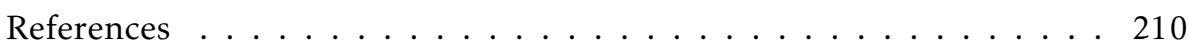

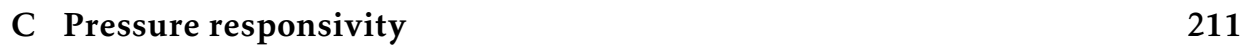

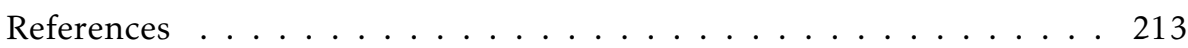

D Capacitive read-out $\quad 215$

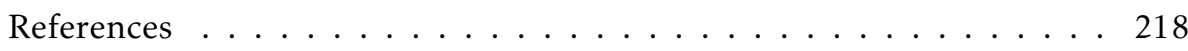


E Process flow for hair sensors $\quad 219$

E.1 Introduction . . . . . . . . . . . . . . . . . . . . . 219

E.2 Specific design properties ................ 219

E.2.1 Design rules ...................... 219

E.2.2 Masks ..................... 220

E.2.3 Flat SiRN membrane for springs and membranes . . . . . . 220

E.3 Experimental details . . . . . . . . . . . . . . 221

E.3.1 Trench filling and thinning by oxidation of poly-silicon . . 221

E.3.2 Sacrificial layer etching by $\mathrm{XeF}_{2} \ldots \ldots . \ldots 224$

E.4 Process outline . . . . . . . . . . . . . . 226

E.5 Process parameters . . . . . . . . . . . . . . . . 229

E.5.1 Mask inspection . . . . . . . . . . . . . . 229

E.5.2 Substrate selection . . . . . . . . . . . . . . . 229

E.5.3 Patterning of the device layer - SOI . . . . . . . . . . 229

E.5.4 LPCVD of $\mathrm{Si}_{3} \mathrm{~N}_{4} \ldots \ldots \ldots . \ldots . \ldots 232$

E.5.5 LPCVD of poly-Si and annealing . . . . . . . . . . . 234

E.5.6 Partial oxidation of polysilicon . . . . . . . . . 235

E.5.7 Patterning of the device layer - I (SACRI) . . . . . . . . . 236

E.5.8 Thermal oxidation - I . . . . . . . . . . . . . . 238

E.5.9 LPCVD of SiRN (membranes and springs) . . . . . . . . . . 239

E.5.10 SiRN patterning (membranes, springs and bottom electrode contact) . . . . . . . . . . . . . . 240

E.5.11 Thermal oxidation - II . . . . . . . . . . . . . . 242

E.5.12 LPCVD of SiRN (support beams) . . . . . . . . . . . . . 243

E.5.13 SiRN patterning (support beams) . . . . . . . . . . . 244

E.5.14 Sputtering of Al . . . . . . . . . . . . . . . . 246

E.5.15 Patterning of $\mathrm{Al}$ - Membrane . . . . . . . . . . . . 247

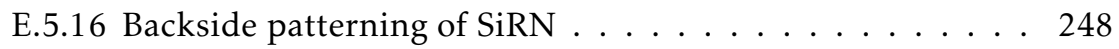

E.5.17 SU-8 hairs . . . . . . . . . . . . . . . . . 250

E.5.18 Sacrificial layer etching . . . . . . . . . . . . 252

F Process flow for MEMS sliders 253

F.1 Introduction . . . . . . . . . . . . . . . 253

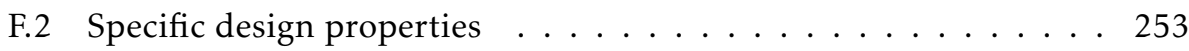

F.2.1 Design rules . . . . . . . . . . . . . . 253

F.2.2 Masks..................... 254

F.3 Process outline . . . . . . . . . . . . . . 255

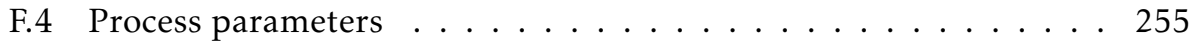

F.4.1 Mask inspection . . . . . . . . . . . . 255 


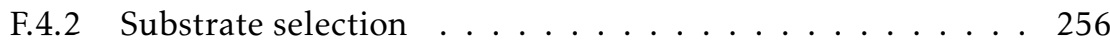

F.4.3 Patterning of the device layer - SOI . . . . . . . . . 256

F.4.4 Dicing for breaking grooves . . . . . . . . . . . . . . 259

F.4.5 Release by vapour HF . . . . . . . . . . . . . . . 260

$\begin{array}{ll}\text { Publications } & 263\end{array}$

Journal articles . . . . . . . . . . . . . . . . . 263

Conference contributions . . . . . . . . . . . . . . . . 264

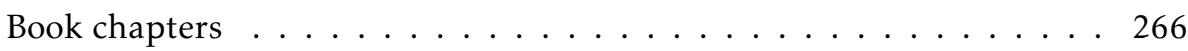

Miscellaneous . . . . . . . . . . . . . . . . . . . 266

$\begin{array}{ll}\text { Samenvatting } & 269\end{array}$

$\begin{array}{ll}\text { Woord van dank } & 271\end{array}$

$\begin{array}{ll}\text { Biografie } & 275\end{array}$ 


\section{1 \\ Introduction}

\subsection{Bio-inspiration}

Biology displays a variety of sensory mechanisms that constitute exceptional sensory performance, e.g. with respect to sensitivity, dynamic range, frequency filtering and selectivity. Auditory sensing systems in nature may exhibit mechanical filtering and amplification (White and Grosh, 2005), whereas flow sensing systems may make use of noise to enhance their sensing capabilities (Levin and Miller, 1996). Such systems and their natural implementation form a rich source of inspiration to engineers. Despite advancements in engineering and technology throughout history, it is still challenging for engineered systems to compete with biological systems. For example, the auditory capabilities of bats to perceive their environment, locate prey and to navigate at high velocities through complex surroundings (e.g. with leafed brushes and trees) (Schnitzler et al., 2003) has no engineered equivalent.

\subsubsection{Cricket's filiform hairs}

Crickets are capable of sensing low-frequency sound by using mechanoreceptive sensory hairs to obtain information about the environment and avoid e.g. predator 


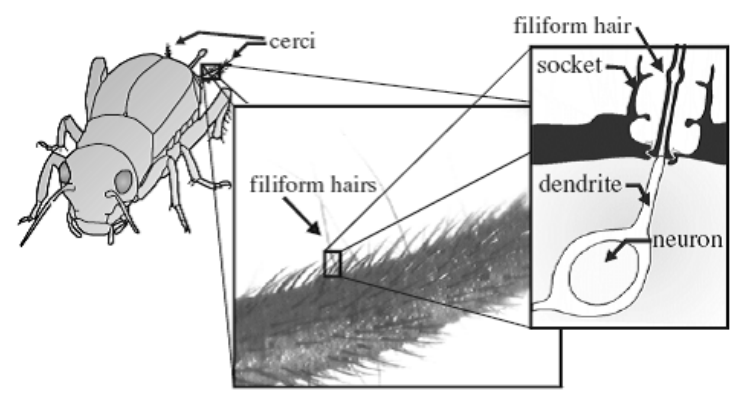

Figure 1.1: Flow perception by crickets using mechanoreceptor filiform hairs.

attacks (Dangles et al., 2005). These so-called filiform hairs, which are situated on the back of the cricket's body on appendices called cerci, are able to sense airflows with velocity amplitudes down to $30 \mu^{-1} \mathrm{~m} \mathrm{~s}^{-1}$ (Shimozawa et al., 1998) and operate around the energy levels of thermal noise (Shimozawa et al., 2003). Each hair is lodged in a socket, guiding the hair to move in a preferred direction. When subjected to airflow, the neuron is fired upon rotation of the hair base (figure 1.1). Indicatively, for wood crickets (Nemobius sylvestris) the hairs vary in length up to around $1 \mathrm{~mm}$, with a bimodal distribution with concentrations around 150 and $750 \mu \mathrm{m}$ (Dangles et al., 2005).

\subsection{MEMS hair flow sensors}

The cricket's filiform hair dimensions allow, in principle, for biomimicry by a technology generally denoted as MEMS (microelectromechanical systems). Taking these hair-sensors as a source of inspiration, several research groups have worked on the development of artificial counterparts for airflow measurements by exploiting MEMS technology (figure 1.2).

Measurement of dc-airflows using hair-sensor inspired flow sensors was shown by Ozaki et al. (2000). They realized artificial hair-sensors by fabrication of cantilevers with read-out by strain gauges, and measured flow velocities ranging from tens of $\mathrm{cm} \mathrm{s}^{-1}$ up to $2 \mathrm{~m} \mathrm{~s}^{-1}$. Other groups (Tao and $\mathrm{Yu}, 2012$ ) also developed cantilever-based structures with strain gauges for measurement of dc-airflows, and showed measurements of flow velocities ranging from $0.7 \mathrm{~mm} \mathrm{~s}^{-1}$ up to $20 \mathrm{~m} \mathrm{~s}^{-1}$ (Chen et al., 2007) and up to of $45 \mathrm{~m} \mathrm{~s}^{-1}$ (Wang et al., 2007). Sadeghi et al. (2011) fabricated an artificial hair flow sensor by manually mounting a hair on a hydraulic sensor-system, for conversion of angular rotation into capacitive changes, capable of measuring dc-flows from (theoretically) $3 \mathrm{~mm} \mathrm{~s}^{-1}$ up to $10 \mathrm{~m} \mathrm{~s}^{-1}$. Inspired by 


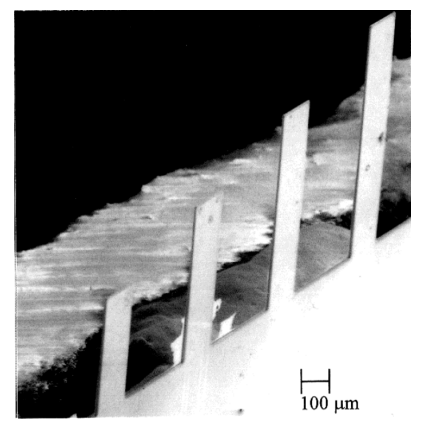

(a) Cantilever-based flow sensor with strain gauges (Ozaki et al., 2000).

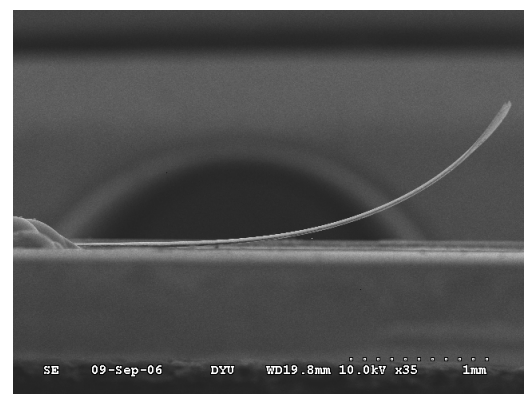

(c) Flow sensor with a $\mathrm{Si}_{3} \mathrm{~N}_{4}$ cantilever and strain gauges (Wang et al., 2007).

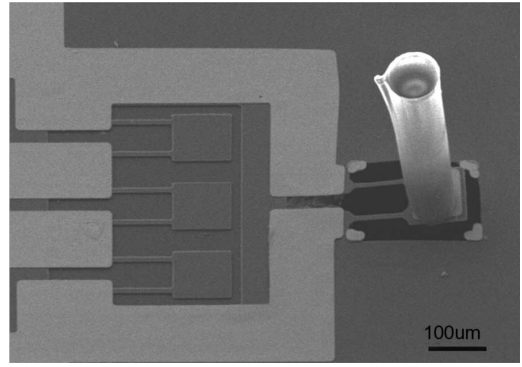

(b) Piezo-resistive flow sensor with a PDMA hair (Chen et al., 2007).

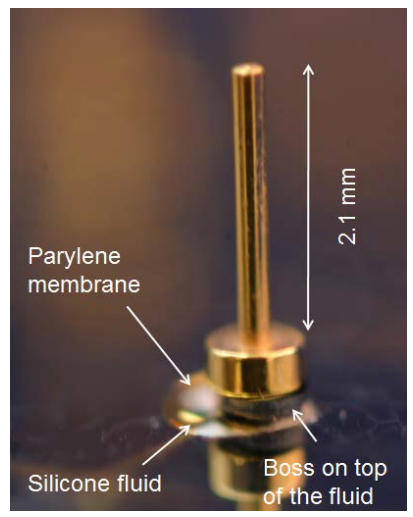

(d) Hydraulic sensor-system with capacitive read-out (Sadeghi et al., 2011).

Figure 1.2: Previously developed artificial hair flow sensors.

distributed arrays of flow sensitive hair receptors as found on bat wings, the application of hair sensors in boundary-layer separation detection for flight control has been theoretically investigated by Dickinson (2010) with respect to sensing approaches (but no sensor developments were reported).

Flow sensors based on the lateral line of fish have been developed by Fan et al. (2002), and were shown to measure water flows ranging up to $1 \mathrm{~m} \mathrm{~s}^{-1}$. Chen et al. (2007) developed an artificial hair-cell sensor to perform measurements in water, with a detection limit below $1 \mathrm{~mm} \mathrm{~s}^{-1}$ for a $50 \mathrm{~Hz}$ oscillatory water flow measured in $2 \mathrm{~Hz}$ bandwidth. Improvement in sensitivity of hair-sensors for aquatic use by a factor of 40 due to capping of the hair by a hydro-gel is reported by McConney et al. (2009). Research on combining several flow sensors for realization of an artificial lateral line canal for hydrodynamic detection has been carried out by 
Yang et al. (2011).

Triggered by biologically as well as physically motivated questions regarding the density of hair-sensor arrays in crickets, the viscosity mediated coupling between hairs has been investigated by various groups theoretically (Bathellier et al., 2005), numerically (Lewin and Hallam, 2010) (Cummins et al., 2007) and experimentally (Bathellier et al., 2005; Alagirisamy et al., 2009; Casas et al., 2010).

\subsection{Our approach}

In contrast, hair-sensor inspired flow sensors for measurement of (tiny) ac-airflows using capacitive read-out have been designed and fabricated in our group during the past ten years. As a main part of the hair flow sensory system, an artificial hair, fabricated by means of MEMS technology, is required. The first generation of artificial hairs based on silicon rich nitride using narrow trenches in a silicon substrate was demonstrated by van Baar et al. (2003), allowing for different hair lengths and shapes (figure 1.3a). The hair fabrication process was improved by Dijkstra et al. (2005), by using SU-8 as a base material. A schematic view of this sensor structure is shown in figure 1.4.

The SU-8 flow-susceptible hair is positioned on a SiRN-membrane, which forms, by virtue of a thin chromium layer, also the upper plate of the capacitive structure. The bulk silicon below acts as a bottom contact, allowing for (differential) capacitive read-out of the hair rotation angle and is thus a measure for the incoming airflow. The poly-Si shown in the schematic view is used a sacrificial layer, for achieving a rotatable membrane. Arrays of hairs were successfully fabricated (figure 1.3b) and their functional response was demonstrated.

By increasing the hair length using a double-spun and exposed SU-8 layer (figure 1.3c) and improving the overall fabrication process and design (figure 1.3d), the current performance of our cricket-inspired hair flow sensors is limited by electronic-noise, enabling the detection and measurement of flow velocities in the range of sub-mm/s while retaining a bandwidth on the order of $1 \mathrm{kHz}$ (Bruinink et al., 2009). Additionally, we have demonstrated the use of arrays of hair-sensors by changing the wafer-type to silicon-on-insulator (SOI) and isolation trenches (Dagamseh et al., 2010) and their suitability as so-called 'flow cameras' (Dagamseh et al., 2012).

An overview of the current fabrication process (appendix E) for the biomimetic hair flow sensors is shown in figure 1.5. The sensor is fabricated on a siliconon-insulator wafer (I). Trenches are etched in the silicon device layer using DRIE. A layer of $200 \mathrm{~nm}$ stoichiometric $\mathrm{Si}_{3} \mathrm{~N}_{4}$ is used for covering and protecting 


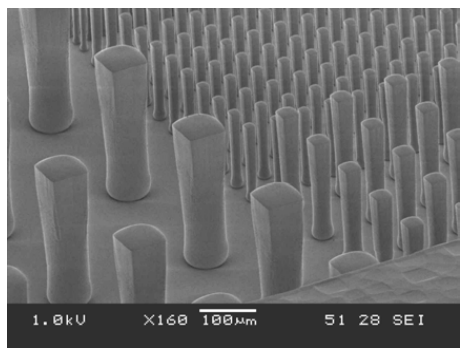

(a) Close-up of SiRN hairs of different size (van Baar et al., 2003).

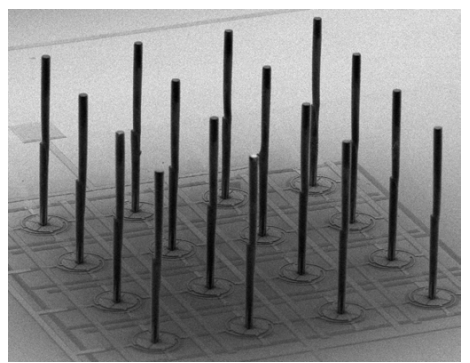

(c) Arrays of hairs of double-spun and exposed SU-8 layers (Krijnen et al., 2006).

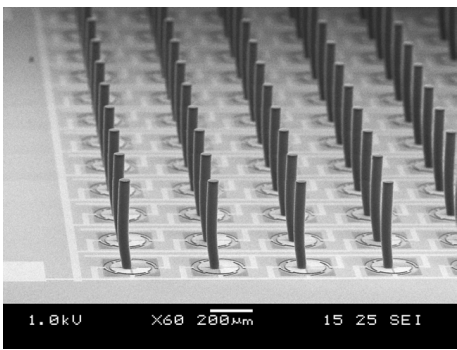

(b) Array of spiral-suspended sensory SU8 hairs. (Dijkstra et al., 2005).

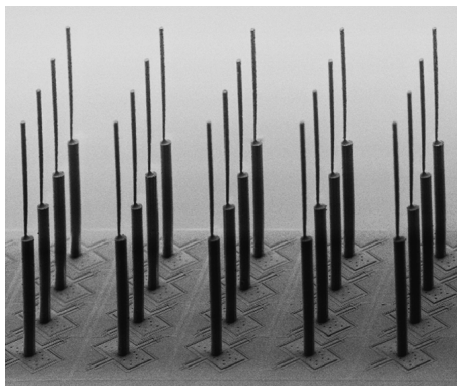

(d) Part of an array with $900 \mu \mathrm{m}$ long SU8 hairs (Bruinink et al., 2009).

Figure 1.3: MEMS hair flow sensors fabricated in our group by surface micromachining.

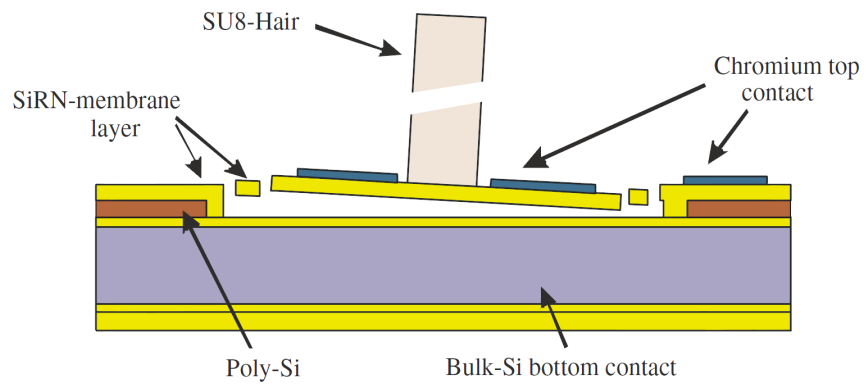

Figure 1.4: Schematic view of the sensor structure with SU-8 hair (Krijnen et al., 2006).

the trenches (II). The device layer contains two electrodes, which are used for capacitive readout of the flow-induced movement. On top of the $\mathrm{Si}_{3} \mathrm{~N}_{4}$ layer, a sacrificial layer of poly-silicon is deposited by LPCVD. Two wet oxidation runs are necessary for smoothing the trench fillings and to reduce the poly-silicon layer thickness down to $600 \mathrm{~nm}$ (III). 


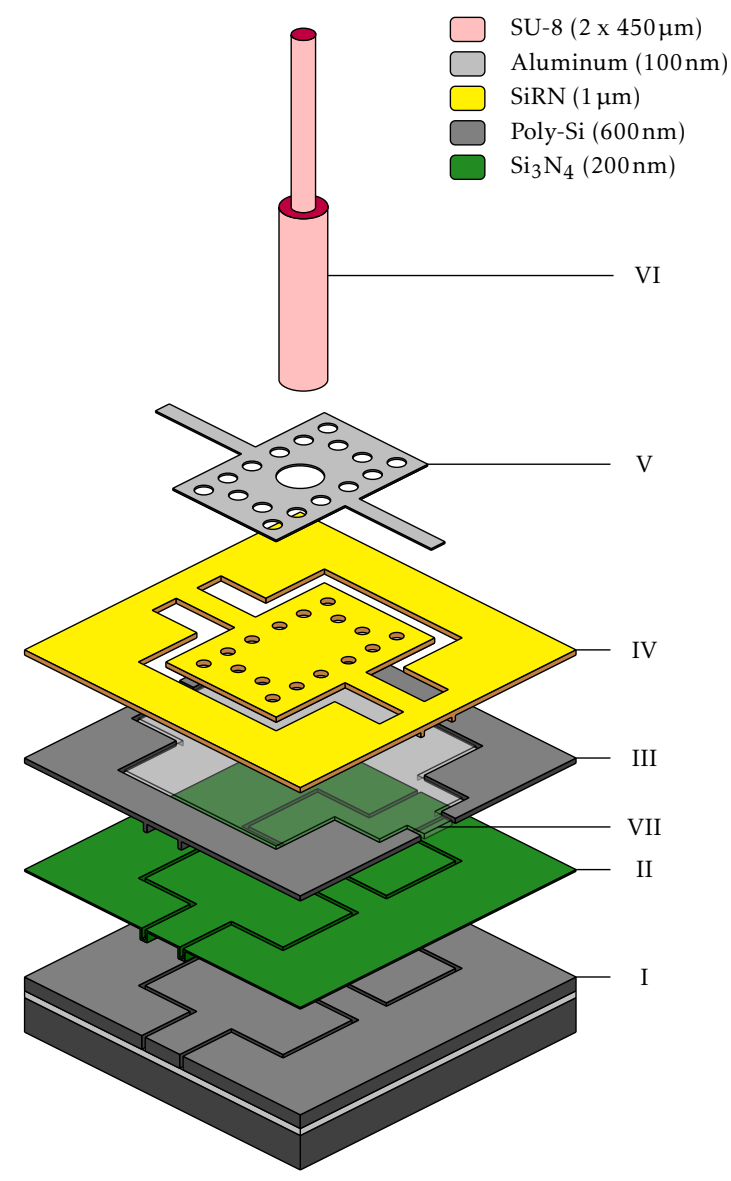

Figure 1.5: Schematic representation of the artificial hair flow sensor fabrication process (Dagamseh et al., 2013).

The sensor membrane and springs are constructed by depositing and patterning a $1 \mu \mathrm{m} \mathrm{SiRN} \mathrm{layer} \mathrm{on} \mathrm{top} \mathrm{of} \mathrm{the} \mathrm{poly-silicon} \mathrm{(IV).} \mathrm{Aluminum}(100 \mathrm{~nm})$ is sputtered on top of the membrane to create the electrodes for capacitive readout $(\mathrm{V})$. Our artificial filiform hair is created by two layers of SU-8, to realize both the centre of mass towards the bottom of the hair structure (low moment of inertia). The total hair length is about $900 \mu \mathrm{m}$, whereas the lower diameter is about $50 \mu \mathrm{m}$ and the upper diameter approximately $25 \mu \mathrm{m}$ (VI). Finally, to release the membrane the sacrificial poly-silicon layer is removed using $\mathrm{XeF}_{2}$ etching (VII).

Using a loudspeaker for generation of sinusoidal airflows, the frequency 


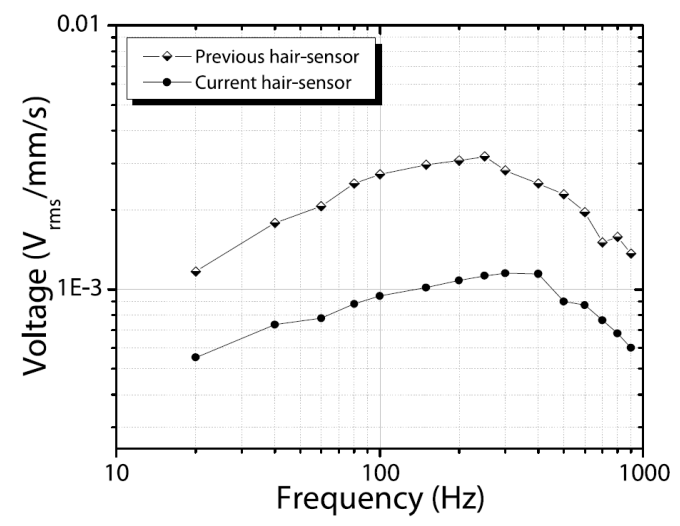

Figure 1.6: Frequency response measurements of the arrayed hair flow sensor compared with the single hair flow sensor normalized to an airflow of $1 \mathrm{~mm} \mathrm{~s}^{-1}$ (Dagamseh et al., 2013).

response of the artificial hair flow sensors was determined (Dagamseh et al., 2013). The airflow amplitude was measured using a commercially available particle velocity sensor (Microflown (de Bree, 2003)). For differential capacitive measurement of the hair rotational angle, two $1 \mathrm{MHz}$ signals $180^{\circ}$ out of phase where used, thereby implementing amplitude modulation of the rotational angle. This angle is resolved by demodulation using synchronous detection, filtered by a $3 \mathrm{kHz}$ low-pass filter and measured with a digital multimeter.

The measured frequency response is shown in figure 1.6. Here, the measured responses are shown for the 'previous' artificial hair flow sensors fabricated by Bruinink et al. (2009) and the 'current' artificial hair flow sensors fabricated by Dagamseh et al. (2013). Both sensors have an identical mechanical design and were measured by the same interfacing electronics. The results show similarities between the frequency responses of both sensors. However, the measured 'current' hair flow sensor was a so-called single hair sensor, whereas the 'previous' hair flow sensor consisted of an array of 124 hairs.

As a measure for the sensitivity of the artificial hair flow sensors, measurements were conducted for finding their detection limit. This limit is defined as the airflow amplitude at which the sensor response has a signal-to-noise ratio equal to unity. The measured response for a range of airflow velocity amplitudes of $0.1-50 \mathrm{~mm} \mathrm{~s}^{-1}$ with a flow frequency of $250 \mathrm{~Hz}$ is shown in figure 1.7. Here, the detection limit is indicated by the intersection of the extrapolated sensor response with the line of constant noise.

The response for both the 'previous' and 'current' hair flow sensor are shown in 


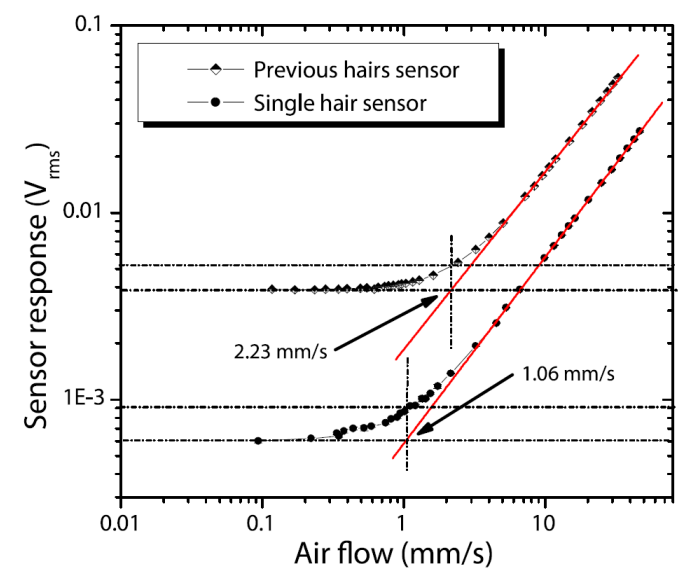

Figure 1.7: Voltage response of both the arrayed hair flow sensor and the single hair flow sensor as a function of the flow velocity amplitude at $250 \mathrm{~Hz}$ plotted together with the asymptotic lines (in red) to determine the threshold flow amplitude (Dagamseh et al., 2013).

figure 1.7, wherein the noise level of the single hair flow sensor is clearly reduced, compared to the array of hair flow sensors consisting of 124 hair flow sensors in parallel. Further, the single hair flow sensor shows a relative improvement of $52 \%$ in the threshold response down to an airflow amplitude of $1.06 \mathrm{~mm} \mathrm{~s}^{-1}$ measured in a bandwidth of $3 \mathrm{kHz}$.

Directivity measurements for both types of hair flow sensor are shown in figure 1.8 , together with an ideal figure-of-eight shaped directivity. In these measurements, the sensor was incrementally rotated in steps of $10^{\circ}$ with respect to the airflow source. Both measurements for the 'previous' hair flow sensors (figure 1.8a) and the 'current' hair flow sensor (figure 1.8b) indicate a clear response to airflow by exhibiting the theoretical figure-of-eight.

\subsection{Aim of the research}

\subsubsection{Performance optimization}

In nature, one can find a range of principles exploited to obtain outstanding sensitivity, dynamic range and selectivity (e.g. (Avitabile et al., 2010)). The aim of this research is to look carefully at the mechanisms that biological systems exploit to attain their outstanding sensory capabilities, like adaptation, filtering, (electro)mechanical amplification, stochastic resonance, and investigate their 


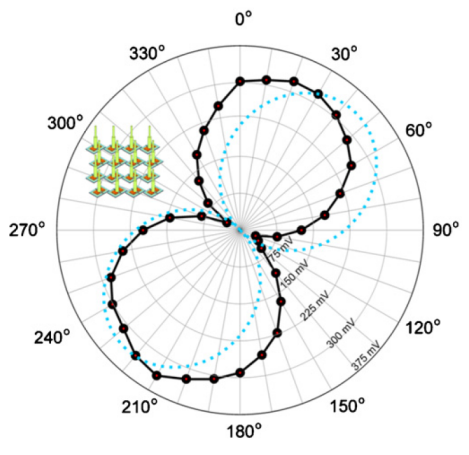

(a)

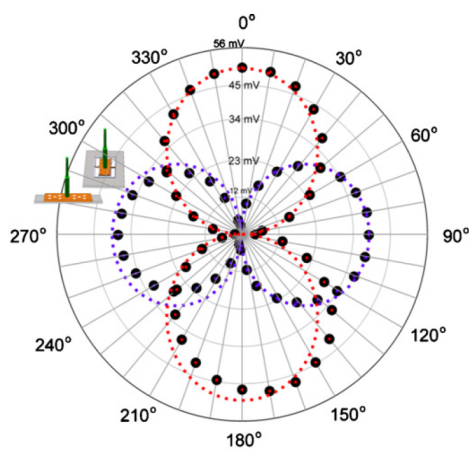

(b)

Figure 1.8: Directivity measurements for the (a) arrayed hair flow sensor and (b) single hair flow sensor. The measured directivity shapes (bullets) are compared with perfect figures-of-eight (dashed lines) oriented in the same direction as the sensor orientation on the die. The maximum sensitivity axis of the arrayed hair flow sensor was tilted $45^{\circ}$ relative to the long axis of the sensor die due to the sensor design while the single hair flow sensor is mounted with a $0^{\circ}$ angle (Dagamseh et al., 2013).

applicability and feasibility in our artificial hair flow sensory system. In this research, the following mechanisms are addressed.

Adaptation may be obtained using dc-biasing of the electrode structures to utilize effective stiffness weakening by electrostatic forces. The effective springstiffness can be reduced by increasing the dc-bias voltage and even be made negative. Hence, resonance frequency, sensitivity and quality factor of the hairs may be changed adaptively to accommodate optimal signal reception and provide frequency selectivity.

Tunable filtering of the artificial hair flow sensor is investigated by using additional ac-signals at frequencies chosen at will, since the capacitive structures are amenable to electrostatic actuation. Voltage controlled actuation implies non-linearity, since the electrostatic torque depends non-linearly on the voltage as well as on the angle, which allows for implementing a timedependent torsional stiffness. In addition to filtering, other applications of additional ac-signals to our hair flow sensory system will be discussed.

Stochastic resonance is a stochastic concept which comprises the use of available thermal and acoustic noise, or mechanical noise through electrostatic actuation. The mechanism of stochastic resonance is studied and demonstrated in a MEMS comb-drive based system. By controlling several system 
parameters electrostatically, the conditions for stochastic resonance are fully controllable.

\subsubsection{Hair flow sensors}

In addition to the research of mechanisms for enhancing the performance of (bioinspired) sensing systems, also other aspects of the biomimetic hair flow sensors are addressed and investigated.

Characterization of fabricated flow sensors is essential for determining the sensor's performance. Therefore, three different sources for generating harmonic airflows, each with its advantages and disadvantages regarding acoustic impedance, frequency range and maximum velocity, are investigated. We discuss the impact of cross-sensitivity when simultaneously sensing pressure and flow velocity and how to distinguish between these contributions in actual measurements.

Performance assessment of a bio-inspired hair flow sensor is of great importance for realizing a 'good' hair flow sensor. The hair mechanics of both the cricket and our flow sensor are thoroughly analysed, from which five independent metrics and a figure of merit (FoM) are derived. These metrics and the FoM are evaluated and compared for cricket and MEMS.

\subsubsection{Bio-inspired sensors}

Based on the fabrication process of the artificial hair flow sensors and the fabrication process of micro Coriolis mass flow sensors (Haneveld et al., 2010), three artificial bio-inspired inertial sensor systems are investigated.

A biomimetic accelerometer is inspired by the cricket's clavate hair (Murphey, 1981). These clavate hairs turn out to be sensitive to (gravitational) acceleration, providing the cricket information about its orientation. We discuss the design and performance of our biomimetic clavate hair system.

A biomimetic gyroscope is inspired by the fly's haltere structures (Pringle, 1948). Halteres are tiny club-shaped organs that beat anti-phase to the wings during flight, and function as gyroscopes by measuring the flies body rotation using Coriolis forces. The development of our haltere-inspired gyroscope is discussed.

An angular accelerometer , bio-inspired by the semicircular channel in the vestibular system (Fernández and Goldberg, 1971). The accelerometer consists of a 
water-filled tube, wherein the fluid flow velocity is measured thermally as a representative for the external angular acceleration. We discuss the design, development and performance of this angular accelerometer.

\subsection{Outline}

The organization of this thesis is as follows. Chapter 2 continues with three oscillatory flow setups and their impact on characterization of our hair flow sensors. Adaptation by applying a dc-bias voltage to the capacitive structures of the bio-inspired hair flow sensors is discussed in chapter 3. The impact of applying an ac-bias voltage on achieving selective gain and tunable filtering is treated in chapter 4 . Chapter 5 continues with the application of an ac-bias voltage to the capacitive structures, but here for the case where the flow frequency is chosen to be much lower than the voltage frequency. As a result, electro mechanical amplitude modulation can be achieved, which allows for improving the signal-to-noise ratio. Stochastic resonance (SR) in a voltage-controlled MEMS-slider is discussed in chapter 6 . By judiciously choosing the applied voltages on the slider's capacitive structures, it is demonstrated that the signal-to-noise ratio can be improved by adding noise.

In chapter 7 the development and characterization of a cricket-inspired hairbased accelerometer is described. Its mechanics are investigated and design rules are derived for maximizing the acceleration-induced response. The design and fabrication of a biomimetic gyroscope inspired by the fly's haltere is discussed in chapter 8 . The gyroscope dynamics for the fly's haltere are treated and design rules are derived for realization of a 'good' gyroscope. Measurements for the fabricated bio-inspired gyroscope are discussed and a comparison is made between the fly's haltere and the realized biomimetic gyroscope. The design, development and performance of the bio-inspired angular accelerometer is discussed in chapter 9 . The fluid dynamics and read-out principles are explained and first measurements are presented.

In chapter 10 the performance of hair flow sensors is assessed, based upon five metrics and a figure of merit. These criteria are numerically evaluated for both the cricket and our bio-inspired hair flow sensor, and the impact of the hair length for optimization is investigated. Chapter 11 summarizes the achievements of the research described in this thesis. Also, next steps and other possible directions for further research in the fields of this research are proposed. 


\section{References}

Alagirisamy, P., Jeronimidis, G., and Moal, V. L. (2009). An investigation of viscousmediated coupling of crickets cercal hair sensors using a scaled up model. In Proc. SPIE, vol. 7401.

Avitabile, D., Homer, M., Champneys, A. R., Jackson, J. C., and Robert, D. (2010). Mathematical modelling of the active hearing process in mosquitoes. J. R. Soc. Interface, 7:105-122.

Bathellier, B., .Barth, F., Albert, J., and Humphrey, J. (2005). Viscosity-mediated motion coupling between pairs of trichobothria on the leg of the spider Cupiennius salei. J. Comp. Physiol. A, 191:733746.

Bruinink, C. M., Jaganatharaja, R. K., de Boer, M. J., Berenschot, J. W., Kolster, M. L., Lammerink, T. S. J., Wiegerink, R. J., and Krijnen, G. J. M. (2009). Advancements in technology and design of biomimetic flow-sensor arrays. In Proc. MEMS 2009, pp. 152-155.

Casas, J., Steinmann, T., and Krijnen, G. (2010). Why do insects have such a high density of flow sensing hairs? Insights from the hydromechanics of biomimetic MEMS sensors. J. R. Soc. Interface, 7(51):1487-1495.

Chen, N., Tucker, C., Engel, J., Yang, Y., Pandya, S., and Liu, C. (2007). Design and characterization of artificial haircell sensor for flow sensing with ultrahigh velocity and angular sensitivity. J. Microelectromech. S., 16:999-1014.

Cummins, B., Gedeon, T., Klappera, I., and Cortez, R. (2007). Interaction between arthropod filiform hairs in a fluid environment. J. Theor. Biol., 247:266280.

Dagamseh, A. M. K., Bruinink, C. M., Droogendijk, H., Wiegerink, R. J., Lammerink, T. S. J., and Krijnen, G. J. M. (2010). Engineering of biomimetic hair-flow sensor arrays dedicated to high-resolution flow field measurements. In Proc. IEEE Sensors 2010, pp. 2251-2254, Waikoloa, HI, United States.

Dagamseh, A. M. K., Bruinink, C. M., Wiegerink, R. J., Lammerink, T. S. J., Droogendijk, H., and Krijnen, G. J. M. (2013). Interfacing of differentialcapacitive biomimetic hair flow-sensors for optimal sensitivity. J. Micromech. Microeng., 23:035010.

Dagamseh, A. M. K., Wiegerink, R. J., Lammerink, T. S. J., and Krijnen, G. J. M. (2012). Towards a high-resolution flow camera using artificial hair sensor arrays for flow pattern observations. Bioinsp. Biomim., 7:046009. 
Dangles, O., Magal, C., Pierre, D., Olivier, A., and Casas, J. (2005). Variation in morphology and performance of predator-sensing system in wild cricket populations. J. Exp. Biol., 208(3):461-468.

de Bree, H.-E. (2003). An overview of microflown technologies. Acta Acust. United Ac., 89:163-172.

Dickinson, B. (2010). Hair receptor sensitivity to changes in laminar boundary layer shape. Bioinsp. Biomim., 5:016002.

Dijkstra, M., van Baar, J. J. J., Wiegerink, R. J., Lammerink, T. S. J., de Boer, J. H., and Krijnen, G. J. M. (2005). Artificial sensory hairs based on the flow sensitive receptor hairs of crickets. J. Micromech. Microeng., 15:S132-S138.

Fan, Z., Chen, J., Zou, J., Bullen, D., Liu, C., and Delcomyn, F. (2002). Design and fabrication of artificial lateral line flow sensors. J. Micromech. Microeng., 12:655-661.

Fernández, C. and Goldberg, J. M. (1971). Physiology of peripheral neurons innervating semicircular canals of the squirrel monkey. II. Response to sinusoidal stimulation and dynamics of peripheral vestibular system. J. Neurophysiol., 34(4):661-675.

Haneveld, J., Lammerink, T. S. J., de Boer, M. J., Sanders, R. G. P., Mehendale, A., Lötters, J. C., Dijkstra, M., and Wiegerink, R. J. (2010). Modeling, design, fabrication and characterization of a micro Coriolis mass flow sensor. $J$. Micromech. Microeng., 20:125001.

Krijnen, G. J. M., Dijkstra, M., van Baar, J. J., Shankar, S. S., Kuipers, W. J., de Boer, R. J. H., Altpeter, D., Lammerink, T. S. J., and Wiegerink, R. J. (2006). MEMS based hair flow-sensors as model systems for acoustic perception studies. Nanotechnology, 17:S84-S89.

Levin, J. E. and Miller, J. P. (1996). Broadband neural encoding in the cricket cercal sensory system enhanced by stochastic resonance. Nature, 380:165-166.

Lewin, G. and Hallam, J. (2010). A computational fluid dynamics model of viscous coupling of hairs. J. Comp. Physiol. A, 196:385-395.

McConney, M. E., Chen, N., Lu, D., Hu, H. A., Coombs, S., Liu, C., and Tsukruk, V. V. (2009). Biologically inspired design of hydrogel-capped hair sensors for enhanced underwater flow detection. Soft Matter, 5:292295. 
Murphey, R. K. (1981). The structure and development of a somatotopic map in crickets: The cereal afferent projection. Dev. Biol., 88:236-246.

Ozaki, Y., Ohyama, T., Yasuda, T., and Shimoyama, I. (2000). An air flow sensor modeled on wind receptor hairs of insects. In Proc. MEMS 2000, pp. 531-536, Miyazaki, Japan.

Pringle, J. W. S. (1948). The gyroscopic mechanism of the halteres of diptera. Philos. Trans. R. Soc. London B., 233(602):347-384.

Sadeghi, M., Peterson, R., and Najafi, K. (2011). Micro-hydraulic structure for high performance biomimetic air flow sensor arrays. In Tech. Digest-IEDM, 6131638, pp. 29.4.1-29.4.4.

Schnitzler, H. U., Moss, C. F., and Denzinger, A. (2003). From spatial orientation to food acquisition in echolocating bats. Trends in Ecol. and Evol., 18:386-394.

Shimozawa, T., Kumagai, T., and Baba, Y. (1998). Structural scaling and functional design of the cercal wind-receptor hairs of cricket. J. Comp. Physiol. A, 183:171186.

Shimozawa, T., Murakami, J., and Kumagai, T. (2003). Cricket wind receptors: thermal noise for the highest sensitivity known. In Sensors and sensing in biology and engineering, pp. 145-159. Springer.

Tao, J. and Yu, X. (2012). Hair flow sensors: from bio-inspiration to biomimicking—a review. Smart Mater. Struct., 21:113001.

van Baar, J. J., Dijkstra, M., Wiegerink, R. J., Lammerink, T. S. J., and Krijnen, G. J. M. (2003). Fabrication of arrays of artificial hairs for complex flow pattern recognition. In Proc. IEEE Sensors 2003, pp. 332-336, Toronto, Canada.

Wang, Y.-H., Lee, C.-Y., and Chiang, C.-M. (2007). A MEMS-based air flow sensor with a free-standing microcantilever structure. Sensors, 7:2389-2401.

White, R. D. and Grosh, K. (2005). Microengineered hydromechanical cochlear model. PNAS, 102:1296-1301.

Yang, Y., Klein, A., Bleckmann, H., and Liu, C. (2011). Artificial lateral line canal for hydrodynamic detection. Appl. Phys. Lett., 99:023701. 


\section{2 \\ Characterization}

\subsection{Introduction}

To investigate, characterize and understand the response of these type of MEMSbased hair-sensors, three different techniques are exploited for oscillatory airflow measurements. Each of these principles has specific properties regarding frequency range, pressure field and bandwidth. The advantages and disadvantages of each oscillatory flow setup are investigated and an overview is given which can help select the most suitable flow source.

\subsection{Theory and modelling}

\subsubsection{Oscillating airflow}

In case of a uni-directional oscillatory airflow, the velocity $v(t)$ can be described as:

$$
v(t)=v_{0} e^{j \omega t}
$$

This chapter is based on "Characterization of bio-inspired hair flow sensors: techniques to measure the response for both flow and pressure" by H. Droogendijk, A. M. K. Dagamseh, D. R. Yntema, R. G. P. Sanders, and G. J. M. Krijnen, submitted to Meas. Sci. Technol., (2014). 


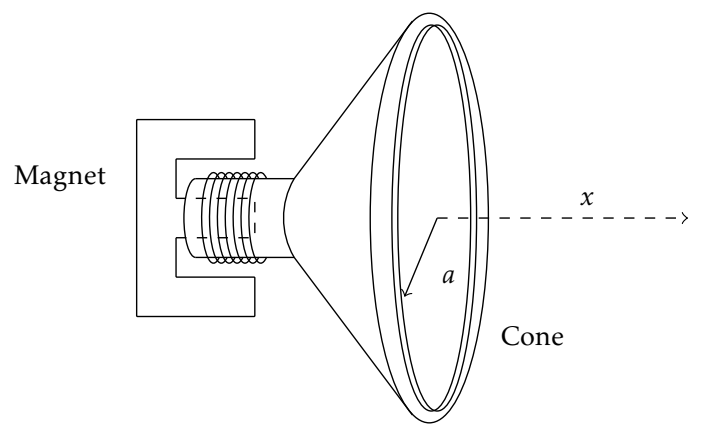

Figure 2.1: Schematic view of a loudspeaker

where $v_{0}$ is the velocity amplitude and $\omega$ is the angular frequency ${ }^{1}$. Under the assumption of incompressible airflow, the oscillatory flow can be defined as bulk flow. However, when the fluid is compressible, the flow can be described as sound and the harmonic velocity of the medium is called particle velocity. Consequently, there are two velocities present in the system; the propagation velocity of the sound wave $c$ and the local excursions of molecules when displaced by the pressure differences of the sound wave, called particle velocity $v$. In air, the propagation (phase) velocity is often referred to as the speed of sound.

\subsubsection{Loudspeaker}

A frequently used source for generation of oscillatory flows is a loudspeaker. There are many types of loudspeakers available, each with their own characteristics (i.e. cone radius, resonance frequency). In this work, we consider a loudspeaker with a flat cone with a radius $a$ of about $5 \mathrm{~cm}$, as illustrated in figure 2.1. In our case, the hair flow sensor is positioned close to the centre of the cone and the cone size is considerably smaller than the wavelength of sound $\lambda$. Although there is no clear baffle present, we can treat the loudspeaker for analysis as a baffled, plane circular piston source with radius $a$ and cone velocity amplitude $v_{0}$ for describing the velocity and pressure characteristics within tolerable accuracies (Crane, 1967). Consequently, the axial velocity potential $\Phi(x, t)$ is given by (Beissner, 1982):

$$
\Phi(x, t)=\frac{v_{0}}{j k} e^{j(\omega t-k x)}\left(1-e^{-2 j \gamma}\right),
$$

where $k$ is the wave number $(k=\omega / c)$ and $\gamma$ is a dimensionless parameter:

\footnotetext{
${ }^{1}$ In this work, we use complex notation and take the observable quantities implicitly to be the respective real parts.
} 


$$
\gamma=\frac{k}{2}\left(\sqrt{a^{2}+x^{2}}-x\right)
$$

Differentiating with respect to space, the particle velocity profile $v_{x}$ along the axial distance $x$ can be calculated according to (Lamb, 1932):

$$
v_{x}(x)=-\frac{\partial \Phi(x, t)}{\partial x}=v_{0} e^{j(\omega t-\kappa x)}\left(1-\eta e^{-2 j \gamma}\right),
$$

where the dimensionless parameter $\eta$ is defined as:

$$
\eta=\frac{x}{\sqrt{a^{2}+x^{2}}}
$$

Similarly, the pressure dependence $p(x)$ along the axial distance is found to be as:

$$
p(x)=\rho \frac{\partial \Phi(x, t)}{\partial t}=\rho c v_{0} e^{j(\omega t-\kappa x)}\left(1-e^{-2 j \gamma}\right),
$$

where $\rho$ is the density of the medium and $c$ is the speed of sound in the medium. The acoustic impedance $Z_{x}(x)$ in axial direction $x$ is defined as the ratio of pressure $p(x)$ and magnitude of the particle velocity $v_{x}(x)$ :

$$
Z_{x}(x)=\frac{p(x)}{v_{x}(x)}=\rho c\left(\frac{1-e^{-2 j \gamma}}{1-\eta e^{-2 j \gamma}}\right) .
$$

Using these expressions, the loudspeaker characteristics can be divided into three regions: the far field, near field and very near field. In the far field region, the speaker is acting as a radiating source, where both particle velocity and pressure are decreasing with $r^{-1}$. In the near field region the pressure decreases with $r^{-1}$, but particle velocity with $r^{-2}$. Since in the very near field the term $k r \ll 1$ the difference between compressible and incompressible airflow vanishes; the particle velocity matches the cone velocity, whereas the pressure amplitude is constant (de Bree et al., 2004). A schematic overview of the various regions is given in figure 2.2.

In our case, the very near field region lies within $1 \mathrm{~cm}$ distance away from the loudspeaker source, which is defined by $r<a /(2 \pi)$, where $r$ is the distance to the cone and $a$ is the radius of the cone. Operation in the near field occurs in the range from $1-10 \mathrm{~cm}$. Larger distances satisfying $r>c /(2 \pi f)$ lead to far field behaviour, where $c$ is the speed of sound and $f$ is the flow frequency. The decrease of particle velocity along the axial distance is measured using a Microflown sensor (de Bree, 2003) and compared to theory in figure 2.2.

The resonance frequency of the loudspeaker that will be used (Visaton WS 17 E) lies around $35 \mathrm{~Hz}$, making the loudspeaker suitable for flow frequencies ranging 


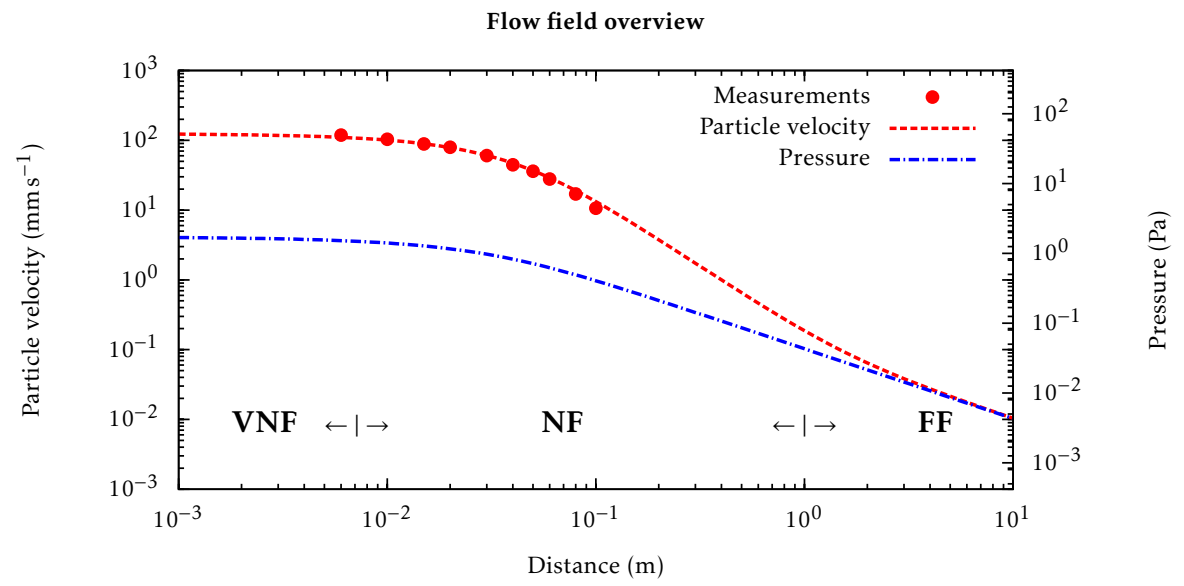

Figure 2.2: Measurement of decreasing particle velocity as function of the distance to the cone at a flow frequency of $100 \mathrm{~Hz}$. Also, the calculated pressure and particle velocity versus distance to the cone are shown, divided in the three regions: very near field (VNF), near field (NF) and far field (FF).

from $10-1000 \mathrm{~Hz}$. For higher frequencies the cone is not moving uni-directionally anymore, as observed using a Polytec Laser Scanning Vibrometer (Polytec, 2005), making it difficult to use this actuator for oscillating flow at frequencies $>1 \mathrm{kHz}$. Additionally, beyond resonance the required voltage amplitude for a given cone velocity increases with $10 \mathrm{~dB}$ decade $^{-1}$.

\subsubsection{Vibrating sphere}

Another type of flow source used is the vibrating sphere, which can be considered as a dipole source. To compare for the near field condition, in which the hair flow sensor is positioned close to the source, the flow field decays with $r^{-2}$ for the loudspeaker, but with $r^{-3}$ for the dipole, where $r$ is the distance to the source (Milne-Thompson, 1955). A schematic view of the flow field generated around the sphere is shown in figure 2.3, where $x$ is the direction in which the sphere vibrates and $D$ is the distance between the axial direction $x$ and the sensor.

In literature, the dipole source (represented by a vibrating sphere) is commonly used as a hydrodynamic stimulus in lateral-line system related studies since it imitates the movement of aquatic animals and thus can be used as dummy prey or predator under certain assumptions (Coombs et al., 1996; Harris and van Bergeijk, 1962). Bio-physicists have used the dipole field theory to estimate the prey 


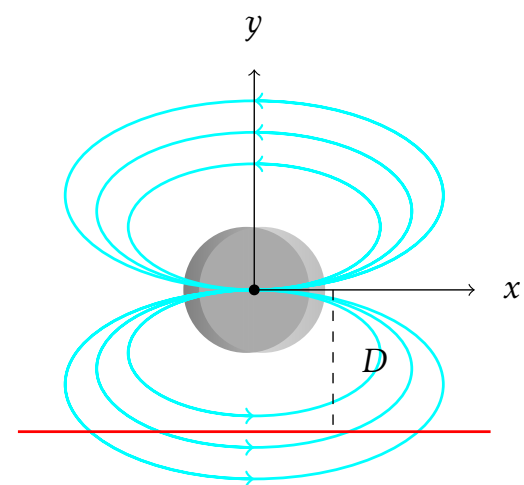

Figure 2.3: Sketch of a dipole velocity field.

localization methodology in fish (Goulet et al., 2008). This methodology is based on determining the characteristic points of the dipole field. These theoretical models investigate and describe the interactions between the lateral-line neuromast and surrounding fluid by applying and solving the Navier-Stokes equation (Acheson, 1990; Franosch et al., 2005):

$$
\frac{\partial \vec{V}}{\partial t}+(\vec{V} \cdot \nabla) \vec{V}=-\frac{\nabla P}{\rho}+v \nabla \vec{V}+\vec{g}
$$

where $\vec{V}$ is the fluid velocity, $P$ the pressure, $\rho$ the fluid density, $\vec{g}$ the acceleration of gravity and $v$ the kinematic viscosity. Following Lamb (1932), the dipole velocity flow field can be described by the velocity potential $\Phi$ with sphere radius $a$ and harmonic sphere velocity amplitude $v_{0}$ :

$$
\Phi(r, \theta, t)=\frac{a^{3}}{2 r^{2}}(1+j k r) \cos (\theta) v_{0} e^{j(\omega t-k r)},
$$

where $r$ is the radial distance and $\theta$ is the angle with respect to the axial direction. With the velocity as the negative gradient of the velocity potential $(-\nabla \Phi)$, the flow velocity at each position can be predicted. For the assumption of having incompressible flow close to the source $(k r \ll 1)$ and the fact that $r=\sqrt{x^{2}+y^{2}}$, the velocity potential simplifies to:

$$
\Phi(x, y, t)=\frac{a^{3}}{2} \frac{x}{\left(x^{2}+y^{2}\right)^{3 / 2}} v_{0} e^{j \omega t} .
$$

Taking into account and that the hair flow sensor is positioned at distance $y=D$ from the $x$-axis and that the sphere vibrates along the $x$-axis, the flow velocity 
projections in $x$ and $y$ directions are consequently given by:

$$
v_{x}(x, t)=v_{0} e^{j \omega t} \frac{a^{3}}{2} \frac{2 x^{2}-D^{2}}{\left(x^{2}+D^{2}\right)^{5 / 2}}, \quad v_{y}(x, t)=v_{0} e^{j \omega t} \frac{a^{3}}{2} \frac{3 D x}{\left(x^{2}+D^{2}\right)^{5 / 2}},
$$

where $v_{x}(x)$ is the $x$-component of the velocity field and $v_{y}(x)$ the $y$-component. Notice that these expressions are valid in the near field (i.e. close to the sphere) only, since the flow is assumed there to be incompressible. At larger distances, the source becomes a radiating dipole source generating the usual far-field waves, for which the flow needs to be considered as compressible. Using the velocity potential from $(2.10)$, the pressure dependency $p(x, t)$ along the axial distance is found to be as:

$$
p(x, t)=\rho \frac{\partial \Phi(x, y, t)}{\partial t}=j \rho \omega v_{0} e^{j \omega t} \frac{a^{3}}{2} \frac{x}{\left(x^{2}+D^{2}\right)^{3 / 2}} .
$$

Subsequently, the acoustic impedance $Z_{x}(x)$ in the axial direction $x$ can then be calculated as:

$$
Z_{x}(x)=\frac{p(x)}{v_{x}(x)}=j \rho c k x\left(\frac{1}{2}+\frac{3}{2}\left[\frac{D^{2}}{2 x^{2}-D^{2}}\right]\right),
$$

where $k$ is the wave number $(k=\omega / c)$. This expression shows that the acoustic impedance increases with flow frequency $\omega$ and is theoretically zero when performing measurements just below the vibrating sphere $(x=0)$. Similarly, the near-field acoustic impedance $Z_{y}(x)$ for flow in $y$-direction can be calculated as:

$$
Z_{y}(x)=\frac{p(x)}{v_{y}(x)}=j \rho c k\left(\frac{x^{2}+D^{2}}{3 D}\right) .
$$

\subsubsection{Standing wave tube}

A well defined velocity profile of a compressible oscillating flow, i.e. particle velocity, is found inside a standing wave tube (de Bree et al., 1999). Such a tube is given in figure 2.4, wherein we have a closed tube with length $L$, a loudspeaker on the left side and a reference pressure sensor on the right side. The sensor for characterization is placed inside at a distance $x$ from the loudspeaker. For frequencies lower than a so-called cut-off frequency $f_{c}$, the sound wave inside the tube can be approximated by a plain wave. Above this frequency, standing waves can also occur perpendicular to the $x$-direction. The cut-off frequency for a cylindrical tube is given by Dowling and Williams (1983): 


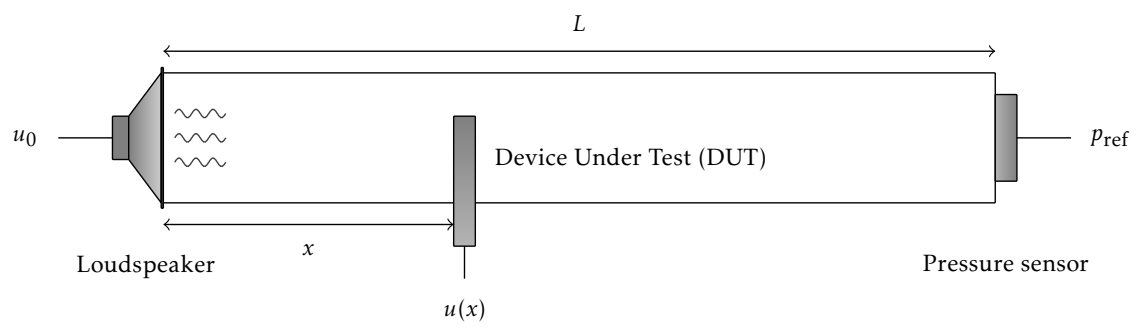

Figure 2.4: Overview of a standing wave tube.

$$
f_{\mathrm{c}}=\frac{c}{1.71 d}
$$

For a tube with a diameter $d=5 \mathrm{~cm}$ operating in air, the cut-off frequency $f_{\mathrm{c}}$ is about $4 \mathrm{kHz}$. Below the cut-off frequency the plane wave inside the tube can be described using complex sound pressure:

$$
p(x, t)=p_{0}\left(A e^{-j k x}+B e^{j k x}\right) e^{j \omega t},
$$

where $A$ and $B$ are complex numbers. Using conservation of momentum:

$$
\rho \frac{\partial v_{x}}{\partial t}=-\frac{\partial p}{\partial x}
$$

Since $c=\omega / k$, the associated particle velocity $v_{x}$ in the axial direction $x$ can be calculated:

$$
v_{x}(x, t)=\frac{p_{0}}{\rho c}\left(A e^{-j k x}-B e^{j k x}\right) e^{j \omega t} .
$$

The constants $A$ and $B$ can be calculated by the boundary conditions $\left(v_{x}(L)=0\right.$ and $\left.v_{x}(0)=v_{0}\right)$, where $v_{0}$ is the cone velocity of the loudspeaker:

$$
A e^{-j k L}=B e^{j k L}, \quad A-B=\frac{v_{0} \rho c}{p_{0}} .
$$

Thus, the particle velocity amplitude $v_{x}(x)$ in the axial direction $x$ can be calculated on every position $x$ inside the tube according to:

$$
v_{x}(x)=v_{0} \frac{\sin (k(L-x))}{\sin (k L)},
$$

and the pressure inside the tube can be represented as: 


$$
p(x)=-j \rho c v_{0} \frac{\cos (k(L-x))}{\sin (k L)} .
$$

The resulting acoustic impedance $Z_{x}(x)$ in the axial direction $x$, the ratio between the pressure $p(x)$ and the particle velocity $v_{x}(x)$, is:

$$
Z_{x}(x)=\frac{p(x)}{v_{x}(x)}=-j \frac{\rho c}{\tan (k(L-x))} .
$$

Thus, the particle velocity $v_{x}$ at the position of the sensor $x_{0}$ can be calculated as:

$$
v_{x}=\frac{j}{\rho c} p_{\text {ref }} \sin \left(k\left(L-x_{0}\right)\right),
$$

where $p_{\text {ref }}$ is the pressure from the pressure reference sensor. Since all parameters are known, or can be easily determined by measurement, it is possible to determine the response of the sensor for a given particle velocity $u$ and angular frequency $\omega$. A useful property of the standing wave tube is that the pressure at position $x_{0}$ can be represented as:

$$
p=p_{\text {ref }} \cos \left(k\left(L-x_{0}\right)\right) .
$$

An incidental advantage when using a standing wave tube is that the sound stays 'inside' the tube, allowing for high sound intensities at high frequencies without the risk of hearing damage. Therefore, the SWT is a very suitable flow source for high flow frequencies.

\subsection{Bio-inspired hair flow sensors}

The hair flow sensors, which will be characterized using the described oscillatory flow setups, are described in chapter 1 . With the hair flow sensors designed and fabricated, their performance needs to be determined. In succession, three different types of oscillatory airflow setups are used to obtain information about the flow and pressure response of the cricket-inspired hair flow sensor. For this type of hair flow sensors, the developed airflow moves over a surface that can be considered flat. As a result, the velocity profile $v(y, t)$ can be described by (Panton, 1996; Steinmann et al., 2006):

$$
v(y, t)=v_{0} e^{j \omega t}\left(1-e^{-\beta y} e^{-j \beta y}\right),
$$

where $y$ is the distance to the surface and $\beta$ is proportional to the reciprocal of the boundary layer thickness $\delta_{b}$, with $v$ the kinematic viscosity: 


$$
\beta=\sqrt{\frac{\omega}{2 v}} .
$$

The hair length of our flow sensors is $800 \mu \mathrm{m}$, which is typically in the same order of the boundary layer thickness $\delta_{b}$. Further, the plate length of our flow sensor is about $200 \mu \mathrm{m}$, which is significantly smaller than the wavelength $\lambda$ of sound frequencies in the audible range. Therefore, the pressure drop over the sensor can be considered negligible and the hair rotates only due to flow-induced forces.

\subsubsection{Loudspeaker}

Using the loudspeaker as a flow source for characterisation of the MEMS hair flow sensor, its frequency transfer was determined optically using laser Doppler vibrometry (Polytec MSA 400) by measurement of the membrane displacement (Polytec, 2005). By first determining the electro mechanical transfer of the loudspeaker (see appendix B) and using the expressions for the near field both magnitude and phase of the sensor could be determined and are shown in figure 2.5. The experimental data is shown together with an analytical model for the hair flow sensor (Droogendijk et al., 2012).

\subsubsection{Vibrating sphere}

Figure 2.6 shows the dipole velocity field (parallel $v_{x}(x)$ and perpendicular $v_{y}(x)$ field components) for both model and measurements using our MEMS hair flow sensory system. The velocity field uniquely encodes the distance to the source irrespective of the fluid properties, vibration frequency, amplitude, direction and dimensions of the moving object (Dagamseh et al., 2010).

\subsubsection{Standing wave tube}

Figure 2.7 shows the acoustic properties of the standing wave tube as represented by the particle velocity $v_{x}$ and pressure $p$ plotted versus the frequency of the oscillating airflow. Furthermore, measurements performed capacitively using the MEMS hair flow sensor are included in the figure as well. The plots in figure 2.7 facilitate determining whether the sensor is sensitive to particle velocity, pressure or a combination of both using the peaks and the dips. We observe that the considered hair flow sensor mainly shows a response to pressure. The outcome of this experiment was verified by rotating the sensor over different angles $\left(-90^{\circ}, 0^{\circ}\right.$ and $90^{\circ}$ ), for which all experiments showed similar results. 

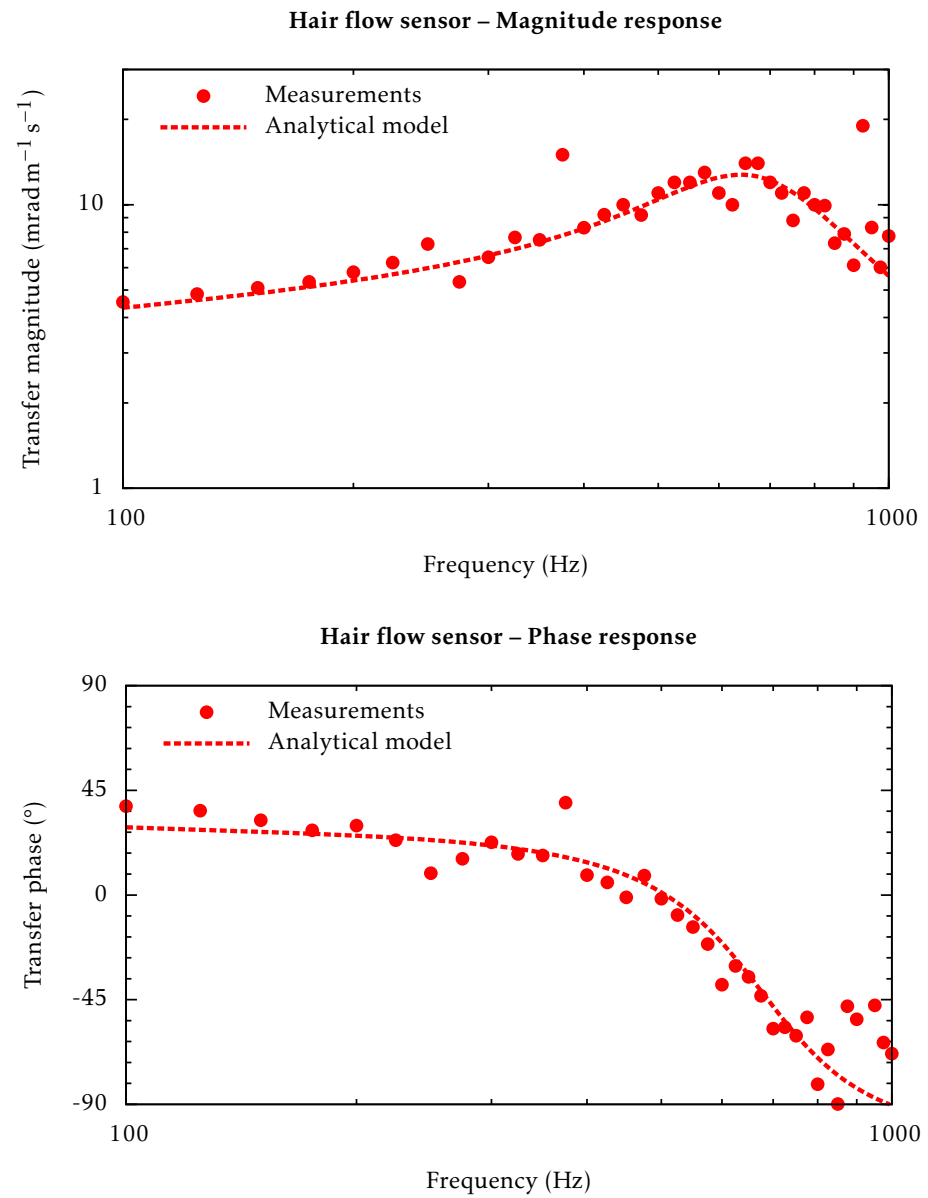

Figure 2.5: Magnitude and phase response of the hair flow sensor using a loudspeaker as flow source.

\subsection{Discussion}

Various properties have been investigated using three different types of oscillatory flow setups. From both the loudspeaker and vibrating sphere setup it was found that the hair flow sensor mainly shows a response to particle velocity, whilst using the standing wave tube the response was observed to be mainly due to pressure. To explain these experimental outcomes, another measurement was performed for the loudspeaker setup by capacitively interrogating the hair flow sensor instead of using laser Doppler vibrometry. As a result, now both the flow response (in 


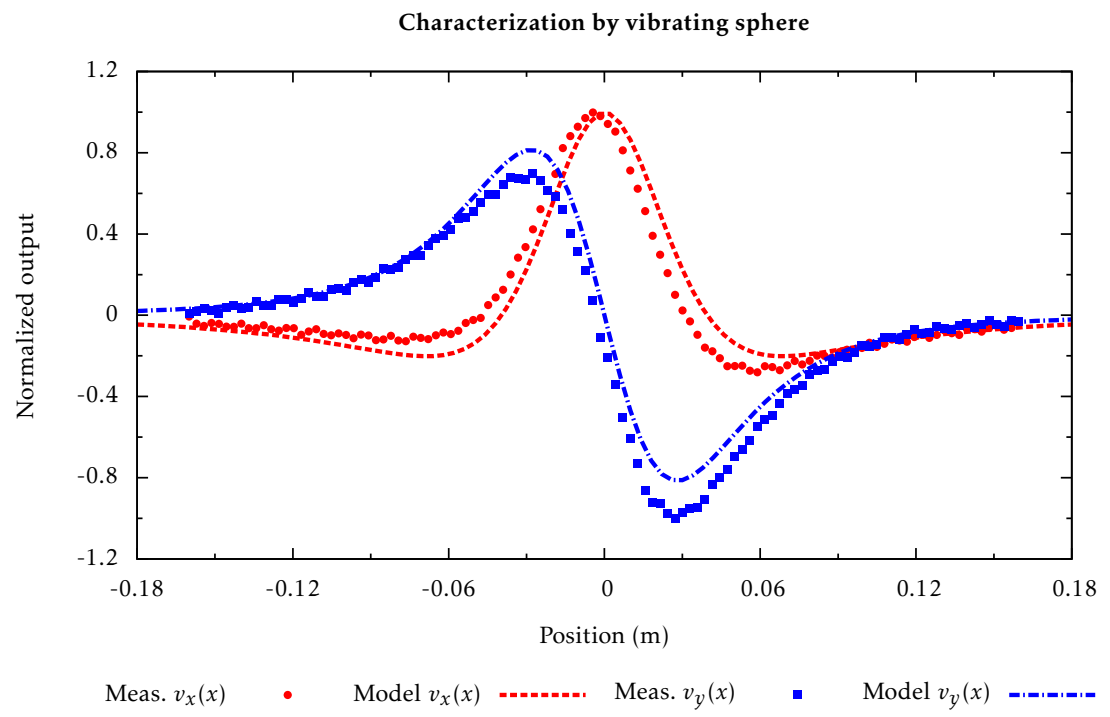

Figure 2.6: Both model and measurement for the parallel component $v_{x}(x)$ and the perpendicular component $v_{y}(x)$ of the flow field at a frequency of $20 \mathrm{~Hz}$ for a vibrating sphere (Dagamseh et al., 2010).

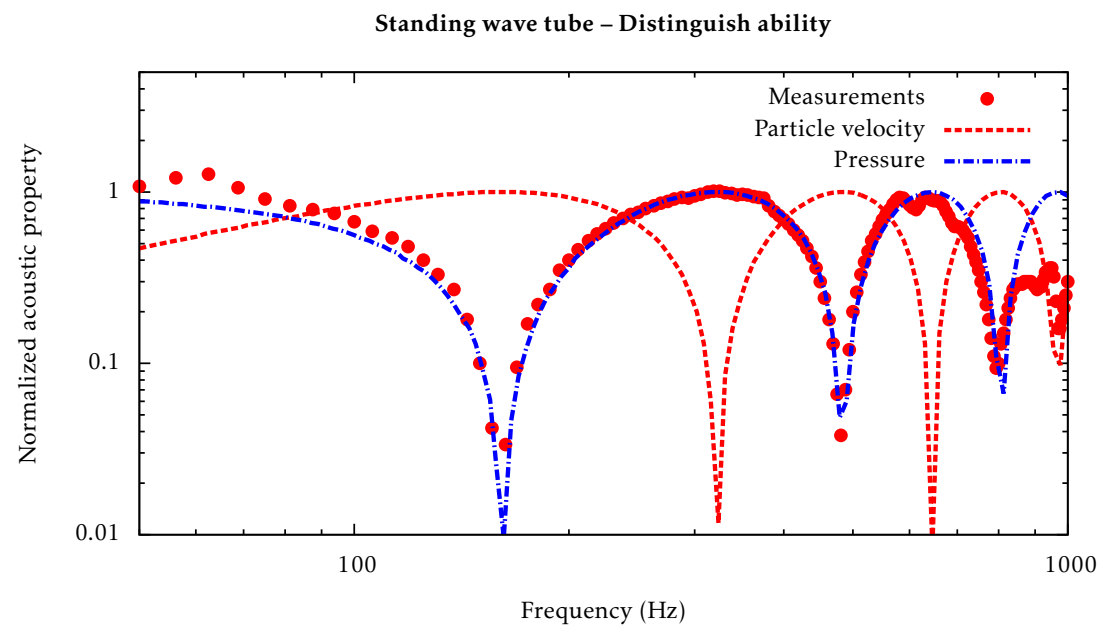

Figure 2.7: Normalized acoustic properties of the SWT for both pressure and particle velocity. Also, the measured response of the biomimetic hair flow sensor is shown.

case of the LS) and the pressure response (in case of the SWT) are determined by capacitive read-out. The obtained acoustic property responses are shown in 


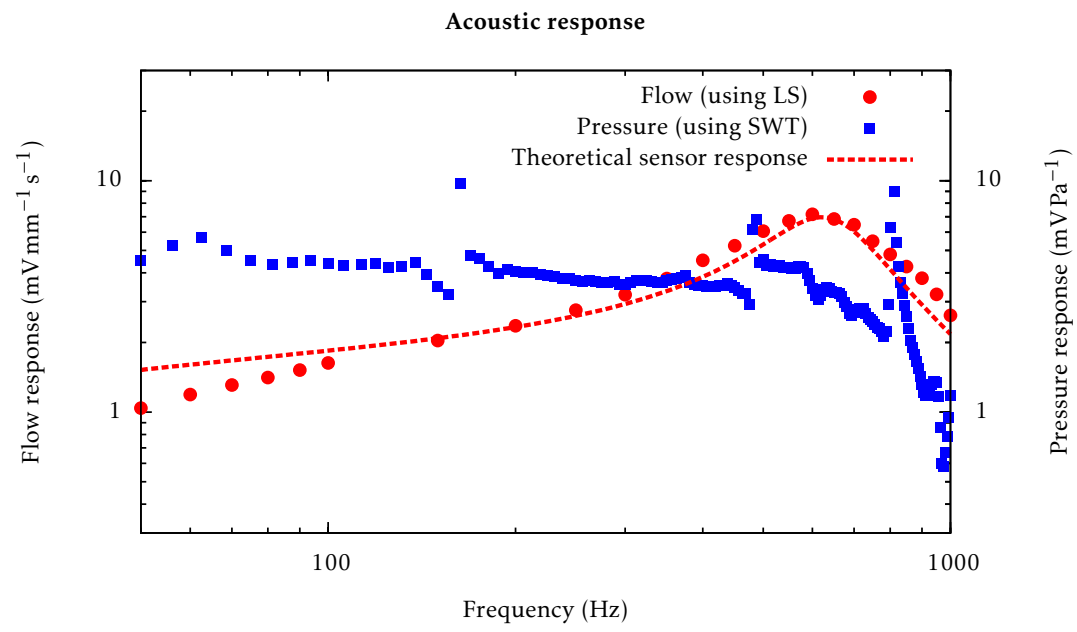

Figure 2.8: Determination of the response to both flow (using LS) and pressure (using SWT) of the MEMS hair flow sensor, compared to the analytical model giving the theoretical sensor response. Both responses are corrected for the transfer by the oscillatory flow source.

figure 2.8, together with the theoretical response based upon the analytical model from (Droogendijk et al., 2012).

Although it is clear that the analytical model of the biomimetic hair flow sensor is in agreement with the flow measurements performed using the loudspeaker, the observed pressure response of the sensor using the SWT was not predicted. To explain this pressure sensitivity of the sensor, two aspects need to be addressed. First, the mechanical reason for exhibiting a pressure response. Second, the fact that the SWT indicates a pressure sensitive system, although the LS shows a clear flow response.

The main mode of operation of the hair flow sensor is torsional-based movement of the hair and membrane by small hair rotations due to the incoming airflow. However, by considering the beam-suspension of the membrane, the sensor is also able to exhibit vertical movement of the membrane due to a limited vertical stiffness. Furthermore, the hair flow sensor shows a flow response which is almost critically damped (figure 2.5), indicating the significance of the squeeze film damping in the gap between the capacitor plates (Bao and Yang, 2007). Therefore, changes in pressure on the top side of the membrane lead to pressure differences over the membrane, inducing a pressure-dependent force acting on the membrane. As a consequence, due to the limited vertical stiffness, the capacitance will be affected by the acoustic pressure. Although read-out is 


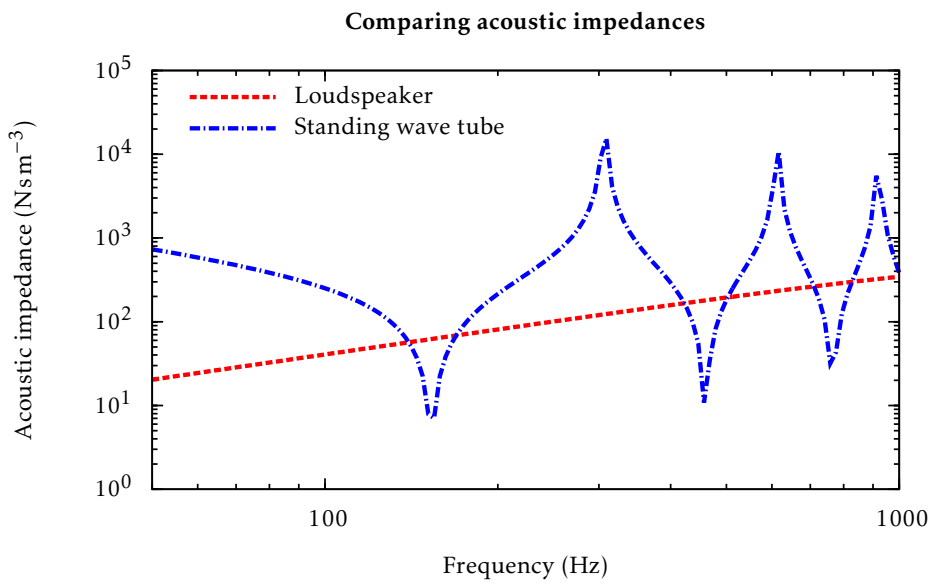

Figure 2.9: Acoustic impedance versus flow frequency for the loudspeaker and standing wave tube.

performed differentially and theoretically only a flow-induced response should be measurable, non-idealities in both the sensory system (e.g. misalignment and built-in charges (Wibbeler et al., 1998)) and read-out electronics (e.g. symmetry and biasing) can cause the pressure-response to become observable in the output voltage $U(v, p, \omega)$ due to the fact that the rotation angle may consequently consist of a small constant tilt $\theta_{0}$ (see appendix $\mathrm{C}$ for a derivation):

$$
U(v, p, \omega) \propto v H_{\mathrm{v}}(\omega)-p H_{\mathrm{p}}(\omega) \frac{2 \theta_{0}}{g},
$$

where $H_{\mathrm{v}}(\omega)$ and $H_{\mathrm{p}}(\omega)$ are the frequency-dependent mechanical transfer functions for flow and pressure respectively, and $g$ is the distance between the parallel plates of the capacitive structure.

To explain why the pressure dependence is only observed when using the SWT, the acoustic impedance $Z_{x}$ is considered. This impedance, defined as the ratio between the pressure $p$ and the particle velocity $v_{x}$ at specific position $x$, can be used to investigate whether the sensor is mainly sensitive to pressure, velocity or both. The vibrating sphere is used under very near field conditions $(k r \ll 1$, comparable to incompressible flow) with a well defined velocity profile around the sphere and negligible pressure difference. Thus, its acoustic impedance $Z_{x}$ is very low. A comparison of the acoustic impedance $Z_{x}$ for both the loudspeaker and standing wave tube is shown in figure 2.9.

According to the plots, the acoustic impedance of the standing wave tube is 
Table 2.1: Properties of oscillatory flow setups. For the loudspeaker and vibrating sphere, it is assumed that the flow sensor is placed close to the source.

\begin{tabular}{lccc}
\hline Property & Loudspeaker & Vibrating sphere & Standing wave tube \\
\hline Frequency range & $10-1000 \mathrm{~Hz}$ & $10-100 \mathrm{~Hz}$ & $10-4000 \mathrm{~Hz}$ \\
Acoustic impedance & Low & Very low & Variable \\
Maximum velocity & up to $1 \mathrm{~m} \mathrm{~s}^{-1}$ & up to $1 \mathrm{~m} \mathrm{~s}^{-1}$ & up to $1 \mathrm{~m} \mathrm{~s}^{-1}$ \\
Distinguish ability & No & No & Yes \\
Sound intensity (ears) & High & Low & Low \\
\hline
\end{tabular}

generally higher than in case of the loudspeaker, except at the positions where the antinodes of the particle velocity occur. As an example, by considering a specific amplitude of the particle velocity $v_{x}$, the associated pressure fluctuations $p$ are much larger when using an SWT than compared to a loudspeaker. Then, it is possible that both the loudspeaker and SWT setups show a flow and pressure response. However, in case of the low $Z_{x}$ for the loudspeaker the output will be dominated by flow, whilst due to the high $Z_{x}$ for the SWT the output will be dominated by pressure.

To make a clear comparison between the oscillatory flow sources, an overview of several properties is given in table 2.1. For operation in the near field, a loudspeaker proves to be a suitable flow source for applying a particle velocity with a relatively low pressure within a large frequency bandwidth. A vibrating sphere becomes interesting for very small acoustic impedance measurements and measuring bulk flow. Benefits from a SWT setup are its ability to distinguish between particle velocity and pressure, and the practical circumstance that the sound stays inside the tube, allowing high sound intensities at high frequencies.

\subsection{Conclusion}

For extensive characterization of bio-inspired hair flow sensors, three different types of oscillatory flow setups are investigated and discussed. A loudspeaker, a vibrating sphere and a standing wave tube all have their own characteristics and depending on the application the appropriate source needs to be chosen. It is shown that combining insights from all three setups, more information is obtained about the sensor response then when solely using a specific source. 


\section{References}

Acheson, D. (1990). Elementary fluid dynamics. Oxford university press, Oxford.

Bao, M. and Yang, H. (2007). Squeeze film air damping in MEMS. Sens. Act. A: Phys., 136:3-27.

Beissner, K. (1982). On the plane-wave approximation of acoustic intensity. $J$. Acoust. Soc. Am., 71(6):1406-1411.

Coombs, S., Hastings, M., and Finneran, J. (1996). Modeling and measuring lateral line excitation patterns to changing dipole source locations. J. Comp. Physiol. A, 178:359-371.

Crane, P. H. G. (1967). Method for the calculation of the acoustic radiation impedance of unbaffled and partially baffled piston sources. J. Sound Vib., 5(2):257-277.

Dagamseh, A. M. K., Lammerink, T. S. J., Wiegerink, R. ., and Krijnen, G. J. M. (2010). Dipole-source localization using biomimetic flow-sensor arrays positioned as lateral-line system. Sens. Act. A: Phys., 162:355-360.

de Bree, H.-E. (2003). An overview of microflown technologies. Acta Acust. United Ac., 89:163-172.

de Bree, H.-E., Druyvesteyn, W. F., and Elwenspoek, M. C. (1999). Realisation and calibration of a novel half-inch p-u sound intensity probe. In Proc. $106^{\text {th }}$ AES, Munich, Germany.

de Bree, H.-E., Svetovoy, V. B., Raangs, R., and Visser, R. (2004). The very near field. Theory, simulations and measurements of sound pressure and particle velocity in the Very Near Field. In Proc. $11^{\text {th }}$ Int. Congress on Sound and Vibration, St. Petersburg, Russia.

Dowling, A. P. and Williams, J. E. (1983). Sound and sources of sound. Ellis Horwood.

Droogendijk, H., Bruinink, C. M., Sanders, R. G. P., Dagamseh, A. M. K., Wiegerink, R. J., and Krijnen, G. J. M. (2012). Improving the performance of biomimetic hair-flow sensors by electrostatic spring softening. J. Micromech. Microeng., 22(6):065026.

Franosch, J., Sichert, A., Suttner, M., and Hemmen, J. V. (2005). Estimating position and velocity of a submerged moving object by the clawed frog Xenopus and by fish - A cybernetic approach. Biol. Cybernetics, 93:231-238. 
Goulet, J., Engelmann, J., Chagnaud, B. P., Franosch, J.-M. P., Suttner, M. D., and van Hemmen, J. L. (2008). Object localization through the lateral line system of fish: theory and experiment. J. Comp. Physiol. A, 194:1-17.

Harris, G. G. and van Bergeijk, W. A. (1962). Evidence that the lateral-line organ responds to near-field displacements of sound sources in water. J. Acoust. Soc. Am., 34(34):1831-1841.

Lamb, H. (1932). Hydrodynamics. Cambridge University Press, Cambridge, $6^{\text {th }}$ edition.

Milne-Thompson, L. M. (1955). Theoretical hydrodynamics. The Macmillian Company, $3^{\text {rd }}$ edition.

Panton, R. L. (1996). Incompressible flow. Wiley.

Polytec (2005). MSA-400 Micro System Analyzer. Polytec, Waldbronn, Germany.

Steinmann, T., Casas, J., Krijnen, G., and Dangles, O. (2006). Air-flow sensitive hairs: boundary layers in oscillatory flows around arthropod appendages. J. Exp. Biol., 209:4398-4408.

Wibbeler, J., Pfeifer, G., and Hietschold, M. (1998). Parasitic charging of dielectric surfaces in capacitive microelectromechanical systems (MEMS). Sens. Act. A: Phys., 71:74-80. 


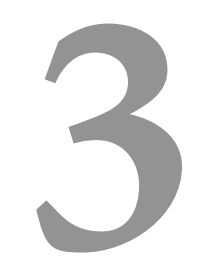

\section{Adaptation by tunable stiffness}

\subsection{Introduction}

Inspired by biology, the performance of a sensory system can be adapted to the environment. For example, the frog's internal ear displays adaptation by mechanical relaxation of hair bundles (Howard and Hudspeth, 1987), leading to a change in stiffness of the bullfrog's saccular hair cell. A similar technique can be applied to the biomimetic hair flow sensors by electrostatically reducing the torsional stiffness, by exploiting electrostatic spring softening (ESS) to increase the sensitivity and enhance the mechanical response of these sensors (Krijnen et al., 2006).

Previously, the possibility of ESS for our artificial hair flow sensors by dcbias voltages was demonstrated using electrostatic actuation (Floris et al., 2007). Here, we show that ESS can be used to adaptively change the mechanical transfer function of the system. In contrast to (Floris et al., 2007), we drive our flow sensors by airflow rather than actuating them electrostatically. We introduce the necessary models to fully describe ESS in our flow sensors. Additionally, we extend the work by demonstrating the use of ac-bias voltage based ESS and show its applicability

This chapter is based on "Improving the performance of biomimetic hair-flow sensors by electrostatic spring softening" by H. Droogendijk, C. M. Bruinink, R. G. P. Sanders, and G. J. M. Krijnen, published in J. Micromech. Microeng., 22(6): 065026, (2012), and "Advantages of electrostatic spring hardening in biomimetic hair flow sensors” by H. Droogendijk, M. J. de Boer, R. G. P. Sanders, and G. J. M. Krijnen, submitted to New J. Phys., (2014). 


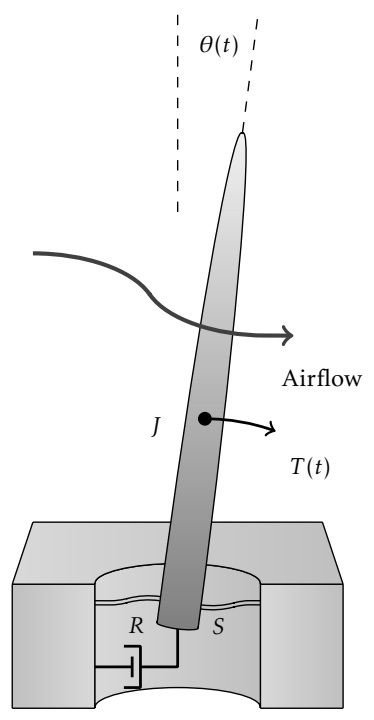

Figure 3.1: Model of a flow sensing hair based on an inverted pendulum (Shimozawa et al., 1998).

for capacitively interrogated sensors. By extending our artificial hair flow sensor with interdigitated comb finger structures, the system's torsional stiffness can be also increased electrostatically. Similar to ESS, the mechanism of electrostatic spring hardening (ESH) has consequences for the system's responsivity, bandwidth, threshold and (thermal) noise level.

\subsection{Electrostatic spring softening}

The motion of a flow susceptible hair is described by a second order mechanical system (figure 3.1) (Shimozawa et al., 1998), wherein a harmonic airflow causes the hair to periodically rotate due to a drag torque $T(t)$ caused by viscous forces (Stokes, 1851). The system's response is governed by its moment of inertia $J$, torsional resistance $R$ and torsional stiffness $S$ :

$$
J \frac{\mathrm{d}^{2} \theta(t)}{\mathrm{d} t^{2}}+R \frac{\mathrm{d} \theta(t)}{\mathrm{d} t}+S \theta(t)=T_{0} \cos (\omega t) .
$$

In our MEMS hair-flow sensory system, the torsional stiffness $S$ is controlled using a bias voltage on the sensor membrane electrodes (figure 3.2). By symmetrically supplying voltages to the electrodes of the sensor, the electrostatic transduction nature of the system is exploited to obtain ESS, without actually mechanically 


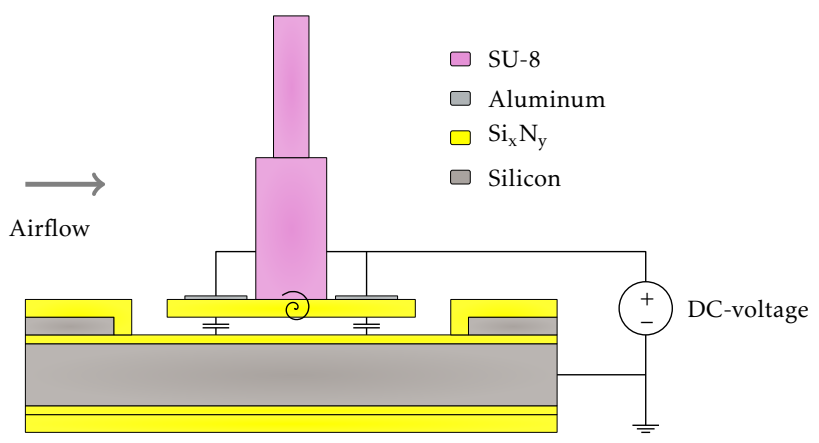

Figure 3.2: Controlling the torsional stiffness by applying dc-bias voltages to the sensor capacitances.

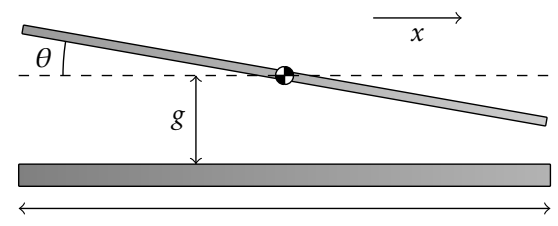

$2 L$

Figure 3.3: Geometry of the angle-dependent rectangular capacitor.

driving the sensor. To model the system's behaviour under application of symmetric dc-bias voltages, we consider the electrostatically induced torque and stiffness which can be calculated from the first and second derivative of the energy in the capacitor with respect to $\theta$ respectively.

Due to the small angles $\theta$ encountered in practice and since the gap is much smaller than both the width $w$ and length of the plates $2 L$, the capacitor is treated as a parallel plate geometry (figure 3.3). The sensor operates in air, for which the relative electric permittivity is assumed to be equal to 1 . Additionally, the two silicon-nitride layers with thicknesses $t_{1}$ and $t_{2}$, and relative permittivity $\epsilon_{\mathrm{r}}$ increase the gap-distance, leading to an effective gap $g_{\text {eff: }}$ :

$$
g_{\text {eff }}=g+\frac{t_{1}}{\epsilon_{r}}+\frac{t_{2}}{\epsilon_{r}} .
$$

The angle dependent capacitance $C(\theta)$ for the rotational sensor using the parallel plate approximation is given by

$$
C(\theta)=\int_{-L}^{L} \frac{\epsilon_{0} w \cos (\theta)}{g_{\text {eff }}-x \tan (\theta)} \mathrm{d} x,
$$

where $x$ is the direction parallel to the plates and $\theta$ is the angle of rotation of the 
upper plate. The solution of this integral is given by

$$
C(\theta)=\epsilon_{0} w \frac{\cos (\theta)}{\sin (\theta)} \ln \left(\frac{g_{\text {eff }}+L \sin (\theta)}{g_{\text {eff }}-L \sin (\theta)}\right) .
$$

Transduction principles are used to find the electrostatic spring softening by an angle-dependent and voltage-controlled capacitor. For this, we use Legendre's transform for the co-energy $E^{\prime}$ of the system, since the capacitor is so-called voltage-controlled:

$$
E^{\prime}(\theta, u)=\frac{1}{2} S_{0} \theta^{2}-\frac{1}{2} u^{2} C(\theta),
$$

where $S_{0}$ is the intrinsic material-based stiffness. The effective stiffness is found by differentiating twice with respect to the rotational angle $\theta$ and keeping the voltage $u$ constant:

$$
S(u, \theta)=\left.\frac{\partial^{2} E^{\prime}}{\partial \theta^{2}}\right|_{u}=S_{0}-\frac{1}{2} u^{2} \frac{\partial^{2} C(\theta)}{\partial \theta^{2}} .
$$

Hence on applying a dc-bias voltage $U_{\mathrm{dc}}$ the total torsional stiffness $S$ becomes

$$
S(u, \theta)=S_{0}-\eta U_{\mathrm{dc}}^{2}, \quad \eta=\frac{1}{2} \frac{\partial^{2} C(\theta)}{\partial \theta^{2}} \approx \frac{2 \epsilon_{0} w L^{3}}{3 g_{\text {eff }}^{3}} .
$$

These expressions state that the total torsional stiffness contains the intrinsic material-based stiffness $S_{0}$ and, under the small rotational angles normally encountered, a time-independent softening term dependent on the applied dc-bias voltage. For frequencies below the sensor's resonance frequency the system shows a larger responsivity. In addition, since the torsional stiffness $S$ is reduced, also the resonance frequency $\omega_{r}$ of the system is affected:

$$
\omega_{r}=\sqrt{\frac{S_{0}-\eta U_{\mathrm{dc}}^{2}}{J}} .
$$

Therefore, the sensor's sensitivity increases with applied dc-bias voltage, whereas the sensor's resonance frequency is reduced simultaneously. For frequencies (far) below the sensor's resonance, we define the achieved gain as

$$
\text { Gain } \approx \frac{S_{0}}{S_{0}-\eta U_{\mathrm{dc}}^{2}}=\frac{1}{1-\kappa U_{\mathrm{dc}}^{2}}, \quad \kappa=\frac{\eta}{S_{0}} .
$$

The geometrical and other properties of the capacitive structure, as used to calculate the theoretical gain and reduction in bandwidth, are listed in table 3.1. 
Table 3.1: Capacitive values for the artificial hair flow sensor.

\begin{tabular}{lll}
\hline Quantity & Symbol & Value \\
\hline Capacitive structures & & \\
\hline Width & $w$ & $95 \mu \mathrm{m}$ \\
Length & $L$ & $90 \mu \mathrm{m}$ \\
Gap (air) & $g$ & $850 \mathrm{~nm}$ \\
Nitride thickness & $t_{1}$ & $1 \mu \mathrm{m}$ \\
Nitride thickness & $t_{2}$ & $200 \mathrm{~nm}$ \\
Relative permittivity & $\epsilon_{\mathrm{r}}$ & 7.5 \\
\hline
\end{tabular}

\subsubsection{Virtual dc-biasing}

In addition to dc-bias voltages, the previously defined expression for the net torsional stiffness $S$ is also valid for time-dependent voltages $u(t)$. In this case we take an ac-bias voltage with amplitude $U_{\mathrm{ac}}$ and frequency $\omega_{\mathrm{ac}}$ :

$$
S \approx S_{0}-\eta\left[U_{\mathrm{ac}} \cos \left(\omega_{\mathrm{ac}} t\right)\right]^{2}=S_{0}-\frac{1}{2} \eta U_{\mathrm{ac}}^{2}-\frac{1}{2} \eta U_{\mathrm{ac}}^{2} \cos \left(2 \omega_{\mathrm{ac}} t\right) .
$$

For frequencies $\omega_{\mathrm{ac}}$ much higher than the system's resonance frequency $\omega_{\text {res }}$, the system will be dominated by inertial effects and show no dependence on the torsional stiffness $S$. As a result, the net torsional stiffness can be approximated by

$$
S \approx S_{0}-\frac{1}{2} \eta U_{\mathrm{ac}}^{2}, \quad \text { if } \quad \omega_{\mathrm{ac}} \ll \omega_{\text {res }} .
$$

Comparing this result with the expression for application of a dc-bias voltage, similar relationships are observed. Therefore, we call this "virtual dc-biasing" using an ac-bias voltage with a high frequency to obtain time-independent ESS.

Furthermore, we define the achieved gain as the ratio between the rotational angle amplitude with and without the application of an ac-bias voltage. It can be approximated for flow frequencies (far) below the sensor's resonance as

$$
\text { Gain } \approx \frac{S_{0}}{S_{0}-\frac{1}{2} \eta U_{\mathrm{ac}}^{2}}=\frac{1}{1-\lambda U_{\mathrm{ac}}^{2}},
$$

where $\lambda$ is an electromechanical parameter defined as

$$
\lambda=\frac{\eta}{2 S_{0}}=\frac{1}{2} \kappa
$$




\subsubsection{Impact on mechanical response}

With the electrostatically affected torsional stiffness $S$ described, its impact on the mechanical response of the artificial hair flow sensor can be described. Taking into account that the drag-torque depends on the relative velocity (i.e. the difference in flow- and hair-velocities), the total system's governing differential equation is according to Humphrey and Barth (2008), with inclusion of the ESS-effect:

$$
\begin{array}{r}
\left(J+J_{\rho}+J_{\mu}\right) \frac{\mathrm{d}^{2} \theta(t)}{\mathrm{d} t^{2}}+\left(R+R_{\mu}\right) \frac{\mathrm{d} \theta(t)}{\mathrm{d} t}+\left(S_{0}-\eta U_{\mathrm{dc}}^{2}\right) \theta(t)= \\
4 \pi \mu G \int_{0}^{L} v_{\mathrm{y}}(t) y \mathrm{~d} y+\left(\frac{\pi \rho d^{2}}{4}-\frac{\pi^{2} \mu G}{g \omega}\right) \int_{0}^{L} \frac{\mathrm{d} v_{\mathrm{y}}(t)}{\mathrm{d} t} y \mathrm{~d} y .
\end{array}
$$

The quantities $J_{\rho}$ and $J_{\mu}$ are often referred to as virtual added mass and $R_{\mu}$ as the virtual added damping (Humphrey and Barth, 2008):

$$
J_{\rho}=\frac{\pi \rho d^{2} L^{3}}{12}, \quad J_{\mu}=-\frac{\pi^{2} \mu G L^{3}}{3 g \omega}, \quad R_{\mu}=\frac{4}{3} \pi \mu G L^{3} .
$$

Using the approach given by Shimozawa et al. (1998) (see also chapter 10 and appendix A), the angular deflection amplitude $\Theta_{m}$ for an oscillating airflow with frequency $\omega$ is given by

$$
\Theta_{\mathrm{m}}=\frac{\sqrt{A^{2}+B^{2}}}{\sqrt{\left[S_{0}-\eta U_{\mathrm{dc}}^{2}-\left(J+J_{\rho}+J_{\mu}\right) \omega^{2}\right]^{2}+\left[\left(R+R_{\mu}\right) \omega\right]^{2}}},
$$

and the phase shift $\phi_{\mathrm{m}}$ with respect to the oscillating airflow as

$$
\phi_{\mathrm{m}}=\arctan \left(\frac{B\left[S_{0}-\eta U_{\mathrm{dc}}^{2}-\left(J+J_{\rho}+J_{\mu}\right) \omega^{2}\right]-A\left(R+R_{\mu}\right) \omega}{A\left[S_{0}-\eta U_{\mathrm{dc}}^{2}-\left(J+J_{\rho}+J_{\mu}\right) \omega^{2}\right]+B\left(R+R_{\mu}\right) \omega}\right),
$$

where

$$
A=\int_{0}^{L}\left|Z_{S}\right| V_{y} y \cos \left(\zeta_{y}+\eta_{\mathrm{S}}\right) \mathrm{d} y, \quad B=\int_{0}^{L}\left|Z_{\mathrm{S}}\right| V_{y} y \sin \left(\zeta_{y}+\eta_{\mathrm{S}}\right) \mathrm{d} y .
$$




\subsubsection{Noise and detection threshold}

An important characteristic of a sensory system is its signal-to-noise ratio (SNR). Generally, when the SNR is equal or higher than one, a system may allow to detect the input signal. Hence, besides the responsivity of the sensor, the noise level of the system determines the detection limit of the system. To find the detection limit of our system, we consider the power spectral density due to the Johnson-Nyquist (thermal) white noise in the system (Gabrielson, 1993):

$$
\overline{T_{\mathrm{n}}^{2}}=4 k_{\mathrm{B}} T_{0}\left(R+R_{\mu}\right),
$$

where $k_{\mathrm{B}}$ is the Boltzmann's constant, $T_{0}$ is the ambient temperature and $\bar{T}_{n}^{2}$ the square of the noise induced equivalent torque per unit of bandwidth. The mechanical transfer function $H(\omega)$ of the system determines the resulting mechanical noise power:

$$
H(\omega)=\frac{1}{-\left(J+J_{\rho}+J_{\mu}\right) \omega^{2}+j\left(R+R_{\mu}\right) \omega+S_{0}-\eta U_{\mathrm{dc}}^{2}} .
$$

The equivalent noise angle $\Theta_{n}$ is found by integrating the noise power over the full spectrum and taking the square root:

$$
\Theta_{\mathrm{n}}=\sqrt{\int_{0}^{\infty} \overline{T_{\mathrm{n}}^{2}}|H(\omega)|^{2} \mathrm{~d} \omega} .
$$

The detection limit $V_{\text {th }}$ is found by dividing the sensor's equivalent noise angle $\Theta_{\mathrm{n}}$ by the rotational angle $\Theta_{\mathrm{m}}$ for a flow of $1 \mathrm{~m} \mathrm{~s}^{-1}$ :

$$
V_{\mathrm{th}}=\frac{\Theta_{\mathrm{n}}}{\left.\Theta_{\mathrm{m}}\right|_{V_{0}=1 \mathrm{~ms}^{-1}}} .
$$

Thermal noise calculations for our hair mechanical system indicate a detection limit for flow sensing of about $85 \mu \mathrm{m} \mathrm{s}^{-1}$ for frequencies within the sensor's bandwidth $\omega_{r}$. This value approaches the detection limit of the cricket cercal hair system, which shows a detection limit down to $30 \mu^{-1} \mathrm{~s}^{-1}$ (Shimozawa et al., 2003).

From a system point of view, the thermal mechanical noise $\bar{T}_{\mathrm{n}}^{2}$ determines the ultimate sensor detection limit, but in practice also other noise source contribute to the system's total noise level. Main contributors are the environmental noise (vibrations in the measurement setup, air perturbations) and especially electrical noise in the measurement setup electronics.

To obtain a model for fitting the membrane displacement $z$ based upon the 
SNR and finding the sensor's detection limit, the power present in the signal and the noise is considered. The signal is assumed to have a linear relationship with respect to the flow velocity amplitude $V_{0}$, given by the coefficient $S_{\mathrm{c}} \cdot N_{\mathrm{c}}$ is a constant representing the total noise, giving for the measured output $z$ :

$$
z=\sqrt{\left(S_{\mathrm{c}} V_{0}\right)^{2}+N_{\mathrm{c}}^{2}}
$$

From this expression the measured detection threshold $V_{\min }$ can be found by equating the noise and signal powers (or alternatively by calculating the intersection value of the asymptotes) which requires only the values for $S_{\mathrm{c}}$ and $N_{\mathrm{c}}$ :

$$
V_{\min }=\frac{N_{\mathrm{c}}}{S_{\mathrm{c}}}
$$

\subsection{Electrostatic spring hardening}

The sensor design of the artificial hair flow sensor allows for reducing the net torsional stiffness $S$. However, by adjusting the flow sensor design by adding interdigitated comb finger structures as described in (Droogendijk et al., 2012), these capacitive structures can be exploited for increasing the system's stiffness electrostatically by electrostatic spring hardening (ESH). Therefore, the hair flow sensor is extended by such structures, where an artist impression of the flow sensor is shown in figure 3.4. Notice that the hair flow sensors will become fully tunable, since the mechanism of ESS remains applicable. A SEM-picture of the fabricated fully tunable hair flow sensors is shown in figure 3.5, where the sensors are designed in a $3 \times 4$ array configuration.

To find the corresponding value of $\eta$ for exploiting ESH, finite element simulations in 2-D by COMSOL ${ }^{\circledR}$ are used. Based on the flow sensor's design, all comb fingers are positioned in the horizontal plane (figure 3.6). The capacitance of a set of capacitive fingers is simulated for different height differences $z$.

The simulation results indicate a capacitance per unit length $C_{\text {sim }}$ with nonzero second derivative with respect to vertical displacement $z$, which can be used to modify the system's torsional stiffness (Adams et al., 1998). Then, to find its impact on the system's torsional stiffness, the capacitance with respect to the hair's rotational angle $C_{\mathrm{ESH}}(\theta)$ is approximated by:

$$
C_{\mathrm{ESH}}(\theta) \approx N_{\mathrm{f}} \int_{L}^{L+\Delta L} C_{\mathrm{sim}}(z \tan (\theta))+C_{\text {sim }}(-z \tan (\theta)) \mathrm{d} z,
$$

where $L$ is the distance to the capacitive structures with respect to the axis of rotation, $\Delta L$ is the overlap in distance between the interdigitated comb fingers, 


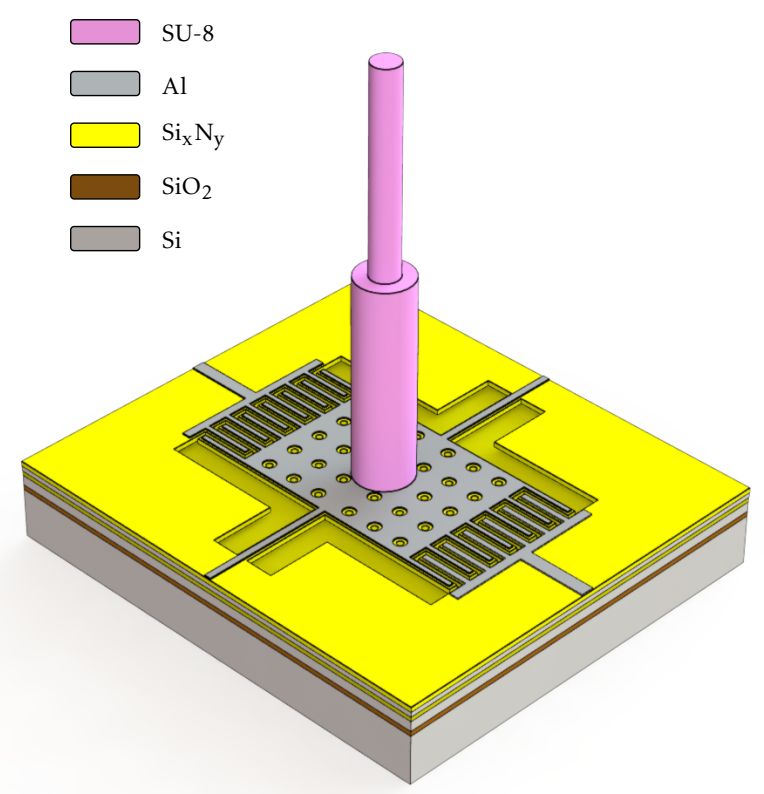

Figure 3.4: Schematic view of the fully tunable hair flow sensor.

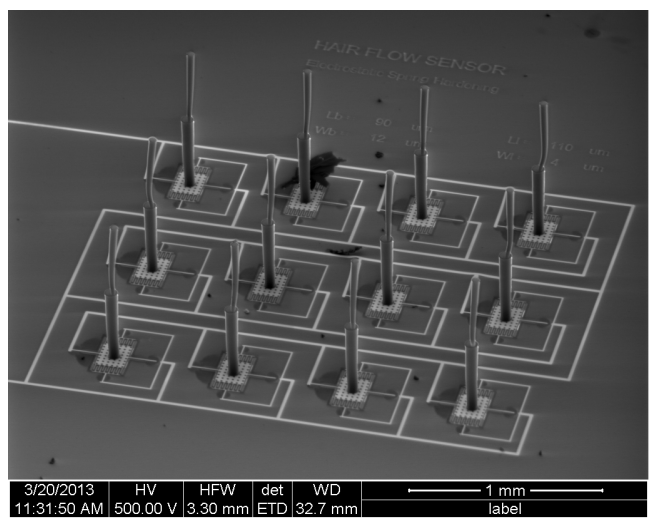

Figure 3.5: Fully tunable hair flow sensors fabricated by surface micromachining and using SU-8 lithography.

and $N_{\mathrm{f}}$ is the number of comb finger pairs at each side. By using the parameters listed in table 3.2 for the fully tunable flow sensor, the capacitive parameter $\eta$ for $\mathrm{ESH}$ is found by taking the second derivative of the parabolic fit shown in figure 3.7, with a corresponding value of $-3.1 \times 10^{-12} \mathrm{Nm} \mathrm{rad}^{-1} \mathrm{~V}^{-2}$, whereas the $\eta$-value for ESS is calculated using (3.7) and found to be $1.7 \times 10^{-10} \mathrm{~N} \mathrm{~m} \mathrm{rad}^{-1} \mathrm{~V}^{-2}$. 


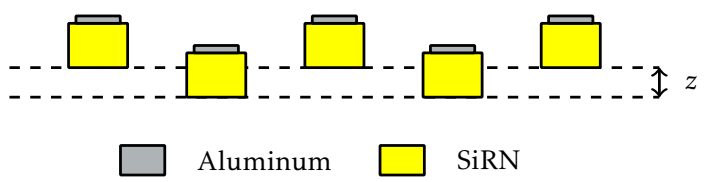

Figure 3.6: Cross-sectional view of the capacitive interdigitated fingers in the (modified) hair flow sensor. The lower fingers are attached to the movable membrane, the upper fingers are anchored to the substrate.

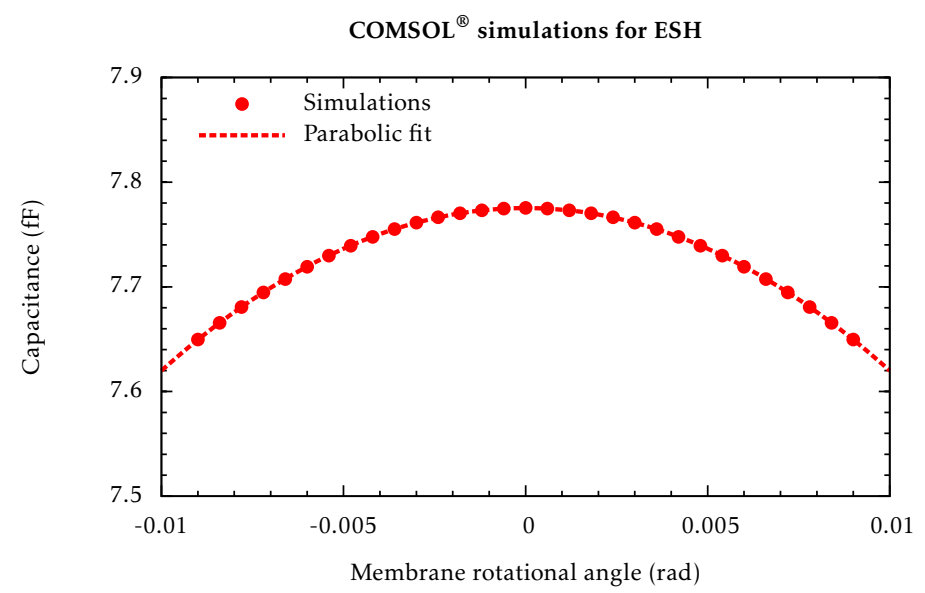

Figure 3.7: Calculated capacitance for ESH, based on finite element 2-D simulations of the comb drive structures.

Table 3.2: Capacitive values for the fully tunable hair flow sensor.

\begin{tabular}{lll}
\hline Quantity & Symbol & Value \\
\hline Capacitive structures & & \\
\hline Width & $w$ & $150 \mu \mathrm{m}$ \\
Length & $L$ & $100 \mu \mathrm{m}$ \\
Plate length & $\Delta L$ & $50 \mu \mathrm{m}$ \\
Number of fingers & $N_{\mathrm{f}}$ & 12 \\
Gap (air) & $g$ & $1.54 \mu \mathrm{m}$ \\
Nitride thickness & $t_{1}$ & $1.2 \mu \mathrm{m}$ \\
Nitride thickness & $t_{2}$ & $150 \mathrm{~nm}$ \\
Relative permittivity & $\epsilon_{\mathrm{r}}$ & 7.5 \\
\hline
\end{tabular}




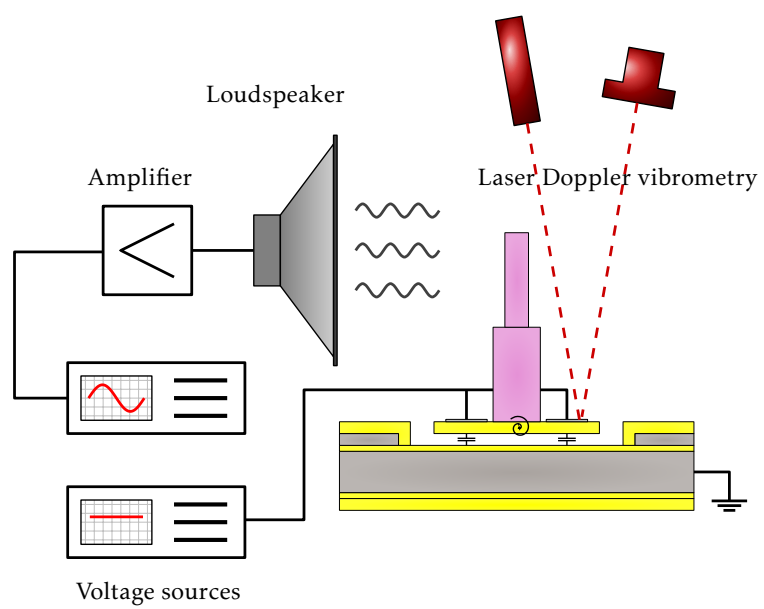

Figure 3.8: Optical measurement setup to determine the membrane displacement of the artificial hair flow sensor.

\subsection{Experimental}

\subsubsection{Electrostatic spring softening}

\section{Optical measurements}

ESS-experiments using optics to measure the sensor's response are performed using the setup shown in figure 3.8. A waveform generator (Agilent 33220A-001) is used to produce a sinusoidal signal at a frequency $f_{\mathrm{a}}$ that is supplied to an amplifier. This amplifier drives a loudspeaker (Visaton WS $17 \mathrm{E}$ ) to generate the oscillating airflow. A $10 \mathrm{~cm}$ Si wafer is glued on the loudspeaker cone to achieve a flow profile that can be well described by (very) near field theory (de Bree et al., 2004). Another voltage source (Delta Elektronika - Power Supply E 030-1) is used to supply the dc-bias voltage to the top electrodes. The bottom electrode is grounded as is the measurement setup. The sensor rotational angle $\theta$ is derived from laser Doppler vibrometry using a Polytec MSA-400 (Polytec, 2005).

First, the mechanical transfer of the hair sensory system was determined (Figure 3.9). During this measurement, a dc-bias voltage $U_{\mathrm{dc}}$ of $2.5 \mathrm{~V}$ was used, giving an increase in sensitivity of about $80 \%$ for frequencies within the sensor's bandwidth. Also lowering of the resonance frequency $\omega_{r}$ is observed (about 20\%). Further, the relationship between dc-bias voltage $U_{\mathrm{dc}}$ and its effect on the mechanical transfer of the system was measured (Figure 3.10). For frequencies of both $100 \mathrm{~Hz}$ and $300 \mathrm{~Hz}$ a non-linear relationship is observed, which is also expected 


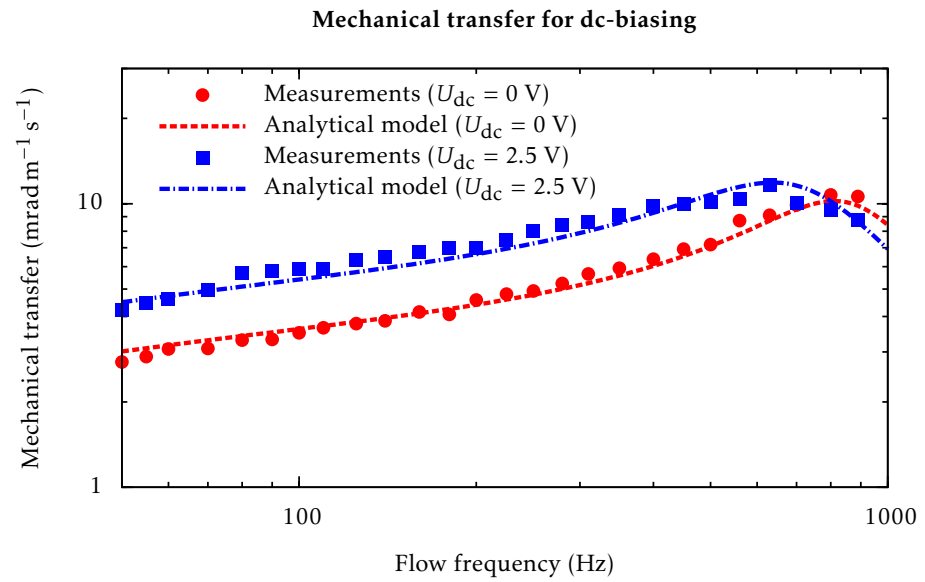

Figure 3.9: Enhancing the mechanical transfer of the hair-flow sensor by applying a dc-bias voltage.

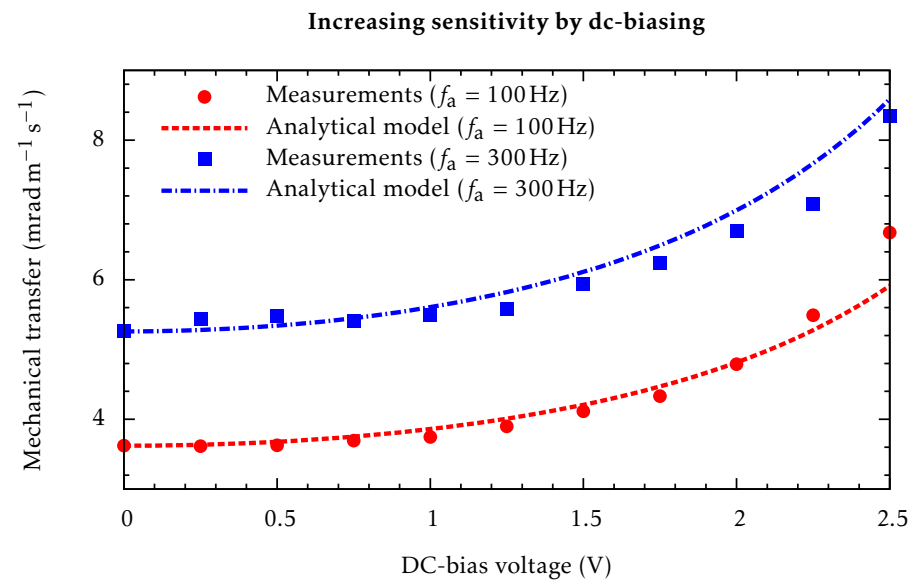

Figure 3.10: Sensitivity increases non-linearly with respect to the applied dc-bias voltage.

by the quadratic nature of electrostatic actuation (3.7). Optical measurements for the mechanical transfer are in good agreement with modelling and it is shown clearly that dc-biasing leads to a larger sensitivity below the sensor's resonance frequency (figure 3.9). The resonance frequency of the system is also lowered, as predicted by (3.8).

The experimental results on application of an ac-bias voltage by exploiting virtual dc-biasing to increase the amplitude of the rotational angle are given 


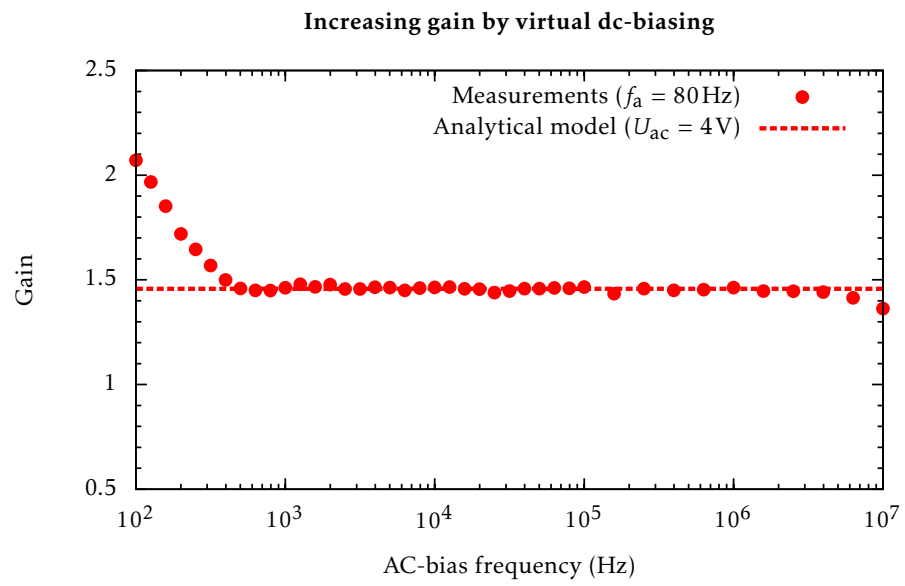

Figure 3.11: Increased gain for applying an ac-bias voltage.

Threshold lowering by dc-biasing

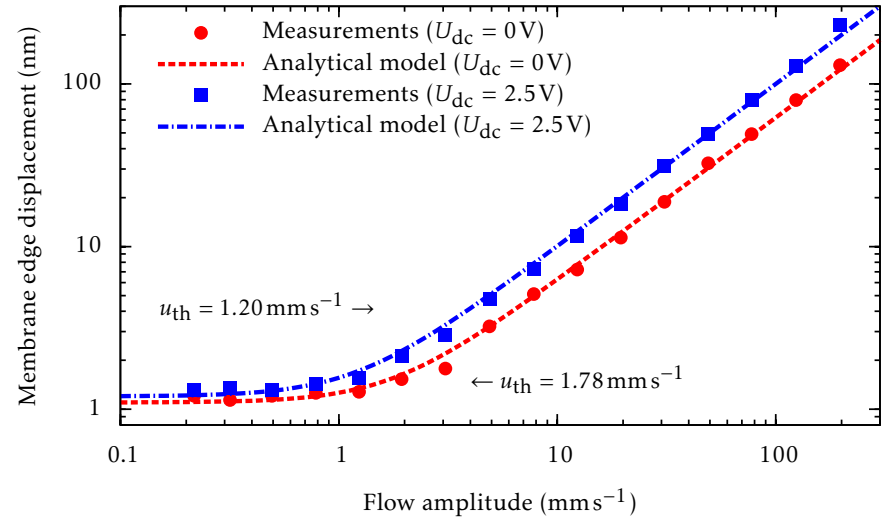

Figure 3.12: Threshold lowering by dc-biasing at $110 \mathrm{~Hz}$, giving an improvement of more than $30 \%$ for $U_{\mathrm{d} c}=2.5 \mathrm{~V}$.

in figure 3.11. During the experiment, a flow with a constant amplitude and a frequency of $80 \mathrm{~Hz}$ was used. An ac-bias voltage was supplied to the capacitive structures, for which the amplitude $U_{\text {ac }}$ was set to $4 \mathrm{~V}$ and the frequency $\omega_{\text {ac }}$ was varied.

The detection limit of the flow sensory system was measured with and without application of a dc-bias voltage for a harmonic flow of $110 \mathrm{~Hz}$. Specifying the detection limit as the flow-velocity amplitude at which the signal-to-noise ratio 


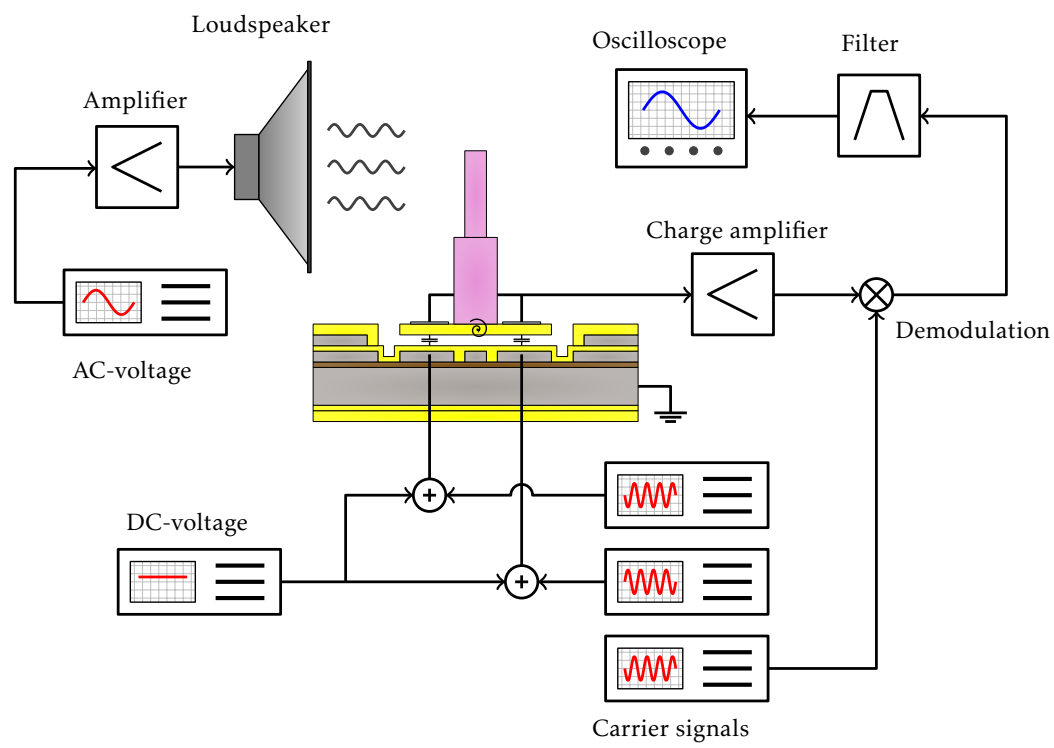

Figure 3.13: Capacitive measurement setup for determining the membrane displacement of the hair-flow sensor.

(SNR) is equal to unity, our sensory system shows an improvement of more than $30 \%$ (figure 3.12).

\section{Capacitive measurements}

To investigate the effective improvement of ESS on sensor operation capacitive measurements were carried out as well. For these experiments, a different hair flow sensor was used. Although the hair mechanics are identical to the sensor used during optical measurements, the used sensor is a so-called single hair flow sensor, fabricated using silicon-on-insulator technology (Dagamseh et al., 2010).

A schematic of the experimental setup as used for experiments on capacitive operation is shown in figure 3.13. The part of the setup generating the oscillating airflow is identical to the part shown in figure 3.8. However, the dc-bias voltages are supplied here to the sensor's bottom electrodes, together with the carrier signals (at $1 \mathrm{MHz}$ ) needed for capacitive read-out. The top electrode is connected to a charge amplifier, and after demodulation and filtering (Stanford SR 650) the sensor's response can be monitored on an oscilloscope (Agilent DS1024).

Using the capacitive read-out, the detection limit of the flow sensory system was measured with and without application of a dc-bias voltage for harmonic flows 


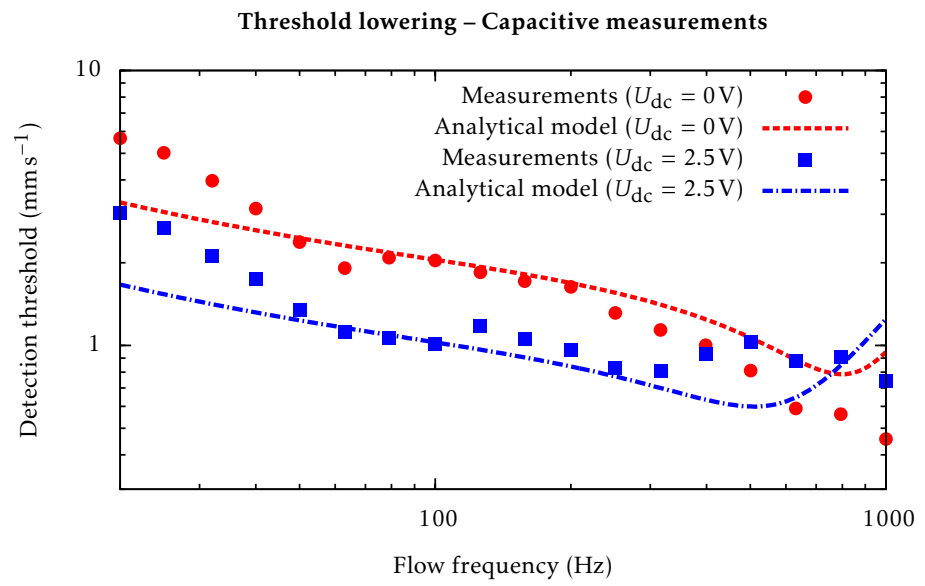

Figure 3.14: Capacitive measurements for threshold lowering on application of a dc-bias voltage.

(figure 3.14). Rather than for one frequency, as in the optical measurements (figure $3.12)$, the detection limit was determined for frequencies within a range of 20 $1000 \mathrm{~Hz}$, using (3.23). Based on the thermal noise calculations from section 3.2.3, the noise floor of the sensory system is considered to be dominated by electronic noise.

First, the output rms-voltage of the system was measured without flow, giving the value for $N_{\mathrm{c}}$. Then, the airflow amplitude was increased until the rms-voltage was increased to $\sqrt{2} N_{\mathrm{c}}$. As a result, the required flow amplitude gives the detection limit of the used hair flow sensor at the flow frequency used. The experimental data indicates that for frequencies within a range of $50-200 \mathrm{~Hz}$ the detection limit is improved by about $6 \mathrm{~dB}$ on applying a dc-bias voltage of $2.5 \mathrm{~V}$.

\subsubsection{Electrostatic spring hardening}

To investigate the impact of ESH on the fully tunable hair flow sensor, the frequency response of the sensor was measured optically using laser Doppler vibrometry (Polytec, 2005). In contrast to the measurements shown in figure 3.9, the hair flow sensor was not subjected to an harmonic airflow, but was driven electrostatically to demonstrate the sensor's tuning capability. By choosing a chirpbased voltage for actuation in a push-pull configuration, both magnitude and phase response were measured, with the results shown in figure 3.15.

Both ESS and ESH conditions were applied to the fully tunable hair flow sensor, 

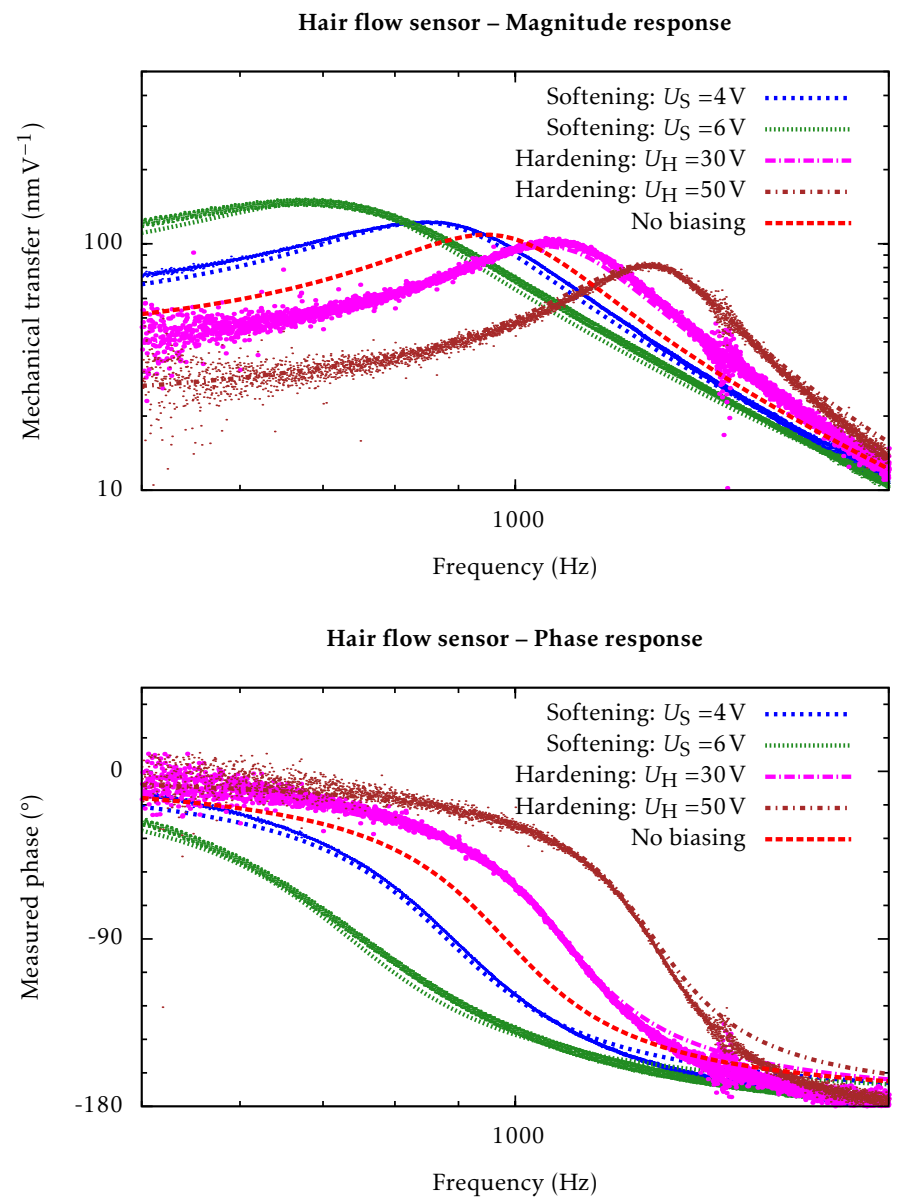

Figure 3.15: Optically measured mechanical transfer of the hair flow sensor using electrostatic actuation.

to verify its adaptability to lower and higher frequencies. Together with the measurements also the theoretical response of the electromechanical system is shown. The values for $\eta$ for fitting the data were found to be $-3.0 \times 10^{-12} \mathrm{Nm} \mathrm{rad}^{-1} \mathrm{~V}^{-2}$ for ESH and $1.15 \times 10^{-10} \mathrm{~N} \mathrm{~m} \mathrm{rad}^{-1} \mathrm{~V}^{-2}$ for ESS, given the values for the system parameters $S_{0}=9.5 \times 10^{-9} \mathrm{Nm} \mathrm{rad}^{-1}, R=5.7 \times 10^{-13} \mathrm{Nm} \mathrm{srad}^{-1}$ and $J=2.5 \times 10^{-16} \mathrm{~kg} \mathrm{~m}^{2}$. Due to the used push-pull mode for electrostatic actuation, also the theoretical response for no biasing is shown by the red dashed line in figure 3.15, since electrostatic actuation inherently results in biasing of the sensor. We observe that the bandwidth of the hair flow sensors becomes indeed fully tunable by applying 


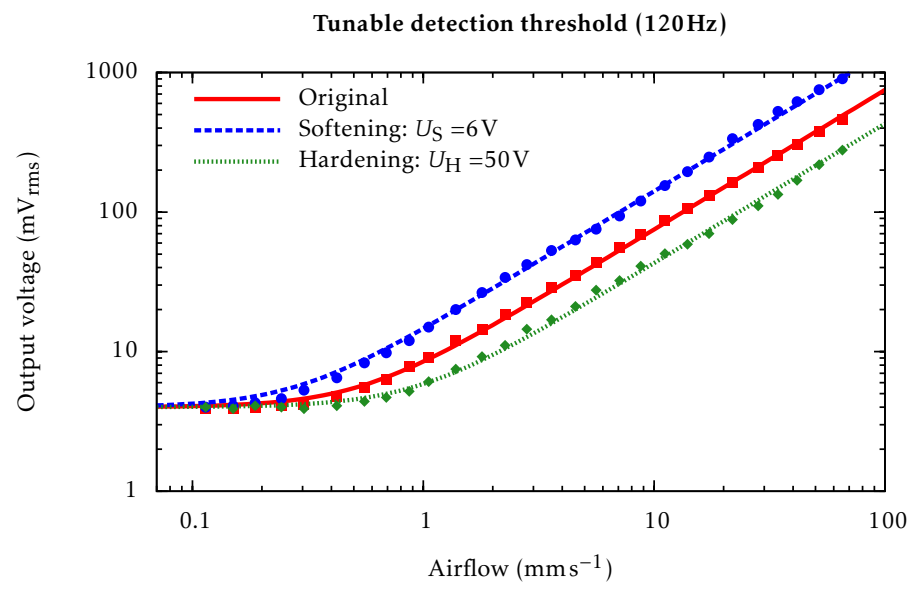

Figure 3.16: Capacitively measured output voltage versus airflow amplitude at a frequency of $120 \mathrm{~Hz}$.

a dc-bias voltage for either ESS or ESH conditions, and the analytical model and measurements are in good agreement.

\section{Linearity and detection threshold}

Using the measurement setup shown in figure 3.13, the response of the fully tunable hair flow sensor was measured without biasing, by applying ESS (6V) and by applying ESH $(50 \mathrm{~V})$. An airflow was applied with a frequency of $120 \mathrm{~Hz}$ and the amplitude was varied within a range of approximately three decades. The obtained measurement results are shown in figure 3.16, together with the analytical curves based on (3.23) to determine the sensor's detection threshold.

We observe that for all measurements a good agreement is observed between the analytical model and the experimental data. Following figure 3.16, the measured noise level is dominated by electronics noise, since only the detection threshold is affected by applying ESS or ESH, while the noise level remains the same. From the experimental data shown in figures 3.15 and 3.16, the characteristics for the fully tunable hair flow sensor are calculated and shown in table 3.3. Generally, by using this $3 \times 4$ array hair flow sensor measurements of airflows with amplitudes in the range of sub $\mathrm{mm} \mathrm{s}^{-1}$ are demonstrated, with a full scale error in the order of $1 \%$. Also, the measured dynamic range was measured, where it is expected that the real value will be higher due to limitations of the used loudspeaker (Visaton WS $17 \mathrm{E}$ ) for generating airflows with larger amplitudes. 
Table 3.3: Experimental values of the fully tunable biomimetic hair flow sensor.

\begin{tabular}{lllll}
\hline Quantity & Symbol & ESS (6V) & No biasing & ESH (50 V) \\
\hline Mechanical parameters & & & & \\
\hline Quality factor & $Q$ & 2.0 & 2.7 & 3.6 \\
Resonance frequency & $f_{0}$ & $737 \mathrm{~Hz}$ & $981 \mathrm{~Hz}$ & $1.31 \mathrm{kHz}^{-1}$ \\
Threshold $(120 \mathrm{~Hz})$ & $V_{\min }$ & $0.28 \mathrm{~mm} \mathrm{~s}^{-1}$ & $0.53 \mathrm{~mm} \mathrm{~s}^{-1}$ & $0.93 \mathrm{~mm} \mathrm{~s}^{-1}$ \\
Full scale error $(120 \mathrm{~Hz})$ & $\epsilon_{\mathrm{FS}}$ & $1.3 \%$ & $0.6 \%$ & $1.2 \%$ \\
Dynamic range $(120 \mathrm{~Hz})$ & $\mathrm{DR}$ & $\geq 47.4 \mathrm{~dB}$ & $\geq 41.8 \mathrm{~dB}$ & $\geq 36.9 \mathrm{~dB}$ \\
\hline
\end{tabular}

\subsection{Discussion}

\subsubsection{Electrostatic spring softening}

Fitting the model from (3.16) and evaluating the constant $\eta$ defined in (3.7), a value of $5.0 \times 10^{-10} \mathrm{Nm} \mathrm{rad}^{-1} \mathrm{~V}^{-2}$ is found, which is comparable to the calculated value of $5.1 \times 10^{-10} \mathrm{Nm} \mathrm{rad}^{-1} \mathrm{~V}^{-2}$ for a gap $g$ of $800 \mathrm{~nm}$. Although higher dc-bias voltages result in further increased sensitivity, there exists a trade-off between increased sensitivity and stability, because of the pull-in effect - the torsional stiffness $S$ becomes very small or negative. The calculated pull-in voltage in case of dc-biasing is about $4.4 \mathrm{~V}$ and for ac-biasing the amplitude is about $6.2 \mathrm{~V}$.

Using an ac-bias voltage indeed also results in gain of the sensor response (Figure 3.11). Generally, for an ac-bias amplitude of $4 \mathrm{~V}$ and for bias frequencies higher than $600 \mathrm{~Hz}$ - which is around the system's resonance - the gain is found to be approximately 1.45 over a frequency range of four decades. For lower frequencies, the spring softening term containing twice the ac-bias frequency $\omega_{\text {ac }}$ cannot be neglected anymore and parametric amplification effects have to be taken into account (Rugar and Grütter, 1991; Droogendijk et al., 2011). Frequencies above $1 \mathrm{MHz}$ show a slight decrease with increasing frequency, which is caused by output limitations of the used waveform generator.

The required $\eta$ value for fitting the model from (3.12) in figure 3.11 is about $3.6 \times 10^{-10} \mathrm{~N} \mathrm{~m} \mathrm{rad}^{-1} \mathrm{~V}^{-2}$. Although this value is in the same order of magnitude as the one found when using a dc-bias voltage, the value is about $30 \%$ smaller than the value expected from theory and dc-bias experiments. A possible explanation for this deviation is the presence of charges built-in the silicon-nitride layers (Wibbeler et al., 1998), resulting in a dc-voltage offset and therefore altering the expected theoretical gain. Calculations indicate that a built-in voltage of about $1 \mathrm{~V}$ can explain this difference in the observed gain values. 
Depending on the origin of the noise present in the sensory system, ESS can result in a lower sensory threshold and thus help to improve the detection limit of the sensor. Our current flow sensors have a detection limit of about $1 \mathrm{~mm} \mathrm{~s}^{-1}$ for oscillating airflows at about $100 \mathrm{~Hz}$, but based on thermal noise calculations it is expected that this can be lowered significantly on improvement of the interfacing electronics. Our optical measurements show that ESS decreases the detection limit of the sensor by increasing the responsivity, whereas the noise floor level only shows a slight increase (figure 3.12). The main noise contributor is the limited resolution of the laser Doppler vibrometer, which is specified to be in the order of $1 \mu \mathrm{m} \mathrm{s}^{-1}$. This resolution corresponds to a displacement of about $1.5 \mathrm{~nm}$, which is comparable to our measured noise level.

Measurements for threshold lowering (figure 3.14) indicate that the application of a dc-bias voltage of $2.5 \mathrm{~V}$ gives an improvement in detection limit of up to $6 \mathrm{~dB}$ for a wide range of frequencies. The fitted model shows good agreement with the experimental data for frequencies within a range of $50-200 \mathrm{~Hz}$. The experimental data shows that for frequencies below $50 \mathrm{~Hz}$ our model predictions and the experimental data show poorer agreement than at higher frequencies. We suspect that at these lower frequencies the flow-to-torque part of our model, which is based upon Stokes expressions for viscous drag forces (Stokes, 1851), is not entirely suitable anymore. This effect is less pronounced in figure 3.9 where, due to limitations in the Laser Doppler Vibrometer, the lower frequency response could not reliably be determined.

Although both the experimental data and the theoretical model show a decrease in the sensor's bandwidth (i.e. resonance frequency), there is a clear difference between model and measurements for frequencies above $300 \mathrm{~Hz}$. A possible explanation is the coupling between the induced electromagnetic field of the used loudspeaker, which is placed very close to the hair flow sensor (to benefit from very near field conditions (de Bree et al., 2004)), and the electronics for capacitive read-out. Furthermore, due to imperfections in the used electronics for capacitive read-out (e.g. non-linear mixing of frequencies), we were not able to perform capacitive experiments on virtual dc-biasing.

\subsubsection{Electrostatic spring hardening}

Although the impact of ESH generally leads to a lower responsivity, it still offers advantages with respect to bandwidth and stability. In contrast to the mechanism of ESS, wherein a trade-off exists between responsivity and stability, a system under ESH conditions will be stable for every chosen dc-bias voltage, since the system's torsional stiffness $S$ theoretically cannot become zero or negative. Though, 
in case the dc-bias voltage on the ESH structures is very high (hundreds of V), snap-in of the comb drive fingers may occur. Further, the bandwidth of a system subjected to ESH will be increased, depending on the applied voltage. Another consequence of implementing ESH is the increased quality factor, as observed in figure 3.15. This increase can be an advantage when the frequencies of interest are present in a narrow frequency-band for which mechanical amplification can be achieved.

\subsubsection{Overall}

The combination of ESS and ESH can be used to adapt the sensor's performance to the actual needs by either adjusting for a large bandwidth and higher quality factor, or a high responsivity and lower detection threshold. However, although theoretically high gain of the flow signal is possible, a trade-off exists with respect to instability by pull-in. Therefore, the achieved gain by dc-biasing is practically limited to about a factor of 2-3.

More general, implementing ESS and ESH for the enhancement of the sensor's performance is not only applicable to our biomimetic hair flow sensory system, but is applicable to other energy-buffering two-port transducers as well. Since the used theory is based entirely on the nature of energy buffering transducers, equivalents of stiffness control for e.g. increasing sensitivity can also be realized for piezoelectric, electromagnetic and other types of energy buffering transducers. The application of ESS and ESH could be useful in case of (e.g. robotic) applications when specific information about the environment is unknown. By using such a mechanism, a system offers the freedom to be adapted to the environment, resulting in enhanced performance.

\subsection{Conclusions}

Applying a dc-bias voltage on the capacitors of our biomimetic hair flow sensors gives both an increase in responsivity for frequencies within the sensor's bandwidth and lowers the sensory threshold for ESS. Also a high frequency acbias voltage was shown to improve the responsivity of the system. Generally, we can detect lower flow velocities by adapting our flow sensors by applying bias voltages to the sensor's capacitive structures. By applying a dc-bias voltage on ESH capacitive structures, the bandwidth is increased at the cost of reduced responsivity of the hair flow sensor. 


\section{References}

Adams, S. G., Bertsch, F. M., Shaw, K. A., and MacDonald, N. C. (1998). Independent tuning of linear and nonlinear stiffness coefficients. J. Microelectromech. S., $7(2): 172-180$.

Dagamseh, A. M. K., Bruinink, C. M., Droogendijk, H., Wiegerink, R. J., Lammerink, T. S. J., and Krijnen, G. J. M. (2010). Engineering of biomimetic hair-flow sensor arrays dedicated to high-resolution flow field measurements. In Proc. IEEE Sensors 2010, pp. 2251-2254, Waikoloa, HI, United States.

de Bree, H.-E., Svetovoy, V. B., Raangs, R., and Visser, R. (2004). The very near field. Theory, simulations and measurements of sound pressure and particle velocity in the Very Near Field. In Proc. $11^{\text {th }}$ Int. Congress on Sound and Vibration, St. Petersburg, Russia.

Droogendijk, H., Bruinink, C. M., Sanders, R. G. P., and Krijnen, G. J. M. (2011). Non-resonant parametric amplification in biomimetic hair flow sensors: Selective gain and tunable filtering. Appl. Phys. Lett., 99(21):213503.

Droogendijk, H., Groenesteijn, J., Haneveld, J., Sanders, R. G. P., Wiegerink, R. J., Lammerink, T. S. J., Lötters, J. C., and Krijnen, G. J. M. (2012). Parametric excitation of a micro Coriolis mass flow sensor. Appl. Phys. Lett., 101(22):223511.

Floris, J., Izadi, N., Jaganatharaja, R. K., Wiegerink, R. J., Lammerink, T. S. J., and Krijnen, G. J. M. (2007). Adaptation for frequency focusing and increased sensitivity in biomimetic flow sensors using electrostatic spring softening. In Proc. Transducers'07, pp. 1267-1270, Lyon, France.

Gabrielson, T. B. (1993). Mechanical thermal noise in micromachined acoustic and vibration sensors. IEEE Trans. Electron. Devices, 40:903-909.

Howard, J. and Hudspeth, A. J. (1987). Mechanical relaxation of the hair bundle mediates adaptation in mechanoelectrical transduction by the bullfrog's saccular hair cell. PNAS, 84:3064-3068.

Humphrey, J. A. C. and Barth, F. (2008). Medium flow-sensing hairs: biomechanics and models. Adv. Insect Phys., 34:1-80.

Krijnen, G. J. M., Dijkstra, M., van Baar, J. J., Shankar, S. S., Kuipers, W. J., de Boer, R. J. H., Altpeter, D., Lammerink, T. S. J., and Wiegerink, R. J. (2006). MEMS based hair flow-sensors as model systems for acoustic perception studies. Nanotechnology, 17:S84-S89. 
Polytec (2005). MSA-400 Micro System Analyzer. Polytec, Waldbronn, Germany.

Rugar, D. and Grütter, P. (1991). Mechanical parametric amplification and thermomechanical noise squeezing. Phys. Rev. Lett., 67:699-702.

Shimozawa, T., Kumagai, T., and Baba, Y. (1998). Structural scaling and functional design of the cercal wind-receptor hairs of cricket. J. Comp. Physiol. A, 183:171186.

Shimozawa, T., Murakami, J., and Kumagai, T. (2003). Cricket wind receptors: thermal noise for the highest sensitivity known. In Sensors and sensing in biology and engineering, pp. 145-159. Springer.

Stokes, G. G. (1851). On the effect of the internal friction of fluids on the motion of pendulums. Trans. Cambr. Phil. Soc., 9:1-141.

Wibbeler, J., Pfeifer, G., and Hietschold, M. (1998). Parasitic charging of dielectric surfaces in capacitive microelectromechanical systems (MEMS). Sens. Act. A: Phys., 71:74-80. 


\section{4 \\ Non-resonant parametric amplification}

\subsection{Introduction}

To further improve the performance of the artificial hair flow sensors and implement adaptive filtering, we make use of non-resonant parametric amplification (NRPA). By modulating the torsional stiffness of the sensory system we can make it selective for arbitrary flow frequencies and simultaneously achieve significant amplification of the sensor response.

Parametric amplification exploits complex interactions between excitatory signals in which amplitude, frequency and phase play important roles in the entanglement of the signals determining the overall response. Rugar and Grütter (1991) presented the concept of parametric amplification in electromechanical systems, in which they discuss a system operating at resonance and, with proper choices of parameters, exhibiting amplification of the input of the system. The mechanism of parametric amplification has been exploited by several research groups with applications in torsional resonators (Carr et al., 2000; Baskaran and

This chapter is based on "Non-resonant parametric amplification in biomimetic hair flow sensors: Selective gain and tunable filtering” by H. Droogendijk, C. M. Bruinink, R. G. P. Sanders, and G. J. M. Krijnen, published in Appl. Phys. Lett., 99(21): 213503, (2011), and "Advantages of electrostatic spring hardening in biomimetic hair flow sensors” by H. Droogendijk, M. J. de Boer, R. G. P. Sanders, and G. J. M. Krijnen, submitted to New J. Phys., (2014). 


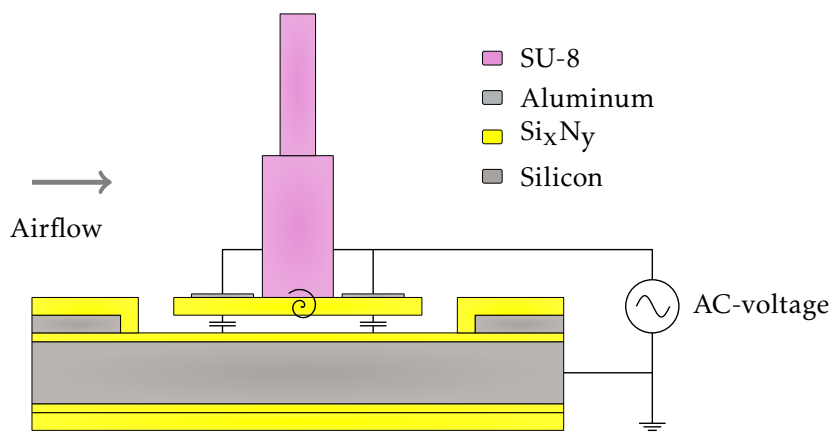

Figure 4.1: Controlling the torsional stiffness by applying ac-bias voltages to the sensor capacitances.

Turner, 2003), resonant micro-mirrors (Kim et al., 2013), thermal resonant sensors (Ono et al., 2005), MEMS gyroscopes (Sharma et al., 2011), mass flow sensors (Groenesteijn et al., 2013) and Lorentz force based magnetometers (Thompson and Horsley, 2011). In contrast, we do not operate at the sensor's resonance, but still achieve electromechanical filtering and selective gain.

\subsection{Theory and modelling}

To exploit parametric amplification in the hair-based flow sensor, an ac-bias voltage is symmetrically supplied to the electrodes, inducing balanced electrostatic forces (figure 4.1). As a result, the torsional stiffness $S$ is modulated giving $S(t)$.

The resulting time-dependent stiffness $S(t)$ is calculated from the geometry of the rotating parallel plate capacitor. Applying transduction principles, the electrostatically softened torsional stiffness $S(t)$ on application of an ac-bias voltage with amplitude $U_{\mathrm{p}}$, angular frequency $\omega_{\mathrm{p}}$ and phase $\phi_{\mathrm{p}}$ is given by

$$
S(t)=S_{0}-\frac{1}{4} U_{\mathrm{p}}^{2} \frac{\partial^{2} C}{\partial \theta^{2}}-\frac{1}{4} U_{\mathrm{p}}^{2} \cos \left(2 \omega_{\mathrm{p}} t+2 \phi_{\mathrm{p}}\right) \frac{\partial^{2} C}{\partial \theta^{2}} .
$$

As a result, the torsional stiffness $S(t)$ is dependent on frequency, phase and amplitude of the applied ac-bias voltage, further referred to as pump signal. Under the small rotational angles normally encountered, the total torsional stiffness $S(t)$ contains the intrinsic material-based stiffness $S_{0}$, a time-independent softening term and frequency and phase-dependent softening term. 


\subsubsection{Simulations}

For specific conditions, the solution of (3.1) under ac-biasing conditions can be obtained using analytical methods and approximations. However, as a more general approach to modelling our electromechanical system, allowing for quick investigation of e.g. non-resonant interactions, a strong pump and a low quality factor (our flow sensors are nearly critically damped systems), numerical simulations are used to determine the system behaviour. Using the ode4 explicit fixed-step solver from Simulink show that significant amplification is achieved when periodic softening occurs at twice the flow frequency $f_{\mathrm{a}}$ of the system, requiring frequency matching of the pump to the harmonic airflow $\left(f_{\mathrm{a}}=f_{\mathrm{p}}\right)$.

To study the system performance and to determine its selectivity, the analysis is divided in two areas: matching the frequencies of flow and pump $\left(f_{\mathrm{p}}=f_{\mathrm{a}}\right)$ and non-matching of these frequencies $\left(f_{\mathrm{p}} \neq f_{\mathrm{a}}\right)$. Furthermore, the pump phase $\phi_{\mathrm{p}}$ is varied between 0 and 360 degrees while keeping the pump amplitude $U_{\mathrm{p}}$ and flow frequency $f_{\mathrm{a}}$ fixed.

Non-resonant parametric amplification (NRPA) can give selective gain or attenuation, depending on the pump frequency $f_{\mathrm{p}}$ and pump phase $\phi_{\mathrm{p}}$. Equal frequencies for flow and pump $\left(f_{\mathrm{p}}=f_{\mathrm{a}}\right)$ give coherency in torque and spring softening, for which the pump phase determines whether the system will show relative amplification or attenuation. Therefore, it is possible to realize a very sharp band pass/stop filter, depending on the pump settings.

\subsubsection{Harmonic balancing method}

To find an analytical approximation for the amplitude of the frequency components at which the flow information is present, the harmonic balancing method (HBM) is used (Harish et al., 2009). By balancing terms containing $\pm \omega_{\mathrm{a}}$ and $\pm\left(2 \omega_{\mathrm{p}} \pm \omega_{\mathrm{a}}\right)$, and truncating the series for higher order terms, the rotational angle amplitudes for the retained frequencies are found.

Consider the second order differential equation from (3.1) describing the hair sensor's mechanics. For convenience, the airflow induced torque $T(t)$ and timedependent torsional stiffness $S(t)$ can be expressed as

$$
T(t)=\frac{T_{0}}{2}\left[e^{j \omega_{\mathrm{a}} t}+e^{-j \omega_{\mathrm{a}} t}\right], \quad S(t)=S_{0}-\frac{1}{2} \frac{\partial^{2} C}{\partial \theta^{2}} u(t)^{2} .
$$

The ac-bias voltage is a harmonic voltage with amplitude $U_{\mathrm{p}}$, phase $\phi_{\mathrm{p}}$ and frequency $\omega_{\mathrm{p}}$ : 


$$
u(t)=\frac{U_{\mathrm{p}}}{2}\left[e^{j\left(\omega_{\mathrm{p}} t+\phi_{\mathrm{p}}\right)}+e^{-j\left(\omega_{\mathrm{p}} t+\phi_{\mathrm{p}}\right)}\right] .
$$

Squaring the expression for $u(t)$ :

$$
u(t)^{2}=\frac{U_{\mathrm{p}}^{2}}{2}+\frac{U_{\mathrm{p}}^{2}}{4} e^{j 2 \phi_{\mathrm{p}}} e^{j 2 \omega_{\mathrm{p}} t}+\frac{U_{\mathrm{p}}^{2}}{4} e^{-j 2 \phi_{\mathrm{p}}} e^{-j 2 \omega_{\mathrm{p}} t} .
$$

In this work, the ac-bias voltage $u(t)$ will be applied to the ESS capacitive structures, whereas a dc-bias voltage $U_{\mathrm{h}}$ will be applied to the ESH capacitive structures. As a result, the torsional stiffness $S(t)$ can be expressed as

$$
S(t)=S_{0}-\frac{1}{2} \eta_{\mathrm{S}} U_{\mathrm{p}}^{2}+\eta_{\mathrm{H}} U_{\mathrm{h}}^{2}-\frac{1}{4} \eta_{\mathrm{S}} U_{\mathrm{p}}^{2} e^{j 2 \phi_{\mathrm{p}}} e^{j 2 \omega_{\mathrm{p}} t}-\frac{1}{4} \eta_{\mathrm{S}} U_{\mathrm{p}}^{2} e^{-j 2 \phi_{\mathrm{p}}} e^{-j 2 \omega_{\mathrm{p}} t},
$$

in which $\eta_{\mathrm{S}}$ represents the geometrical constant defined in (3.7) and $\eta_{\mathrm{H}}$ is derived by differentiating (3.25) twice. This result shows that the net torsional stiffness $S(t)$ contains five terms: three that are frequency-independent, one at frequency $2 \omega_{\mathrm{p}}$ and one at frequency $-2 \omega_{\mathrm{p}}$.

\section{Matched frequencies}

In the case of matched frequencies, the pump frequency equals the flow frequency $\left(\omega_{\mathrm{p}}=\omega_{\mathrm{a}}\right)$. Then, due to the product of $S(t)$, given by $(4.5)$, and $u(t)^{2}$, given by (4.4), the rotational angle amplitude $\theta(t)$ will also contain frequency components at $3 \omega_{a}$ :

$$
\theta(t)=\theta_{1} e^{j \omega_{\mathrm{a}} t}+\theta_{2} e^{-j \omega_{\mathrm{a}} t}+\theta_{3} e^{j\left(3 \omega_{\mathrm{a}}\right) t}+\theta_{4} e^{-j\left(3 \omega_{\mathrm{a}}\right) t},
$$

where higher frequencies are neglected. This system of equations can be approximated as a linear system of coupled differential equations, one for each of the frequencies in (4.6). The differential equations are given by

$$
-\omega_{i}^{2} J \theta_{i}+j \omega_{i} R \theta_{i}+[S(t) \theta(t)]_{\omega_{i}}=\frac{T_{0}}{2}\left[\delta\left(\omega_{i}-\omega_{\mathrm{a}}\right) e^{j \omega_{\mathrm{a}} t}+\delta\left(\omega_{i}+\omega_{\mathrm{a}}\right) e^{-j \omega_{\mathrm{a}} t}\right],
$$

where $[S(t) \theta(t)]_{\omega_{i}}$ implies the terms at frequency $\omega_{i}$. The Kronecker delta function $(\delta)$ restricts the driving to the frequency of the airflow $\left(\omega_{\mathrm{a}}\right)$. For convenience, we introduce the shorthand notations:

$$
a_{1}=\frac{1}{4} \eta_{\mathrm{S}} U_{\mathrm{p}}^{2} e^{j 2 \phi_{\mathrm{p}},} \quad a_{2}=\frac{1}{4} \eta_{\mathrm{S}} U_{\mathrm{p}}^{2} e^{-j 2 \phi_{\mathrm{p}},} \quad c=\frac{T_{0}}{2} .
$$


Furthermore, we define the frequencies:

$$
\begin{array}{ll}
\omega_{1}=\omega_{\mathrm{a}}, & \omega_{3}=3 \omega_{\mathrm{a}}, \\
\omega_{2}=-\omega_{1}, & \omega_{4}=-\omega_{3} .
\end{array}
$$

Additionally, we define for the system-related terms involving the system parameters $J, R$ and $S_{0}$ :

$$
h(\omega)=-\omega^{2} J+j \omega R+S_{0}-\frac{1}{2} \eta_{\mathrm{S}} U_{\mathrm{p}}^{2}+\eta_{\mathrm{H}} U_{\mathrm{h}}^{2}, \quad h_{i}=h\left(\omega_{i}\right),
$$

wherein contributions by added air damping and mass are neglected (Humphrey and Barth, 2008). On collecting terms at equal frequencies and neglecting frequencies higher than $3 \omega_{\mathrm{a}}$, we find

$$
\begin{array}{ll}
@ \omega_{1}: & h_{1} \theta_{1}-a_{1} \theta_{2}-a_{2} \theta_{3}=c, \\
@ \omega_{2}: & h_{2} \theta_{2}-a_{2} \theta_{1}-a_{1} \theta_{4}=c, \\
@ \omega_{3}: & h_{3} \theta_{3}-a_{1} \theta_{1}=0, \\
@ \omega_{4}: & h_{4} \theta_{4}-a_{2} \theta_{2}=0 .
\end{array}
$$

By solving this set of linear equations, we find for the amplitude $\theta_{1}$ at flow frequency $\omega_{1}$ :

$$
@ \omega_{1}: \theta_{1}=c \times \frac{1+\frac{a_{1}}{h_{2}}\left[1-\frac{a_{2}}{h_{4}}\right]}{h_{1}-a_{1} a_{2}\left[\frac{h_{1}}{h_{3} h_{4}}+\frac{1}{h_{2}}+\frac{1}{h_{3}}-\frac{a_{1} a_{2}}{h_{2} h_{3} h_{4}}\right]} .
$$

\section{Non-matched frequencies}

A similar approach can be made for the case when $\omega_{\mathrm{a}} \neq \omega_{\mathrm{p}}$, where the rotational angle $\theta(t)$ is assumed to have the form

$$
\begin{aligned}
\theta(t)= & \theta_{1} e^{j \omega_{\mathrm{a}} t}+\theta_{2} e^{-j \omega_{\mathrm{a}} t}+\theta_{3} e^{j\left(2 \omega_{\mathrm{p}}-\omega_{\mathrm{a}}\right) t}+ \\
& \theta_{4} e^{-j\left(2 \omega_{\mathrm{p}}-\omega_{\mathrm{a}}\right) t}+\theta_{5} e^{j\left(2 \omega_{\mathrm{p}}+\omega_{\mathrm{a}}\right) t}+\theta_{6} e^{-j\left(2 \omega_{\mathrm{p}}+\omega_{\mathrm{a}}\right) t} .
\end{aligned}
$$

For convenience, the following shorthand notations are used:

$$
a_{0}=\frac{1}{4} \eta_{\mathrm{S}} U_{\mathrm{p}}^{2}, \quad c=\frac{T_{0}}{2} .
$$

Furthermore, we define the frequencies: 


$$
\begin{array}{lll}
\omega_{1}=\omega_{\mathrm{a}}, & \omega_{3}=2 \omega_{\mathrm{p}}-\omega_{\mathrm{a}}, & \omega_{5}=2 \omega_{\mathrm{p}}+\omega_{\mathrm{a}}, \\
\omega_{2}=-\omega_{1}, & \omega_{4}=-\omega_{3}, & \omega_{6}=-\omega_{5} .
\end{array}
$$

Following the analysis given in chapter 5 , the amplitude $\theta_{1}$ at the flow frequency $\omega_{1}$ is given by

$$
\text { @ } \omega_{1}: \theta_{1}=c \times \frac{1}{g_{1}-a_{0}^{2}\left[\frac{1}{g_{4}}+\frac{1}{g_{5}}\right]},
$$

where $g_{i}$ contains the for the system-related contributions:

$$
g(\omega)=-\omega^{2} J+j \omega R+S_{0}-\frac{1}{2} \eta_{\mathrm{S}} U_{\mathrm{p}}^{2}+\eta_{\mathrm{H}} U_{\mathrm{h}}^{2}, \quad g_{i}=g\left(\omega_{i}\right)
$$

Now, consider the case in which the flow frequency $\omega_{\mathrm{a}}$ and pump frequency $\omega_{\mathrm{p}}$ are not-matched, but are close to each other:

$$
\omega_{\mathrm{a}}=\omega_{\mathrm{p}}+\Delta \omega, \quad \text { with } \quad \Delta \omega \ll \omega_{\mathrm{p}}
$$

A response will be induced at a non-flow frequency $\omega_{3}$, which is close to the pump frequency:

$$
\omega_{3}=\omega_{\mathrm{p}}-\Delta \omega,
$$

for which the response is given by

$$
@ \omega_{3}: \theta_{3}=c \times \frac{a_{0}}{g_{3}} \times \frac{1}{g_{2}-a_{0}^{2}\left[\frac{1}{g_{3}}+\frac{1}{g_{6}}\right]} \text {. }
$$

\subsection{Experimental}

Experiments for NRPA were performed using the setup shown in figure 4.2. A waveform generator (Agilent 33220A-001) is used to produce a sinusoidal signal at a frequency $f_{\mathrm{a}}$ that is supplied to an amplifier. This amplifier drives a loudspeaker (Visaton WS $17 \mathrm{E}$ ) to generate the harmonic airflow. A silicon wafer is glued onto the loudspeaker cone to achieve a flow profile that is well described by (very) near field theory (de Bree et al., 2004).

The ac-pump voltage supplied to the top electrodes is generated by a second waveform generator (Agilent 33220A-001) that is synchronized with the first one to control the pump phase $\phi_{\mathrm{p}}$ with respect to the airflow signal. The sensor 


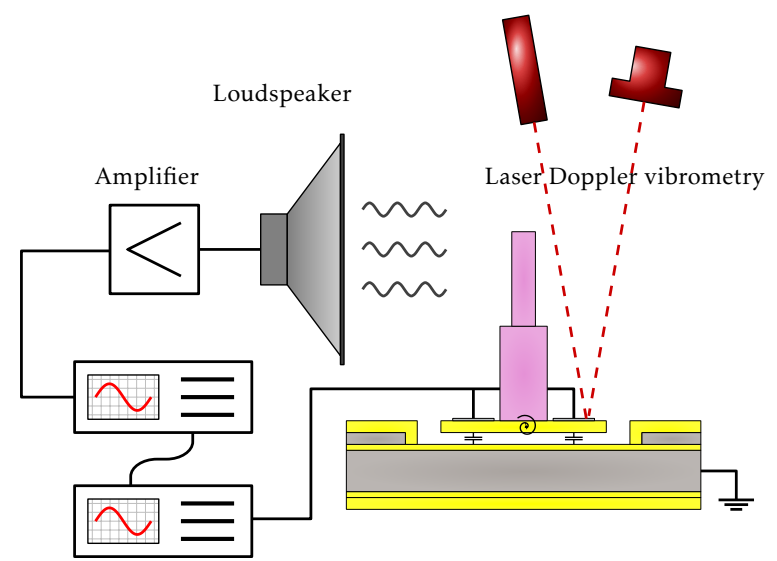

Synchronized waveform generators

Figure 4.2: Measurement setup for determining the rotational angle of the hair flow sensor.

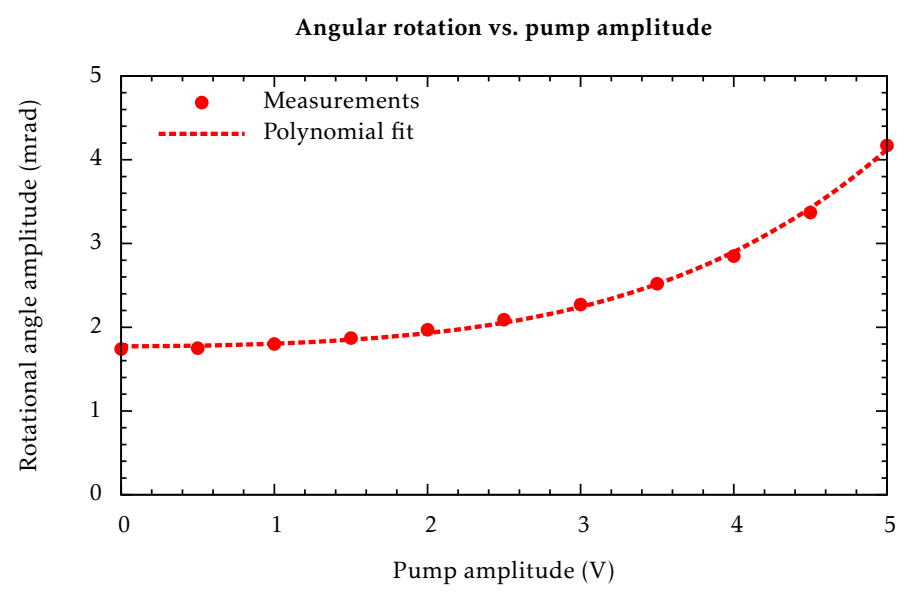

Figure 4.3: Amplitude of the periodic hair rotation angle as function of the pump amplitude $U_{\mathrm{p}}$. The flow frequency $f_{\mathrm{a}}$ was equal to the pump frequency $f_{\mathrm{p}}(200 \mathrm{~Hz})$ and the pump phase $\phi_{\mathrm{p}}$ was set to the value giving maximum gain.

rotational angle $\theta$ is derived from laser Doppler vibrometry using a Polytec MSA400.

Experiments confirm that significant amplification of the sensor's response to the flow signal can be achieved for a suitable choice of pump parameters (figure 4.3). Here, the pump frequency $f_{\mathrm{p}}$ was set equal to the flow frequency $f_{\mathrm{a}}$ $(200 \mathrm{~Hz})$. The pump amplitude $U_{\mathrm{p}}$ was varied and the phase $\phi_{\mathrm{p}}$ was fixed at the 


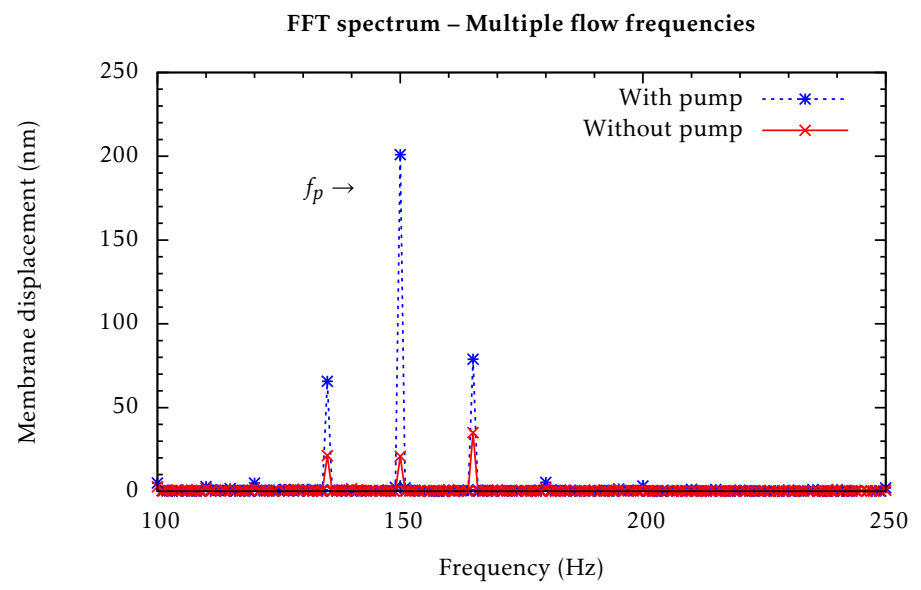

Figure 4.4: Measured gain of about $20 \mathrm{~dB}$ for the airflow frequency component at $150 \mathrm{~Hz}$ determined by FFT. The pump is fixed at $f_{\mathrm{p}}=150 \mathrm{~Hz}$ with an amplitude of $5 \mathrm{~V}$.

value that produced maximum gain at $f_{\mathrm{p}}=f_{\mathrm{a}}$. A non-linear relationship between the rotational angle $\theta$ and the amplitude $U_{\mathrm{p}}$ of the applied pump voltage is observed, which is also expected by the quadratic nature of electrostatic actuation.

To investigate selective gain and filtering by PA, three frequencies were applied simultaneously to the loudspeaker (using arbitrary waveform generation), resulting in a periodic airflow with three frequency components $(135 \mathrm{~Hz}, 150 \mathrm{~Hz}$ and $165 \mathrm{~Hz}$ ). The pump frequency $f_{\mathrm{p}}$ was set to $150 \mathrm{~Hz}$, with a pump amplitude $U_{\mathrm{p}}$ of $5 \mathrm{~V}$. In figure 4.4 , the spectrum of the hair rotational angle is shown for both the situations with and without pumping. Clearly, all three frequency components show gain, the maximum gain of about $20 \mathrm{~dB}$ experienced for the $150 \mathrm{~Hz}$ (matched) frequency. However, the ratio between the components changes when PA is applied to the system, resulting in a selective gain-advantage of $9 \mathrm{~dB}$ or more. This is in agreement with theory, which predicts frequency-independent amplification $(135 \mathrm{~Hz}, 150 \mathrm{~Hz}$ and $165 \mathrm{~Hz})$ by the second term in (4.1) and frequency-dependent amplification $(150 \mathrm{~Hz})$ by the third term in $(4.1)$, both for frequencies below resonance.

To determine mixing of frequencies and the residual membrane displacement $z_{\text {res }}$ when using PA, the FFT amplitude spectra for both matching and non-matching flow and pump frequencies are examined (figure 4.5). For these measurements, the pump amplitude $U_{\mathrm{p}}$ is set to $5 \mathrm{~V}$, the pump phase $\phi_{\mathrm{p}}$ was set to the value producing maximum gain, and the pump frequency $f_{\mathrm{p}}$ is fixed at $150 \mathrm{~Hz}$. Then, the spectrum is measured both in absence and presence of 


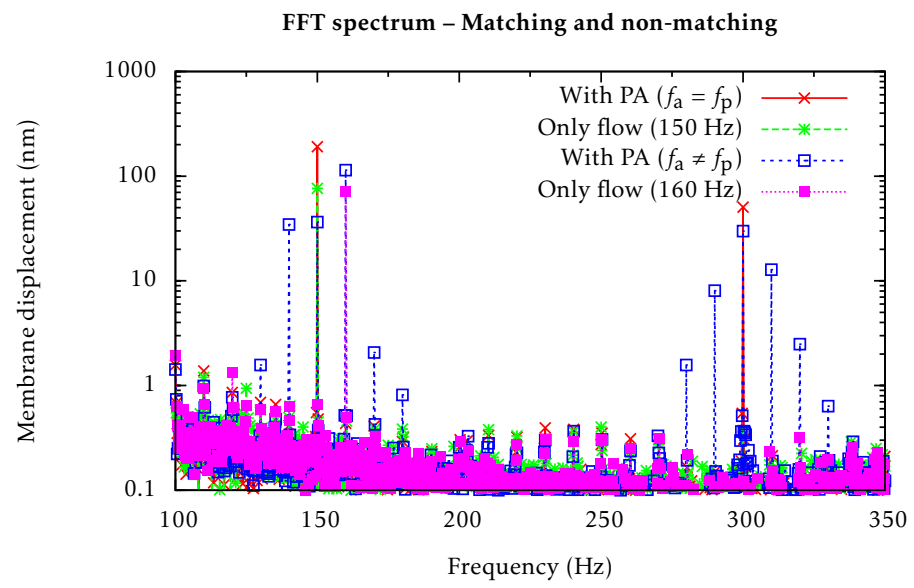

Figure 4.5: Amplitude spectrum for frequency matched (top) and mismatched (bottom) cases, with the pump fixed at $150 \mathrm{~Hz}$, the phase value set to the value giving maximum amplification, and an amplitude of $5 \mathrm{~V}$.

oscillating airflow (for cases $f_{a}=150 \mathrm{~Hz}$ and $f_{a}=160 \mathrm{~Hz}$ ). Similar to figure 4.4, by considering the increase in membrane displacement $z_{\mathrm{amp}}$, selective gain is observed for matching frequencies $(150 \mathrm{~Hz}$, indicated by crosses, figure 4.5 top). For both cases with pump signal (crosses and open squares), the spectrum exhibits frequency components at and around twice the pump frequency $\left(2 f_{\mathrm{p}}=300 \mathrm{~Hz}\right)$, as a result of vertical vibrations of the sensor's membrane (due to limited vertical stiffness and residual charges built-in the silicon-nitride layers) in combination with parametric mixing. Although there is a non-negligible contribution of the parametric pump at $150 \mathrm{~Hz}$ in absence of a frequency matched flow (figure 4.5 bottom, open square at $150 \mathrm{~Hz}$ ), the gain achieved by pumping at the matched frequency (figure $4.5 \mathrm{top}$, cross at $150 \mathrm{~Hz}$ ) is significantly larger, confirming the applicability of parametric amplification for selective gain and filtering.

\subsubsection{Impact of ESH on NRPA}

The experiments for demonstrating selective gain and tunable filtering were performed using the artificial hair flow sensor based on the fabrication process of (Bruinink et al., 2009). To investigate the impact of ESH on NRPA, the fully tunable hair flow sensor is used. By considering (4.5), the ratio of the frequencyindependent terms of $S(t)$ with respect to the frequency-dependent terms can be changed by applying a dc-voltage $U_{\mathrm{H}}$. Using the optical measurement setup shown in figure 4.2 and the addition of a voltage source (Delta Elektronika - Power 


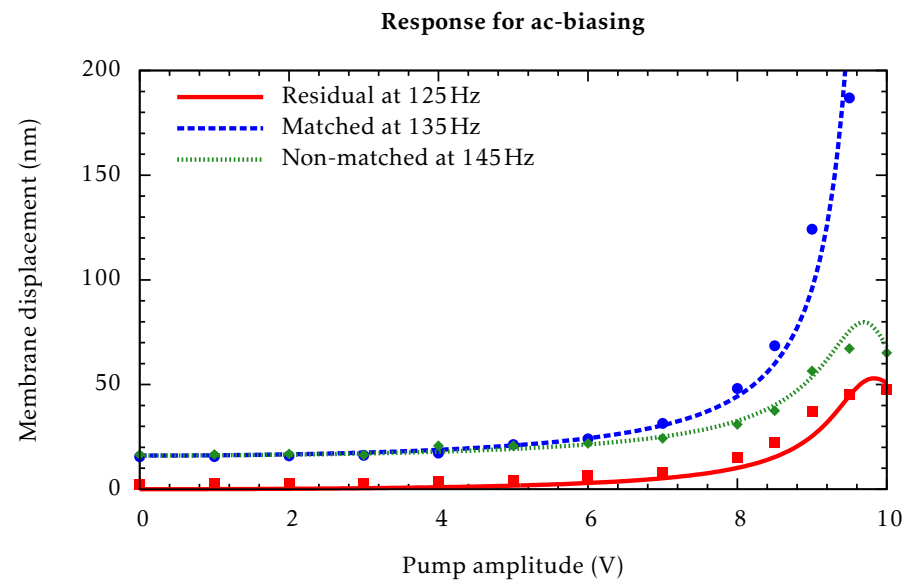

Figure 4.6: Optically measured displacement as function of the pump amplitude $U_{\mathrm{p}}$. Points represent experimental data, lines exhibit the theoretical model using HBM.

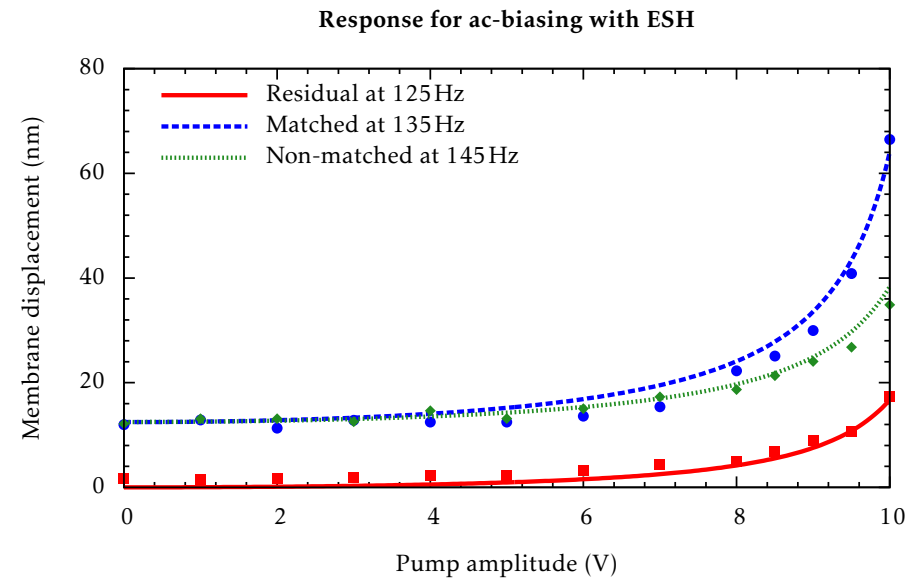

Figure 4.7: Optically measured displacement for ESH as function of the pump amplitude $U_{\mathrm{p}}$. Points represent experimental data, lines exhibit the theoretical model using HBM.

Supply E 0300-0.1), experiments were conducted by using an airflow consisting of two frequencies with equal amplitudes $(135 \mathrm{~Hz}$ and $145 \mathrm{~Hz})$. The pump frequency was set to $135 \mathrm{~Hz}$ and the pump amplitude was varied between $0 \mathrm{~V}$ and $10 \mathrm{~V}$.

First, the response of the hair mechanical system was optically measured without applying $\mathrm{ESH}\left(U_{\mathrm{H}}=0 \mathrm{~V}\right)$. The experimental results are shown in figure 4.6 for three frequencies $(125 \mathrm{~Hz}, 135 \mathrm{~Hz}$ and $145 \mathrm{~Hz})$, together with the theoretical 


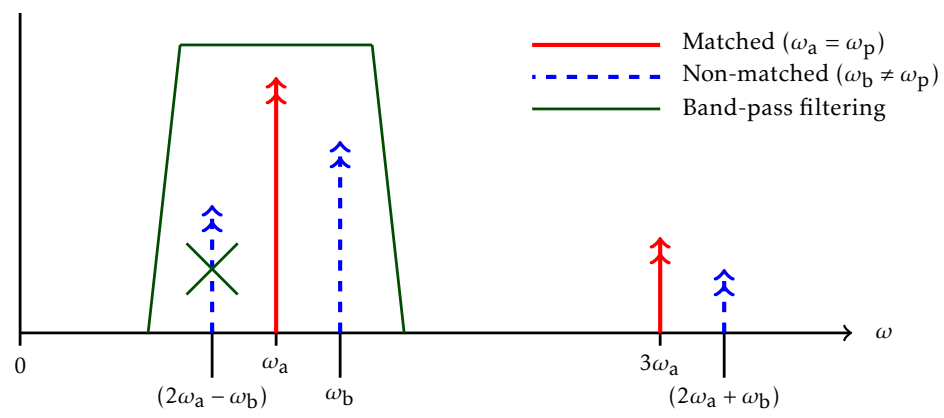

Figure 4.8: Illustration of measured frequencies by exploiting non-resonant parametric amplification. The incoming airflow consists of two frequencies $\left(\omega_{\mathrm{a}}\right.$ and $\left.\omega_{\mathrm{b}}\right)$.

responses for $125 \mathrm{~Hz}$ by (4.20) for the residual component due to parametric mixing, $135 \mathrm{~Hz}$ by (4.12) for the matched case and $145 \mathrm{~Hz}$ by (4.16) for the nonmatched case.

By increasing the dc-bias voltage on the ESH capacitive structures to $30 \mathrm{~V}$, a similar measurement has been conducted. The obtained results are shown in figure 4.7, together with the theoretical responses for the frequencies of $125 \mathrm{~Hz}$, $135 \mathrm{~Hz}$ and $145 \mathrm{~Hz}$. For both figures 4.6 and 4.7 we observe that there is good agreement between the analytical models based on the harmonic balancing method and the experimental data.

\subsection{Discussion}

Measurements for NRPA indicate that applying an ac-bias voltage to the sensor's capacitive structures for read-out offer the possibility for achieving selective gain and tunable filtering (figure 4.4). Although the appliance of multiple flow frequencies leads to matched and non-matched conditions in case of an ac-bias voltage with a fixed frequency, also responses are induced at non-flow frequencies, as shown in figure 4.6. As predicted by the analysis of the NRPA-system these residual component can be expected at the frequencies indicated in figure 4.8. In this figure, the pump frequency is set to $\omega_{\mathrm{a}}$, giving a response for a flow at frequency $\omega_{\mathrm{a}}$ at both $\omega_{\mathrm{a}}$ and $3 \omega_{\mathrm{a}}$, shown in red. A flow with frequency $\omega_{\mathrm{b}}$ will give responses at frequencies $\omega_{\mathrm{b}}, 2 \omega_{\mathrm{a}}-\omega_{\mathrm{b}}$ and $2 \omega_{\mathrm{a}}+\omega_{\mathrm{b}}$. For resolving the frequencies present in the airflow, the frequencies $3 \omega_{\mathrm{a}}$ and $2 \omega_{\mathrm{a}}+\omega_{\mathrm{b}}$ can be filtered using a band-pass filter. As a result, only the undesired residual component at frequency $2 \omega_{\mathrm{a}}-\omega_{\mathrm{b}}$ will remain.

Then by adding a dc-bias voltage of $30 \mathrm{~V}$ to the ESH structures, the response at 


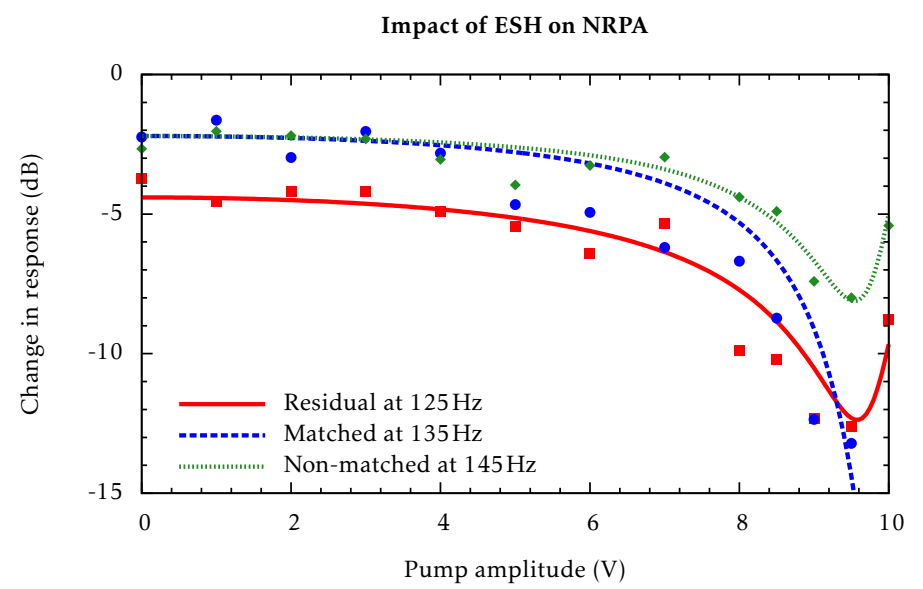

Figure 4.9: The impact of ESH on non-resonant parametric amplification as function of the pump amplitude $U_{\mathrm{p}}$. Points represent experimental data from figures 4.6 and 4.7, lines exhibit the theoretical model using HBM. By exploiting ESH, the residual frequency component is suppressed and the gain for both matched and non-matched frequencies is reduced.

both flow and non-flow frequencies is changed with respect to the case without ESH (figure 4.7). By dividing the responses of all three frequencies for ESH by the response without ESH, the results shown in figure 4.9 are obtained. We observe that when applying ESH to a system subjected to NRPA, the induced response at the so-called residual component can be reduced with $13 \mathrm{~dB}$. Simultaneously, the response of the non-matched frequency component can be reduced by about $8 \mathrm{~dB}$, while the response of the matched flow frequency will be reduced by more than $13 \mathrm{~dB}$. As a result, the residual component can be reduced at the cost of a slightly reduced non-matched response and significantly reduced matched-response. Thus, the filter will exhibit less selective gain, but remains tunable.

Another advantage of application of ESH in combination with NRPA is the ability to achieve selective gain in a larger bandwidth compared to the case without ESH. To illustrate this effect, the selective gain for our flow sensor system - the ratio of matched frequency response and non-matched frequency response - is calculated for the case without ESH with a pump amplitude of 7V (figure 4.6) and for the case with ESH $\left(U_{\mathrm{h}}=30 \mathrm{~V}\right)$ with a pump amplitude of $8 \mathrm{~V}$ (figure 4.7). These values have been chosen such that both systems exhibit comparable selective gain of about $2 \mathrm{~dB}$ at low frequencies. Then, using our HBM-based models, the expected selective gain versus flow frequency is calculated for both cases and shown in figure 4.10. We observe that on application of ESH selective gain can 


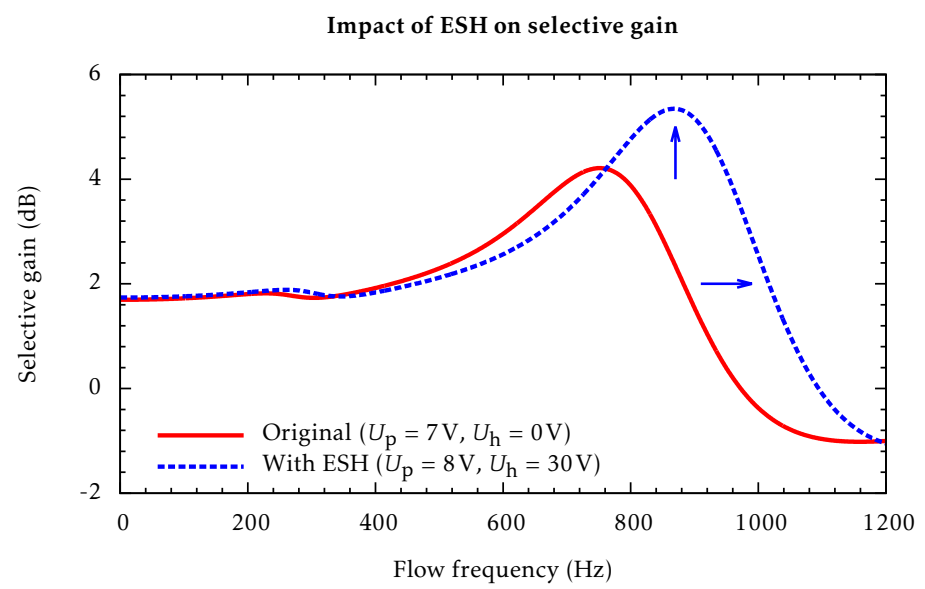

Figure 4.10: The impact of ESH on selective gain versus the flow frequency. The lines exhibit the theoretical model using HBM based on the experimental data from figures 4.6 and 4.7 .

be achieved within a larger bandwidth, while retaining a gain of $2 \mathrm{~dB}$ for low frequencies. Additionally, for high frequencies the selective gain is even increased (blue dashed line) compared to the case without applying ESH (red solid line).

\subsubsection{Overall}

By replacing the dc-bias voltage at the ESS capacitive structures with an ac-bias voltage and matching frequencies of flow and voltage high gain values can be achieved while retaining the sensor's bandwidth. Although these high gain factors are tunable by choosing a judicious voltage, information on frequency and phase of the surrounding airflow is required in order to realize matching of frequencies and finding the optimal phase for achieving optimal amplification of the flow signal. Hence, this method allows for measurement of flow signals from which one has knowledge a priori. This can, for example, be the case when observing modulated flows (e.g., for acoustic communication purposes or dealing with rotary machines).

\subsection{Conclusions}

Non-resonant parametric amplification and filtering has been demonstrated in biomimetic hair flow sensors. By selecting appropriate values for the ac-pump 
voltage, selective gain and filtering is achieved. The responsivity to the incoming airflow can be improved by $20 \mathrm{~dB}$, while having large selectivity with respect to non-matched frequency signals. Also, we argued that the bandwidth of the sensor on exploiting NRPA can be increased for selective gain and tunable filtering.

\section{References}

Baskaran, R. and Turner, K. L. (2003). Mechanical domain coupled mode parametric resonance and amplification in a torsional mode micro electro mechanical oscillator. J. Micromech. Microeng., 13:701-707.

Bruinink, C. M., Jaganatharaja, R. K., de Boer, M. J., Berenschot, J. W., Kolster, M. L., Lammerink, T. S. J., Wiegerink, R. J., and Krijnen, G. J. M. (2009). Advancements in technology and design of biomimetic flow-sensor arrays. In Proc. MEMS 2009, pp. 152-155.

Carr, D. W., Evoy, S., Sekaric, L., Craighead, H. G., and Parpia, J. M. (2000). Parametric amplification in a torsional microresonator. Appl. Phys. Lett., 77(10):1545-1547.

de Bree, H.-E., Svetovoy, V. B., Raangs, R., and Visser, R. (2004). The very near field. Theory, simulations and measurements of sound pressure and particle velocity in the Very Near Field. In Proc. $11^{\text {th }}$ Int. Congress on Sound and Vibration, St. Petersburg, Russia.

Groenesteijn, J., Droogendijk, H., Wiegerink, R. J., Lammerink, T. S. J., Lötters, J. C., Sanders, R. G. P., and Krijnen, G. J. M. (2013). Parametric amplification in a micro Coriolis mass flow sensor: reduction of power dissipation without loss of sensitivity. In Proc. IEEE Sensors 2013, pp. 1626-1629, Baltimore, MD, United States.

Harish, K. M., Gallacher, B. J., Burdess, J. S., and Neasham, J. A. (2009). Experimental investigation of parametric and externally forced motion in resonant MEMS sensors. J. Micromech. Microeng., 19:15-21.

Humphrey, J. A. C. and Barth, F. (2008). Medium flow-sensing hairs: biomechanics and models. Adv. Insect Phys., 34:1-80.

Kim, J., Kawai, Y., Inomata, N., and Ono, T. (2013). Parametrically driven resonant micro-mirror scanner with tunable springs. In Proc. MEMS 2013, pp. 580-583, Taipei, Taiwan. 
Ono, T., Wakamatsu, H., and Esashi, M. (2005). Parametrically amplified thermal resonant sensor with pseudo-cooling effect. J. Micromech. Microeng., 15:22822288.

Rugar, D. and Grütter, P. (1991). Mechanical parametric amplification and thermomechanical noise squeezing. Phys. Rev. Lett., 67:699-702.

Sharma, M., Sarraf, E. H., and Cretu, E. (2011). Parametric amplification/damping in MEMS gyroscopes. In Proc. MEMS 2011, pp. 617-620, Cancun, Mexico.

Thompson, M. J. and Horsley, D. A. (2011). Parametrically amplified z-axis Lorentz force magnetometer. J. Microelectromech. S., 20(3):702-710. 
REFERENCES 


\section{5 \\ Electromechanical amplitude modulation}

\subsection{Introduction}

Exploiting the electrostatic transduction-nature of our cricket-inspired sensory system we have demonstrated that the flow sensitivity can be enhanced by more than $80 \%$, at the cost of a slightly reduced bandwidth (Droogendijk et al., 2012). Similarly, non-resonant parametric amplification has been implemented to improve the responsivity to incoming airflow by up to $20 \mathrm{~dB}$ while having large selectivity with respect to non frequency-matched signals (Droogendijk et al., 2011).

Here, we exploit parametric effects to implement electro mechanical amplitude modulation (EMAM) in order to improve the flow sensitivity at low frequencies. Previously, Raskin et al. (2000) fabricated a MEMS-device as part of an electrical circuit, in which they exploited parametric amplification by electromechanical frequency-upconversion of the electrical input signal. Trusov and Shkel (2007) applied the technique of electromechanical amplitude modulation in a micromachined gyroscope, in order to separate the motional signal from the parasitic feed-

This chapter is based on "Uncovering signals from measurement noise by electro mechanical amplitude modulation" by H. Droogendijk, R. G. P. Sanders, and G. J. M. Krijnen, published in New J. Phys., 15: 053029, (2013). 
through of the applied drive voltages. However, they achieved their upconversion by electrostatically driving their device rather than exploiting parametric mixing of frequencies. Here, we demonstrate EMAM by upconversion of low-frequency airflow signals to higher frequencies using parametric frequency mixing, achieving improved sensor detection thresholds.

The organization of this chapter is as follows. Section 5.2 starts with a theoretical model of the hair flow sensor, followed by the principle of stiffness control using electrostatic forces. Then, the concept of EMAM is introduced, where analytical modelling by harmonic balancing is compared to numerical simulations. Experiments for both harmonic and pulse-like flows are described in section 5.3. In section 5.4, several aspects of EMAM are discussed, like the benefits and its applicability to other domains.

\subsection{Theory and modelling}

A simple picture of the EMAM scheme discussed in this chapter is the following. If in a mechanical system a spring-stiffness can be modulated as a function of time, this will result in temporal variations in response when the spring is subjected to a certain loading. For example, with a constant load its response will vary in time according to the spring modulation. Obviously, without loading the response will be zero. Hence, the response to the combined effects of loading and spring-stiffness modulation will consist of the product of loading times the spring-stiffness variation and therefore the response to the loading has a frequencyshifted component.

To illustrate the principle of EMAM, consider the frequency spectrum shown in figure 5.1. Here, a signal with information containing frequencies between 0 and $\omega_{\mathrm{a}}$ is present. By harmonically modulating the system's torsional stiffness, the information is upconverted to frequencies around the stiffness modulation frequency $2 \omega_{\mathrm{p}}$. As a result, the flow information is shifted to frequencies between $2 \omega_{\mathrm{p}} \pm \omega_{\mathrm{a}}$. Furthermore, the transfer of a mechanical second order system with resonance frequency $\omega_{\mathrm{r}}$ is shown by a red line. Figure 5.1 indicates that the upconverted frequencies should not substantially exceed the system's resonance frequency in order to remain a faithful representation of the original information. Generally, EMAM can be used to shift signal-responses to frequencies that are favourable with respect to the signal to noise ratio. In the following we will give the theoretical basis for the application of this scheme to our rotational hair-based flow-sensors. 


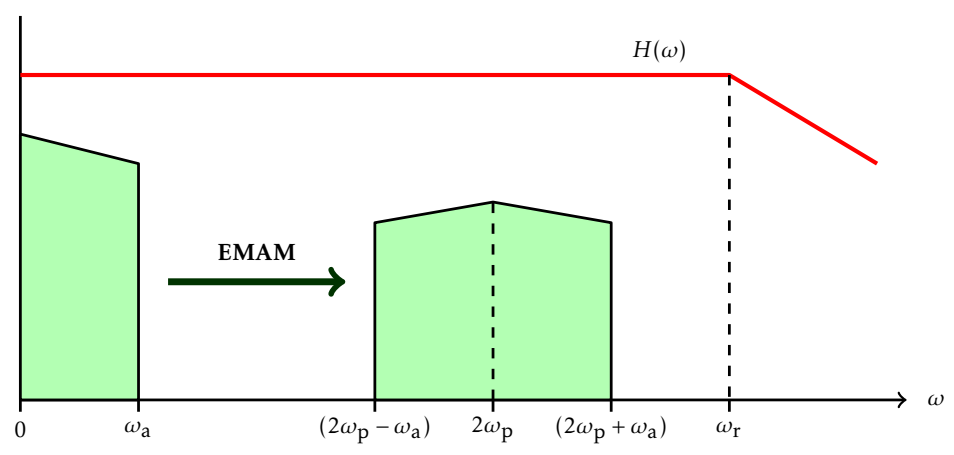

Figure 5.1: Illustration of the principle of EMAM.

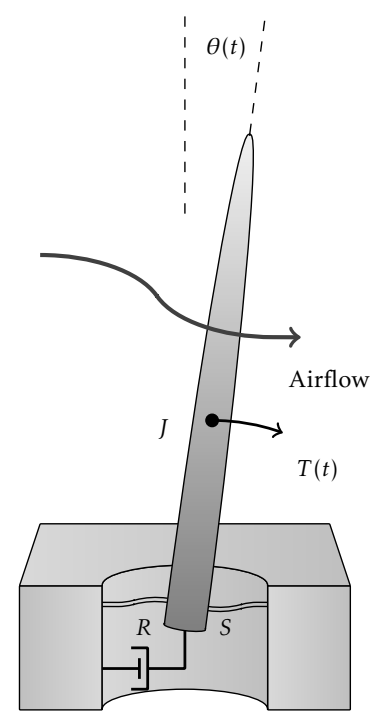

Figure 5.2: Model of a flow sensing hair based on an inverted pendulum (Shimozawa et al., 1998).

\subsubsection{Hair mechanics}

Mechanically, the hair flow sensor can be understood as a so-called inverted pendulum (figure 5.2); a second-order rotational-mechanical system with moment of inertia $J$ due to the hair, a rotational stiffness $S$ and a rotational damping $R$. The airflow generates a drag-torque $T(t)$ on the hair-shaft, mostly by viscous drag (Stokes, 1851) at the velocities and geometries normally encountered, resulting in a description of the system's response by: 


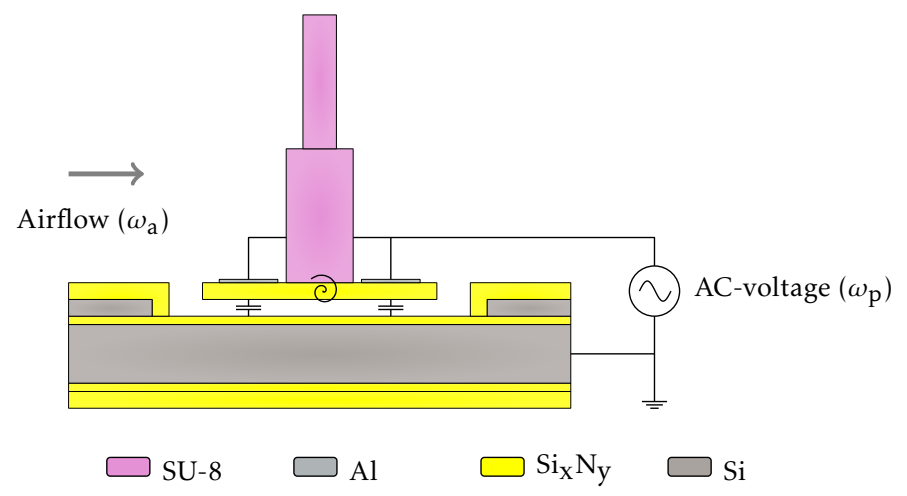

Figure 5.3: Controlling the torsional stiffness by applying ac-bias voltages to the sensor capacitances.

$$
J \frac{\mathrm{d}^{2} \theta(t)}{\mathrm{d} t^{2}}+R \frac{\mathrm{d} \theta(t)}{\mathrm{d} t}+S(t) \theta(t)=T_{0} \cos \left(\omega_{\mathrm{a}} t\right) .
$$

In fact $R$ and $J$ in (5.1) contain added contributions from the hair movement relative to the air which we do not discuss here (see e.g. (Humphrey et al., 1993)). To exploit parametric effects in the hair-based flow sensor, the torsional stiffness $S(t)$ in $(5.1)$ can be controlled by a time-dependent voltage.

\subsubsection{Stiffness modulation}

The electrostatic transduction nature of the system is used to induce balanced electrostatic forces by voltages symmetrically applied to the electrodes (figure 5.3). The resulting torque and stiffness can be calculated from the capacitor geometry. The capacitor is treated as a parallel plate capacitor (figure 5.4) with width $w$, total length $2 L$ and mutual plate distance $g$. The sensor operates in air, for which the relative electric permittivity $\epsilon_{\mathrm{r}}$ is assumed to be equal to 1 . Additionally, the silicon-nitride layers increase the effective dielectric distance to the gap given by $t_{\mathrm{SiN}} / \epsilon_{\mathrm{r}, \mathrm{SiN}}$, leading to an effective gap $g_{\text {eff }}$ :

$$
g_{\text {eff }}=\frac{t_{\mathrm{SiN}, \mathrm{top}}}{\epsilon_{\mathrm{r}, \mathrm{SiN}}}+g+\frac{t_{\mathrm{SiN}, \text { bottom }}}{\epsilon_{\mathrm{r}, \mathrm{SiN}}} .
$$

The angle dependent capacitance $C$ for the rotational sensor is given by:

$$
C=\int_{-L}^{L} \frac{\epsilon_{0} w \cos (\theta)}{g_{\text {eff }}-x \sin (\theta)} \mathrm{d} x
$$




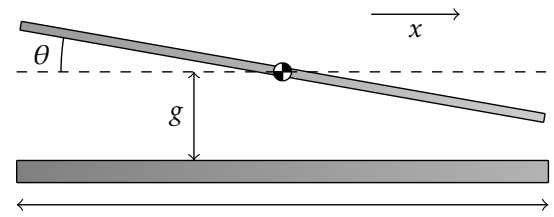

$2 L$

Figure 5.4: Geometry of the angle-dependent rectangular capacitor.

where $x$ is the direction parallel to the plates and $\theta$ is the angle of rotation of the upper plate. Transduction principles are used to find the electrostatic spring softening by an angle-dependent and voltage-controlled capacitor. Applying an ac-bias voltage with amplitude $U_{\mathrm{p}}$, angular frequency $\omega_{\mathrm{p}}$ and phase $\phi_{\mathrm{p}}$ yields the total torsional stiffness $S(t)$ :

$$
S(t)=S_{0}-\frac{1}{4} U_{\mathrm{p}}^{2} \frac{\partial^{2} C}{\partial \theta^{2}}-\frac{1}{4} U_{\mathrm{p}}^{2} \cos \left(2 \omega_{\mathrm{p}} t+2 \phi_{\mathrm{p}}\right) \frac{\partial^{2} C}{\partial \theta^{2}} .
$$

This result shows that the torsional stiffness $S$ is dependent on the parameters of the applied ac-bias voltage, further referred to as the pump voltage $U_{\mathrm{p}}$. The expression states that, under the small rotational angles normally encountered, the total torsional stiffness contains the intrinsic material-based stiffness $S_{0}$, a time-independent softening term and a frequency and phase-dependent softening term.

In the above analysis we have identified a convenient method to produce temporal modulations of the spring-constant in our hair-sensor system. These temporal spring modulations will drive the signals at higher frequencies, as will be derived in the next subsection.

\subsubsection{Electromechanical amplitude modulation}

To achieve electromechanical amplitude modulation (EMAM) in our hair flow sensory system, an airflow with frequency $\omega_{\mathrm{a}}$ together with the pump set at a much higher frequency $\omega_{\mathrm{p}}$ is required. As a consequence of parametric frequency mixing - the interaction between flow response and pump induced springstiffness variations at twice the pump frequency - the flow information will not only be present at the frequency $\omega_{\mathrm{a}}$, but also at the frequencies $2 \omega_{\mathrm{p}} \pm \omega_{\mathrm{a}}$ (see also figure 5.1).

To find an analytical approximation for the amplitude of the frequency components at which the flow information is present after this upconversion, the method of harmonic balancing (Harish et al., 2009) is used. By balancing terms 
containing $\pm \omega_{\mathrm{a}}$ and $\pm\left(2 \omega_{\mathrm{p}} \pm \omega_{\mathrm{a}}\right)$, and truncating the series for higher order terms, the rotational angle amplitudes for the retained frequencies are found.

Consider the second order differential equation from (5.1) describing the hair sensor's mechanics. For convenience, the airflow induced torque $T(t)$ and timedependent torsional stiffness $S(t)$ can be expressed as:

$$
T(t)=\frac{T_{0}}{2}\left[e^{j \omega_{\mathrm{a}} t}+e^{-j \omega_{\mathrm{a}} t}\right], \quad \text { and } \quad S(t)=S_{0}-\frac{1}{2} \frac{\partial^{2} C}{\partial \theta^{2}} u(t)^{2} .
$$

The ac-bias voltage is a harmonic voltage with amplitude $U_{\mathrm{p}}$, phase $\phi_{\mathrm{p}}$ and frequency $\omega_{\mathrm{p}}$ :

$$
u(t)=\frac{U_{\mathrm{p}}}{2}\left[e^{j\left(\omega_{\mathrm{p}} t+\phi_{\mathrm{p}}\right)}+e^{-j\left(\omega_{\mathrm{p}} t+\phi_{\mathrm{p}}\right)}\right]
$$

Squaring the expression for $u(t)$ :

$$
u(t)^{2}=\frac{U_{\mathrm{p}}^{2}}{2}+\frac{U_{\mathrm{p}}^{2}}{4} e^{j 2 \phi_{\mathrm{p}}} e^{j 2 \omega_{\mathrm{p}} t}+\frac{U_{\mathrm{p}}^{2}}{4} e^{-j 2 \phi_{\mathrm{p}}} e^{-j 2 \omega_{\mathrm{p}} t} .
$$

As a result, the torsional stiffness $S(t)$ can be expressed as:

$$
S(t)=S_{0}-\frac{1}{2} \eta U_{\mathrm{p}}^{2}-\frac{1}{4} \eta U_{\mathrm{p}}^{2} e^{j 2 \omega_{\mathrm{p}} t}-\frac{1}{4} \eta U_{\mathrm{p}}^{2} e^{-j 2 \omega_{\mathrm{p}} t},
$$

in which the phase-dependency is suppressed $\left(\omega_{\mathrm{a}} \neq \omega_{\mathrm{p}}\right)$, and $\eta$ represents a geometrical constant:

$$
\eta=\frac{1}{2} \frac{\partial^{2} C}{\partial \theta^{2}} \approx \frac{2 \epsilon_{0} w L^{3}}{3 g_{\mathrm{eff}}^{3}} .
$$

This result shows that the net torsional stiffness $S(t)$ contains four terms: two that are frequency-independent, one at frequency $2 \omega_{\mathrm{p}}$ and one at frequency $-2 \omega_{\mathrm{p}}$.

Since the driving torque is at $\pm \omega_{\mathrm{a}}, \theta(t)$ will contain these frequencies. Due to the product of $S(t)$, given by (5.7), and $u(t)^{2}$, given by (5.6), the rotational angle amplitude $\theta(t)$ will also contain frequency components at $2 \omega_{p} \pm \omega_{a}$ :

$$
\begin{aligned}
\theta(t)= & \theta_{1} e^{j \omega_{\mathrm{a}} t}+\theta_{2} e^{-j \omega_{\mathrm{a}} t}+\theta_{3} e^{j\left(2 \omega_{\mathrm{p}}-\omega_{\mathrm{a}}\right) t}+\theta_{4} e^{-j\left(2 \omega_{\mathrm{p}}-\omega_{\mathrm{a}}\right) t}+ \\
& \theta_{5} e^{j\left(2 \omega_{\mathrm{p}}+\omega_{\mathrm{a}}\right) t}+\theta_{6} e^{-j\left(2 \omega_{\mathrm{p}}+\omega_{\mathrm{a}}\right) t},
\end{aligned}
$$

where higher frequencies are neglected. This system of equations can be approximated as a linear system of coupled differential equations, one for each of the frequencies in (5.9). The differential equations are given by: 


$$
-\omega_{i}^{2} J \theta_{i}+j \omega_{i} R \theta_{i}+[S(t) \theta(t)]_{\omega_{i}}=\frac{T_{0}}{2}\left[\delta\left(\omega_{i}-\omega_{\mathrm{a}}\right) e^{j \omega_{\mathrm{a}} t}+\delta\left(\omega_{i}+\omega_{\mathrm{a}}\right) e^{-j \omega_{\mathrm{a}} t}\right] .
$$

$[S(t) \theta(t)]_{\omega_{i}}$ implies the terms at frequency $\omega_{i}$. The Kronecker delta function $(\delta)$ restricts the driving to the frequency of the airflow $\left(\omega_{\mathrm{a}}\right)$. For convenience, we introduce the shorthand notations:

$$
a=\frac{1}{4} \eta U_{\mathrm{p}}^{2}, \quad b=S_{0}-2 a, \quad c=\frac{T_{0}}{2} .
$$

Furthermore, we define the frequencies:

$$
\begin{array}{lll}
\omega_{1}=\omega_{\mathrm{a}}, & \omega_{3}=2 \omega_{\mathrm{p}}-\omega_{\mathrm{a}}, & \omega_{5}=2 \omega_{\mathrm{p}}+\omega_{\mathrm{a}}, \\
\omega_{2}=-\omega_{1}, & \omega_{4}=-\omega_{3}, & \omega_{6}=-\omega_{5} .
\end{array}
$$

Additionally, we define for the frequency-dependent terms involving $J$ and $R$ :

$$
g(\omega)=-\omega^{2} J+j \omega R, \quad g_{i}=g\left(\omega_{i}\right)
$$

On collecting terms at equal frequencies and neglecting frequencies higher than $2 \omega_{\mathrm{p}}+\omega_{\mathrm{a}}$, we find:

$$
\begin{aligned}
& @ \omega_{1}: g_{1} \theta_{1}+b \theta_{1}-a \theta_{4}-a \theta_{5}=c, \\
& @ \omega_{2}: g_{2} \theta_{2}+b \theta_{2}-a \theta_{3}-a \theta_{6}=c, \\
& @ \omega_{3}: g_{3} \theta_{3}+b \theta_{3}-a \theta_{2}=0, \\
& @ \omega_{4}: g_{4} \theta_{4}+b \theta_{4}-a \theta_{1}=0, \\
& @ \omega_{5}: g_{5} \theta_{5}+b \theta_{5}-a \theta_{1}=0, \\
& @ \omega_{6}: g_{6} \theta_{6}+b \theta_{6}-a \theta_{2}=0 .
\end{aligned}
$$

Note that the equations at $\left(\omega_{1}, \omega_{4}, \omega_{5}\right)$ are decoupled from those at $\left(\omega_{2}, \omega_{3}, \omega_{6}\right)$. For the amplitude $\theta_{1}$ at the flow frequency $\omega_{1}$ we find:

$$
@ \omega_{1}: \theta_{1}=c \cdot \frac{1}{g_{1}+b-a^{2}\left[\frac{1}{g_{4}+b}+\frac{1}{g_{5}+b}\right]} \text {. }
$$

The resulting amplitude for the frequencies containing the flow information by EMAM:

$$
@ \omega_{4}: \theta_{4}=c \cdot \frac{a}{g_{4}+b} \cdot \frac{1}{g_{1}+b-a^{2}\left[\frac{1}{g_{4}+b}+\frac{1}{g_{5}+b}\right]} \text {, }
$$




$$
@ \omega_{5}: \theta_{5}=c \cdot \frac{a}{g_{5}+b} \cdot \frac{1}{g_{1}+b-a^{2}\left[\frac{1}{g_{4}+b}+\frac{1}{g_{5}+b}\right]} \text {. }
$$

The results for $\omega_{2}, \omega_{3}, \omega_{6}$ are the complex conjugated of the results for $\omega_{1}, \omega_{4}$, $\omega_{5}$ respectively. The derived expressions are explicit expressions with parameters that readily can be calculated using the short hand definitions and the values from table 5.1. It is noteworthy to mention that the amplitudes of $\theta_{1}, \theta_{4}$ and $\theta_{5}$ all depend linearly on $c$, representing the input airflow. Therefore, according to the above analysis it follows that the EMAM concept behaves linearly and is applicable to more complex input signals, like transients. However, the weak dependence on input frequency may cause some distortion of the input signal.

The rotation at $\omega_{4}$ or $\omega_{5}$ divided by the rotation at $\omega_{1}$ can be regarded the gain-factors $G$ at the respective frequencies due to the EMAM approach. They are given by:

$$
G_{1 \rightarrow 4}=\frac{a}{g_{4}+b}, \quad G_{1 \rightarrow 5}=\frac{a}{g_{5}+b} .
$$

In the limit for $U_{\mathrm{p}} \rightarrow 0$, $a$ will be 0 and therefore $\theta_{4}=\theta_{5}=0$ and $\theta_{1}=c /\left(g_{1}+b\right)$ coinciding with the intrinsic mechanical response (i.e. without the use of biasvoltages). Conversely, the norm of the denominators in these expressions may be smaller than $a$ for proper combinations of $U_{\mathrm{p}}$ and $\omega_{i}$ 's, meaning that there can be a genuine gain of the signal. The conditions under which a large gain may occur will in general be close to the conditions under which pull-in (i.e. instability) may take place, therefore requiring careful choice of the pump signal. Still, even at gain values below 1 , there may be a considerable improvement of the signal-to-noise ratio (SNR) in case the noise level at the frequency of the upconverted signal is much lower than the noise level a the frequency of the original signal.

For comparison and validation, the outcome of the analytical results by harmonic balancing are compared to numerical simulations by the Runge-Kutta 4 method from MATLAB ${ }^{\circledR}$. Both results are shown in figure 5.5, using the parameters given in table 5.1. Also the impact of the addition of higher order terms with frequencies $4 \omega_{\mathrm{p}} \pm \omega_{\mathrm{a}}$, following the same analysis by harmonic balancing, is shown. We observe that the simulations and analytical approximations by harmonic balancing are in good agreement. Generally, when the pump amplitude $f_{\mathrm{p}}$ is increased, the rotation angle is increased, at the cost of a slightly reduced bandwidth.

Both simulations and model show that the pump frequency should not be chosen higher than about $500 \mathrm{~Hz}$, since we observe that for higher frequencies 


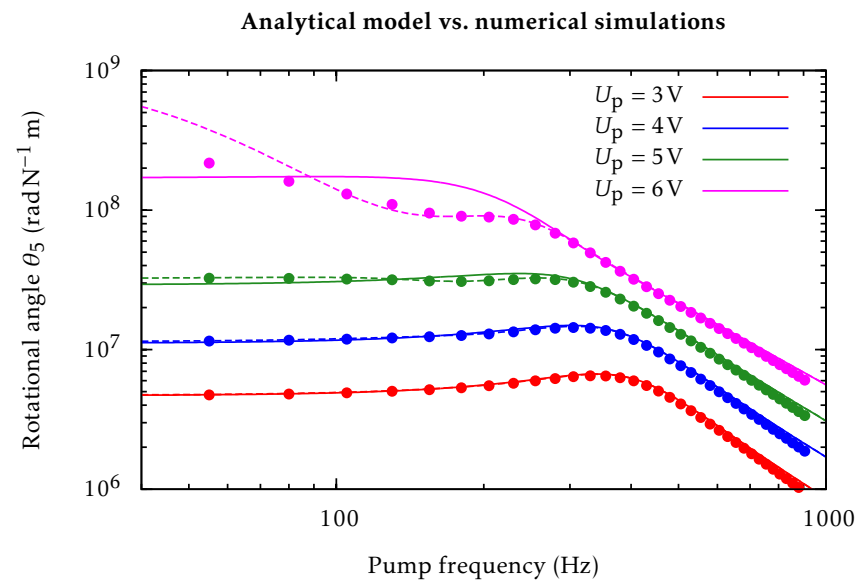

Figure 5.5: Frequency transfer comparison between the analytical model by harmonic balancing using terms $\omega_{\mathrm{a}}$ and $2 \omega_{\mathrm{p}} \pm \omega_{\mathrm{a}}$ (solid lines), harmonic balancing using terms $\omega_{\mathrm{a}}$, $2 \omega_{\mathrm{p}} \pm \omega_{\mathrm{a}}$ and $4 \omega_{\mathrm{p}} \pm \omega_{\mathrm{a}}$ (dashed lines), and the numerical simulations (points) for various pump amplitudes with the flow frequency $f_{\mathrm{a}}$ set to $10 \mathrm{~Hz}$.

the rotational angle $\theta_{5}$ rapidly decreases. Further, due to the quadratic nature of electrostatic forces, a pump frequency of $500 \mathrm{~Hz}$ coincides with a mechanical frequency of $1000 \mathrm{~Hz}$, which is just slightly above the sensor's resonance frequency (about $800 \mathrm{~Hz}$ ).

To gain some more insight we may simplify the derived full expressions for $\omega_{1}, \omega_{4}$ and $\omega_{5}$, using the approximation that the upconverted flow information is at frequencies still sufficiently below the sensor's resonance frequency, i.e. by letting $g_{i} \rightarrow 0$. As a result, we find:

$$
\begin{gathered}
@ \omega_{1}: \theta_{1}=c \cdot \frac{b}{b^{2}-2 a^{2}}, \\
@ \omega_{4,5}: \theta_{4,5}=c \cdot \frac{a}{b^{2}-2 a^{2}} .
\end{gathered}
$$

By using the same approach for the frequencies $\omega_{2}, \omega_{3}, \omega_{6}$ and by resubstituting the various physical constants, we find for the rotational amplitudes:

$$
\theta_{1,2}=\frac{T_{0}}{S_{0}} \frac{\left(1-\lambda U_{\mathrm{p}}^{2}\right)}{\left(\lambda U_{\mathrm{p}}^{2}\right)^{2}-4 \lambda U_{\mathrm{p}}^{2}+2}, \quad \text { with } \quad \lambda=\frac{\eta}{2 S_{0}}
$$


Table 5.1: Parameter values of the hair mechanical system and the capacitor.

\begin{tabular}{lll}
\hline Quantity & Symbol & Value \\
\hline Mechanical parameters & & \\
\hline Torsional stiffness & $S_{0}$ & $9.5 \times 10^{-9} \mathrm{Nm} \mathrm{rad}^{-1}$ \\
Torsional resistance & $R$ & $1.2 \times 10^{-12} \mathrm{Nm} \mathrm{sad}^{-1}$ \\
Moment of inertia & $J$ & $3.1 \times 10^{-16} \mathrm{~kg} \mathrm{~m}^{2}$ \\
\hline Sensor specifications & & \\
\hline Quality factor & $Q$ & 1.43 \\
Resonance frequency & $f_{0}$ & $881 \mathrm{~Hz}$ \\
\hline Hair geometry & & \\
\hline Hair length & $L_{\mathrm{h}}$ & $800 \mu \mathrm{m}$ \\
Hair diameter & $d_{\mathrm{h}}$ & $50 \mu \mathrm{m}$ \\
\hline Capacitive structures & & \\
\hline Membrane length & $L$ & $95 \mu \mathrm{m}$ \\
Membrane width & $w$ & $90 \mu \mathrm{m}$ \\
Gap distance & $g$ & $850 \mathrm{~nm}$ \\
Nitride thickness & $t_{1}$ & $1000 \mathrm{~nm}$ \\
Nitride thickness & $t_{2}$ & $200 \mathrm{~nm}$ \\
Relative permittivity & $\epsilon_{\mathrm{r}}$ & 7.5 \\
\hline
\end{tabular}

$$
\theta_{3,4,5,6}=\frac{T_{0}}{2 S_{0}} \frac{\lambda U_{\mathrm{p}}^{2}}{\left(\lambda U_{\mathrm{p}}^{2}\right)^{2}-4 \lambda U_{\mathrm{p}}^{2}+2}=\frac{\theta_{1,2}}{2} \frac{\lambda U_{\mathrm{p}}^{2}}{\left(1-\lambda U_{\mathrm{p}}^{2}\right)}
$$

The geometrical and other properties of the capacitive structure, as well as the parameters of the hair flow sensory system, as used in the calculation of the theoretical response are listed in table 5.1.

Our numerical and analytical analyses indicate that we can use harmonic spring-stiffness variations to generate replica of the incoming flow signals at higher frequencies. In the next section we will describe the experimental findings and identify the benefits of the EMAM scheme. 


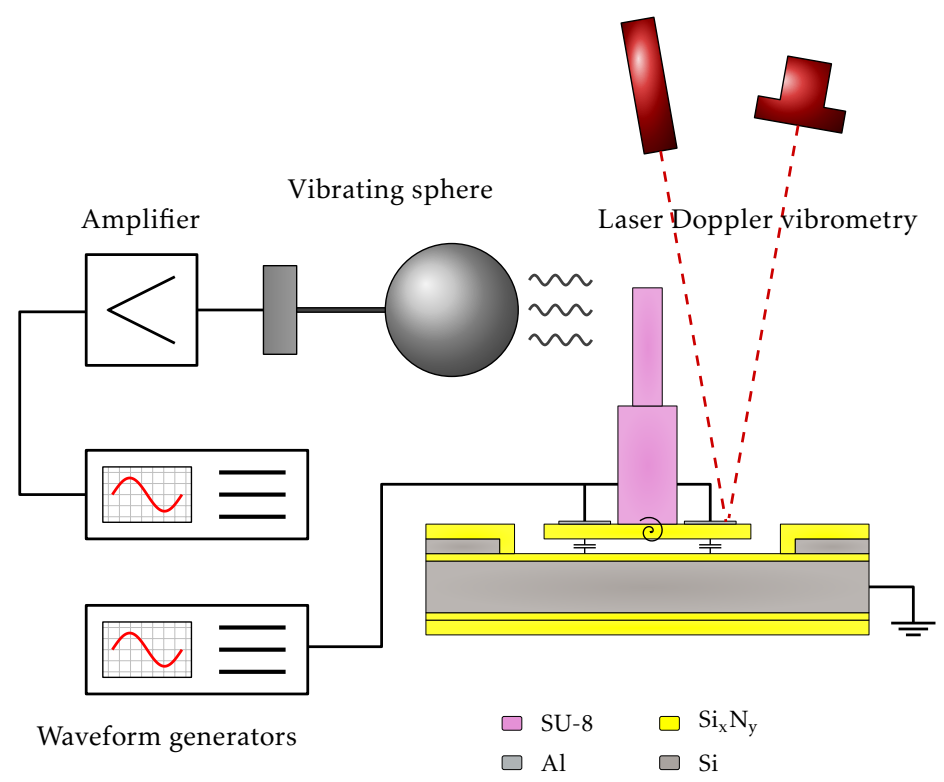

Figure 5.6: Experimental setup for measuring membrane movements using laser Doppler vibrometry.

\subsection{Experimental}

\subsubsection{Setup}

Experiments are performed using the setup shown in figure 5.6. A waveform generator (Agilent 33220A-001) is used to generate a sinusoidal signal at a frequency $f_{\mathrm{a}}$ that is applied to an amplifier. This amplifier drives a vibrating sphere to generate the oscillating airflow field described by a dipole source (Lamb, 1932). A second waveform generator (Agilent 33220A-001) is used to supply the ac-pump voltage to the top electrodes. The bottom electrode is grounded as is the measurement setup. The sensor rotational angular velocity $\theta$ is derived from laser Doppler vibrometry using a Polytec MSA-400, since there is a simple factor between the measured membrane velocity and the hair rotational angular velocity $\dot{\theta}$. The resulting vibrometer output voltage representing $\dot{\theta}$ is fed to an adjustable band pass filter (Stanford SR 650). To demodulate the membrane velocity by synchronous detection the filtered output is supplied to a lock-in amplifier (Stanford SR 830) with its reference frequency set to twice the pump frequency. 


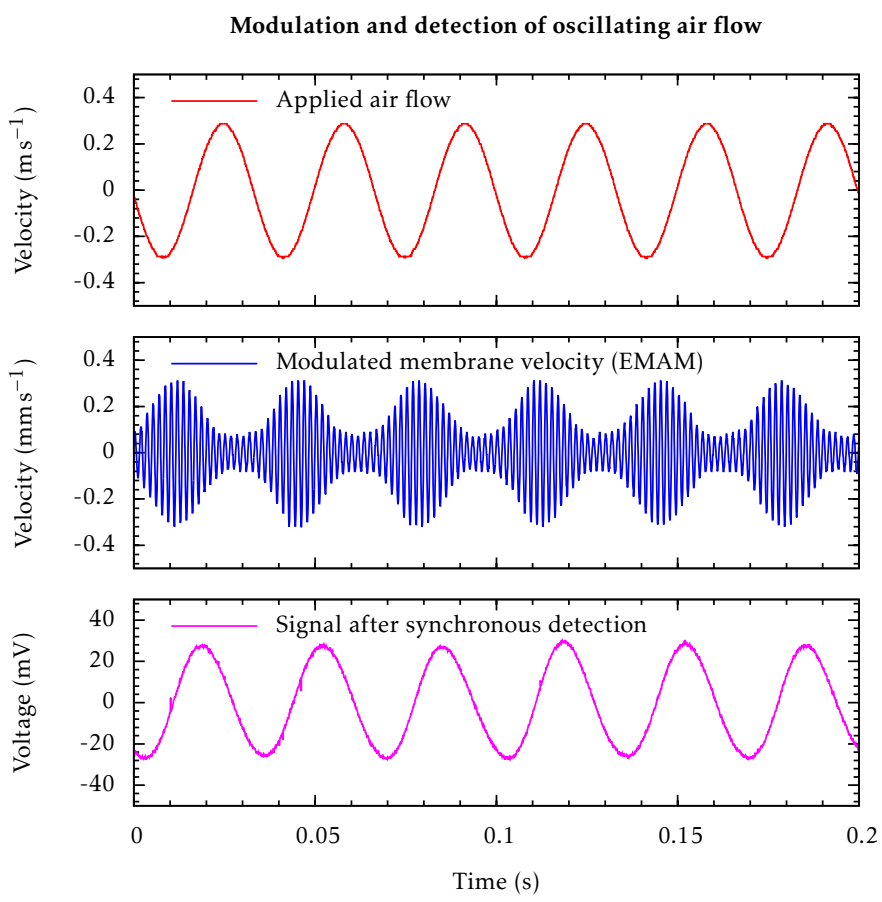

Figure 5.7: The applied periodic airflow $(30 \mathrm{~Hz})$ using a vibrating sphere, the amplitude modulated membrane velocity and the synchronous detected flow signal $(30 \mathrm{~Hz})$ in the time-domain. The ac-bias voltage was set to $300 \mathrm{~Hz}$ with an amplitude of $3 \mathrm{~V}$.

\subsubsection{Demonstration of EMAM}

To demonstrate EMAM in our biomimetic hair flow sensors, the vibrating sphere was used to generate a periodic flow at $30 \mathrm{~Hz}$. The amplitude of the ac-bias voltage was set to $3 \mathrm{~V}$ and its frequency to $300 \mathrm{~Hz}$. As a result, by the quadratic nature of electrostatic actuation, the torsional stiffness is modulated at twice the frequency, $600 \mathrm{~Hz}$. Figure 5.7 shows the measured (modulated) membrane velocity, together with the voltage applied to the vibrating sphere and the resulting signal after synchronous detection (at $600 \mathrm{~Hz}$ ) using the lock-in amplifier.

We observe that with the use of EMAM the flow signal at $30 \mathrm{~Hz}$ produces an acmodulated membrane rotation around $600 \mathrm{~Hz}$ (middle graph) largely reflecting the low frequency signal in the amplitude. There is an additional contribution from the vertical vibrations of the sensor's membrane at $600 \mathrm{~Hz}$ but this component is suppressed in the demodulated membrane rotational velocity (lower graph), which clearly represents the incoming airflow. 


\subsubsection{Improving measurement quality}

The output rms-voltage of the vibrometer signal was measured for varying flow velocities (figure 5.8), still at a frequency of $30 \mathrm{~Hz}$, both for the original flow signal (band-pass filter set to $25-35 \mathrm{~Hz}$ ) and the demodulated EMAM signal (band-pass filter set to $565-635 \mathrm{~Hz}$ ). The ac-bias voltage, at $300 \mathrm{~Hz}$, was varied in amplitude from $3 \mathrm{~V}$ to $6 \mathrm{~V}$. The quality of the measurements at lower frequencies is significantly improved using EMAM. Without EMAM, the measured output voltage as a function of flow amplitude (see figure 5.8 lowest curve) is rather noisy. With EMAM a clear linear relationship is observed for flow velocity amplitudes above threshold.

We use the results of figure 5.8 to determine the lowest detectable (threshold) flow-amplitudes as a function of $U_{\mathrm{p}}$. The output voltage is assumed to be proportional to the flow velocity amplitude $V_{0}$, given by a coefficient $S_{\mathrm{c}}\left(U_{\mathrm{p}}\right)$. Letting $N_{\mathrm{sc}}$ be a constant representing the rms-value of the additive, uncorrelated, noise power, the measured output voltage $U_{\text {out }}$ can be represented as (Droogendijk et al., 2012):

$$
U_{\text {out }}=\sqrt{\left(S_{\mathrm{c}} V_{0}\right)^{2}+N_{\mathrm{sc}}^{2}}
$$

We define the flow threshold as the intersection of the two asymptotes of (5.23), given by $\mathrm{SNR}=1$, which is $N_{\mathrm{sc}} / S_{\mathrm{c}}$. The solid curves in figure 5.8 represent fits to the measurements according to (5.23) and yield the threshold flow-amplitudes.

Although a high pump amplitude $U_{\mathrm{p}}$ potentially provides a significant improvement in flow sensitivity, the sensor response deteriorates for higher flow velocity amplitudes. The combination of finite vertical stiffness of the torsion beams, a strong pump and a large airflow results in displacements of the membrane in the order of tens of percent of the gap distance $g$. As a consequence, these effects contribute to the system's response, resulting in a non-linear relationship between output rms-voltage and driving airflow (orange circles in figure 5.8).

Approximating the threshold velocity to be inversely proportional with the rotational angle, using (5.22), the comparison between model and measurements is made in figure 5.9. It demonstrates that (5.22) can be used well to describe the improvement in threshold reduction as a function of pump voltage $U_{\mathrm{p}}$. We observe that increasing $U_{\mathrm{p}}$ will cause the detection limit to decrease non-linearly, but may also affect the stability of the system for high values of $U_{\mathrm{p}}$. When $U_{\mathrm{p}}$ reaches a critical value, the total torsional stiffness becomes zero causing pull-in instability, which is also predicted by the analytical model.

The measurements show that EMAM in the studied case reduces the detection limit by a factor of 25 (from $50 \mathrm{~mm} \mathrm{~s}^{-1}$ to $2 \mathrm{~mm} \mathrm{~s}^{-1}$ ). At the same time it is obvious 


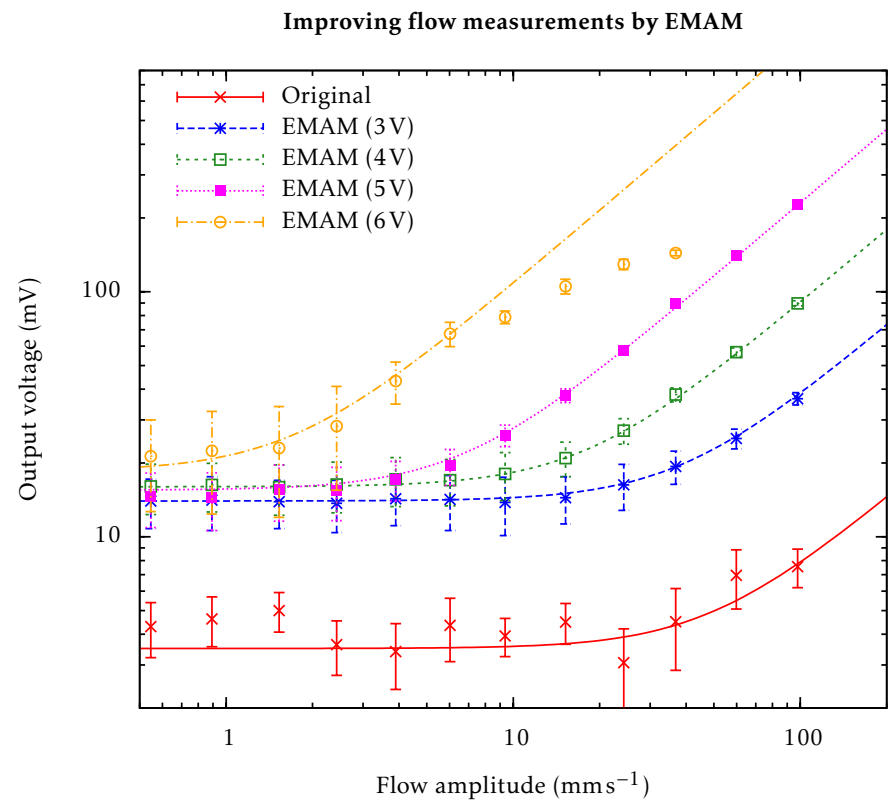

Figure 5.8: Improvement of the quality of the measured rms-voltage values at low frequency signals using EMAM. In case of EMAM, a clear linear relationship between flow and output voltage is observed above the system's noise level. The symbols represent experimental data with the measurement error indicated, and the solid lines show the fitted expression for the output voltage based upon (5.23).

that improvement of the detection limit requires a certain minimum value for $U_{p}$, which is about $3 \mathrm{~V}$ in our case (intersection of plots in figure 5.9).

\subsubsection{Application of EMAM to transient flows}

Although the application of sinusoidal airflows simplifies modelling and enables quantitative investigation of EMAM, the use of transient signals with increased spatio-temporal flow information, is more akin to what one may want to measure in practice than sinusoidal flows. In nature, there are numerous examples representing transient airflow stimuli such as spider motion (Dangles et al., 2006) and (passing) humming flies (Barth et al., 1995). Furthermore, such stimuli appear to have most of their signal power present in the lower frequency range, emphasizing the requirement to have sufficient sensor responsivity at low frequencies (Kant and Humphrey, 2009). Therefore, we also performed measurements on pulsed-like airflows. 


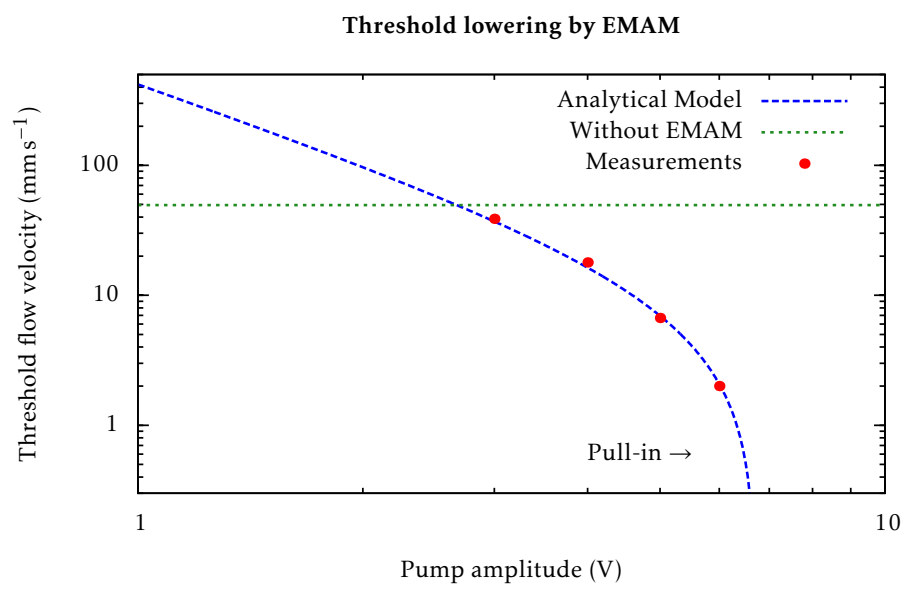

Figure 5.9: Reduction of threshold flow-amplitude versus pump amplitude $U_{p}$. The maximum reduction obtained is 25 times.

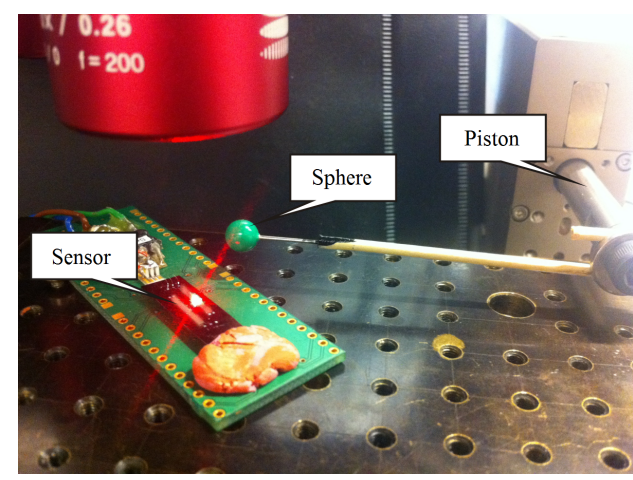

Figure 5.10: Transient measurement set-up using a moving sphere as flow source.

Previously, we measured responses of our biomimetic hair flow sensors to airflow transients using a sphere with $3 \mathrm{~mm}$ radius attached to a piston system to represent the motion of a spider at a certain distance from the sensor (Krijnen et al., 2013). Now, we use a similar sphere with $3 \mathrm{~mm}$ radius (figure 5.10) mounted on a moving piston (SMAC LCA25-050-15F). As an experimental setup, the previously described setup for optical investigation of EMAM is used (figure 4.2).

The tiny sphere was used to generate a transient airflow, where the piston speed was set to $20 \mathrm{~cm} \mathrm{~s}^{-1}$. The amplitude of the ac-bias voltage was set to $4 \mathrm{~V}$ and its frequency to $400 \mathrm{~Hz}$. As a result, by the quadratic nature of electrostatic actuation, the torsional stiffness is modulated at twice the frequency, $800 \mathrm{~Hz}$. The 


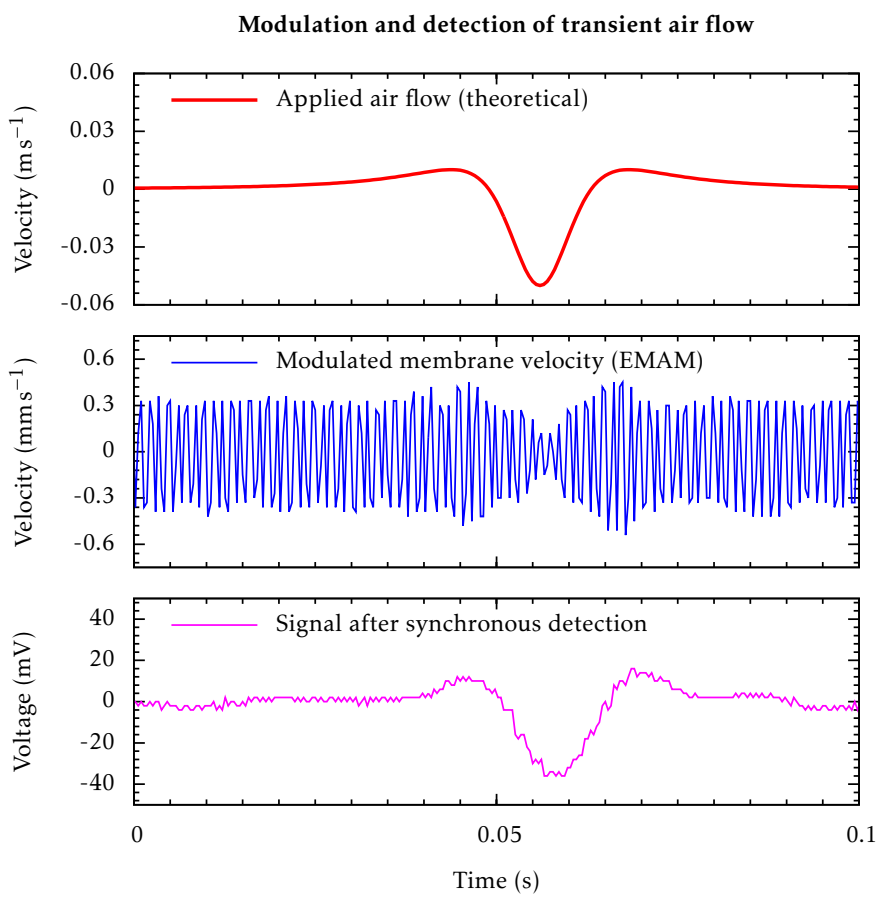

Figure 5.11: The amplitude modulated membrane velocity and the synchronous detected flow signal in the time-domain of the transient airflow. The ac-bias voltage was set to $400 \mathrm{~Hz}$ with an amplitude of $4 \mathrm{~V}$.

filter's bandwidth was adjusted to $760-840 \mathrm{~Hz}$. Furthermore, the time constant of the lock-in amplifier was set to $10 \mathrm{~ms}$ and its low pass filter to $24 \mathrm{~dB} /$ oct roll-off.

Figure 5.11 shows in succession the theoretical applied airflow according to Lamb (1932), the measured (modulated) membrane velocity, and the resulting signal after synchronous detection $($ at $800 \mathrm{~Hz})$ using the lock-in amplifier. We observe that with the use of EMAM the transient airflow signal produces an acmodulated membrane rotation. The demodulated membrane rotational velocity clearly represents the incoming airflow, proving that EMAM can also be used to perform measurements on more complex signals. In addition, without the application of EMAM, we were not able to obtain a representation of the applied pulsed-like airflow at all, emphasizing the advantage of EMAM under the given experimental conditions.

To explain the improvement in signal-to-noise ratio (SNR) while using EMAM, the spectrum of the resolved transient flow (figure 5.11) is shown in figure 5.12 (red solid line). We observe that the overall spectrum is in good agreement with the theoretical analysis of Kant and Humphrey (2009) for pulsed airflows, since 


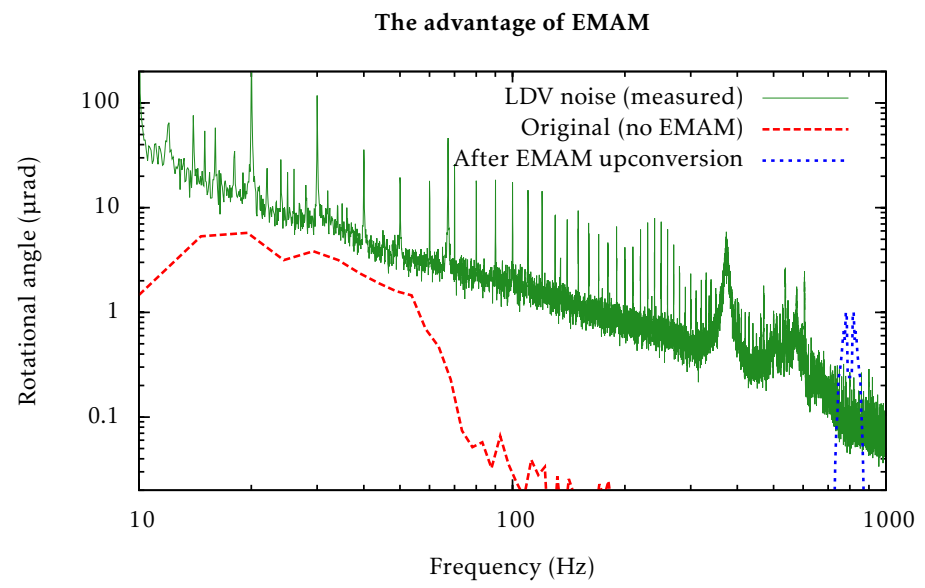

Figure 5.12: Illustration of the advantage of EMAM for low-frequency flows. Originally, the signal spectral density is well below the noise floor of the measurement setup. On application of EMAM with a pump frequency of $400 \mathrm{~Hz}$, the transient flow spectrum is upconverted to frequencies around $800 \mathrm{~Hz}$. At these frequencies the signal-to-noise ratio of the transient flow is clearly larger than one, enabling detection of the pulsed airflow.

most of the signal power is found in frequencies within a range of about $10-60 \mathrm{~Hz}$.

Furthermore, the noise level of the measurement setup (green solid line) is higher than the flow-induced sensor response, thus entirely masking the airflow. However, in case of EMAM the flow-information is frequency upconverted (blue solid line) to frequencies around $800 \mathrm{~Hz}$. Although the rotational angle amplitudes are lower than compared to the case without EMAM, we observe also that the SNR at these higher frequencies is clearly larger than one. Therefore, by applying EMAM it is possible to measure low-frequency airflows which are normally covered in noise.

Clearly, the experimental data supports the notion that application of EMAM can be beneficially used to improve signal-to-noise ratios of harmonic signals and even uncover transient signals from measurement noise. In the next section we will discuss the conditions under which these improvements can be obtained and the applicability to other sensor systems. 


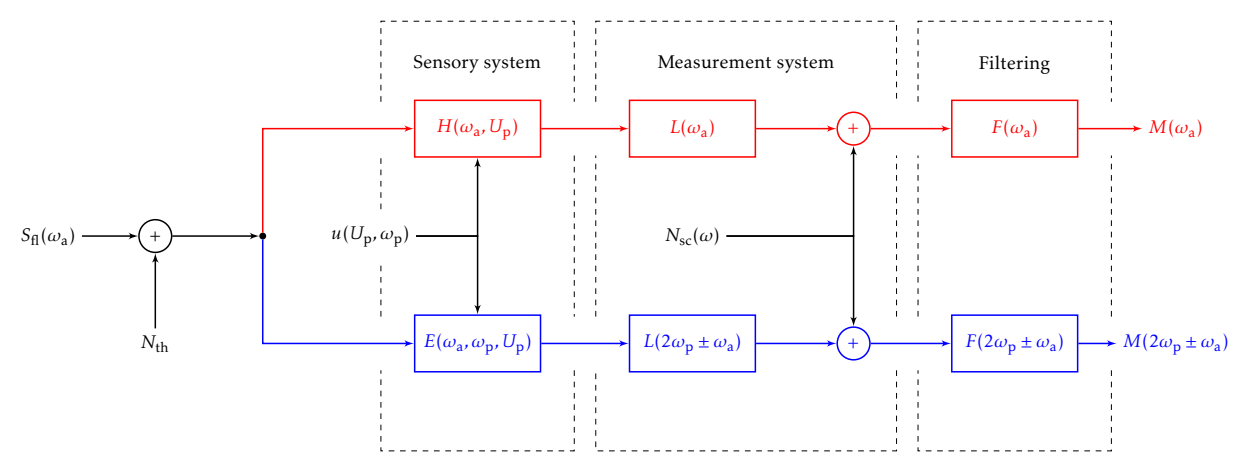

Figure 5.13: Schematic view of the system for identification of the system's noise sources. The upper path (red) illustrates the case without EMAM, the lower path (blue) shows the impact of EMAM.

\subsection{Discussion}

\subsubsection{Noise analysis}

To explain why electromechanical amplitude modulation (EMAM) can be used to enhance the measurable flow velocity range and/or the quality of the measurements, the origin of the noise sources needs to be discussed. A schematic of the system is shown in figure 5.13, which contains the flow signal $S_{\mathrm{fl}}(\omega)$, the sensor's thermal mechanical noise $N_{\text {th }}$, the mechanical transfer of the sensory system $H\left(\omega_{\mathrm{a}}, U_{\mathrm{p}}\right)$, the transfer by EMAM $E\left(\omega_{\mathrm{a}}, \omega_{\mathrm{p}}, U_{\mathrm{p}}\right)$, the applied voltage $u\left(U_{\mathrm{p}}, \omega_{\mathrm{p}}\right)$, the transfer of the measurement system $L(\omega)$, filtering of the measurement signal $F(\omega)$ and other noise sources $N_{\mathrm{sc}}(\omega)$ independent of the sensory system.

In case of dominant non-flow related noise sources $N_{\mathrm{sc}}(\omega)$, with larger power spectral density at $\omega_{a}$ than at $2 \omega_{p} \pm \omega_{a}$ (e.g. in case of $1 / \mathrm{f}$ noise), and/or in case the transfer function $L\left(2 \omega_{p} \pm \omega_{a}\right)$ mediates larger transfer than $L\left(\omega_{a}\right)$, the required airflow to achieve a detectable hair-rotation can be lower at $2 \omega_{p} \pm \omega_{a}$ than at $\omega_{a}$. In these cases EMAM can help to achieve a better low-frequency detection limit by up converting the information to higher frequencies.

To explain our achieved improvement in flow measurement on application of EMAM, the noise spectrum of the experimental setup only (thus without flow) is measured (figure 5.14) and related to the sensor's rotational angle $\theta$. The spectrum exhibits a typical $1 / f$ characteristic, meaning less signal is required at higher frequencies to achieve a suitable signal-to-noise ratio. The spectrum shows peaks at multiples of $10 \mathrm{~Hz}$, which affects flow measurements performed at these frequencies. Also the noise level of the Polytec MSA-400 system, which is specified 


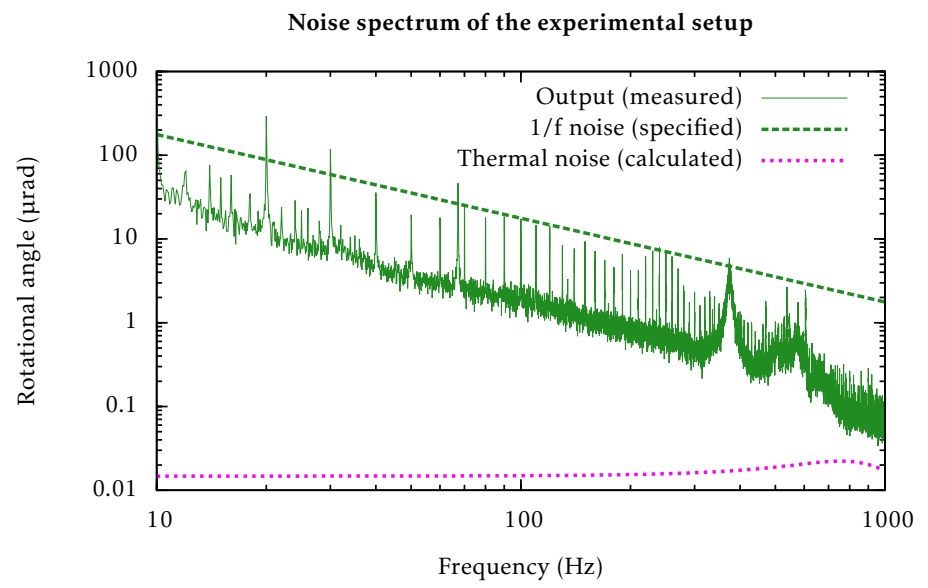

Figure 5.14: Measured spectrum of the experimental setup in absence of airflow.

as $<1 \mu \mathrm{m} \mathrm{s}^{-1}$ (Polytec, 2005), is given in figure 5.14 (green solid line). We observe that the measured output noise level is just below the specified noise level (green dashed line), suggesting that the output signal is dominated by the measurement equipment of the Polytec MSA-400 system.

To compare, the overall noise angle is significantly larger than expected based on thermal mechanical noise calculations (Droogendijk et al., 2012), also shown in figure 5.14 (magenta dotted line). Therefore, the output noise is dominated by non-flow related noise contributions and for this situation EMAM improves the detection limit and measurement quality of low frequency airflows.

\subsubsection{Influence of higher frequency components}

One may question why a relative small number of frequency components as used in the derivation of equations $(5.15,5.16,5.17)$ delivers results so accurately reproducing the EMAM numerical and experimental results. It can be readily shown that the next four frequency terms that can be added to equation (5.14) depend on $a^{2}$. Since normally $a \ll 1$ these higher frequency terms are much smaller and play only a minor role for $\theta_{4}$ and $\theta_{5}$. These amplitudes, which are proportional to $a$ in the derived expressions $(5.15,5.16,5.17)$, now get an additional contribution proportional to $a^{3}$. Additionally, these contributions have denominators $\left[\left(g_{5}+b\right)\left(g_{9}+b\right)-a^{2}\right]$ times larger, resulting in even lower contributions for most values of $\omega_{\mathrm{p}}$ and $a$. Therefore, for all situations, except those close to pull-in instability, the higher frequency terms are of no concern, 
which is in agreement with the comparison between numerical simulations and harmonic balancing in figure 5.5 .

\subsubsection{EMAM with electronic read-out}

In normal operation of our hair-sensors we use differential-capacitive electronic read-out (Bruinink et al., 2009; Dagamseh et al., 2010, 2012), whereas here we have shown the concept of EMAM using optical read-out by vibrometry. The reason we did not use the electronic read-out in our EMAM experiments is that the pump voltages used tend to saturate the electronics, since they are orders of magnitude larger than the tiny signals generated by the capacitive modulation. However, we believe this is no fundamental problem but can be solved by either using electronics with larger dynamic range, or applying a sharp filter at $2 \omega_{\mathrm{p}}$ in the electronics. As far as the improvement by EMAM due to noise in the read-out electronics is concerned, the improvement will depend on the character of the electronics noise. For $1 / f$ type dominated noise, comparable improvement as shown here is expected.

\subsubsection{Extension of the concept}

The theory we have used to derive the expressions for the EMAM amplitudes has been based entirely on the nature of energy buffering transducers. In more detail, an equivalent form of equation (5.4) can be derived for any energy buffering transducer, thus not only electrostatic, but also piezo-electric, electromagnetic and other types of energy buffering transducers. Therefore, we argue that EMAM has a wide application potential, especially with respect to low-frequency sensing. That is, it may for example be beneficial for gravity gradiometry, be applied in seismometers, in highly sensitive accelerometers and other comparable sensors and measurement systems.

\subsection{Conclusions}

Concluding, electromechanical amplitude modulation has been applied to biomimetic hair flow sensors. Using harmonic electrostatic spring softening, by application of an appropriate sinusoidal voltage on the capacitor plates, upconversion of the flow information is achieved. It is demonstrated that EMAM can improve the measurement performance at low frequencies, in case of limitations within the measurement setup. We have shown that the method can be applied equally well to transients as to harmonic signals. In the latter case we have presented 
improvements in threshold limits of up to 25 times, whereas in the transient measurements it was demonstrated that tiny signals, which were below the noiselevels in the base-band measurement, could be revealed well when upconverted to higher frequencies. We have argued that the method is not restricted to our sensors but can be generally applied to energy buffering transducers.

\section{References}

Barth, F. G., Wastl, J. A. H. U., Halbritter, J., and Brittinger, W. (1995). Dynamics of arthropod filiform hairs. III. Flow patterns related to air movement detection in a spider (Cupiennius salei). Phil. Trans. R. Soc. B, 347:397-412.

Bruinink, C. M., Jaganatharaja, R. K., de Boer, M. J., Berenschot, J. W., Kolster, M. L., Lammerink, T. S. J., Wiegerink, R. J., and Krijnen, G. J. M. (2009). Advancements in technology and design of biomimetic flow-sensor arrays. In Proc. MEMS 2009, pp. 152-155.

Dagamseh, A. M. K., Bruinink, C. M., Droogendijk, H., Wiegerink, R. J., Lammerink, T. S. J., and Krijnen, G. J. M. (2010). Engineering of biomimetic hair-flow sensor arrays dedicated to high-resolution flow field measurements. In Proc. IEEE Sensors 2010, pp. 2251-2254, Waikoloa, HI, United States.

Dagamseh, A. M. K., Wiegerink, R. J., Lammerink, T. S. J., and Krijnen, G. J. M. (2012). Towards a high-resolution flow camera using artificial hair sensor arrays for flow pattern observations. Bioinsp. Biomim., 7:046009.

Dangles, O., Ory, N., Steinmann, T., Christides, J. P., and Casas, J. (2006). Spider's attack versus cricket's escape: velocity modes determine success. Anim. Behav., 72:603-610.

Droogendijk, H., Bruinink, C. M., Sanders, R. G. P., Dagamseh, A. M. K., Wiegerink, R. J., and Krijnen, G. J. M. (2012). Improving the performance of biomimetic hair-flow sensors by electrostatic spring softening. J. Micromech. Microeng., 22(6):065026.

Droogendijk, H., Bruinink, C. M., Sanders, R. G. P., and Krijnen, G. J. M. (2011). Non-resonant parametric amplification in biomimetic hair flow sensors: Selective gain and tunable filtering. Appl. Phys. Lett., 99(21):213503.

Harish, K. M., Gallacher, B. J., Burdess, J. S., and Neasham, J. A. (2009). Experimental investigation of parametric and externally forced motion in resonant MEMS sensors. J. Micromech. Microeng., 19:15-21. 
Humphrey, J., Devarakonda, R., Iglesias, I., and Barth, F. (1993). Dynamics of arthropod filiform hairs. I. Mathematical modeling of the hair and air motions. Phil. Trans.: Bio. Sc., 340:423-444.

Kant, R. and Humphrey, J. A. C. (2009). Response of cricket and spider motionsensing hairs to air flow pulsations. J. R. Soc. Interface, 6(6):1047-1064.

Krijnen, G., Droogendijk, H., Dagamseh, A., Jaganatharaja, R., and Casas, J. (2013). Imitating the cricket cercal system: The beauty of the beast with a twist of the engineer. Adv. Sci. Tech., 84:19-28.

Lamb, H. (1932). Hydrodynamics. Cambridge University Press, Cambridge, $6^{\text {th }}$ edition.

Polytec (2005). MSA-400 Micro System Analyzer. Polytec, Waldbronn, Germany.

Raskin, J., Brown, A. R., Khuri-Yakub, B. T., and Rebeiz, G. M. (2000). A novel parametric-effect MEMS amplifier. J. Microelectromech. S., 9:528-537.

Shimozawa, T., Kumagai, T., and Baba, Y. (1998). Structural scaling and functional design of the cercal wind-receptor hairs of cricket. J. Comp. Physiol. A, 183:171186.

Stokes, G. G. (1851). On the effect of the internal friction of fluids on the motion of pendulums. Trans. Cambr. Phil. Soc., 9:1-141.

Trusov, A. A. and Shkel, A. M. (2007). Capacitive detection in resonant MEMS with arbitrary amplitude of motion. J. Micromech. Microeng., 17:1583-1592. 


\section{6 \\ Stochastic resonance in a micromechanical slider}

\subsection{Introduction}

In sensory systems, noise generally limits the sensing performance as a consequence of a finite signal-to-noise ratio (SNR). However, there are conditions under which noise can contribute to an increase in SNR. Levin and Miller (1996) observed that additive noise helps crickets to improve their flow-sensing capabilities, resulting in one of the most sensitive sensing modalities in the animal kingdom, working at levels as low as the thermal-mechanical noise threshold (Shimozawa et al., 2003). Bahar and Moss (2004) found that crayfish use environmental noise for detection of sub-threshold periodic signals. The mechanism to use a tuned amount of noise in combination with sub-threshold signals to increase sensitivity is called Stochastic Resonance - the system shows maximum SNR at a specific amount of noise (Wellens et al., 2004), deteriorating both for more and less noise power.

The principle of stochastic resonance (SR) has been studied for many years within different fields. The idea and impact of SR was introduced by Benzi et al. (1982). They proposed that this mechanism might have a role in the global climatic

This chapter is based on "Stochastic resonance in a voltage-controlled micromechanical slider" by H. Droogendijk, M. J. de Boer, R. G. P. Sanders, and G. J. M. Krijnen, submitted to New J. Phys., (2014). 
system by explaining recurring peaks in the power spectra of paleoclimatic records. Harmer and Abbott (2001) applied the mechanism of SR to motion detectors to improve the sensor performance in noisy environments. Coherent signal amplification in a bistable nanomechanical oscillator by SR was demonstrated by Badzey and Mohanty (2005), with possible applications in e.g. controllable high-speed nanomechanical memory cells. Another nanomechanical application of SR was presented by Almog et al. (2007), by achieving noise-dependent signal amplification in a Duffing resonator. In the optical domain, SR was implemented by Palonpon et al. (1998) to measure weak transmittances. Ono et al. (2008) showed that SR can be used for sensing light and magnetic forces using a nonlinear silicon microresonator. To enhance energy harvesting, SR was applied by McInnes et al. (2008), by adding periodic forcing to a vibrationally excited energy harvesting mechanism enhancing the available power from their device over a mechanism without periodic forcing and only exploiting environmental noise. Li et al. (2013) proposed the application of SR in wind turbines for condition monitoring. Using a a noise-controlled second-order enhanced SR method based on the Morlet wavelet transform, the fault feature for wind turbine vibration signals is extracted.

Here, we demonstrate the implementation and benefits of stochastic resonance in a bistable micromechanical slider. Several research groups have focused on bistable MEMS, using either residual stress (Qiu et al., 2001), buckled beams (Brenner et al., 2003; Sulfridge et al., 2004), initially curved beams (Krylov et al., 2008) or electrostatic forcing (Kuipers et al., 2006). In this work, electrostatic forces are used to obtain bistability in our slider. The implementation of SR in MEMS bistable systems was discussed earlier by Saif (2000), and demonstrated by Ferrari et al. (2010) for energy harvesting applications using a piezoelectric beam converter coupled to permanent magnets. In this chapter, we show that the use of SR can indeed be used to improve the signal-to-noise ratio. We devised a MEMS-slider that is fully voltage-controlled and has completely tunable threshold and noise strength and the possibility to electrostatically apply periodic (subthreshold) forces. Using this device we investigate the impact of noise bandwidth and waveform on the SR behaviour.

The organisation of this chapter is as follows. In the next section, the theory of SR is briefly reviewed and its specific implementation in our MEMS-slider is described. The fabrication process of the MEMS-slider, based on silicon-oninsulator (SOI) technology, is discussed in section 6.3. The experimental section contains the measured mechanical response and the demonstration of SR. Additionally, the impact of noise bandwidth, signal waveform and the properties of SR, which are theoretically complicated to describe, are investigated and the results presented. In section 6.5 , several aspects of stochastic resonance and its 


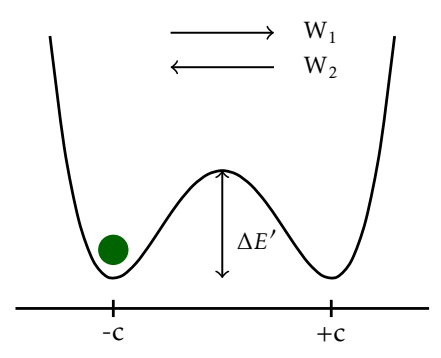

(a) Forcing off.

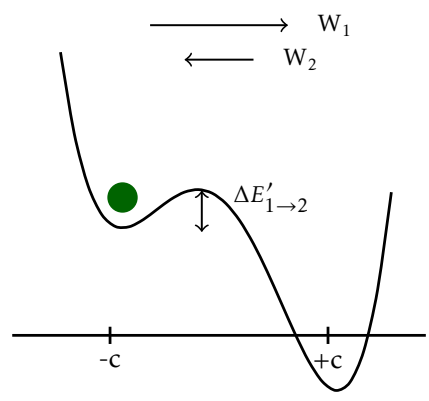

(b) Forcing on.

Figure 6.1: Illustration of external forcing affecting the system behaviour (adapted from (Wellens et al., 2004)). Presence of an external force, right, leads to a skewed potential energy function and causes dissimilarity in the transition probabilities from state $-c$ to $+c$ and vice verse.

application in micromechanical slider-based devices are discussed.

\subsection{Theory and modelling}

\subsubsection{Stochastic resonance}

We briefly summarise the theory of SR following the analysis by Wellens et al. (2004). To implement the mechanism of stochastic resonance, the device has to contain at least two equilibrium points for its position to realize a detection threshold (figure 6.1). In absence of noise and without the presence of an external force, the system will remain in one of the equilibrium states $\pm c$. When applying an external force (i.e. displacing the slider by actuation in our device), the system's energy function becomes asymmetric (tilted), changing the transition probability to move towards the other equilibrium position. In absence of noise, the observable (i.e. the slider-position in our system) only changes when the actuation strength is above a specific threshold level (related to $\Delta E^{\prime}$ ).

To illustrate this effect, consider the double well potential system in figure 6.1, and consider that it is periodically skewed:

$$
E^{\prime}(x, t)=E_{0}^{\prime}(x)+\frac{x}{c} E_{1}^{\prime} \sin \left(\omega_{\mathrm{s}} t\right),
$$

leading to time-dependent energy barriers which become dissimilar for transitions from $-c \rightarrow+c$ and $-c \leftarrow+c$. In the presence of noise, the transition rates $W_{1,2}$, which are the rates from getting from state $-c$ into state $+c$ or vice versa, are given 
by Kramers' formula (Wellens et al., 2004), where $D$ is the rms-noise strength:

$$
W_{1,2} \propto \exp \left[\frac{-\left(\Delta E^{\prime} \pm E_{1}^{\prime} \sin \left(\omega_{\mathrm{s}} t\right)\right)}{D}\right] .
$$

As a result, during certain phases of driving the probability to go from state $-c$ to state $+c$ is enhanced, whereas the opposite transition probability is reduced. Furthermore, in absence of noise the system will not exhibit any transition at all. For large values of $D$ many transitions will occur, suppressing the effect of the small harmonic signal, and the movement being dominated by noise. In between these values there exists an optimum for which the combination of the harmonic signal and noise results in a better detectability of the former. At this optimum, the signal will show quasi periodic behaviour, hence the term stochastic resonance. The advantage of stochastic resonance can be expressed in terms of enhancing the signal-to-noise ratio of the system. For the double well potential system, the signal-to-noise ratio (SNR), which is the signal power divided by the noise power using the system's power spectral density, can be expressed as a function of the noise strength $D$ by the Kramers' rate (Kramers, 1940):

$$
\mathrm{SNR} \propto \frac{1}{D^{2}} \exp \left(\frac{-\Delta E^{\prime}}{D}\right) .
$$

By exploiting the SR-mechanism - thus adding noise — less energy is required to move the system over the energy barrier in one direction whereas the reverse direction shows an increased energy barrier. When the additive noise plus actuation is sufficient to pass the barrier in the direction of the lower threshold, but does not substantially increase the likelihood of passing the barrier from the high threshold direction, the slider movement will quasi lock in with the applied actuation signal. For too low noise power there is no benefit since the combined action of actuation-voltage and noise is not sufficient to pass the threshold, even in the low threshold direction. In case of high noise power, the noise will be dominant and the movement becomes independent of the applied actuation force (Wellens et al., 2004).

With the concept of stochastic resonance described for a two-state system, our micromechanical slider requires to exhibit bistability. In the next subsection, we describe the electromechanical behaviour of the slider using an energy-based approach. We explain how capacitive structures with a positional periodicity allow for achieving this bistability and how the energy barrier and sub-threshold force can both be arbitrarily defined using voltages. 


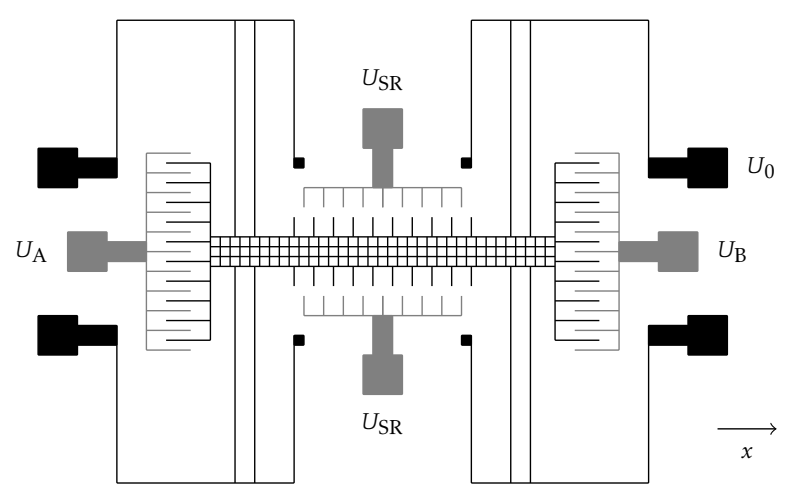

Figure 6.2: Mechanical diagram of the slider.

\subsubsection{Energy function}

A mechanical diagram of the slider is shown in figure 6.2. The slider is suspended by so-called folded flexures, allowing for large displacements of the slider with respect to the width of the beam that determines the system's mechanical stiffness (Legtenberg et al., 1996). The MEMS-slider is electrostatically actuated using the comb drive structures on the left and right sides, with applied voltages $U_{\mathrm{A}}$ and $U_{\mathrm{B}}$ respectively. The desired system's potential, i.e. a double well, or in this case multi well energy function, is achieved through a periodic geometry of the capacitive structures in the middle of the MEMS-slider with the application of a dc-bias voltage $U_{\mathrm{SR}}$, as has been discussed earlier by Adams et al. (1999). These comb-drives have no overlap and only shift sideways to another. This causes their mutual capacitance to become periodic in the displacement coordinate $x$, i.e. $C=C(x)$. Using transduction principles for an electromechanical system affected by a position-dependent and voltage-controlled capacitor, Legendre's transform gives the co-energy $E^{\prime}$ of the system:

$$
E^{\prime}(x, u)=\frac{1}{2} k x^{2}-\frac{1}{2} u^{2} C(x)
$$

where $k$ is the slider's spring constant and $u$ is the applied voltage $u$. Due to the periodicity of $C(x)$ the co-energy $E^{\prime}$ has multiple local extrema. The amplitude of the co-energy profile $E^{\prime}(x)$ is controlled by a judicious choice of the voltage $U_{\mathrm{SR}}$.

Following the electromechanical diagram of figure 6.2, the capacitance varies with the slider's position (Adams et al., 1998). FEM-simulations in COMSOL ${ }^{\circledR}$ show that this capacitance exhibits a positional periodicity, which can be well approximated by a cosine relationship (figure 6.3). Based upon these simulation results, the periodic capacitance in dependence of the slider position $x$ can be 


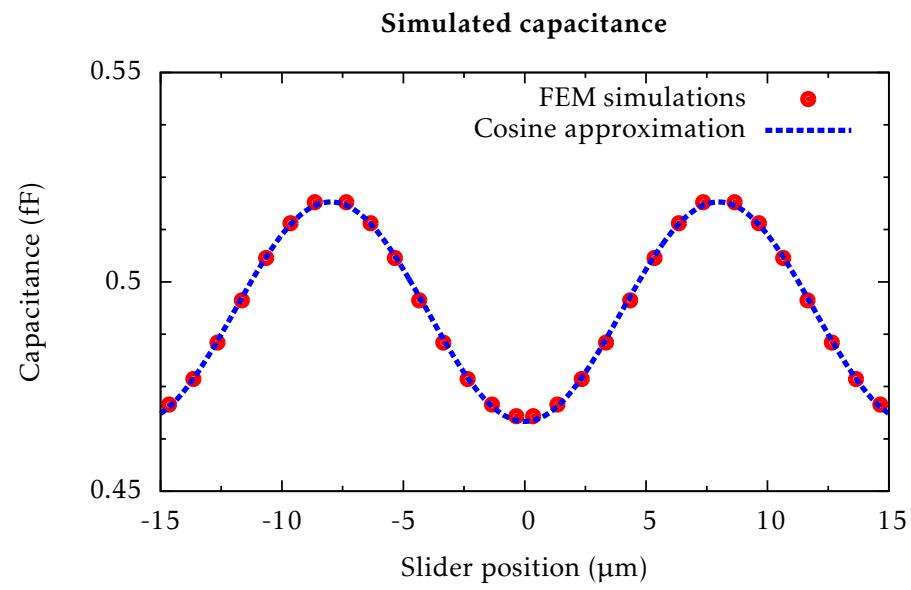

Figure 6.3: Simulation of the position-dependent capacitance, together with an analytical approximation.

approximated by:

$$
C_{\mathrm{SR}}(x) \approx N_{\mathrm{f}}\left[C_{p}-C_{0} \cos \left(\frac{2 \pi x}{\Lambda}\right)\right],
$$

where $\Lambda$ is the comb finger periodicity, $N_{\mathrm{f}}$ is the number of fingers, and $C_{\mathrm{p}}$ and $C_{0}$ are coefficients. Further, the comb-based capacitances left and right for electrostatic actuation also contribute to the system's co-energy function. These capacitances are given by:

$$
C_{\text {left,right }}(x)=N_{\mathrm{a}} \frac{2 \epsilon_{0} h\left(w_{0} \mp x\right)}{g},
$$

where $h$ is the height of a comb finger, $w_{0}$ is the initial overlap in the slider's movement direction between the comb fingers, $x$ is the slider's displacement, $N_{\mathrm{a}}$ is the number of comb fingers and $g$ is the gap between the comb fingers. The total co-energy function can be expressed as:

$$
E^{\prime}(x)=\frac{1}{2} k x^{2}-\frac{1}{2} U_{\mathrm{SR}}^{2} C_{\mathrm{SR}}(x)-\frac{1}{2} U_{\mathrm{A}}^{2} C_{\text {left }}(x)-\frac{1}{2} U_{\mathrm{B}}^{2} C_{\text {right }}(x) .
$$

The slider will be operated in a push-pull configuration by choosing:

$$
U_{\mathrm{A}, \mathrm{B}}=U_{\mathrm{dc}} \pm U_{\mathrm{ac}} \cos (\omega t),
$$

where $U_{\mathrm{dc}}$ is the applied dc-bias voltage, $U_{\mathrm{ac}}$ is the amplitude of the ac voltage, 
and $\omega$ is the actuation frequency. The co-energy function can be simplified to:

$$
E^{\prime}(x, t)=\frac{1}{2} k x^{2}+\frac{1}{2} U_{\mathrm{SR}}^{2} N_{\mathrm{f}} C_{0} \cos \left(\frac{2 \pi x}{\Lambda}\right)+U_{\mathrm{dc}} U_{\mathrm{ac}} \cos (\omega t) N_{\mathrm{a}} \frac{4 \epsilon_{0} h}{g} x+E_{\mathrm{off}}^{\prime},
$$

where $E_{\text {off }}^{\prime}$ is a position-independent co-energy term:

$$
E_{\text {off }}^{\prime}=-\frac{1}{2} U_{\mathrm{SR}}^{2} N_{\mathrm{f}} C_{p}-\left(U_{\mathrm{dc}}^{2}+U_{\mathrm{ac}}^{2} \cos (\omega t)^{2}\right) N_{\mathrm{a}} \frac{2 \epsilon_{0} h w_{0}}{g} .
$$

As can be seen from (6.9), the co-energy function $E^{\prime}(x, t)$ consists of four terms. The first term is proportional to $x^{2}$, as a result of the system's mechanical stiffness. Periodicity in the system's co-energy function is given by the second term, which exhibits a cosine-relationship with respect to the position $x$. Thirdly, a so-called tilt in the co-energy function is achieved by having the term proportional to $x$ and simultaneously depending on time $t$. The last term $E_{\text {off }}^{\prime}$ is an offset in the energy function, depending on both the periodic capacitive structures and the comb drives for electrostatic actuation.

Using the slider's parameters from table 6.1, the impact of changing the socalled well voltage $U_{\mathrm{SR}}$ is shown in figure 6.4 in absence of an actuation force ( $U_{\mathrm{dc}}$ $\left.=U_{\mathrm{ac}}=0 \mathrm{~V}\right)$. In case no voltage is applied to the periodic capacitive structures $\left(U_{\mathrm{SR}}=0 \mathrm{~V}\right)$ a parabolic profile having one equilibrium point at $x=0$ is observed, as a result from the intrinsic mechanical stiffness $K$. By increasing $U_{\mathrm{SR}}$ the coenergy profile flattens around $x=0$, and shows for high values of $U_{\mathrm{SR}}$ multiple equilibria (e.g. $U_{\mathrm{SR}}=60 \mathrm{~V}$ ). The latter case corresponds to the profile without external forcing shown in figure 6.1a.

The effect of the application of both $U_{\mathrm{SR}}=60 \mathrm{~V}$ and an external force is demonstrated in figure 6.5. The co-energy of the system is shown in absence of a force, and in presence of both a relative weak and relative strong force. On application of a force the co-energy function gets tilted, resulting in a decreased barrier height for a transition from right to left, similar to figure $6.1 \mathrm{~b}$. Simultaneously, the barrier height for going from left to right increases. In case of a relative weak (sub-threshold) force additional noise is required to move the system from state ' $+c^{\prime}$ (right) to '-c' (left). A strong force will not require the presence of added noise, as the corresponding co-energy function contains only one global minimum. Hence, whether an external force is sub-threshold or not depends on the barrier height $\Delta E^{\prime}$.

The total electromechanical behaviour of the slider is described by the coenergy function given in (6.9). To evaluate this expression, several parameters, like the spring constant $k$ and capacitive parameter $C_{0}$, need to be determined 


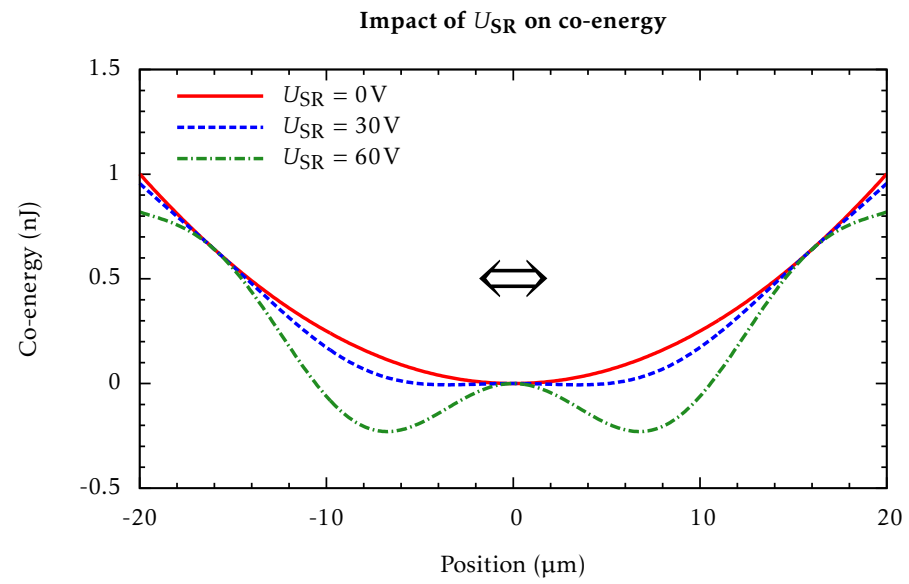

Figure 6.4: Creating bistability by increasing the well voltage $U_{\mathrm{SR}}$.

Tilting by electrostatic actuation

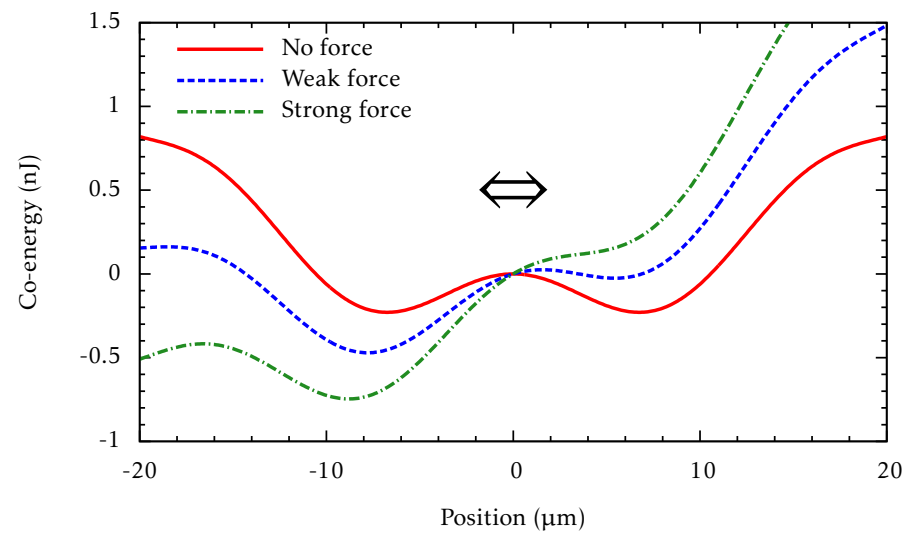

Figure 6.5: Increasing and decreasing the transition rates $W_{1,2}$ by external forcing.

experimentally. To this end, the slider dynamics are described for small slider displacements using a Duffing-based approach in the next subsection. By measuring the frequency response of the slider for such displacements and using the corresponding model, the parameters $k$ and $C_{0}$ can be found. 


\subsubsection{Mechanics}

To experimentally determine slider parameters, the slider is actuated in such a way that the displacements are small with respect to the periodicity $(\Lambda)$ of the capacitive structures. In that case, the response of the slider can be described by the equation of motion for a non-linear spring (the well-known Duffing equation):

$$
m \frac{\mathrm{d}^{2} x}{\mathrm{~d} t^{2}}+\gamma \frac{\mathrm{d} x}{\mathrm{~d} t}+k_{1} x+k_{3} x^{3}=F_{\omega} \cos (\omega t)
$$

with

$$
\gamma=\frac{\sqrt{k_{0} m}}{Q}
$$

where $m$ is the slider's mass, $k_{0}$ is the intrinsic linear spring coefficient, $k_{1}$ is the total linear spring coefficient, $k_{3}$ is the cubic spring coefficient, $Q$ is the quality factor, $\omega_{0}$ is the resonance frequency, and $F_{\omega}$ is the amplitude of the driving force with frequency $\omega$. The Duffing equation can be solved using a perturbation analysis around its resonance frequency $\omega_{0}\left(=\sqrt{k_{0} / m}\right)$. Following the analysis of Kaajakari et al. (2004), an implicit equation results as a solution for the slider's displacement amplitude $X_{0}$ :

$$
X_{0}=\frac{F_{\omega}}{m \sqrt{\left(\omega^{2}-\omega_{0}^{\prime 2}\right)^{2}+\left(\frac{\omega \omega_{0}^{\prime}}{Q}\right)^{2}}},
$$

where

$$
\omega_{0}^{\prime}=\omega_{0}+\eta X_{0}^{2}, \quad \text { and } \quad \eta=\frac{3 k_{3}}{8 k_{1}} \omega_{0} .
$$

By using a Taylor expansion for $C(x)$, the voltage-dependent spring coefficients $k_{1}$ and $k_{3}$ are calculated:

$$
k_{1} \approx k_{0}-2 N_{\mathrm{f}} U_{\mathrm{SR}}^{2} C_{0}\left(\frac{\pi}{\Lambda}\right)^{2}, \quad \text { and } \quad k_{3} \approx \frac{4}{3} N_{\mathrm{f}} U_{\mathrm{SR}}^{2} C_{0}\left(\frac{\pi}{\Lambda}\right)^{4}
$$

To obtain bistability, the system must be unstable around $x=0$ (figure 6.4). Following our analysis, this requires the spring constant $k_{1}$ to be zero or lower, leading to the condition for the dc-bias voltage $U_{\mathrm{SR}}$ :

$$
U_{\mathrm{SR}} \geq \frac{\Lambda}{\pi} \sqrt{\frac{k_{0}}{2 N_{\mathrm{f}} C_{0}}},
$$

which, using the parameters listed in table 6.1, corresponds to a dc-bias voltage 
$U_{\mathrm{SR}}$ of $46.7 \mathrm{~V}$. Under this condition, the micromechanical slider will display bistability and consequently SR can be exploited.

In the next subsection, we investigate to what extent adding noise can help to increase the signal-to-noise ratio. To this end, expressions for the signal-tonoise ratio are derived for SR-systems. Although our micromechanical slider is expected to be clearly underdamped by having a high quality factor, we describe the signal-to-noise ratio for both the classical (overdamped) regime as well as for the undamped regime, so one can compare the results for the corresponding signal-to-noise ratios.

\subsubsection{Signal-to-noise ratio}

To calculate the signal-to-noise ratio and gain some insight in the slider's SR performance, the slider's mechanics are again approximated by a Duffing-based equation. Using this approximation the system will have only three energy extrema rather than the multiple energy extrema that our slider structure can have. However, for displacements that are within $75 \%$ of the finger periodicity $\Lambda$ this approach leads to an error for the energy function that falls within reasonable limits $(<12 \%)$. Notice that by following this approach the calculated location of the equilibria $\pm c$ are underestimated relative to the exact solution.

In most SR-bistable systems, the corresponding signal-to-noise ratio is calculated by assuming that the damping parameter $\gamma$ is strong and the inertial effects can be neglected. In this case, the mechanics of the slider are described by the periodically modulated Langevin equation (Gammaitoni et al., 1998):

$$
\frac{\mathrm{d} x}{\mathrm{~d} t}=a x-b x^{3}+A \cos (\omega t)+\xi(t),
$$

where $\xi(t)$ is Gaussian white noise with zero mean, unit variance and intensity $D$. Further, the parameters $a, b$ and $A$ are defined as:

$$
a=-\frac{k_{1}}{\gamma}, \quad b=\frac{k_{3}}{\gamma}, \quad A=\frac{F_{\omega}}{\gamma} .
$$

By following the analysis of (McNamara and Wiesenfeld, 1989; Gammaitoni et al., 1998; Li et al., 2013), the transition between states is given by the Kramers' rate $r_{\mathrm{k}}$ :

$$
r_{\mathrm{k}}=\frac{a}{\sqrt{2 \pi}} \exp \left(-\frac{\Delta E^{\prime}}{D}\right),
$$

with the barrier height $\Delta E^{\prime}$ defined as: 


$$
\Delta E^{\prime}=\frac{a^{2}}{4 b}
$$

The signal-to-noise ratio $\mathrm{SNR}_{\gamma}$ for an overdamped bistable system — under the condition of a sub-threshold force $A-$ is then given by:

$$
\mathrm{SNR}_{\gamma}=\frac{a \pi A^{2}}{2 b D^{2}} r_{k}, \quad \text { with } \quad A<\frac{1}{4} \sqrt{\frac{a^{3}}{b}} .
$$

This expression has a similar proportionality to the noise intensity as the signalto-noise ratio based on the Kramers' rate defined earlier in (6.3). Although there is no direct frequency-dependency in SNR, its expression is only valid under the condition of the adiabatic limit (Gammaitoni et al., 1998)):

$$
r_{\mathrm{k}} \leq 2 f_{0}, \quad \text { where } \quad f_{0}=\frac{1}{2 \pi} \sqrt{\frac{k_{0}}{m}} .
$$

The result of (6.21) is only valid in case the system is significantly overdamped. However, the mechanism of SR can also exist for systems operating in the underdamped regime (Gammaitoni et al., 1989). Its implementation in underdamped bistable systems was explored earlier by several research groups (Alfonsi et al., 2000; Zhang, 2001; Ray and Sengupta, 2006; Kenfack and Singh, 2010; Xu et al., 2013), in which the governing equation is the periodically modulated undamped Langevin equation:

$$
\frac{\mathrm{d}^{2} x}{\mathrm{~d} t^{2}}=a x-b x^{3}+A \cos (\omega t)+\sqrt{2 D} \xi(t),
$$

where $D$ is the noise intensity, and $\xi(t)$ is a Gaussian white noise with zero mean and unit variance. The parameters $a, b$ and $A$ change with respect to (6.17) and for the undamped case get redefined as:

$$
a=-\frac{k_{1}}{m}, \quad b=\frac{k_{3}}{m}, \quad A=\frac{F_{\omega}}{m} .
$$

The signal-to-noise ratio $\mathrm{SNR}_{\mathrm{m}}$ for the undamped bistable system was calculated by Li et al. (2013) and is given by:

$$
\mathrm{SNR}_{\mathrm{m}}=\frac{a A^{2}}{4 b D^{2}} \sqrt{\frac{a}{8}} \exp \left(-\frac{a^{2}}{4 b D}\right) \times\left[1-\frac{2 a^{2} A^{2} \exp \left(-\frac{a^{2}}{2 b D}\right)}{4 a b D^{2} \exp \left(-\frac{a^{2}}{2 b D}\right)+2 b D^{2} \pi^{2} \omega^{2}}\right]^{-1}
$$

This expression requires the force $F_{\omega}$ to be sub-threshold and therefore $F_{\omega}$ needs 


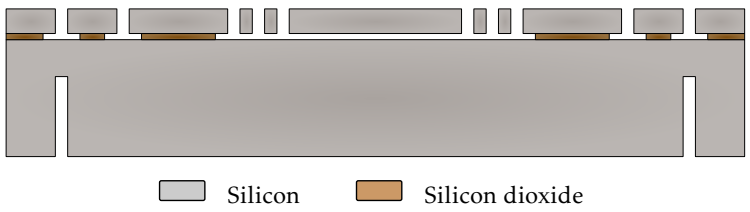

Figure 6.6: Cross-sectional view of the sensor.

to satisfy the condition:

$$
F_{\omega}<\frac{1}{4} \sqrt{\frac{-k_{1}^{3}}{k_{3}}} .
$$

Although the expression for $\mathrm{SNR}_{\mathrm{m}}$ is much more complicated than the expression for the signal-to-noise ratio in overdamped bistable systems, both expressions state that there exists an optimal noise intensity for achieving the best signal-tonoise ratio. For both (6.21) and (6.25), the SNR has to be multiplied by $\mathrm{Hz}^{-1}$ due to the usage of the Dirac delta function for calculating the signal power in the power spectrum (see e.g. (McNamara and Wiesenfeld, 1989)).

\subsection{Fabrication}

For implementation of the SR-scheme in a MEMS-slider, both the design and fabrication are inspired by the work of Kuijpers et al. (2006). The slider is fabricated from a $<100>$ single-crystal highly boron-doped silicon-on-insulator (SOI) wafer, with a $25 \mu \mathrm{m}$ thick device layer and an oxide thickness of $2 \mu \mathrm{m}$. The slider structures are created by deep reactive-ion etching (DRIE) using a Boschprocess (Laermer and Schilp, 1994), optimized for reduction of RIE-lag (Jansen et al., 1997) over the wafer. The structures are released using vapour HF to etch the buried oxide layer (Holmes and Snell, 1966). A cross-sectional view of the SOI wafer after fabrication is shown in figure 6.6.

The fabrication result is shown in figure 6.7 , where the capacitive periodic structures, folded flexures and comb drive structures are visible. The comb finger thickness, distance between the top of moving and non-moving finger, and beam width in the given design are all $4 \mu \mathrm{m}$. Further, the folded flexure beam length is about $600 \mu \mathrm{m}$. 


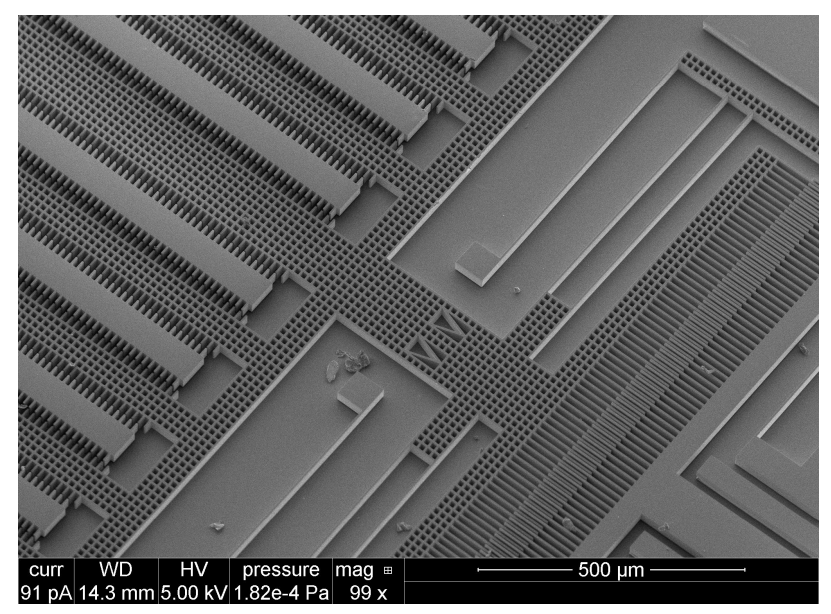

Figure 6.7: SEM picture of the electrostatically driven slider.

\subsection{Experimental}

In this section, first the electromechanical parameters are experimentally determined by measuring the slider's frequency response. With these parameters known, one can evaluate the expressions derived in section 6.2. Then, the method for measuring and quantifying SR in our micromechanical slider is explained. Subsequently, measurements are performed and described for investigation of the role of noise bandwidth on SR and the applicability of SR to non-sinusoidal waveforms.

\subsubsection{Frequency response}

Actuation of the slider is performed electrostatically using comb drive structures in a push-pull configuration (see (6.8) and figure 6.2). Furthermore, the height of the energy barrier is controlled by a dc-bias voltage $U_{\mathrm{SR}}$ applied to the periodic capacitive structures. First, the sliders's frequency response is obtained by measuring the displacement optically using the in-plane measurement mode of the Polytec MSA-400 system (Polytec, 2005). The slider is electrostatically driven using a waveform generator (Agilent 33220A) for generation of the harmonic voltage with frequency $\omega$. A dc-power supply (Delta Power Supply E018-0.6 D) was used to provide an offset to the harmonic voltage. A high-voltage amplifier (EsyLAB LM3325) was used for amplification of the driving voltage to achieve voltages of up to $100 \mathrm{~V}$. The dc-bias voltage $U_{\mathrm{SR}}$ was supplied by a high voltage power supply (Delta Power Supply E300-0.1). 


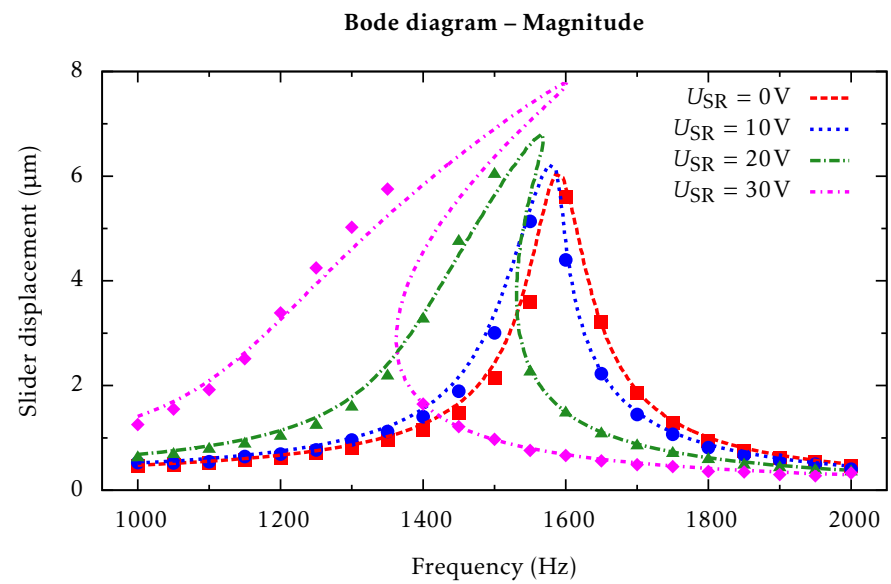

Figure 6.8: Frequency response of the slider for different well voltages (measurements shown by symbols, solid lines represent the analytical model, equations (6.13)-(6.15)).

Table 6.1: Experimental values of the slider and capacitive structures.

\begin{tabular}{lll}
\hline Quantity & Symbol & Value \\
\hline Mechanical parameters & & \\
\hline Spring constant & $k_{0}$ & $8.52 \mathrm{Nm}^{-1}$ \\
Mass & $m$ & $8.54 \times 10^{-8} \mathrm{~kg}$ \\
\hline Slider specifications & & \\
\hline Quality factor & $\mathrm{Q}$ & 21 \\
Resonance frequency & $f_{0}$ & $1.59 \mathrm{kHz}$ \\
\hline Capacitive structures & & \\
\hline Number of fingers & $N_{\mathrm{f}}$ & 1920 \\
Finger periodicity & $\Lambda$ & $16 \mu \mathrm{m}$ \\
Initial capacity & $C_{\mathrm{p}}$ & $4.93 \times 10^{-16} \mathrm{~F}$ \\
Capacity coefficient & $C_{0}$ & $2.64 \times 10^{-17} \mathrm{~F}$ \\
\hline
\end{tabular}

The resulting response for four values of the dc-bias voltage is shown in Figure 6.8, which is in good agreement with the analytical model based upon (6.13) and (6.15), using the values listed in table 6.1 and a force $F_{\omega}$ of $2.41 \times 10^{-6} \mathrm{~N}$. We observe that on increasing the dc-bias voltage, the system exhibits the expected spring softening with a cubic spring hardening contribution. The resonance frequency $f_{0}$ of the system was found to be $1.59 \mathrm{kHz}$ and the quality factor 21 .

A summary of the experimentally derived values, also used for model calculations, is given in table 6.1. 


\subsubsection{SR-experiments}

To measure the slider displacements during experiments for stochastic resonance its position was measured by means of a dedicated camera (Prosilica CV1280) in combination with real-time LabVIEW ${ }^{\circledR}$ image capture. Using this configuration, the position of the slider could be measured with a time interval of $5 \mathrm{~ms}$. Subsequently, the slider's displacement $x$ was calculated off-line using the Image and Video Processing toolbox of MATLAB ${ }^{\circledR}$. In this configuration, the displacement could be estimated with an accuracy of approximately $\pm 250 \mathrm{~nm}$. Although it is possible to obtain a higher displacement resolution by e.g. using a discrete Fourier analysis (Yamahata et al., 2010), the mentioned resolution within a displacement range of about the finger periodicity $(\Lambda=16 \mu \mathrm{m})$ is sufficient to determine the slider's state, i.e. $\pm c$. As a consequence of the Nyquist criterion, the maximum measurable frequency of the slider's displacement had to be chosen lower than $100 \mathrm{~Hz}$.

SR-experiments with the micromechanical slider were conducted using a displacement threshold of $2 \mu \mathrm{m}$ as a representative discrimination between states ' -1 ', ' 0 ' and ' 1 '. However, the choice of threshold is somewhat arbitrary since the slider is allowed to move up to a displacement of $8 \mu \mathrm{m}$ for the used actuation voltages. To investigate the impact of the choice of this threshold on the measured signal-to-noise ratio, one of the SR-measurements (shown in figure 6.10) was evaluated for various values of the displacement threshold. The results of this evaluation are shown in figure 6.9. It can be observed that for threshold values up to about $5 \mu \mathrm{m}$ the determined SNR is hardly affected by the choice of threshold, but for values close to half of the systems' periodicity, i.e. $8 \mu \mathrm{m}$, the SNR clearly deteriorates. In the results presented below the thresholds were either $\pm 2 \mu \mathrm{m}$ or $\pm 5 \mu \mathrm{m}$.

For SR-experiments, the system was brought into a sub-threshold mode $\left(k_{1}<0\right)$ by judiciously chosen applied voltages $\left(U_{\mathrm{dc}}=50 \mathrm{~V}, U_{\mathrm{ac}}=10 \mathrm{~V}\right)$. For all experiments, an actuation frequency of $20 \mathrm{~Hz}$ was chosen, which is well below the resonance frequency $f_{0}$ of the slider and satisfying the Nyquist criterion. Noise is added by application of a white noisy voltage $U_{\text {noise }}$ to both actuation comb drives, giving for the net actuation voltages on left and right sides:

$$
U_{\mathrm{A}, \mathrm{B}}=U_{\mathrm{dc}} \pm U_{\mathrm{ac}} \cos (\omega t) \pm U_{\text {noise }}
$$

In the presented slider the finger periodicity $\Lambda$ is $16 \mu \mathrm{m}$. The capacitance is the largest when the mutual distance between the moving and non-moving fingers is smallest (figure 6.3), coinciding with the positions of the equilibria, allowing the slider to move $\pm 8 \mu \mathrm{m}$ for the used voltages. In the experiments the measured 


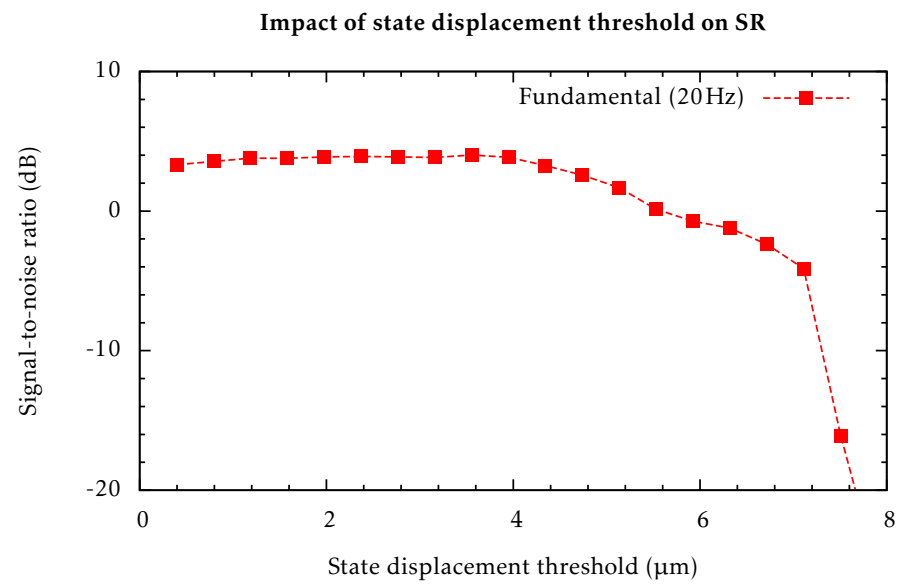

Figure 6.9: Influence of the choice of state displacement threshold on the signal-to-noise ratio when exploiting $S R\left(U_{S R}=60 \mathrm{~V}\right)$. The slider is driven sinusoidally at a frequency of $20 \mathrm{~Hz}$.

position of the slider is interpreted as a state of the system. For positions around the slider's zero position $(x=0)$, the slider output corresponds to a ' 0 '. Following the two-state system model, being in the left equilibrium $(x<$ about $-2 \mu \mathrm{m})$ corresponds to $-c$ giving output ' -1 ' and similarly the right equilibrium $(x>$ about $2 \mu \mathrm{m}$ ) corresponds to $+c$ giving output ' 1 '. Subsequently a Fast Fourier Transform (FFT) is performed on the logical output and the resulting SNR is calculated by:

$$
\mathrm{SNR}=\frac{Y_{k}^{2}}{\sum_{n=0}^{N} Y_{n}^{2}-\sum_{i=0}^{I} Y_{(2 i+1) \times k}^{2}-Y_{0}^{2}},
$$

where $Y_{k}$ is the signal amplitude at $20 \mathrm{~Hz}$. The noise power is calculated by computing the total signal power, excluding the signal frequency, higher order frequencies and the dc-component $Y_{0}^{2}$.

\subsubsection{Noise bandwidth}

First, the impact of the noise bandwidth on the resulting SNR as function of the noise strength $D$ while applying a sinusoidal actuation voltage is investigated. The noise bandwidth $\Delta f$ was changed by adding an adjustable band pass filter (SR $650)$ to the experimental setup. The results of these SR-experiments are shown in 


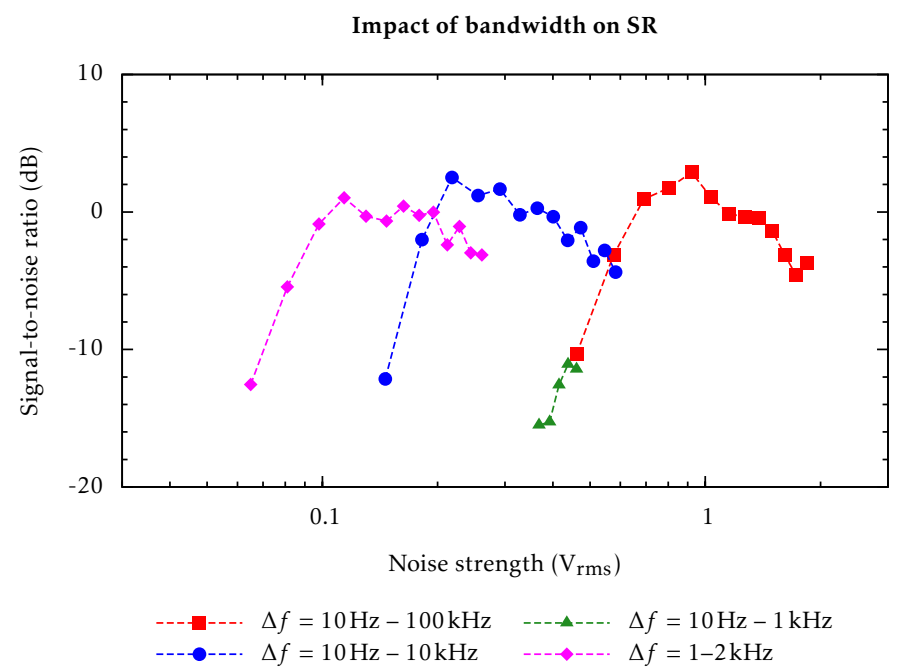

Figure 6.10: Increasing the signal-to-noise ratio by adding white noise to one of the actuator combs $\left(U_{\mathrm{SR}}=60 \mathrm{~V}\right)$. Notice that for the case $\Delta f=10 \mathrm{~Hz}-1 \mathrm{kHz}$ (green line) the electronic equipment could not deliver larger noise intensities.

figure 6.10. In absence of noise, the slider's position is fixed to one of the equilibria $(+c$ or $-c)$, thus confirming that the actuation force is below the system's threshold. By adding noise to the actuation comb drives, i.e. moving the slider over the energy barrier, the SNR clearly increases. For large noise values, the slider's displacement becomes too noisy and, hence, the SNR is seen to decrease.

We observe that by reducing the noise bandwidth $\Delta f$ from $10 \mathrm{~Hz}-100 \mathrm{kHz}$ (shown in red) to a bandwidth of $10 \mathrm{~Hz}-10 \mathrm{kHz}$ (shown in blue), the shape of the resulting SNR-curve is similar, but less noise is required to achieve the best SNR. By further reducing the bandwidth to sub-resonance frequencies of $10 \mathrm{~Hz}-1 \mathrm{kHz}$ (shown in green), only for large rms-values of noise movement of the slider was observed. However, this movement turns out to be rather noisy and results in poor SNR-values. By choosing the noise bandwidth to be $1-2 \mathrm{kHz}$, being around the slider's resonance frequency of $1.59 \mathrm{kHz}$, even lower noise values can be used to achieve good SNR-values (shown in magenta) compared to measurements within a noise bandwidth of $10 \mathrm{~Hz}-10 \mathrm{kHz}$.

\subsubsection{Non-sinusoidal waveforms}

We showed that the mechanism of SR can be exploited to improve the SNR for a sinusoidal waveform. However, we also investigated the impact of SR on having 


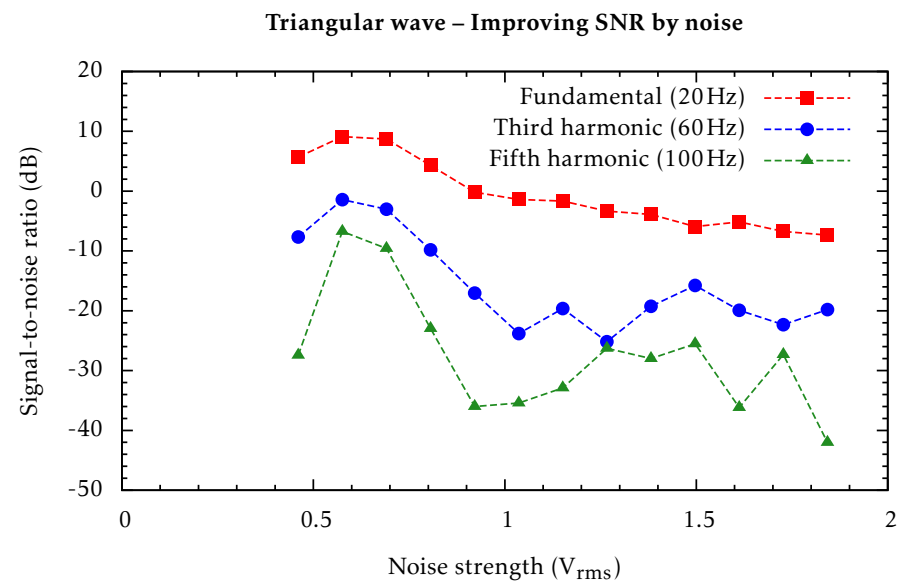

Figure 6.11: Exploiting SR for a triangular wave based voltage $\left(U_{S R}=60 \mathrm{~V}\right)$.

non-sinusoidal waveforms. First, SR-experiments were performed for the case of a triangular waveform, given by:

$$
u_{\mathrm{t}}(t)=\frac{8}{\pi^{2}} U_{0}\left(\sin (\omega t)-\frac{1}{9} \sin (3 \omega t)+\frac{1}{25} \sin (5 \omega t)+\ldots\right),
$$

where $U_{0}$ is equal to the used amplitude $U_{\mathrm{ac}}$ of $10 \mathrm{~V}$ and $\omega$ is the fundamental frequency of $20 \mathrm{~Hz}$. The obtained SNR-values for the case of a noise bandwidth $\Delta f$ from $10 \mathrm{~Hz}-100 \mathrm{kHz}$ are shown in figure 6.11, where the fundamental, third and fifth harmonic of the signal are shown.

Similar to figure 6.10, we observed that for low noise rms-values no state transitions occurred. For higher noise rms-values, the SNR of both the fundamental and higher harmonic frequency components exhibits a decrease with increasing noise strength. Furthermore, the fundamental frequency of $20 \mathrm{~Hz}$ shows a significant higher SNR compared to the third harmonic at a frequency of $60 \mathrm{~Hz}$.

A comparable experiment was performed by application of a square waveform actuation voltage, given by:

$$
u_{\mathrm{s}}(t)=\frac{4}{\pi} U_{0}\left(\sin (\omega t)+\frac{1}{3} \sin (3 \omega t)+\frac{1}{5} \sin (5 \omega t)+\ldots\right) .
$$

Also here a noise bandwidth $\Delta f$ from $10 \mathrm{~Hz}-100 \mathrm{kHz}$ was used, an amplitude $U_{0}$ of $10 \mathrm{~V}$ and a fundamental frequency of $20 \mathrm{~Hz}$. The results are shown in figure 6.12, where the fundamental, third and fifth harmonic of the signal are shown.

We observe that the SNR of the fundamental frequency is significantly larger than the higher order harmonics. However, in contrast to the sinusoidal and 


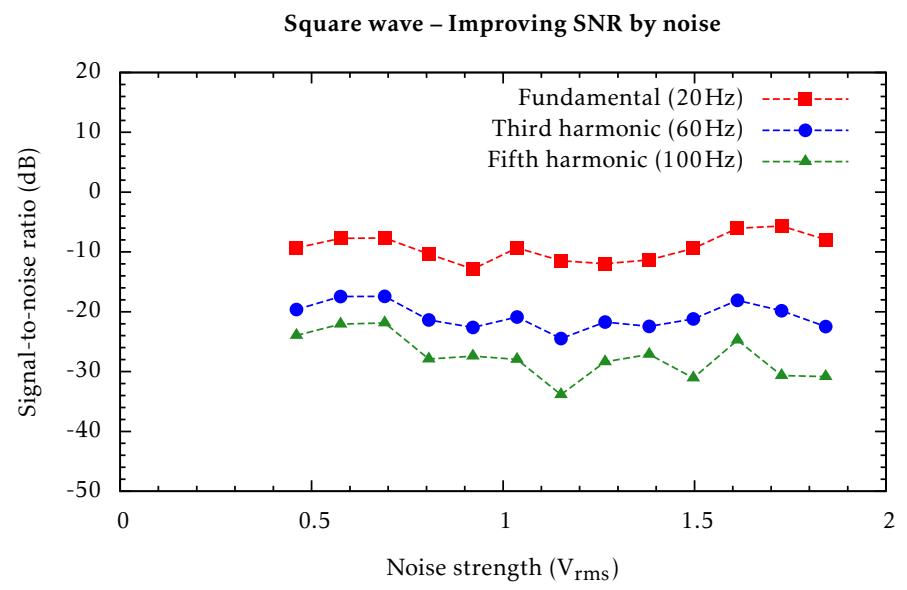

Figure 6.12: Exploiting SR for a square wave based voltage $\left(U_{S R}=65 \mathrm{~V}\right)$.

triangular wave experiments, the obtained SNR-values are relatively low. Also, there is no clear optimal noise strength $D$ for achieving the best signal-to-noise ratio.

\subsection{Discussion}

\subsubsection{Advantage of SR}

To explain why SR can be used to improve the signal-to-noise ratio and the ability to measure sub-threshold forces we turn to figure 6.13. In this graph, a subthreshold sinusoidal slider displacement is shown (in blue) with a displacement amplitude of $2 \mu \mathrm{m}$. By setting the threshold to e.g. $5 \mu \mathrm{m}$ to define the states ' $\pm \mathrm{c}$ ' and setting the well voltage $U_{\mathrm{SR}}$ to $0 \mathrm{~V}$, the output remains zero. As a consequence, the measured signal is zero, giving a (theoretically) infinitely low signal-to-noise-ratio.

Now, the slider is brought into the previously described SR-configuration by increasing the voltage $U_{\mathrm{SR}}$ to $60 \mathrm{~V}$ and adding white noise with a bandwidth of $10 \mathrm{~Hz}-100 \mathrm{kHz}$ to the slider's comb drives, the resulting slider movement (in red) clearly exhibits transitions between states $-c$ and $+c$. In contrast to the measurements with $U_{\mathrm{SR}}$ is $0 \mathrm{~V}$, a 'quasi-digital' signal with a fundamental frequency of $20 \mathrm{~Hz}$ results, giving a finite signal-to-noise ratio. Therefore, by exploiting SR it is possible to measure sub-threshold forces in the discussed micromechanical slider. 


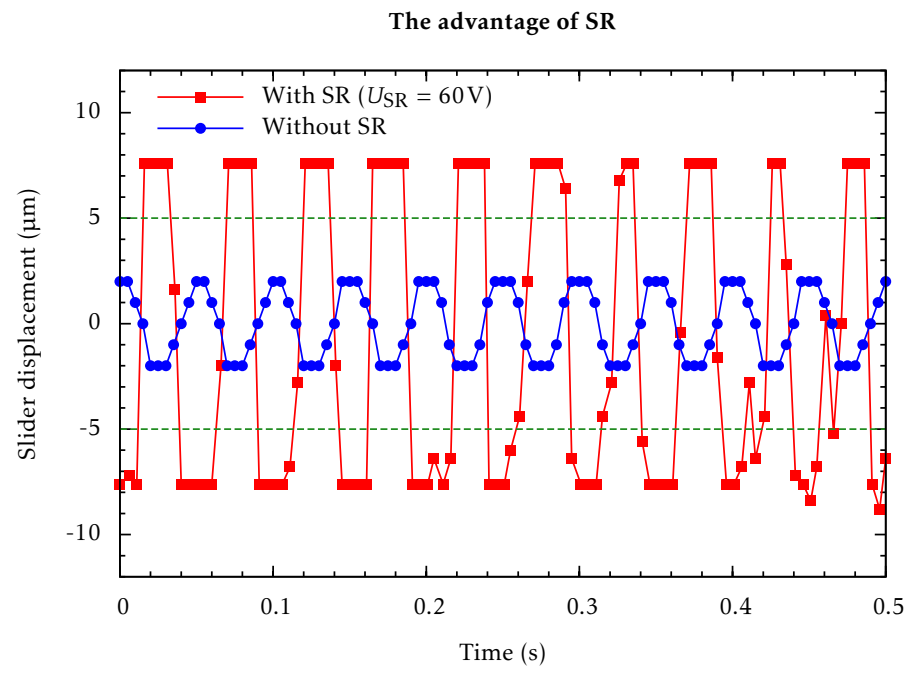

Figure 6.13: Increasing the signal-to-noise ratio by adding white noise to the actuator combs $\left(U_{\mathrm{SR}}=60 \mathrm{~V}\right)$.

\subsubsection{Waveforms}

Following figures 6.10, 6.11 and 6.12, the principle of SR allows to increase the signal-to-noise ratio of common periodic waveforms: sine, triangular and square. Specially for a sinusoidal and triangular wave-based signal, the best signal-to-noise ratio is achieved for a judicious amount of added white noise given the chosen threshold, as predicted by Kramers' formula shown by (6.3). However, for a square wave based voltage transitions between the states $\pm c$ occur for adding noise, but there is no clear optimal amount of added white noise for achieving the best signal-to-noise ratio. This can be explained by the fact that the higher harmonics of a square wave roll off much slower than in a triangular wave (proportional to just the inverse of the harmonic number as opposed to the square inverse). Considering the frequency content of a triangular wave, its third harmonic has an amplitude of just $1 / 9$ of the fundamental frequency component, whereas in a square wave this component is still $1 / 3$. As a result, a triangular wave more closely resembles a sine wave than to a square wave, and therefore its SR properties are better described by current theory.

We observe in figure 6.11 that the SNR for the fundamental frequency is generally about $10 \mathrm{~dB}$ higher than for the third harmonic at $60 \mathrm{~Hz}$. Since in the spectrum $20 \mathrm{~dB}$ coincides with a factor 10 , there is about a factor 3 between the fundamental frequency component and its third harmonic. From (6.30), it roughly 
represents a square wave. Considering the example of SR (figure 6.13), this does not come as a surprise; in this figure, the red curve is based upon a sinusoidal external force, but transforms by application of SR into a 'quasi-digital' signal having characteristics of a square wave.

In general, by considering figures 6.10, 6.11 and 6.12, the applied common periodic waveforms (sine, triangular and square) will all three give a square-wave like response when exploiting stochastic resonance. As a consequence, it is possible to measure the (fundamental) frequency of these periodic waveforms, but their waveform properties will be hard to distinguish, or not distinguishable at all, due to a strong distortion of higher harmonics.

\subsubsection{Noise bandwidth}

A strong impact of noise bandwidth $\Delta f$ on the required added noise was observed in figure 6.10. By reduction of the noise bandwidth from about $100 \mathrm{kHz}$ to about $10 \mathrm{kHz}$, the required effective noise strength was reduced by approximately 3 times. This value coincides roughly with the shift of the SNR-curve in figure 6.10 (from red to blue), indicating that the noise in a frequency band of $10-100 \mathrm{kHz}$ has little impact on the slider's behaviour. This fact can be explained by considering the slider's mechanical frequency response, since the resonance frequency was measured to be below $2 \mathrm{kHz}$. Having a mechanical roll-off of $20 \mathrm{~dB} /$ decade, these rather high frequencies have negligible impact. He et al. (2007) showed that by exploiting SR, a non-linear low-pass filter characteristic for the input is introduced, removing high frequency contributions from the added white noise. Thus, adding noise with frequencies larger than the system's resonance frequency does not help to increase the signal-to-noise ratio by SR.

Further reduction of the noise bandwidth, when excluding the frequency region around resonance, almost no signal is observed. Apparently, the region around resonance is of great importance when applying noise to a second order mechanical system, especially in the case of our slider since it has a high quality factor of 21. This hypothesis is confirmed by choosing the noise bandwidth around resonance $(\Delta f=1-2 \mathrm{kHz})$ and observing that an even lower noise strength is required (reduction by about a factor 2) to achieve the best signal-to-noise ratio. This needs not come as a surprise; the electrically added noise is of no consequence as long as it does not result in mechanical noise and it is the sliders transfer function that determines how much of the first is transformed in the latter. 


\subsection{Conclusions}

By exploiting the mechanism of stochastic resonance (SR) in a voltage-controlled micromechanical slider, the signal-to-noise ratio can be increased by adding white noise. We implemented stochastic resonance by controlling the strength of position-dependent capacitive wells by a dc-bias voltage, operating the device in push-pull mode by electrostatic actuation and adding a judicious amount of white noise to the actuation comb drives. We showed that the use of SR allows for detection of sub-threshold forces. Further, it is demonstrated that the noise bandwidth has a clear impact on the required optimal noise strength. The mechanism of SR is implemented for several types of periodic waveforms. The fundamental frequency could be resolved in all cases, but the type of waveform (sine, triangular or square) turned out to be irresolvable using SR.

\section{References}

Adams, S. G., Bertsch, F. M., Shaw, K. A., and MacDonald, N. C. (1998). Independent tuning of linear and nonlinear stiffness coefficients. J. Microelectromech. S., $7(2): 172-180$.

Adams, S. G., Wang, Y. C., Macdonald, N. C., and Thorp, J. S. (1999). Multistable tunable micromechanical resonators. Patent. US 5914553.

Alfonsi, L., Gammaitoni, L., Santucci, S., and Bulsara, A. R. (2000). Intrawell stochastic resonance versus interwell stochastic resonance in underdamped bistable systems. Phys. Rev. E, 62(1):299-302.

Almog, R., Zaitsev, S., Shtempluck, O., and Buksa, E. (2007). Signal amplification in a nanomechanical Duffing resonator via stochastic resonance. Appl. Phys. Lett., 90:013508.

Badzey, R. L. and Mohanty, P. (2005). Coherent signal amplification in bistable nanomechanical oscillators by stochastic resonance. Nature, 437:995-998.

Bahar, S. and Moss, F. (2004). Stochastic resonance and synchronization in the crayfish caudal photoreceptor. Math. Biosci., 188:81-97.

Benzi, R., Parisi, G., Sutera, A., and Vulpiani, A. (1982). Stochastic resonance in climate change. Tellus, 34:10-16.

Brenner, M. P., Lang, J. H., Li, J., Qiu, J., and Slo, A. H. (2003). Optimal design of a bistable switch. PNAS, 100(17):9663-9667. 
Ferrari, M., Ferrari, V., Guizzetti, M., Andò, B., Baglio, S., and Trigona, C. (2010). Improved energy harvesting from wideband vibrations by nonlinear piezoelectric converters. Sens. Act. A, 162:425-431.

Gammaitoni, L., Hänggi, P., Jung, P., and Marchesoni, F. (1998). Stochastic resonance. Rev. Mod. Phys., 70(1):223-287.

Gammaitoni, L., Marchesoni, F., Menichella-Saetta, E., and Santucci, S. (1989). Stochastic resonance in bistable systems. Phys. Rev. Lett., 62(4):349-352.

Harmer, G. P. and Abbott, D. (2001). Motion detection and stochastic resonance in noisy environments. Microelectr. J., 32:959-967.

He, H.-L., Wang, T.-Y., Leng, Y.-G., Zhang, Y., and Li, Q. (2007). Study on nonlinear filter characteristic and engineering application of cascaded bistable stochastic resonance system. Mech. Syst. Signal Pr., 21:2740-2749.

Holmes, P. J. and Snell, J. E. (1966). A vapour etching technique for the photolithography of silicon dioxide. Microelectron. Reliab., 5(4):337-341.

Jansen, H., de Boer, M., Wiegerink, R., Smulders, E., Neagu, C., and Elwenspoek, M. (1997). RIE lag in high aspect ratio trench etching of silicon. Microelectron. Eng., 35:45-50.

Kaajakari, V., Mattila, T., Oja, A., and Seppä, H. (2004). Nonlinear limits for single-crystal silicon microresonators. J. Microelectromech. S., 13(5):715-724.

Kenfack, A. and Singh, K. P. (2010). Stochastic resonance in coupled underdamped bistable systems. Phys. Rev. E, 82:046224.

Kramers, H. A. (1940). Brownian motion in a field of force and the diffusion model of chemical reactions. Physica, 7(4):284-304.

Krylov, S., Ilic, B. R., Schreiber, D., Serentensky, S., and Craighead, H. (2008). The pull-in behavior of electrostatically actuated bistable microstructures. $J$. Micromech. Microeng., 18:055026.

Kuijpers, A. A., Krijnen, G. J. M., Wiegerink, R. J., Lammerink, T. S. J., and Elwenspoek, M. C. (2006). A micromachined capacitive incremental position sensor. J. Micromech. Microeng., 16:116-134.

Kuipers, W. J., van Baar, J. J., Dijkstra, M., Wiegerink, R. J., Lammerink, T. S. J., de Boer, J. H., and Krijnen, G. J. M. (2006). Drag force actuated bistable microswitches for flow sensing. In IEEE MEMS, pp. 658-661, Istanbul, Turkey. 
Laermer, F. and Schilp, A. (1994). Method of anisotropically etching silicon. Patent. US 5501893.

Legtenberg, R., Groeneveld, A. W., and Elwenspoek, M. (1996). Comb-drive actuators for large displacements. J. Micromech. Microeng., 6:320-329.

Levin, J. E. and Miller, J. P. (1996). Broadband neural encoding in the cricket cercal sensory system enhanced by stochastic resonance. Nature, 380:165-166.

Li, J., X.Chen, Du, Z., Fang, Z., and He, Z. (2013). A new noise-controlled second-order enhanced stochastic resonance method with its application in wind turbine drivetrain fault diagnosis. Renew. Energ., 60:7-19.

McInnes, C. R., Gorman, D. G., and Cartmell, M. P. (2008). Enhanced vibrational energy harvesting using nonlinear stochastic resonance. J. Sound. Vib., 318:655662 .

McNamara, B. and Wiesenfeld, K. (1989). Theory of stochastic resonance. Phys. Rev. A, 39(9):4854-4869.

Ono, T., Yoshida, Y., Jiang, Y.-G., and Esashi, M. (2008). Noise-enhanced sensing of light and magnetic force based on a nonlinear silicon microresonator. Appl. Phys. Express, 1:123001.

Palonpon, A., Amistoso, J., Holdsworth, J., Garcia, W., and Saloma, C. (1998). Measurement of weak transmittances by stochastic resonance. Opt. Lett., 23(18):1480-1482.

Polytec (2005). MSA-400 Micro System Analyzer. Polytec, Waldbronn, Germany.

Qiu, J., Lang, J. H., and Slocum, A. H. (2001). A centrally-clamped parallelbeam bistable MEMS mechanism. In IEEE MEMS, pp. 353-356, Interlaken, Switzerland.

Ray, R. and Sengupta, S. (2006). Stochastic resonance in underdamped, bistable systems. Phys. Lett. A, 353:364-371.

Saif, M. T. A. (2000). On a tunable bistable MEMS - Theory and experiment. J. Microelectromech. S., 9(2):157-170.

Shimozawa, T., Murakami, J., and Kumagai, T. (2003). Cricket wind receptors: thermal noise for the highest sensitivity known. In Sensors and sensing in biology and engineering, pp. 145-159. Springer. 
Sulfridge, M., Saif, T., Miller, N., and Meinhart, M. (2004). Nonlinear dynamic study of a bistable MEMS: model and experiment. J. Microelectromech. S., 13(5):725-731.

Wellens, T., Shatokhin, V., and Buchleitner, A. (2004). Stochastic resonance. Rep. Prog. Phys., 67:45-105.

Xu, Y., Li, J., Feng, J., Zhang, H., Xu, W., and Duan, J. (2013). Lévy noise-induced stochastic resonance in a bistable system. Eur. Phys. J. B, 86:198.

Yamahata, C., Sarajlic, E., Krijnen, G. J. M., and Gijs, M. A. M. (2010). Subnanometer translation of microelectromechanical systems measured by discrete fourier analysis of CCD images. J. Microelectromech. S., 19(5):1273-1275.

Zhang, X.-J. (2001). Stochastic resonance in second-order autonomous systems subjected only to white noise. J. Phys. A: Math. Gen., 34:10859-10868. 


\section{7 \\ Biomimetic accelerometer}

\subsection{Introduction}

In biology, mechanosensors, equipped with differing hair-like structures for signal pick-up, are sensitive to a variety of physical quantities like acceleration, flow, rotational rate, balancing and IR-light (Liu, 2007; Schmitz et al., 2012). As an example, crickets have various types of hair-like receptors for measurement of several environmental quantities. For sensing of low-frequency flows to obtain information about the environment and avoid e.g. predator attacks crickets use filiform hairs, which are situated on the back of the cricket's body on appendices called cerci, and which are able to sense airflows with velocity amplitudes down to $30 \mathrm{\mu m} \mathrm{s}^{-1}$ (Shimozawa et al., 1998; Humphrey et al., 1993) and operate around the energy levels of thermal noise (Shimozawa et al., 2003). Crickets gather also information about their environment by the use of bristle hairs which activate interneurons that respond to tactile stimuli of the cercus and abdomen (Murphey, 1985). Further, crickets have club-shaped sensilla, called clavate hairs, located on their cerci (figure 7.1). These clavate hairs turn out to be sensitive to (gravitational) acceleration, providing the cricket information on its orientation (Murphey, 1981; Sakaguchi and Murphey, 1983). For example, a cricket uses its clavate hairs to

This chapter is based on "A biomimetic accelerometer inspired by the cricket's clavate hair" by H. Droogendijk, M. J. de Boer, R. G. P. Sanders, and G. J. M. Krijnen, submitted to J. R. Soc. Interface, (2014). 


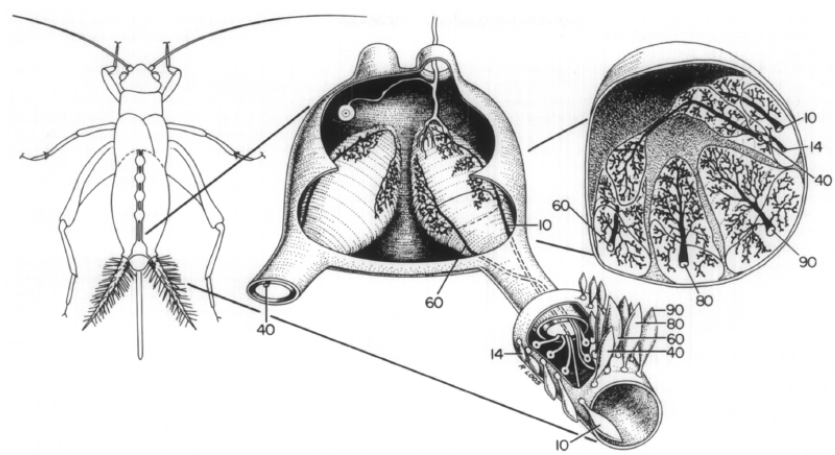

Figure 7.1: Artist's reconstruction of the clavate hair-based sensory system (Murphey, 1981).

compensate head movement when it is rotated around its longitudinal axis (Horn and Bischof, 1983).

For measuring (gravitational) acceleration, numerous types of accelerometers have been realized over the past years using MEMS technology, with applications in e.g. automotive and navigation (Yazdi et al., 1998). Current state-of-the-art commercialized MEMS accelerometers show formidable performance in range, resolution and noise floor. In contrast to the cricket's clavate system, MEMS accelerometers are usually no hair-based systems and frequently contain feedback electronics. To explore some of the intricacies of the clavate hair system and assess its potential to engineering applications, we aim for the design, fabrication and characterization of a biomimetic accelerometer. Biomimetic hair-based structures have been exploited earlier with applications in both actuation and sensing of physical quantities (Zhou and Liu, 2008; Ginsberg et al., 2013), but seldom for inertial measurement. Previously, a hair-like accelerometer has been investigated by Tang et al. (2013), but its response to external accelerations was not demonstrated.

Here, we present the design and fabrication of a biomimetic accelerometer using MEMS technology, and report on its characterization with respect to externally applied accelerations. We also assess, both theoretically and experimentally, responsivity due to flow-induced contributions to the accelerometer's output and derive design guidelines on how to reduce these effects. Further, we address the accelerometer's physical limits with respect to noise levels and discuss the possibility for short-term adaptation of the sensor to the environment. 


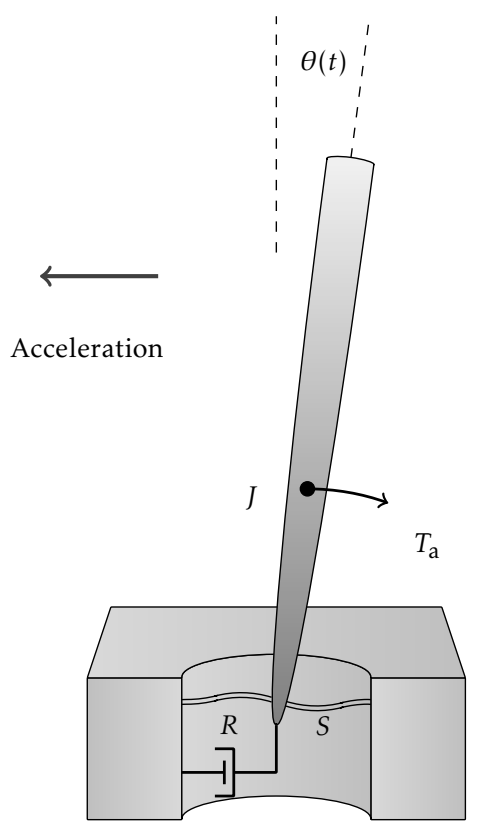

Figure 7.2: Inertial measurement by hair-like structures.

\subsection{Theory and modelling}

\subsubsection{Hair mechanics}

Mechanically, the hair-based accelerometer can be understood as a so-called inverted pendulum which is subjected to external accelerations (figure 7.2). It is described as a second-order rotational-mechanical system with moment of inertia $J$, a rotational stiffness $S$ and a rotational damping $R$, resulting in a description of the system's response to harmonic accelerations by

$$
\theta(\omega)=\frac{T_{\mathrm{a}}}{S-J \omega^{2}+i \omega R},
$$

where for a cylindrical hair, the moment of inertia can be expressed as

$$
J=\frac{\pi \rho d^{2}}{48}\left(4 L^{3}+\frac{3}{4} d^{2} L\right)
$$

In these expressions, $\theta(\omega)$ is the rotational angle amplitude of the hair and $T_{\mathrm{a}}$ is the amplitude of the torque acting on the hair. From (7.2), the moment of inertia $J$ depends strongly on the hair diameter $d$ and hair length $L$. The torque 
$T_{\mathrm{a}}$ is a consequence of external accelerations, or the projections thereof, in the direction perpendicular to the hair and perpendicular to its rotation axis, denoted here by $a_{\text {ext }}$. Using Newton's second law $T_{\mathrm{a}}$ is found by integrating the inertial contributions by over the hair:

$$
T_{\mathrm{a}}=\int_{0}^{L} a_{\mathrm{ext}} \rho \pi\left(\frac{d(z)}{2}\right)^{2} z \mathrm{~d} z=\Xi \cdot a_{\mathrm{ext}},
$$

where $\Xi$ can be expressed as

$$
\Xi=\frac{\pi \eta \rho}{8} d^{2} L^{2}
$$

In these expressions, $\rho$ is the density of the hair and $\eta$ a parameter that depends on the precise geometry of the hair. Further, we assume that the rotational angle amplitudes $\theta$ are small, so the torque $T_{\mathrm{a}}$ can be considered directly proportional with the external acceleration $a_{\text {ext }}$.

\subsubsection{Design}

The hair mechanical system behaves like a classical-second order system, and consequently exhibits the trade-off between responsivity and bandwidth. The responsivity of the hair accelerometer for frequencies well below the system's resonance frequency $\left(\omega \ll \omega_{\mathrm{r}}\right)$ is defined as

$$
\text { Responsivity }=\left.\frac{\mathrm{d} \theta(\omega)}{\mathrm{d} a_{\mathrm{ext}}}\right|_{\omega=0}=\frac{\pi \eta}{8} \frac{\rho d^{2} L^{2}}{S} .
$$

The bandwidth of the system is estimated from the system's resonance frequency:

$$
\text { Bandwidth }=\omega_{\mathrm{r}}=\sqrt{\frac{S}{J}} .
$$

By taking the product of responsivity and bandwidth, a figure of merit (FoM) can be defined for the biomimetic hair accelerometer, similar to the approach described by Krijnen et al. (2007):

$$
\text { FoM }=\text { Responsivity } \times \text { Bandwidth. }
$$

When the hair length is considerably larger than the hair diameter $(L>d)$, this FoM can be simplified to

$$
\mathrm{FoM} \propto \sqrt{\frac{\rho d^{2} L}{S}} .
$$


As a result, to achieve a 'good' hair-based accelerometer, the sensor should have a long and thick hair (high $d$ and $L$ ), as well as a compliant mechanical suspension (low $S$ ).

\subsubsection{Flow contributions}

Although the hair-structure is responsive to external accelerations, such accelerations will lead to a net flow velocity. That is, the hair is moving with a relative velocity $v$ through its surrounding medium, for which we consider the medium velocity itself to be zero:

$$
v=\frac{a_{\mathrm{ext}}}{i \omega}
$$

As a consequence, viscous forces will contribute to the total hair mechanical response for external accelerations. Following the approach given by Shimozawa et al. (1998), these flow-induced contributions can be taken into account. The angular deflection amplitude $\theta_{\mathrm{m}}(\omega)$ for a hair subject to external harmonic acceleration at frequency $\omega$, including flow-induced torque, is found to be:

$$
\theta_{\mathrm{m}}(\omega)=\frac{a_{\mathrm{ext}} \sqrt{\Xi^{2}+\left(\frac{\chi}{\omega}\right)^{2}}}{\sqrt{\left[S-\left(J+J_{\rho}+J_{\mu}\right) \omega^{2}\right]^{2}+\left[\left(R+R_{\mu}\right) \omega\right]^{2}}} .
$$

Here, $\Xi$ is the inertial-induced torque contribution as defined in (7.4) and $\chi$ represents the flow-induced torque contribution (see appendix A):

$$
\chi=\left|Z_{\mathrm{S}}\right| \sqrt{A_{\mathrm{f}}^{2}+B_{\mathrm{f}}^{2}}
$$

where the variables $A_{\mathrm{f}}$ and $B_{\mathrm{f}}$ are defined as

$$
A_{\mathrm{f}}=\int_{0}^{L} \kappa(z) z \cos \left(\zeta_{z}+\eta_{\mathrm{S}}\right) \mathrm{d} z, \quad B_{\mathrm{f}}=\int_{0}^{L} \kappa(z) z \sin \left(\zeta_{z}+\eta_{\mathrm{S}}\right) \mathrm{d} z,
$$

with $\kappa(z)$ a parameter that depends on the flow-profile:

$$
\kappa(z)=\sqrt{1+e^{-2 \beta z}-2 e^{-\beta z} \cos (\beta z)} .
$$

The quantities $J_{\rho}$ and $J_{\mu}$ are often referred to as virtual added mass and $R_{\mu}$ as virtual added damping (Humphrey and Barth, 2008): 
Table 7.1: Experimental values of the biomimetic hair-based accelerometer.

\begin{tabular}{lll}
\hline Quantity & Symbol & Value \\
\hline Hair geometry & & \\
\hline Hair length & $L$ & $\begin{array}{l}800 \mu \mathrm{m} \\
80 \mu \mathrm{m}\end{array}$ \\
Hair diameter & $d$ & \\
\hline Mechanical parameters & \\
\hline Torsional stiffness & $S$ & $9.07 \times 10^{-9} \mathrm{Nm} \mathrm{rad}^{-1}$ \\
Torsional resistance & $R$ & $2.44 \times 10^{-13} \mathrm{Nm} \mathrm{srad}^{-1}$ \\
Moment of inertia & $J$ & $2.25 \times 10^{-15} \mathrm{~kg} \mathrm{~m}^{2}$ \\
\hline Flow contributions $(80 \mathrm{~Hz})$ & \\
\hline Added damping & $R_{\mu}$ & $1.91 \times 10^{-14} \mathrm{Nm} \mathrm{s} \mathrm{rad}^{-1}$ \\
Added mass & $J_{\rho}$ & $1.07 \times 10^{-18} \mathrm{~kg} \mathrm{~m}^{2}$ \\
Added mass & $J_{\mu}$ & $1.94 \times 10^{-17} \mathrm{~kg} \mathrm{~m}^{2}$ \\
\hline
\end{tabular}

$$
J_{\rho}=\frac{\pi \rho d^{2} L^{3}}{12}, \quad J_{\mu}=-\frac{\pi^{2} \mu G L^{3}}{3 g \omega}, \quad R_{\mu}=\frac{4}{3} \pi \mu G L^{3} .
$$

where $\mu$ is the air dynamic viscosity and $\rho$ is the air density. Note that when the flow contributions are small $\left(\chi / \omega \ll \Xi, J_{\rho}+J_{\mu} \ll J\right.$ and $\left.R_{\mu} \ll R\right)$ the expression for the rotational angle $\theta_{\mathrm{m}}(\omega)$ will simplify to (7.1).

To evaluate the analytical model, the values for the parameters of the hair-flow sensory system as given in table 7.1 are used. In this work, given the mechanical parameters $S$ and $R$ listed in table 3.3, the hair geometry ( $L$ and $d$ ) is chosen such that the inertial-induced $T_{\mathrm{a}}$ is large with respect to $T_{\mathrm{f}}$ (generally about $100 \times$ ), so that the hair-based accelerometer is primarily inertially responsive.

\subsubsection{Thermal noise}

An important characteristic of a sensory system is its signal-to-noise ratio (SNR). Generally, when the SNR is equal or higher than one, a system may allow to detect the input signal. Hence, besides the responsivity of the sensor, the noise level of the system determines the fundamental detection threshold of the system. To find the detection threshold of our system, we consider the power spectral density due to the Johnson-Nyquist (thermal) white noise in the system (Gabrielson, 1993): 


$$
\overline{T_{\mathrm{n}}^{2}}=4 k_{\mathrm{B}} T_{0}\left(R+R_{\mu}\right),
$$

where $k_{\mathrm{B}}$ is the Boltzmann's constant, $T_{0}$ is the ambient temperature and $\bar{T}_{n}^{2}$ the square of the noise induced equivalent torque per unit of bandwidth. The mechanical transfer function $H(\omega)$ of the system determines the resulting mechanical response to the noise:

$$
H(\omega)=\frac{1}{S-\left(J+J_{\rho}+J_{\mu}\right) \omega^{2}+j\left(R+R_{\mu}\right) \omega} .
$$

The equivalent noise angle $\Theta_{n}$ is found by integrating the noise power over the full spectrum and taking the square root:

$$
\Theta_{\mathrm{n}}=\sqrt{\int_{0}^{\infty} \overline{T_{\mathrm{n}}^{2}}|H(\omega)|^{2} \mathrm{~d} \omega .}
$$

The detection limit $a_{\mathrm{th}}$ is found by dividing the sensor's equivalent noise angle $\Theta_{\mathrm{n}}$ by the rotational angle $\Theta_{\mathrm{m}}$ for an acceleration of $1 \mathrm{~m} \mathrm{~s}^{-2}$ :

$$
a_{\mathrm{th}}=\frac{\Theta_{\mathrm{n}}}{\left.\Theta_{\mathrm{m}}\right|_{a_{\mathrm{ext}}=1 \mathrm{~ms}^{-2}}} .
$$

Thermal noise calculations for our hair mechanical system indicate a detection threshold for acceleration sensing of about $1.1 \times 10^{-3} \mathrm{~m} \mathrm{~s}^{-2}$ for frequencies within a bandwidth of $1 \mathrm{kHz}$.

\subsection{Fabrication}

The fabrication process for the biomimetic accelerometer is based upon the process for cricket-inspired biomimetic hair flow sensors, previously developed in our group (Bruinink et al., 2009; Dagamseh et al., 2011) A schematic overview of the biomimetic accelerometer with the materials indicated is shown in figure 7.3.

The sensor is fabricated on a silicon-on-insulator wafer. Trenches are etched in the silicon device layer using DRIE. A layer of $200 \mathrm{~nm}$ stoichiometric $\mathrm{Si}_{3} \mathrm{~N}_{4}$ is used for covering and protecting the trenches. The device layer contains two electrodes, which are used for capacitive readout of the acceleration-induced movement. On top of the $\mathrm{Si}_{3} \mathrm{~N}_{4}$ layer, a sacrificial layer of poly-silicon $(1.5 \mu \mathrm{m})$ is deposited by LPCVD. The sensor membrane and springs are constructed by depositing and patterning a $1 \mu \mathrm{m}$ SiRN layer on top of the poly-silicon. Aluminium $(80 \mathrm{~nm})$ is sputtered on top of the membrane to create the electrodes for capacitive read-out. 


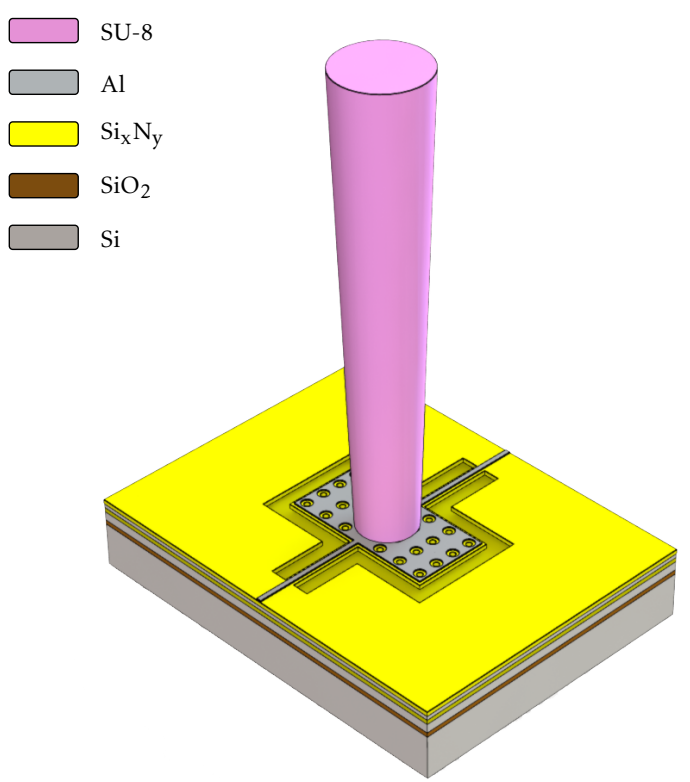

Figure 7.3: Schematic view of the MEMS hair-based accelerometer.

Our artificial clavate hair is created by two layers of SU-8, to realize both the centre of mass towards the top of the hair structure and a total hair length of about $800 \mu \mathrm{m}$ with an average diameter of about $80 \mu \mathrm{m}$. Finally, to release the membrane the sacrificial poly-silicon layer is removed using $\mathrm{XeF}_{2}$ etching. The fabrications results are shown by the SEM image in figure 7.4.

\subsection{Experimental}

\subsubsection{Setup}

Experiments to measure the sensor's response are performed using the setup shown in figure 7.5. A waveform generator (Agilent 33220A-001) is used to produce a sinusoidal signal at a frequency $f(\omega=2 \pi f)$ that is supplied to an amplifier. This amplifier drives a voice-coil shaker (MB Electronics PM 50) to generate harmonic acceleration. Carrier signals at $1 \mathrm{MHz}$ are supplied to the bottom electrodes of the accelerometer for capacitive read-out. The top electrode is connected to a charge amplifier, and after demodulation and filtering (Stanford SR 650) the sensor's response is monitored on an oscilloscope (Agilent DS1024). For calibration, the applied accelerations were measured by mounting a reference accelerometer (STEVAL-MKI021V1) on the shaker. 


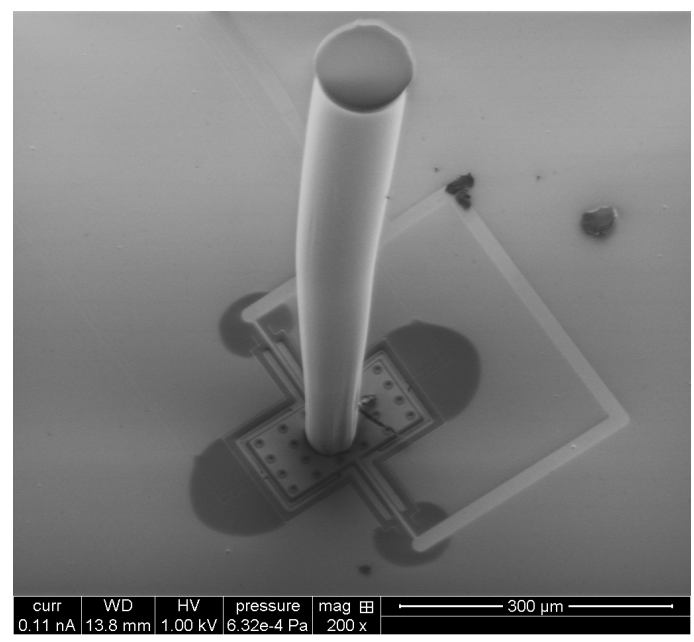

Figure 7.4: MEMS hair-based accelerometer fabricated by surface micromachining and using SU-8 lithography.

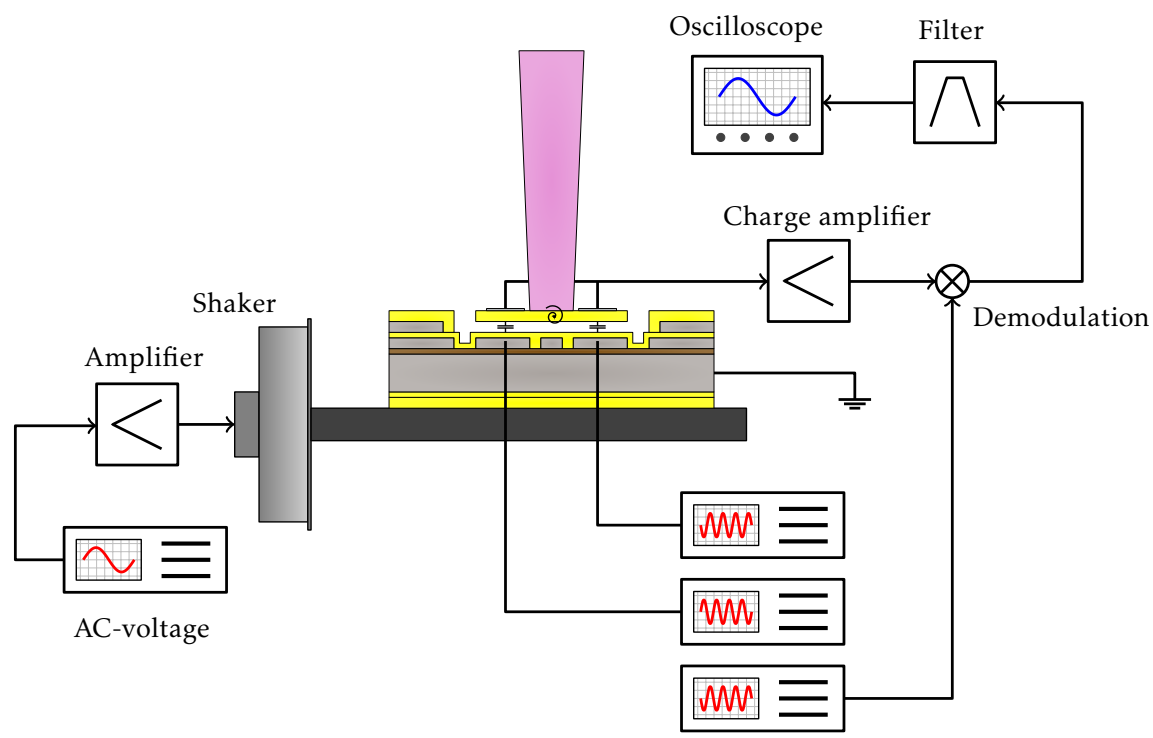

Carrier signals

Figure 7.5: Capacitive measurement setup for determining the membrane rotation of the biomimetic accelerometer. 


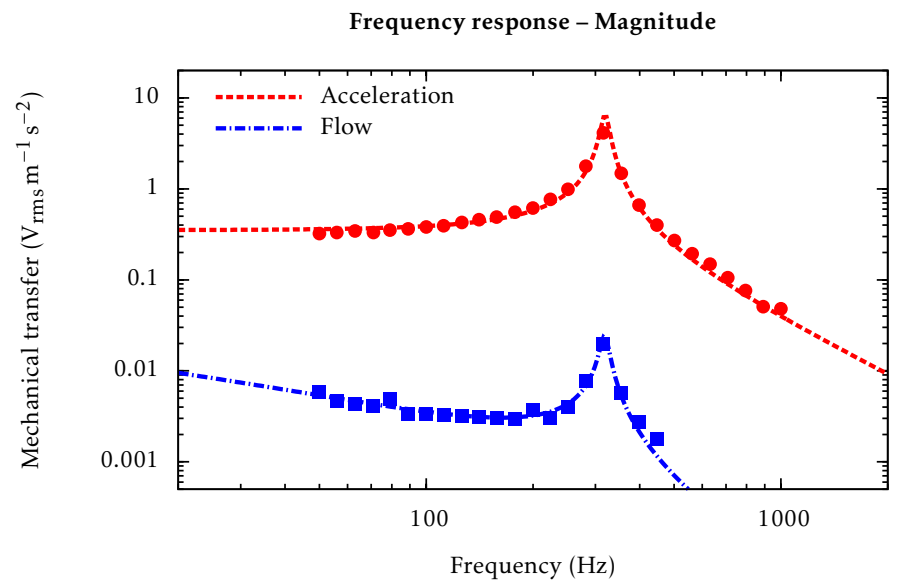

Figure 7.6: Measured mechanical transfer of the hair-based accelerometer using capacitive read-out.

\subsubsection{Frequency response}

First, the frequency response of the hair-based accelerometer was measured using capacitive read-out in the direction perpendicular to the rotational axis. Frequencies within a range of $50-1000 \mathrm{~Hz}$ were applied to the shaker. The reference accelerometer was used to determine the externally applied acceleration amplitude. The resulting measured magnitude response of the biomimetic accelerometer is shown in red in figure 7.6. Here, the circles represent the measurements and the dashed line exhibits the analytical model based on (7.1), where the resonance frequency $\omega_{0}$ and the quality factor $Q$ were fitted. We observe good agreement between model and measurements, where the resonance frequency is found to be about $320 \mathrm{~Hz}$.

Additionally, flow measurements have been performed by replacing the shaker by a loudspeaker to apply an oscillating airflow. The measured response for accelerations induced flow is shown in blue in figure 7.6, where the measured flow response per $\mathrm{m} \mathrm{s}^{-1}$ has been normalised to acceleration equivalent flow response using (7.9). Here, the squares represent the measurements and the dash-dotted line exhibits the analytical model based on (7.10) with $\Xi=0$. Again, good agreement between model and measurements is observed, with a similar resonance behaviour. Furthermore, the overall responsivity to acceleration induced flow is significantly lower than the responsivity to acceleration, as desired.

In addition to the magnitude of the response of the hair-based accelerometer, also the phase of its response was measured. Both measurements (points) and 


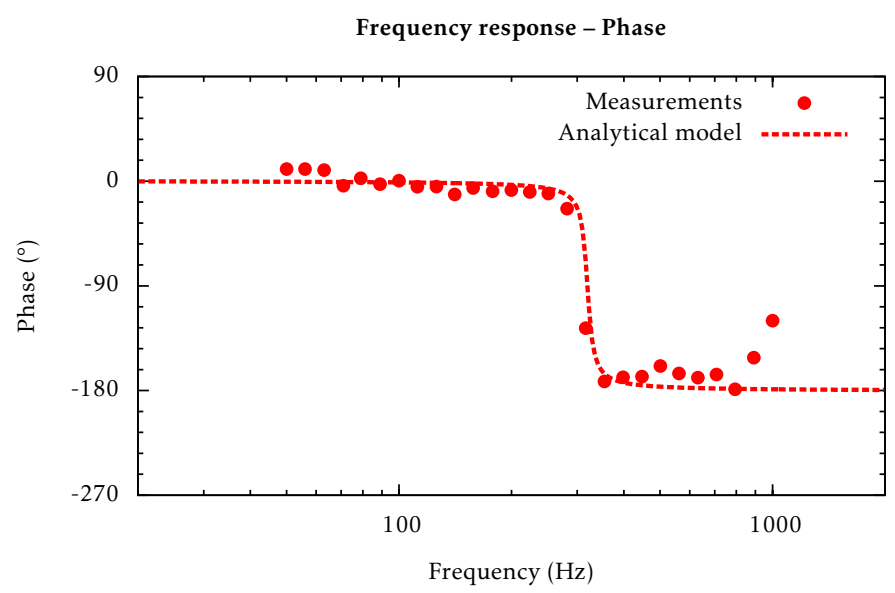

Figure 7.7: Measured phase-shift of the hair-based accelerometer using capacitive read-out.

analytical model (dashed line) are shown in figure 7.7. We observe good agreement between measurements and model, and the phase-shift of about $180^{\circ}$ around resonance is clearly visible.

\subsubsection{Directivity}

The sensor's directivity was measured by rotating it over $360^{\circ}$, with steps of $10^{\circ}$, with respect to the direction of the applied external acceleration, while using capacitive read-out. To this end harmonic acceleration with a frequency of $80 \mathrm{~Hz}$ was applied and the output voltage was measured by a multimeter (Keithley 2000). The obtained results are shown in figure 7.8. We observe that the measurements are in close agreement with the theoretical response for a so-called figure-of-eight. The measurements indicate that the hair-based accelerometer has a maximum responsivity for both $0^{\circ}$ and $180^{\circ}$, which coincides with the direction perpendicular to the rotational axis of the hair sensor.

\subsubsection{Threshold and linearity}

To obtain a model which describes the sensor's signal to noise ratio (SNR) as a function of acceleration amplitude as well as the sensor's detection threshold, the signal and noise powers are considered. The signal is assumed to have a linear relationship with respect to the acceleration amplitude $a_{0}$, given by the coefficient $S_{\mathrm{c}}(\omega)$. This coefficient is directly related to the sensor's rotational angle $\theta(\omega)$ and therefore has a dependency on the acceleration frequency $\omega . N_{c}$ is a 


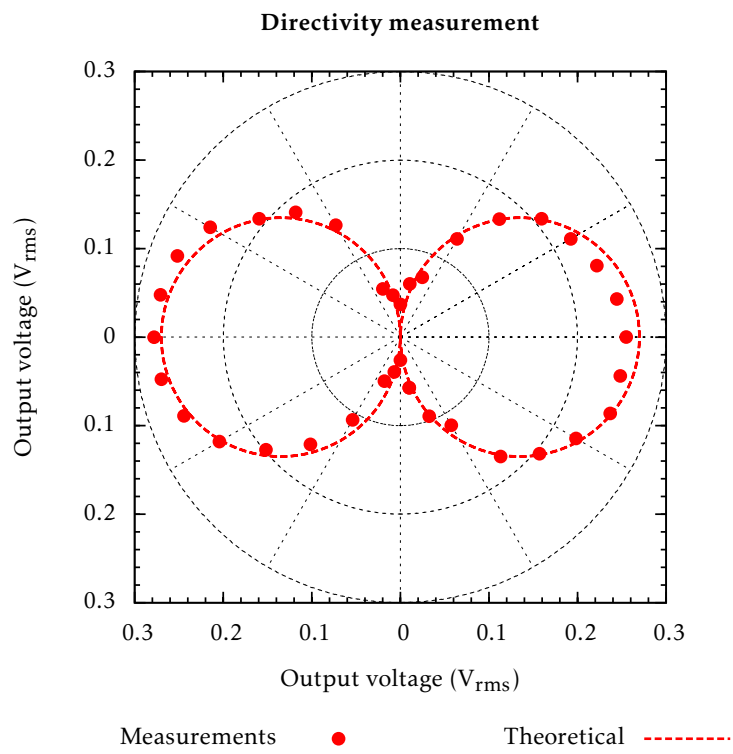

Figure 7.8: Measured directivity of the hair-based accelerometer using capacitive read-out at an acceleration frequency of $80 \mathrm{~Hz}$.

constant representing the total noise, giving for the measured output $z$ due to the uncorrelated sources:

$$
z(\omega)=\sqrt{\left(S_{\mathrm{c}}(\omega) a_{\mathrm{ext}}\right)^{2}+N_{\mathrm{c}}^{2}}
$$

From this expression, the measured detection threshold $a_{\min }$ can be found by equating the noise and signal powers (or alternatively by calculating the intersection value of the asymptotes) which requires only the values for $S_{\mathrm{c}}$ and $N_{\mathrm{c}}$ :

$$
a_{\min }(\omega)=\frac{N_{\mathrm{c}}}{S_{\mathrm{c}}(\omega)} .
$$

Experiments to determine the sensor's linearity were performed by choosing first a specific acceleration frequency $(80 \mathrm{~Hz})$ and then by varying the acceleration amplitude. Subsequently, from the measured output rms-voltage the sensor's detection limit and linearity are derived. The results are shown in figure 7.9, where the points represent the measurements, the solid line is based on (7.19), and the dashed lines indicate the constant equivalent noise amplitude and ideal linear response asymptotes. We observe that for accelerations with an amplitude of more 


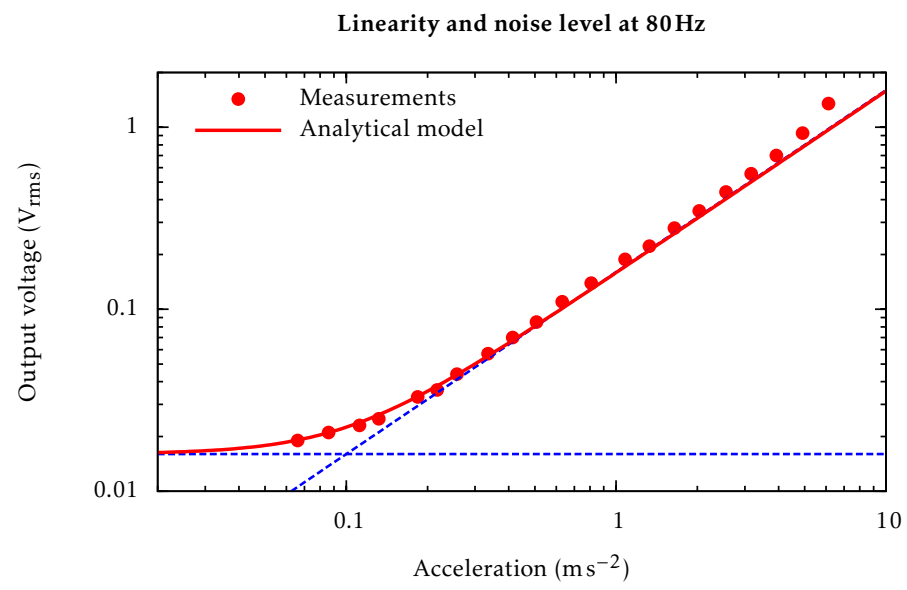

Figure 7.9: Measured response versus acceleration amplitudes at a frequency of $80 \mathrm{~Hz}$ using capacitive read-out.

than $0.1 \mathrm{~m} \mathrm{~s}^{-2}$, indicated by the intersection of the asymptotes, the hair-based accelerometer exhibits a clear linear relationship with the applied acceleration. Below this amplitude, the sensor's output is dominated by noise $(\mathrm{SNR}<1)$.

To get some insight in the accelerometer's noise performance and stability, an Allan variance measurement was performed. The zero-acceleration output rms-voltage was measured with a time interval of $20 \mathrm{~ms}$ for a period of $2 \mathrm{~h}$ using a multimeter (Agilent 34401A) connected to LabVIEW. The results of the subsequently calculated Allan deviation are shown in figure 7.10, together with asymptotic lines for both the velocity random walk and the bias instability.

From the linearity measurements, the full-scale error was calculated and found to be $3.3 \%$. By considering the detection threshold and the full scale of accelerations, the dynamic range of the hair-based accelerometer is about $35.6 \mathrm{~dB}$. The Allan variance results showed a velocity random walk of $1.67 \mathrm{~m} \mathrm{~s}^{-1} \sqrt{\mathrm{h}}^{-1}$ and a bias instability of $18 \mathrm{~m} \mathrm{~s}^{-1} \mathrm{~h}^{-1}$. To summarize, an overview of the sensor performance is shown in table 7.2.

\subsection{Discussion}

The measured threshold of the biomimetic accelerometer is about $0.10 \mathrm{~m} \mathrm{~s}^{-2}$, which is about a factor 100 larger than the calculated thermal noise based value of $1.1 \times 10^{-3} \mathrm{~m} \mathrm{~s}^{-2}$. The relative large detection threshold is due to the presence of comparatively strong noise sources in the read-out electronics. Improvement of 


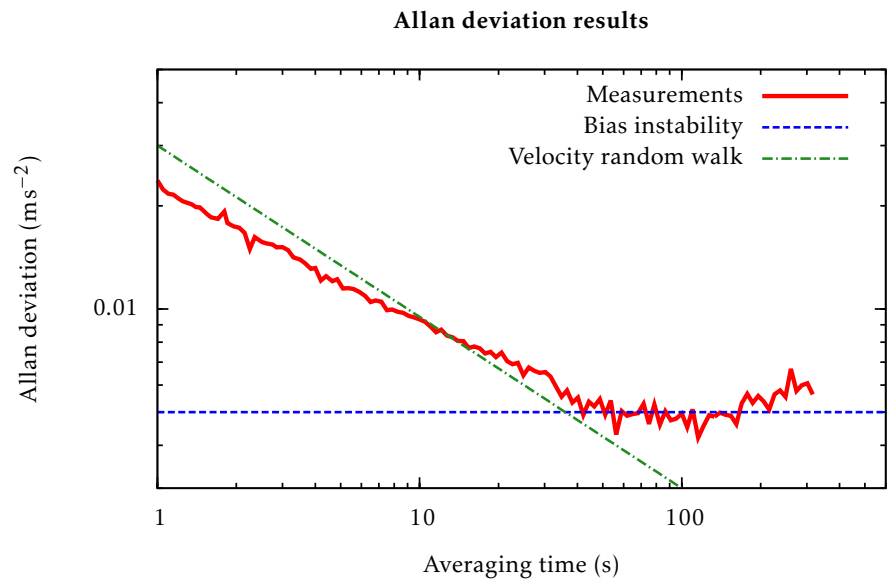

Figure 7.10: Measured Allan deviation using capacitive read-out.

Table 7.2: Experimental values of the biomimetic hair-based accelerometer.

\begin{tabular}{lll}
\hline Quantity & Symbol & Value \\
\hline Mechanical parameters & & \\
\hline Quality factor & $Q$ & 12.5 \\
Resonance frequency & $f_{0}$ & $319.6 \mathrm{~Hz}$ \\
\hline Sensor performance $(80 \mathrm{~Hz})$ & \\
\hline Threshold & $a_{\mathrm{min}}$ & $0.10 \mathrm{~m} \mathrm{~s}^{-2}$ \\
Dynamic range & $\mathrm{DR}$ & $\geq 35.6 \mathrm{~dB}$ \\
Full scale error & $\epsilon_{\mathrm{FS}}$ & $3.3 \%$ \\
Velocity random walk & $\mathrm{VRW}$ & $1.67 \mathrm{~m} \mathrm{~s}^{-1} \sqrt{\mathrm{h}}-1$ \\
Bias instability & $\sigma_{\mathrm{b}}$ & $5 \times 10^{-3} \mathrm{~m} \mathrm{~s}^{-2}$ \\
Thermal noise limit & $a_{\mathrm{th}}$ & $1.1 \times 10^{-3} \mathrm{~m} \mathrm{~s}^{-2}$ \\
\hline
\end{tabular}

these electronics, by reduction of its noise, will help to bring the detection threshold for the accelerometer towards the thermal noise limited threshold. Similar differences between measured and (calculated) thermal noise levels have been observed for our previously reported biomimetic hair flow sensors (Droogendijk et al., 2012; Dagamseh et al., 2013).

As stated in section 7.2, the hair sensor is designed in such a way to have a negligible flow-induced response. Therefore, the ratio between responsivity to acceleration and responsivity to acceleration-induced flow is desired to be large. 


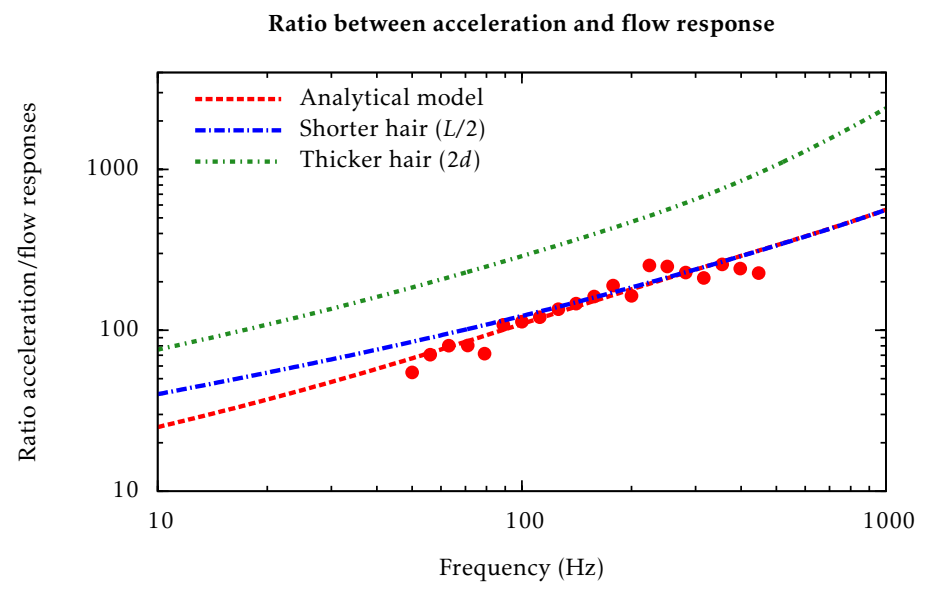

Figure 7.11: Optimization of hair geometry for improvement of the ratio of the acceleration and flow ratio responses.

This ratio is shown in figure 7.11, together with its dependence on $L$ and $d$. As stated earlier, the hair length $L$ and diameter $d$ have a large impact on both inertial and flow-induced sensing, and can be used to optimize the sensor with respect to its flow responsivity. In figure 7.11 , the dashed red line indicates the original response ratio, where the measurements based upon data from figure 7.6 are represented by circles. Although the hair length $L$ has significant impact on both flow and inertial responsivity, only a slight improvement is gained in response ratio for lower frequencies when shortening the hair (dash-dotted blue line). However, by maintaining the hair length and increasing the thickness of the hair by a factor 2 , an improvement of more than $300 \%$ can be gained for all frequencies within the sensor's bandwidth (dotted green line). Also, by considering the figure of merit for a hair flow sensor (Krijnen et al., 2007), the $\mathrm{FoM}_{\text {flow }}$ scales with $d^{1 / 3}$, while the FoM $\mathrm{Mcc}_{\mathrm{acc}}$ of the accelerometer scales with $d^{2}$ following (7.8). Therefore, to realize a 'good' accelerometer the hair diameter is important for having both a good figure of merit and minimal flow responsivity.

As stated in the section on theory and modelling, the rotational angle amplitudes $\theta$ are assumed to be small. However, the presence of the Earth's gravitational field will lead to a projection of the acceleration $g$ in the direction of movement of the hair when operating in the horizontal plane, comparable to a pendulum. As a consequence, the torque exerted on the hair due to $g$ can be calculated using (7.3), which leads to an additional angle-dependent term and can be regarded as a contribution to the system's torsional stiffness. Consequently, the system's 
frequency response changes to:

$$
\theta(\omega)=\frac{T_{\mathrm{a}}}{\left[S-S_{\mathrm{g}}\right]-J \omega^{2}+i \omega R},
$$

where $S_{\mathrm{g}}$ is the contribution due to Earth's gravitational acceleration:

$$
S_{\mathrm{g}}=\eta \frac{\pi \rho}{8} d^{2} L^{2} g
$$

In our case, this contribution due to $g$ is negligible, since $S_{\mathrm{g}}$ has a value of $1.88 \times 10^{-11} \mathrm{Nm} \mathrm{rad}^{-1}$ in case of a straight hair $(\eta=1)$, and the value of the system's mechanical torsional stiffness is about three orders of magnitude larger.

Generally, the susceptibility for (gravitational) acceleration is used by crickets for determination of their position and orientation. The hair-based accelerometer described in this work allows in principle also for determination of orientation using the Earth's gravitational field. That is, by measuring the projection of the Earth's gravitational acceleration, the angle of rotation of the accelerometer with respect to Earth can be determined. However, since the fabricated accelerometer has limits with respect to resolution, an error in this angle will result. Based on the experimental data listed in table 6.1, this error is calculated to be in the order of $0.7^{\circ}$ for accelerations well below resonance $(\omega \rightarrow 0)$, which emphasizes the potential use of this accelerometer to determine orientation.

As we have shown in figure 7.8, the hair-based accelerometer has a strong directivity. In our MEMS version this directivity stems from both the mechanical design, which primarily allows rotation around the torsional axis of the sensor, and the differential capacitive read-out, which causes a strong reduction of signals caused by tilting of the hair. As a consequence multiple hair-based accelerometers may be used simultaneously to sense acceleration in 3D. In crickets filiform hairs have been shown to have preferential directions of rotations with ratios in stiffness of 'hard' over 'easy' directions between 4 and 8 (Kanou et al., 1989). If such directivity also exists in the clavate hairs is to our knowledge unknown. Nevertheless, Sakaguchi and Murphey (1983) have shown that crickets use the many clavate hair-sensors on their cerci for determination of their orientation relative to the gravitational field and that they do so both with respect to roll (rotation around longitudinal axis of the animal) and pitch.

Keeping inspired by biology, the performance of a sensory system can be adapted to the environment. For example, the bullfrog's internal ear displays adaptation by mechanical relaxation of hair bundles (Howard and Hudspeth, 1987), leading to a change in stiffness of the saccular hair cell. A similar technique can be applied to the biomimetic accelerometer by electrostatically reducing the 
torsional stiffness $S$, and could be useful for e.g. robotic applications in which adaptation to the environment is required. The technique of electrostatic spring softening (ESS) has been investigated thoroughly for our biomimetic hair-based flow sensors, and can lead to an improvement in responsivity of more than $80 \%$ and a reduced sensory threshold of more than $50 \%$ in case of noise which is dominated by e.g. electronics (Droogendijk et al., 2012). Since the accelerometer's geometry and parameters are similar to those of the hair flow sensor, comparable results can be expected.

\subsection{Conclusions}

A biomimetic accelerometer has been developed and fabricated using surface micromachining and SU-8 lithography, inspired by the clavate hair system of the cricket. We showed that this MEMS hair-based accelerometer has a resonance frequency of $320 \mathrm{~Hz}$, a detection threshold of $0.10 \mathrm{~m} \mathrm{~s}^{-2}$ and a dynamic range of more than $35 \mathrm{~dB}$. Further, the accelerometer has low responsivity to airflow, clear directivity and a bias instability of $5 \times 10^{-3} \mathrm{~ms}^{-2}$. We have argued that the accelerometer can be further optimized by increasing the hair diameter, and that it principally allows for adaptation to the environment by exploiting the mechanism of ESS.

\section{References}

Bruinink, C. M., Jaganatharaja, R. K., de Boer, M. J., Berenschot, J. W., Kolster, M. L., Lammerink, T. S. J., Wiegerink, R. J., and Krijnen, G. J. M. (2009). Advancements in technology and design of biomimetic flow-sensor arrays. In Proc. MEMS 2009, pp. 152-155.

Dagamseh, A., Lammerink, T., Sanders, R., Wiegerink, R., and Krijnen, G. (2011). Towards high-resolution flow cameras made of artificial hair flow-sensors for flow pattern recognition. In Proc. MEMS 2011, pp. 648-651, Cancun, Mexico.

Dagamseh, A. M. K., Bruinink, C. M., Wiegerink, R. J., Lammerink, T. S. J., Droogendijk, H., and Krijnen, G. J. M. (2013). Interfacing of differentialcapacitive biomimetic hair flow-sensors for optimal sensitivity. J. Micromech. Microeng., 23:035010.

Droogendijk, H., Bruinink, C. M., Sanders, R. G. P., Dagamseh, A. M. K., Wiegerink, R. J., and Krijnen, G. J. M. (2012). Improving the performance of biomimetic 
hair-flow sensors by electrostatic spring softening. J. Micromech. Microeng., 22(6):065026.

Gabrielson, T. B. (1993). Mechanical thermal noise in micromachined acoustic and vibration sensors. IEEE Trans. Electron. Devices, 40:903-909.

Ginsberg, M., Schiano, J., Kramer, M., and Alleyne, M. (2013). A case study in bio-inspired engineering design: defense applications of exoskeletal sensors. Defense E Security Analysis, 29(2):156-169.

Horn, E. and Bischof, H.-J. (1983). Gravity reception in crickets: The influence of cereal and antennal afferences on the head position. J. Comp. Physiol. A, 150:93-98.

Howard, J. and Hudspeth, A. J. (1987). Mechanical relaxation of the hair bundle mediates adaptation in mechanoelectrical transduction by the bullfrog's saccular hair cell. PNAS, 84:3064-3068.

Humphrey, J., Devarakonda, R., Iglesias, I., and Barth, F. (1993). Dynamics of arthropod filiform hairs. I. Mathematical modeling of the hair and air motions. Phil. Trans.: Bio. Sc., 340:423-444.

Humphrey, J. A. C. and Barth, F. (2008). Medium flow-sensing hairs: biomechanics and models. Adv. Insect Phys., 34:1-80.

Kanou, M., Osawa, T., and Shimozawa, T. (1989). Mechanical polarization in the air-current sensory hair of a cricket. Experientia, 45:1082-1083.

Krijnen, G., Floris, A., Dijkstra, M., Lammerink, T., and Wiegerink, R. (2007). Biomimetic micromechanical adaptive flow-sensor arrays. Proc. SPIE, 6592:65920F.

Liu, C. (2007). Micromachined biomimetic artificial haircell sensors. Bioinsp. Biomim., 2:162-169.

Murphey, R. K. (1981). The structure and development of a somatotopic map in crickets: The cereal afferent projection. Dev. Biol., 88:236-246.

Murphey, R. K. (1985). A second cricket cercal sensory system: bristle hairs and the interneurons they activate. J. Comp. Physiol. A, 156:357-367.

Sakaguchi, D. S. and Murphey, R. K. (1983). The equilibrium detecting system of the cricket: physiology and morphology of an identified interneuron. J. Comp. Physiol. A, 150:141-152. 
Schmitz, H., Soltner, H., and Bousack, H. (2012). Biomimetic infrared sensors based on photo-mechanic infrared receptors in pyrophilous ("fire-loving") insects. IEEE Sensors J., 12(2):281-288.

Shimozawa, T., Kumagai, T., and Baba, Y. (1998). Structural scaling and functional design of the cercal wind-receptor hairs of cricket. J. Comp. Physiol. A, 183:171186.

Shimozawa, T., Murakami, J., and Kumagai, T. (2003). Cricket wind receptors: thermal noise for the highest sensitivity known. In Sensors and sensing in biology and engineering, pp. 145-159. Springer.

Tang, Y., Peterson, R. L., and Najafi, K. (2013). Technology for fabricating dense 3-D microstructure arrays for biomimetic hair-like sensors. In Proc. MEMS 2013, pp. 355-358, Taipei, Taiwan.

Yazdi, N., Ayazi, F., and Najafi, K. (1998). Micromachined inertial sensors. Proc. IEEE, 86(8):1640-1659.

Zhou, Z.-G. and Liu, Z.-W. (2008). Biomimetic cilia based on MEMS technology. J. Bion. Eng., 5:358-365. 


\section{8 \\ Biomimetic gyroscope}

\subsection{Introduction}

In biology, insects regularly use hair-based structures for measurement of physical quantities like acceleration, flow, rotational rate and equilibrium (Liu, 2007). For example, a cricket uses club-shaped (clavate) hairs to compensate head movement when it is rotated around its longitudinal axis (Horn and Bischof, 1983). Also, bristle hairs are found on the cricket, which are shown to be sensitive to tactile stimuli. Furthermore, filiform hairs are present at the cricket's cerci for measurement of low-frequency airflows (Tobias and Murphey, 1979; Shimozawa and Kanou, 1984).

Another example of a biological hair-based sensory system is the fly's haltere structure. Halteres are tiny club-shaped organs that beat in anti-phase to the wings during flight (Fraenkel and Pringle, 1938), and function as gyroscopes by measuring the flies body rotation using Coriolis forces (Pringle, 1948). The rotation-dependent position of the haltere (figure 8.1) is measured in sensitive strain fields (Hengstenberg, 1998). It has been demonstrated that this halterebased system is used for balancing, guidance and flight stability (Sandeman and Markl, 1980; Nalbach and Hengstenberg, 1994; Chan et al., 1998; Ristroph et al.,

This chapter is based on "Towards a biomimetic gyroscope inspired by the fly's haltere using MEMS technology" by H. Droogendijk, R. A. Brookhuis, M. J. de Boer, R. G. P. Sanders, and G. J. M. Krijnen, submitted to J. R. Soc. Interface, (2014). 


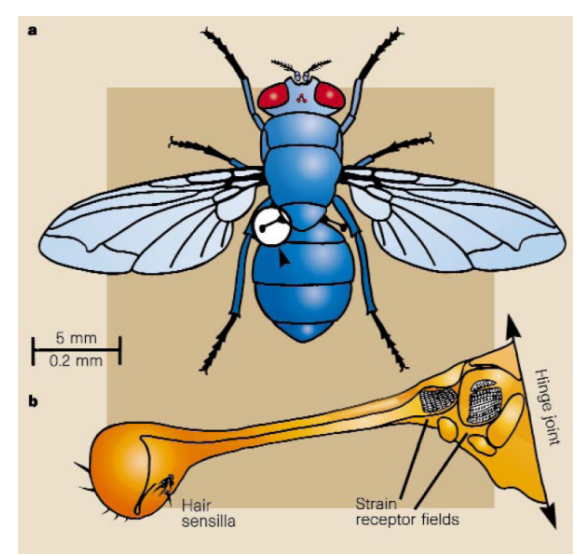

Figure 8.1: Halteres of the blowfly Calliphora vicina. During walking and flight, the halteres oscillate in a vertical plane around a proximal hinge (Hengstenberg, 1998).

2010).

For measuring angular rate, numerous types of gyroscopes ${ }^{1}$ have been realized over the past years using MEMS technology, where current state-of-the-art MEMS gyroscopes show formidable performance in range, resolution and noise floor (Yazdi et al., 1998; Xie and Fedder, 2003; Alper and Akin, 2005; Trusov et al., 2011). In contrast to the fly's haltere system, MEMS gyroscopes are typically no hair-based systems, are generally heavily underdamped and operate at rather high frequencies. In contrast, the fly's haltere system is a close to critically damped system and operates around the fly's wing beat frequency $(130-150 \mathrm{~Hz})(\mathrm{Nalbach}$ and Hengstenberg, 1994). Obviously, the fly's haltere system has developed under evolutionary pressure and therefore addresses the sensory needs of the fly. As a result, not only the operation principle is different from those generally employed in MEMS gyroscopes but also the performance may score high on metrics other than normally used for MEMS gyroscopes. For example bandwidth and response time may be far more essential for flies than (bias-) stability and noise floor, as the halteres have been proven to aid in flight dynamics of flies with fast $(60 \mathrm{~ms})$ responses to flight disturbances (Ristroph et al., 2010).

Previous research on bio-inspired gyroscopic systems (using foils of stainless steel) shows advantages on using a haltere-based gyroscope by achieving a higher sensitivity and a lower power consumption compared to conventional MEMS gyroscopes (Wu et al., 2002; Wu and Wood, 2006). Also Tang et al. (1997) and Challoner et al. (2007) fabricated hair-based gyroscopes using MEMS technology,

\footnotetext{
${ }^{1}$ In this work, all gyroscopes are so-called Coriolis vibratory gyroscopes, which are in fact angular rate sensors. By integration of the measured angular rate, information about the angle is obtained.
} 
although these devices had still macroscopic dimensions compared to the fly's haltere and were severely underdamped by operating them in vacuum. Wicaksono et al. (2007) discuss the preliminary design and modelling of a bio-inspired MEMS gyroscope with electrostatic driving and piezo-resistive read-out. Smith et al. (2012) report on the design and fabrication of a MEMS haltere to use as a sensor in a micro-autonomous system.

In this chapter, we aim for the design, fabrication and optimization of a biomimetic gyroscope using MEMS technology and haltere-like dimensions. Doing so, we intend to investigate some of the intricacies of the haltere system and assess its usability with respect to engineering applications by considering haltere dimensions comparable to the fly's haltere.

\subsection{Theory and modelling}

\subsubsection{Mechanics}

The dynamics of the haltere-based gyroscope and common MEMS gyroscopes are similar, since both types consist of vibrating structures governed by two coupled second order differential equations. The design of these gyroscopes is in general a 2-D isotropic, damped mass-spring system. To describe the haltere mechanics, we first define the force $\vec{F}$ acting on the haltere knob (Nalbach, 1993):

$$
\vec{F}=m \vec{g}-m \ddot{\overrightarrow{r_{\mathrm{i}}}}-m \overrightarrow{a_{\mathrm{f}}}-m \dot{\vec{\Omega}} \times \overrightarrow{r_{\mathrm{i}}}-m \vec{\Omega} \times\left(\vec{\Omega} \times \overrightarrow{r_{\mathrm{i}}}\right)-2 m \vec{\Omega} \times \dot{\overrightarrow{r_{\mathrm{i}}}},
$$

where $\times$ denotes the vector product, $m$ is the mass of the knob, $\vec{g}$ is the gravitational acceleration, $\vec{r}_{\mathrm{i}}$ is the position of the knob with respect to the fly, $\vec{a}_{\mathrm{f}}$ is the linear acceleration of the fly in space, and $\vec{\Omega}$ is the angular rate. Six forces contribute to the total inertial force: gravitational $(m \vec{g})$, primary $(m \ddot{\vec{r}})$, linear acceleration $(m \vec{a})$, angular acceleration $\left(m \dot{\vec{\Omega}} \times \vec{r}_{\mathrm{i}}\right)$, centrifugal $\left(m \vec{\Omega} \times\left(\vec{\Omega} \times \overrightarrow{r_{\mathrm{i}}}\right)\right)$ and Coriolis $\left(2 m \vec{\Omega} \times \dot{\overrightarrow{r_{\mathrm{i}}}}\right)$.

Now, we assume that linear accelerations are absent $\left(\overrightarrow{a_{\mathrm{f}}}=0\right)$, gravitational acceleration $\vec{g}$ is only present in the $z$-direction (the axial direction of the haltere), angular rates are applied only in the horizontal $x y$-plane $\left(\Omega_{x}=\Omega_{y}=0\right)$, the movement of the haltere is harmonic with frequency $\omega$ and the angular rate $\Omega$ is harmonic with frequency $v$ and amplitude $\hat{\Omega}$ :

$$
\Omega=\hat{\Omega} \cos (v t)=\frac{1}{2} \hat{\Omega}\left(e^{j v t}+e^{-j v t}\right) .
$$

Consequently, the force amplitude $\vec{F}$ for the $x$ and $y$-direction, since the $z$-direction is not of interest to us, is given by: 


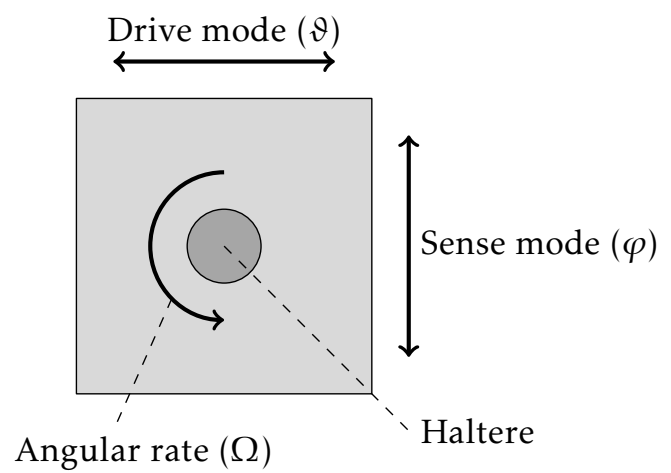

Figure 8.2: Operation principle of the biomimetic gyroscope (top view).

$$
\hat{\vec{F}}=m\left[\begin{array}{cc}
\omega^{2}+\frac{1}{2} \hat{\Omega}^{2}+\frac{1}{2} \hat{\Omega}^{2} \cos (2 v t) & -j 2 \omega \hat{\Omega} \cos (v t)+j v \hat{\Omega} \sin (v t) \\
j 2 \omega \hat{\Omega} \cos (v t)-j v \hat{\Omega} \sin (v t) & \omega^{2}+\frac{1}{2} \hat{\Omega}^{2}+\frac{1}{2} \hat{\Omega}^{2} \cos (2 v t)
\end{array}\right]\left[\begin{array}{c}
\hat{x} \\
\hat{y}
\end{array}\right],
$$

where $\hat{x}$ and $\hat{y}$ denote the amplitudes in the $x$ and $y$-direction respectively. Now, the coordinate system is changed into cylindrical coordinates, as shown in figure 8.2. The system is continuously driven in a steady-state rotational motion in the drive mode with angle $\vartheta$. Rotation around the haltere induces Coriolis-based motion in the sense mode, with the angle $\varphi$ proportional to the input angular rate $\Omega$. By using the following relationships, the force acting on the haltere can be written as a torque $\vec{T}$ for small angle amplitudes $\hat{\vartheta}$ and $\hat{\varphi}$ :

$$
J=\eta m L^{2}, \quad \hat{x}=L \sin (\hat{\vartheta}) \approx L \hat{\vartheta}, \quad \hat{y}=L \sin (\hat{\varphi}) \approx L \hat{\varphi},
$$

where $L$ is the haltere length, $J$ is the moment of inertia and $\eta$ is a parameter depending on the precise geometry. Here, $\eta=1$ by considering the haltere knob as a point mass. As a result, the torque acting on the haltere becomes

$$
\vec{T}=J\left[\begin{array}{cc}
\omega^{2}+\frac{1}{2} \hat{\Omega}^{2}+\frac{1}{2} \hat{\Omega}^{2} \cos (2 v t) & -j 2 \omega \hat{\Omega} \cos (v t)+j v \hat{\Omega} \sin (v t) \\
j 2 \omega \hat{\Omega} \cos (v t)-j v \hat{\Omega} \sin (v t) & \omega^{2}+\frac{1}{2} \hat{\Omega}^{2}+\frac{1}{2} \hat{\Omega}^{2} \cos (2 v t)
\end{array}\right]\left[\begin{array}{c}
\hat{\vartheta} \\
\hat{\varphi}
\end{array}\right] .
$$

The torque $\vec{T}$ acts on the haltere, which is part of the isotropic gyroscopic mechanical system. The response of this system is described by a coupled second order differential equation: 


$$
\left[\begin{array}{cc}
G(\omega, v) & -C(\omega, v) \\
-C(\omega, v) & G(\omega, v)
\end{array}\right]\left[\begin{array}{c}
\hat{\vartheta} \\
\hat{\varphi}
\end{array}\right]=\left[\begin{array}{c}
\mathcal{T}_{\text {ext }} \\
0
\end{array}\right]
$$

where $G(\omega, v)$ is given by:

$$
G(\omega, v)=-\omega^{2}+j 2 \zeta \omega \omega_{0}+\omega_{0}^{2}-\frac{1}{2} \hat{\Omega}^{2}[1+\cos (2 v t)]
$$

and $C(\omega, v)$ denotes the coupling between the drive and sense mode due to angular rates:

$$
C(\omega, v)=j 2 \omega \hat{\Omega} \cos (v t)-j v \hat{\Omega} \sin (v t) .
$$

In these expressions, the resonance frequency $\omega_{0}$, damping ratio $\zeta$ and normalized driving torque $\mathcal{T}_{\text {ext }}$ are defined as

$$
\omega_{0}=\sqrt{\frac{S}{J}}, \quad \zeta=\frac{R}{2 \sqrt{S J}}, \quad \mathcal{T}_{\text {ext }}=\frac{T_{0}}{J} .
$$

where $T_{0}$ is the driving torque ${ }^{2}, S$ is the torsional stiffness, $R$ is the torsional resistance and $J$ is the moment of inertia. Further, $\hat{\vartheta}$ and $\hat{\varphi}$ are the amplitudes of respectively drive and sense mode. For constant angular rates $(v=0)$, the second order mechanical system reduces to:

$$
\left[\begin{array}{cc}
-\omega^{2}+j 2 \zeta \omega \omega_{0}+\omega_{0}^{2}-\hat{\Omega}^{2} & -j 2 \omega \hat{\Omega} \\
j 2 \omega \hat{\Omega} & -\omega^{2}+j 2 \zeta \omega \omega_{0}+\omega_{0}^{2}-\hat{\Omega}^{2}
\end{array}\right]\left[\begin{array}{c}
\vartheta \\
\varphi
\end{array}\right]=\left[\begin{array}{c}
\mathcal{T}_{\text {ext }} \\
0
\end{array}\right]
$$

From (8.6) and (8.10) follows that the exposure to an angular rate $\Omega$ clearly affects the system's behaviour; its resonance frequency is reduced by centrifugal forces, and torque contributions by angular Coriolis forces cause coupling between drive and sense mode. Although the reduction in resonance frequency by $\hat{\Omega}^{2}$ is typically negligible, its principle can be used for gyroscopic measurement of large angular rates (Zotov et al., 2011).

\subsubsection{Design rules}

The governing equation of motion for the drive mode with amplitude has already been stated in (8.6). By driving the gyroscope by a harmonic torque, assuming

\footnotetext{
${ }^{2}$ This torque is delivered by electrostatic actuation in the case of the MEMS gyroscope and by the muscles in case of the fly.
} 
that the angular rate $\Omega$ is small compared to the sensor's resonance frequency $\left(\hat{\Omega} \ll \omega_{0}\right)$, and angular accelerations are small $(v \ll \omega)$, the drive mode amplitude $\hat{\vartheta}$ can be approximated well by:

$$
\hat{\vartheta}(\omega)=\frac{\mathcal{T}_{\text {ext }}}{\sqrt{\left(\omega_{0}^{2}-\omega^{2}\right)^{2}+\left(2 \zeta \omega_{0} \omega\right)^{2}}} .
$$

Generally, MEMS gyroscopes are driven at a frequency $\omega$ equal to the gyroscope's resonance frequency $\omega_{0}$ in the case of underdamped systems. Then, the drive mode amplitude can be simplified to:

$$
\hat{\vartheta}=\frac{\mathcal{T}_{\text {ext }}}{2 \zeta \omega_{0}^{2}} .
$$

Based on the drive mode motion, the normalized Coriolis-induced torque $\mathcal{T}_{\mathrm{c}}$ amplitude of the sense mode is given by:

$$
\mathcal{T}_{\mathrm{c}}=2 \omega_{0} \hat{\vartheta} \hat{\Omega} .
$$

Using the system of equations of (8.6), the corresponding sense mode amplitude $\hat{\varphi}$ can be approximated as:

$$
\hat{\varphi}=\frac{\mathcal{T}_{\text {ext }}}{2} \frac{1}{\omega_{0}^{3} \zeta^{2}} \hat{\Omega} .
$$

Now, we define the ratio $\mathcal{R}$ between sense and drive mode amplitudes, which is a measure for the system's responsivity ${ }^{3}$. Since Coriolis forces in general are small, a large $\mathcal{R}$ is desirable in order to measure correspondingly small angular rates $\Omega$. The ratio $\mathcal{R}$ is defined as:

$$
\mathcal{R}=\frac{\mathrm{d}}{\mathrm{d} \hat{\Omega}}\left(\frac{\hat{\varphi}}{\hat{\vartheta}}\right)=\kappa .
$$

where $\kappa$ is a design parameter, which is the reciprocal of the product of damping ratio $\zeta$ and resonance frequency $\omega_{0}$ :

$$
\kappa=\frac{1}{\zeta \omega_{0}} .
$$

However, in case the gyroscope is critically damped or overdamped, the gyroscope should be operated below its resonance frequency $\omega_{0}$. A suitable frequency of operation is when the damping term of (8.9) becomes equal to the stiffness-related

\footnotetext{
${ }^{3}$ This ratio is related to the scale factor of MEMS gyroscopes by taking the transfer by capacitive read-out into account (IEEE Aerosp. Electron. Sys. Soc., 2001).
} 
term for small angular rates $\Omega$, and the actuation frequency is chosen half this frequency:

$$
2 \zeta \omega_{0} \omega=\omega_{0}^{2}, \quad \text { giving } \quad \omega=\frac{\omega_{0}}{2 \zeta} .
$$

By operating the device in such a way, in both the drive and sense mode the factor $2 \zeta$ drops, but the expression for the Coriolis torque given by (8.13) remains unchanged. Therefore, by this analysis, the ratio of the drive and sense mode amplitudes of a gyroscope is equal for all classes of damping.

The performance of a gyroscope is also to be determined by its response time; besides a sensitive sensory system, also the ability to quickly respond to changes in angular rate $\Omega$ classifies the system. The corresponding response time $\tau_{95}$ - a measure for agility — is calculated from the second order differential equation, describing the mechanical behaviour of the system in response to a torque step function. Here, we assume that the gyroscope is driven in steady state with frequency $\omega$, the coupling from sense to drive mode is negligible, and an angular rate $\Omega$ is applied stepwise $(v=0)$. In that case, the governing equation for analyzing transients in the sense mode becomes

$$
\frac{\mathrm{d}^{2} \varphi(t)}{\mathrm{d} t^{2}}+2 \zeta \omega_{0} \frac{\mathrm{d} \varphi(t)}{\mathrm{d} t}+\left(\omega_{0}^{2}-\hat{\Omega}^{2}\right) \varphi(t)=2 \omega \hat{\Omega} \hat{\vartheta} \sin (\omega t) H(t),
$$

where $H(t)$ denotes the Heaviside step function. The general solution of this damped second order system is:

$$
\varphi(t)=\Phi_{1} e^{-r_{1} t}+\Phi_{2} e^{-r_{2} t},
$$

where $\Phi_{1}$ and $\Phi_{2}$ need to be derived from initial conditions and $r_{1}$ and $r_{2}$ are given by:

$$
r_{1,2}=\zeta \omega_{0} \pm \sqrt{\zeta^{2} \omega_{0}^{2}-\omega_{0}^{2}+\hat{\Omega}^{2}}
$$

which simplifies under the condition $\hat{\Omega} \ll \omega_{0}$ to

$$
r_{1,2} \approx \omega_{0}\left(\zeta \pm \sqrt{\zeta^{2}-1}\right)
$$

The general solution (8.19) is either real or complex. When the damping ratio $\zeta$ is equal or greater than one, the solution is real and the hair moves without oscillations towards the point of torque equilibrium, dominated by the slow moving mathematical term. When the damping ratio $\zeta$ becomes smaller than one, the square root term becomes imaginary and the hair shows overshoot towards 
its equilibrium, leading to a longer stabilization time. The response time $\tau_{95}$, indicated as the time to get stable within $95 \%$ of the end value, is approximated ${ }^{4}$ as:

$$
\tau_{95} \approx \begin{cases}\frac{3}{\zeta \omega_{0}} & \text { if } \zeta<0.5, \\ \frac{6 \zeta}{\omega_{0}} & \text { if } \zeta>1 .\end{cases}
$$

The best response time is obtained when $\zeta=0.707$, corresponding to a close to critically damped system (Shinners, 1998). However, we observe that nearly all described gyroscopic systems shown in figure 8.3 are clearly underdamped.

Another consequence of the damping ratio is the impact on the measurement bandwidth $\Delta \omega$ for a gyroscope. From (8.6) we observe that on applying an angular rate $\Omega$ with frequency $v$, a response is induced in the sense mode at frequencies $\omega \pm v$, which can be calculated analytically by using e.g. the method of harmonic balancing as described in (Cartmell, 1990). To measure the response, the gyroscope needs sufficient bandwidth $\Delta \omega$ :

$$
\Delta \omega \geq v .
$$

Based on the definition of the quality factor $Q$, the bandwidth $\Delta \omega$ for underdamped systems is found from the Full Width at Half Maximum (FWHM):

$$
Q=\frac{\omega_{0}}{2 \Delta \omega}, \quad \text { giving } \quad \Delta \omega=\frac{1}{\mathcal{K}},
$$

for which $\Delta \omega$ depends inversely on the design parameter $\kappa$ and we still assume that the angular rate $\Omega$ is small compared to the sensor's resonance frequency $\left(\hat{\Omega} \ll \omega_{0}\right)$, and angular accelerations are small $(v \ll \omega)$. For overdamped systems, we assume that the system is driven below resonance and the bandwidth is equal to the frequency of operation, defined in (8.17). Consequently, the bandwidth $\Delta \omega$ becomes:

$$
\Delta \omega \approx \begin{cases}\zeta \omega_{0} & \text { if } \zeta<0.5, \\ \frac{\omega_{0}}{4 \zeta} & \text { if } \zeta>1 .\end{cases}
$$

Similar to the response time $\tau_{95}$, a spline interpolation technique can be used to obtain $\Delta \omega$ for all values of $\zeta$.

\footnotetext{
${ }^{4} \mathrm{~A}$ precise mathematical solution is rather involved. A spline interpolation is used for connecting the two asymptotes smoothly and obtaining a good approximation, which is not shown here.
} 
The sense/drive ratio, the response time and measurement bandwidth are all taken as a criterion for designing a gyroscope. However, these quantities exhibit a similar proportionality with respect to the design parameter $\kappa$ for underdamped gyroscopes:

$$
\tau_{95} \propto \kappa, \quad \quad \mathcal{R} \propto \kappa, \quad \Delta \omega \propto \frac{1}{\kappa} .
$$

As a consequence, a trade-off exists between the sensitivity parameter $\mathcal{R}$ and the response time $\tau_{95}$ and bandwidth $\Delta \omega$ (see also (Apostolyuk et al., 2002)). Namely, to have a good sensitivity $\mathcal{R}$, a large $\mathcal{K}$-value is required, but to obtain a fast and wide-band responding system, $\mathcal{k}$ should be small. For design and analysis, we capture this trade-off in a single number by defining a figure of merit:

Figure of merit $=$ Sense $/$ drive ratio $\times$ Bandwidth $=\mathcal{R} \times \Delta \omega$.

An overview of several MEMS gyroscopic systems and the blowfly's haltere-based gyroscope (Nalbach, 1993) is shown in figure 8.3 by evaluating the figure of merit as function of the damping ratio $\zeta$. We observe that most MEMS gyroscopes are clearly designed with focus on achieving high responsivity at the cost of bandwidth. Especially the gyroscopes described in (Trusov et al., 2011; Prikhodko et al., 2011) show very low damping factors while having a resonance frequency at $2 \mathrm{kHz}$, being highly sensitive to angular rate, since the ultimate purpose of these two gyroscopes is angle measurement rather than angular rate measurement. Contrarily, the fly's haltere is clearly geared towards a large measurement bandwidth and a fast response. Also, MEMS-based gyroscopes typically have operating frequencies which are $1 \mathrm{kHz}$ or more, which is significantly higher than the $150 \mathrm{~Hz}$ of the blowfly (Nalbach, 1993). Notice that the figure of merit exhibits a clear decrease when having an overdamped system $(\zeta>1)$, implying that vibratory gyroscopes should preferably be underdamped $(\zeta<1)$.

\subsubsection{Thermal noise}

An important characteristic of a sensory system is its Signal-to-Noise Ratio (SNR). Generally, when the SNR is equal or higher than one, a system may allow to detect the input signal. Hence, besides the responsivity of the sensor, the noise level of the system determines the fundamental detection threshold of the system. To find the detection threshold of our system, we assume a power spectral density given by Johnson-Nyquist (thermal) white noise (Gabrielson, 1993):

$$
\overline{T_{\mathrm{n}}^{2}}=4 k_{\mathrm{B}} T_{0} R,
$$




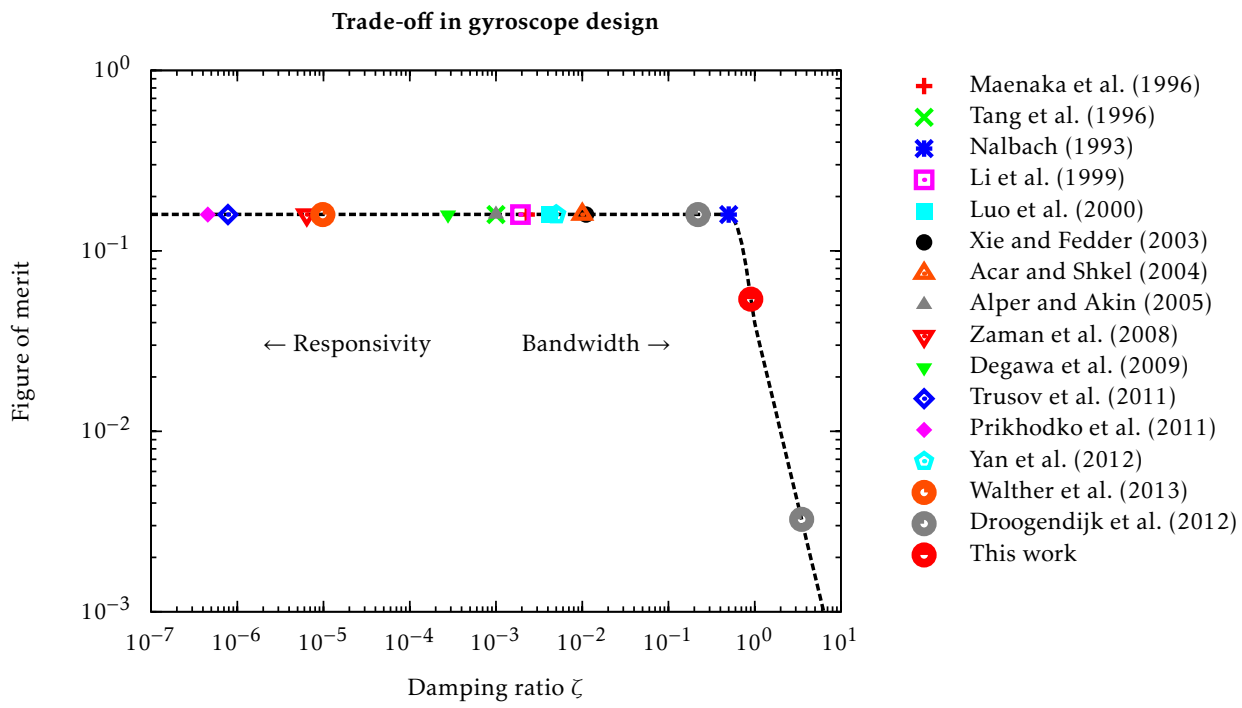

Figure 8.3: Figure of merit for designing a gyroscopic system. The dashed line indicates the figure of merit by (8.27).

where $k_{\mathrm{B}}$ is the Boltzmann's constant, $T_{0}$ is the ambient temperature and $\bar{T}_{n}^{2}$ the square of the noise induced equivalent torque per unit of bandwidth. The mechanical transfer function $G(\omega)$ of the system determines the resulting mechanical response to the noise:

$$
G(\omega)=\frac{1}{S-J \omega^{2}+j R \omega} .
$$

The equivalent noise angle $\Phi_{\mathrm{n}}$ is found by integrating the noise power over the full spectrum and taking the square root:

$$
\Phi_{\mathrm{n}}=\sqrt{\int_{0}^{\infty} \overline{T_{\mathrm{n}}^{2}}|H(\omega)|^{2} \mathrm{~d} \omega} .
$$

The detection threshold $\Omega_{\mathrm{th}}$ is found by dividing the sensor's equivalent noise angle $\Phi_{\mathrm{n}}$ by the rotational angle $\Phi_{\mathrm{m}}$ for an angular rate of $1^{\circ} \mathrm{s}^{-1}$ :

$$
\Omega_{\mathrm{th}}=\frac{\Phi_{\mathrm{n}}}{\left.\Phi_{\mathrm{m}}\right|_{\hat{\Omega}=1{ }^{\circ} \mathrm{s}^{-1}}} .
$$

Thermal noise calculations for our hair mechanical system indicate a detection 


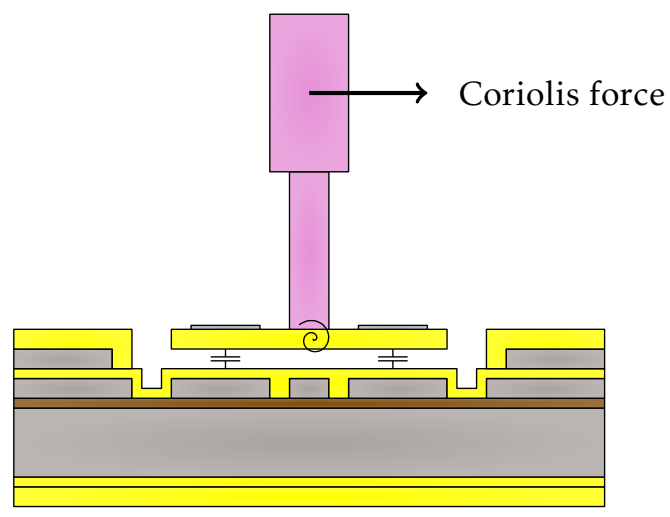

Figure 8.4: Schematic view of the biomimetic gyroscope (side view).

threshold for angular rate sensing of about $0.038^{\circ} \mathrm{s}^{-1}$ for frequencies within a bandwidth of $1 \mathrm{kHz}$.

\subsubsection{Design}

The design of the biomimetic gyroscope is based upon the fabrication process for biomimetic hair flow sensors described by Bruinink et al. (2009) and Dagamseh et al. (2011) to realize a haltere-like structure. Our gyroscope will be actuated in the drive mode with angle $\vartheta$ (see figure 8.2) by exploiting electrostatic actuation. Electrostatic forces induced by judiciously chosen ac-voltages bring the artificial haltere in a steady-state resonant rotational motion. Measurement of the Coriolisinduced movement of the artificial haltere in the sense direction with rotational angle $\varphi$ is achieved by measuring the differential change in capacitance upon tilt of the artificial haltere (figure 8.4).

Although we reported on the design and fabrication of such a gyroscope earlier (Droogendijk et al., 2012), we implemented some important design adjustments here. The suspension of the haltere-based gyroscope is changed to a gimbalsuspension, like the gyroscope described by Acar and Shkel (2004). Doing so, the effects of residual stress by having a bi-layer suspension (i.e. SiRN and $\mathrm{Al}$ ) can be significantly reduced. Also, the drive and sense modes get mechanically distinguishable, allowing for a separate optimisation of both modes. A schematic view of the two modes for our gimbal suspension is given in figure 8.5. A disadvantage of such a gimbal-design is the difficulty to achieve mode-matching (between drive and sense), which is often aimed for in MEMS gyroscopes to achieve better performance (see e.g. (Hu et al., 2011; Sonmezoglu et al., 2012)). However, for the biomimetic approach followed in this work, the quality factor $Q$ of a gyroscope is 


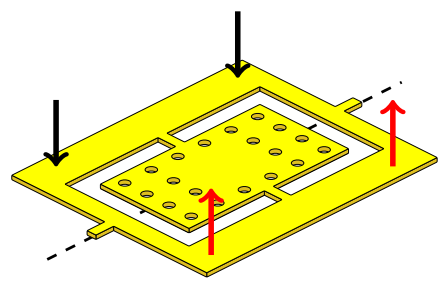

(a) Drive mode.

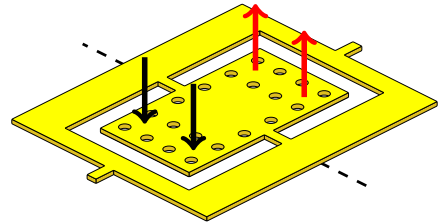

(b) Sense mode.

Figure 8.5: Characteristic modes of the gimbal gyroscope.

relatively low, and consequently mode-matching plays a less pivotal role for the gyroscope's performance.

\subsection{Fabrication}

An overview of the design and the fabrication process for the biomimetic gyroscope is shown in figure 8.6. The sensor is fabricated on a Silicon-On-Insulator wafer. Trenches are etched in the silicon device layer using DRIE. A layer of $200 \mathrm{~nm}$ stoichiometric $\mathrm{Si}_{3} \mathrm{~N}_{4}$ is used for covering and protecting the trenches. The device layer contains two electrodes, which are used for capacitive readout of the acceleration-induced movement. On top of the $\mathrm{Si}_{3} \mathrm{~N}_{4}$ layer, a sacrificial layer of poly-silicon $(1.5 \mu \mathrm{m})$ is deposited by LPCVD. The sensor membrane and springs are constructed by depositing and patterning a $1 \mu \mathrm{m}$ SiRN layer on top of the poly-silicon. Aluminium $(80 \mathrm{~nm})$ is sputtered on top of the membrane to create the electrodes for capacitive read-out. Our artificial clavate hair is created by two layers of SU-8, to realize both the centre of mass towards the top of the hair structure and a total hair length of about $800 \mu \mathrm{m}$ and an average diameter of about $80 \mu \mathrm{m}$. Finally, to release the membrane the sacrificial poly-silicon layer is removed using $\mathrm{XeF}_{2}$ etching. The fabrication results are shown by the SEM image in figure 8.7 .

\subsection{Experimental}

\subsubsection{Setup}

To characterize the mechanical properties of the gyroscope, an experimental setup based on Laser Doppler Vibrometry (Polytec, 2005) is used (figure 8.8). To measure the rotational modes we differentially applied a sinusoidal signal 


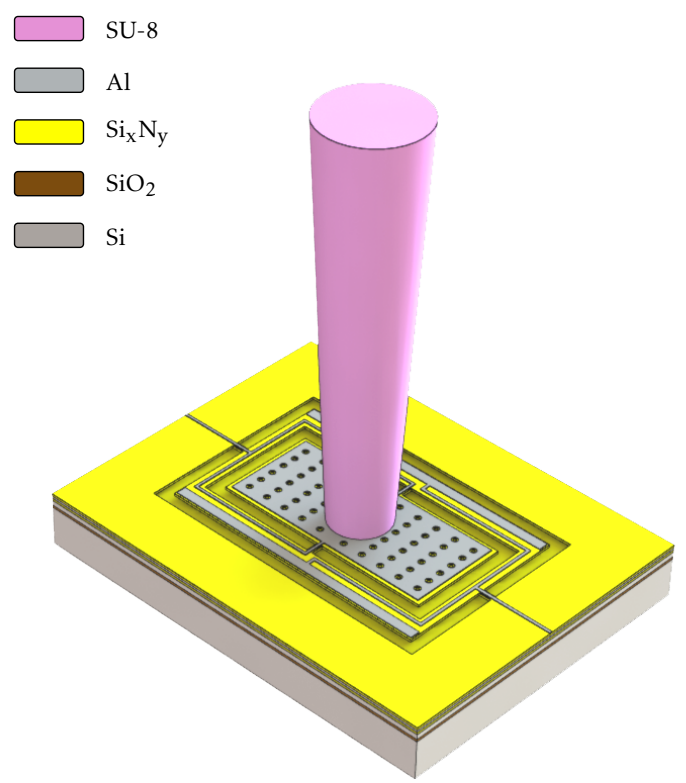

Figure 8.6: Schematic view of the MEMS hair-based gyroscope.

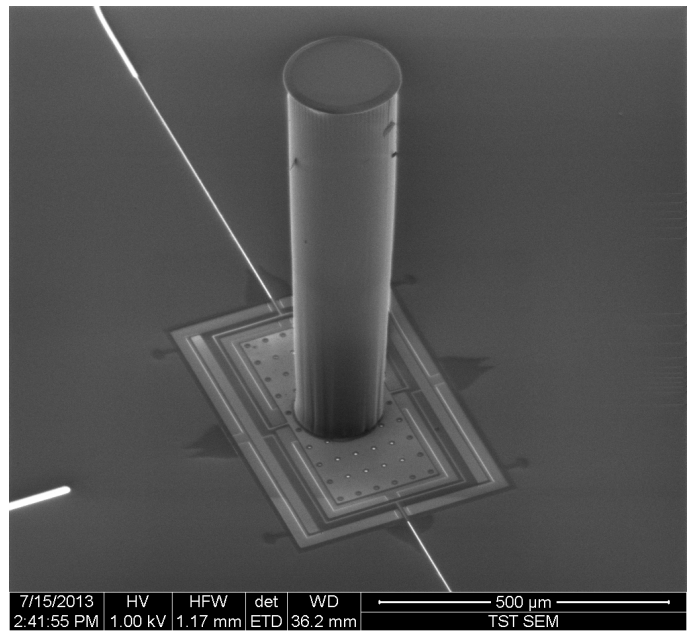

Figure 8.7: MEMS hair-based gyroscope fabricated by surface micromachining and using SU-8 lithography.

superposed to a dc-offset voltage (Delta Elektronika - Power Supply E 030-1) to a pair of electrodes. 


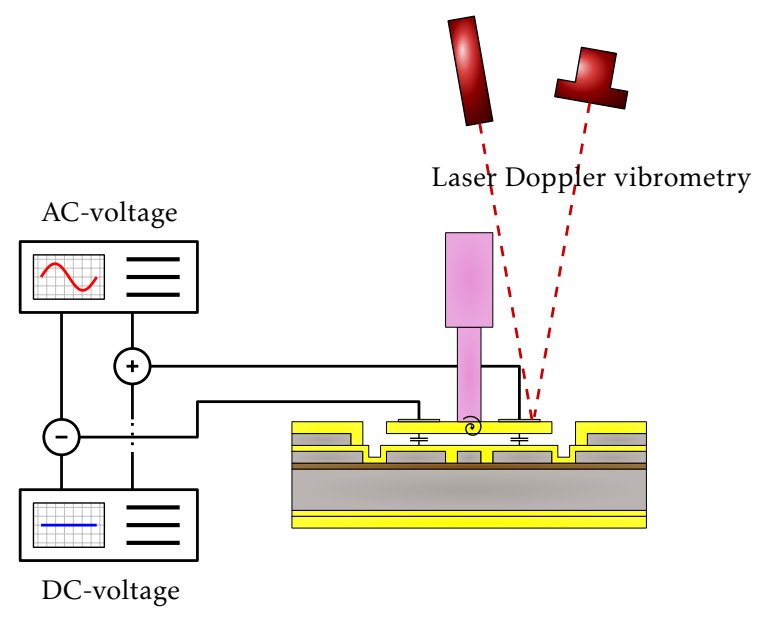

Figure 8.8: Experimental setup for characterization of the biomimetic gyroscope.

\subsubsection{Frequency response}

The response for the drive mode (figure 8.5a) was measured by applying a chirpvoltage in push-pull configuration. The magnitude and phase response are shown in figure 8.9, both together with the results of an analytical model based on a classical second-order mechanical system. From both figures, the drive mode turns out to be slightly overdamped $(\zeta=0.9)$ with its resonance frequency found at approximately $550 \mathrm{~Hz}$. Due to the overdamped nature of the system, the bandwidth of the drive mode is calculated by computing the intersection of the stiffness and damping contributing terms in (8.6) and is found to be $305 \mathrm{~Hz}$. Further, we observe that there is a good agreement between the analytical model and the measured response for frequencies up to about $800 \mathrm{~Hz}$.

Similar measurements have been made for the sense mode (figure 8.5b). From figure 8.10, the sense mode turns out to be slightly overdamped $(\zeta=1.1)$ with its resonance frequency found at approximately $300 \mathrm{~Hz}$. Notice that the peaks around this frequency are not due to mechanical resonance, but are caused by limitations within the used laser Doppler vibrometry setup. Due to the overdamping, the bandwidth of the sense mode is calculated by computing the intersection of the stiffness and damping contributing terms in (8.6) and is found to be $137 \mathrm{~Hz}$. Further, we observe that there is a good agreement between the analytical model and the measured response for frequencies up to about $2 \mathrm{kHz}$. 

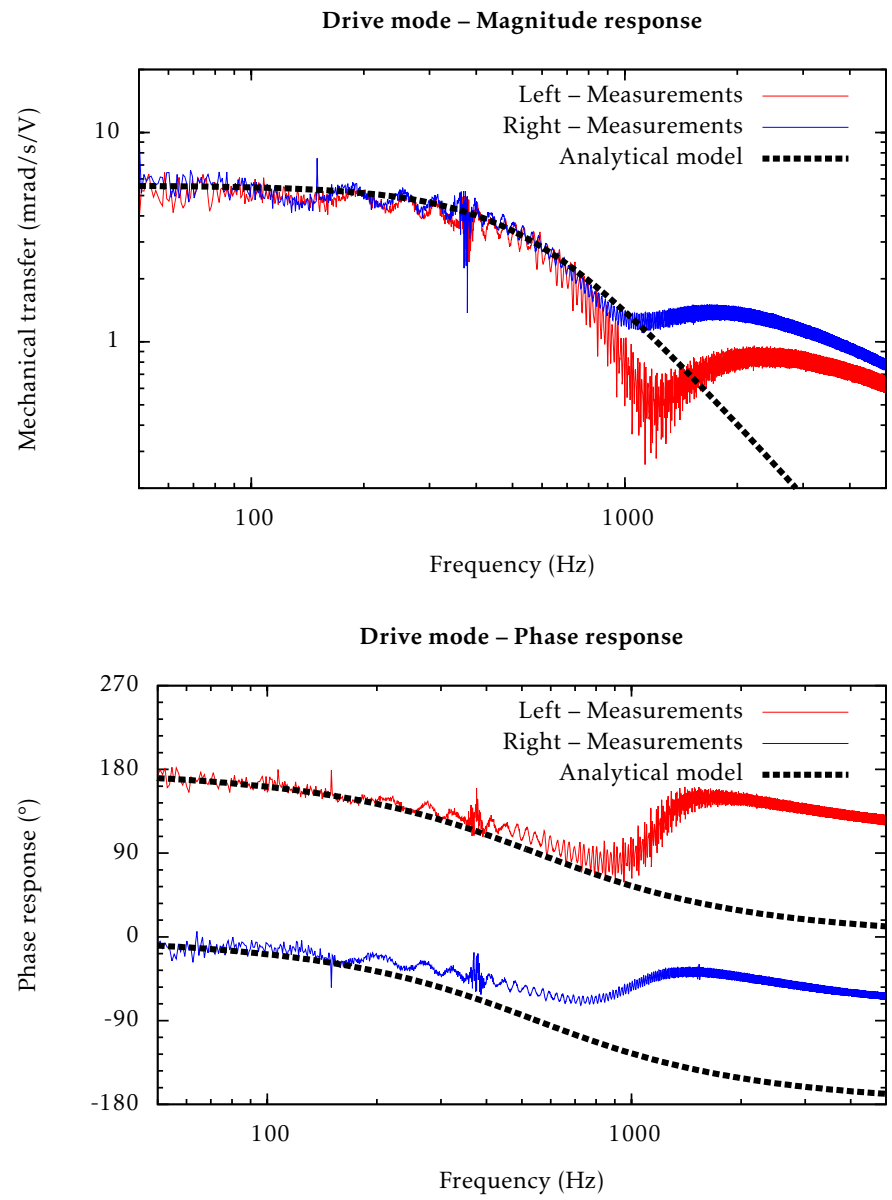

Figure 8.9: Optically measured response of the membrane displacement by electrostatically actuating the drive mode $\left(f_{0}=550 \mathrm{~Hz}\right.$ and $\left.\zeta=0.9\right)$.

\subsection{Discussion}

\subsubsection{Fabrication}

Regarding the fabrication process, the SEM image from the biomimetic gyroscope (figure 8.7) shows that we have successfully fabricated artificial haltere-structures with increased mass at the top. Optical measurements indicate that the haltere length is about $800 \mu \mathrm{m}$, and that the halteres have a slight negative tapering towards the membrane. This tapering results from the light intensity profile during exposure of the SU-8 as well as the two step lithography process incorporated in 

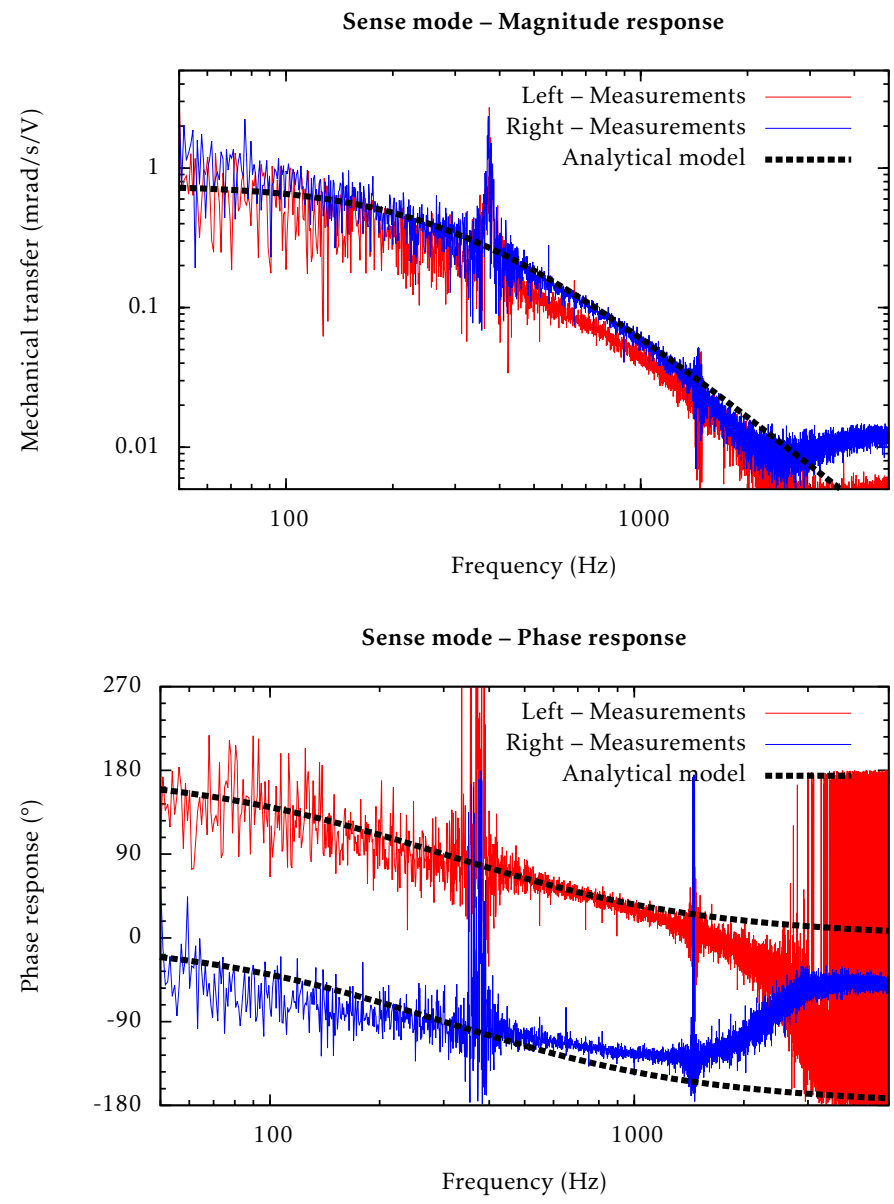

Figure 8.10: Optically measured response of the membrane displacement by electrostatically actuating the sense mode $\left(f_{0}=300 \mathrm{~Hz}\right.$ and $\left.\zeta=1.1\right)$.

the design. This effectively causes the centre of mass to be located in the upper part of the halteres, similar to the fly's haltere (figure 8.1).

\subsubsection{Modal response}

The measured resonance frequency for the drive mode was measured to be about $510 \mathrm{~Hz}$ with a damping ratio of $\zeta=0.9$. Although this resonance frequency is slightly higher than the wing-beat frequency of the fly $(130-150 \mathrm{~Hz})$, it remains in the same order of magnitude. Similar results are obtained for the sense mode, wherein the resonance frequency was found to be about $300 \mathrm{~Hz}$ with a slightly 
overdamped system $(\zeta=1.1)$.

However, we observed that for both measurements the response is a combination of modes, especially for higher excitation frequencies. Since every mode is expected to be about critically damped, it is difficult to identify each modal resonance frequency due to the absence of clear resonance peaks and phase shifts. Theoretically, using capacitive differential read-out techniques, the contributions of the drive and vertical mode can be commonly rejected for measurement of the sense mode.

\subsubsection{Biomimetic approach}

A haltere-inspired gyroscope has been designed, fabricated and partially characterized. However, we were not able to experimentally demonstrate its response to externally applied angular rates using our home-built rate-table, electrostatic actuation of the drive mode, and capacitive read-out of the sense mode. With the current design and the maximum applicable angular rate using our rate table (rate amplitude of about $3000^{\circ} \mathrm{s}^{-1}$ at a frequency of $14 \mathrm{~Hz}$ ), the calculated displacement of the membrane edges for capacitive read-out of the sense mode is in the order of $\mathrm{nm}$, which corresponds to the measurement threshold of our read-out electronics (Dagamseh et al., 2013).

The high damping ratio $\zeta$ for this type of gyroscope results in two difficulties for rate measurements. First, following (8.26) from our design analysis a trade-off exists between responsivity (ratio drive/sense mode), and bandwidth and response time. Clearly, a high damping ratio has a negative impact on the responsivity, thus requiring a relative large drive angle amplitude for a given sense angle amplitude. In addition, when having a critically or overdamped electrostatically driven system, the driving can be considered quasi-statically (i.e. operating well below resonance). For a torsional electrostatic actuator, the maximum achievable drive angle $\vartheta$, while preventing pull-in, is (Nielson and Barbastathis, 2006):

$$
\vartheta=0.4404 \vartheta_{0}, \quad \text { with } \quad \vartheta_{0}=\arctan \left(\frac{g_{\mathrm{m}}}{L_{\text {drive }}}\right) \text {, }
$$

where $g_{\mathrm{m}}$ is the gap between the gimbal and the substrate, $L_{\text {drive }}$ is half the length of the gimbal in the drive direction and $\vartheta_{0}$ is the maximum mechanical angle at which the gimbal hits the lower substrate. To compare, when the system is underdamped and electrostatically driven at resonance, the gimbal's rotation is amplified by the Q-factor, resulting in a larger sense amplitude, but smaller bandwidth and increased response time.

Generally, we believe that a working biomimetic gyroscope following the 
'design rules' of a fly and the use of MEMS technology is difficult to achieve, but possible. However, this will require adaptation of the design and the fabrication process. For our gyroscope, in particular the value of $\mathcal{R}$ needs to be increased in order to measure the applied angular rates. A possibility is the use of piezoresistive sensing instead of capacitive read-out, as proposed by Wicaksono et al. (2007). Also, improvement of the mechanical gimbal-suspension and the use of more compliant materials will help to increase $\mathcal{R}$. One could also think of exchanging the technology of MEMS for another type of technology (e.g. 3Dprinting) and investigate its potential for biomimetic gyroscopes.

\subsubsection{Comparison to the fly's haltere}

To compare the biomimetic gyroscope with the fly's haltere, several performance metrics of the fly's haltere are identified. First, the moment of inertia $J$ is calculated based on the values given by Nalbach (1993), wherein the mass is considered as a point mass at distance $r_{\mathrm{h}}$ from the axis of rotation. Considering the fly's haltere to be slightly underdamped $(\zeta=0.5)$, the resonance frequency $f_{0}$, torsional stiffness and torsional damping can be calculated based on a natural frequency of $150 \mathrm{~Hz}$ (Nalbach and Hengstenberg, 1994). From these parameters, performance metrics as response time and sense/drive ratio are calculated. An overview of all parameters and performance metrics with their values is shown in table 8.1. Also the values for the haltere-inspired gyroscope are listed, wherein most values are theoretical.

From this table, nearly all values for the haltere-inspired gyroscope are in the same order compared to the actual fly's halteres. We observe that the haltere-based gyroscope are geared towards larage bandwidth and short response time rather than achieving a high sense/drive ratio $\mathcal{R}$, which is also exhibited by the figure of merit (figure 8.3). Notice that to achieve sufficient rotation in the sense mode the fly's haltere operates under large drive angles with amplitudes up to $85^{\circ}$, whereas the maximum achievable drive amplitude for the MEMS gyroscope is only $0.5^{\circ}$. As a consequence, the response in sense direction, which is linearly related to the drive amplitude, is significantly larger for the fly's haltere than for our MEMS counterpart. Furthermore, by having these large drive amplitudes, the fly is also able to measure angular rates for all three planes: roll, pitch and yaw (Nalbach, 1993).

The response time for the fly is theoretically calculated to be about $4.5 \mathrm{~ms}$, which is in the order of the fly's latency $(<5 \mathrm{~ms})$ for making compensatory head movements (Hengstenberg et al., 1986). Although thermal noise plays an important role in the flow-sensitive filiform hairs of crickets (Shimozawa et al., 
Table 8.1: Characteristics of the fly's haltere based on (Nalbach, 1993) and the biomimetic gyroscope. Values marked with ${ }^{\star}$ are measured quantities, others are calculated from geometry, materials properties, or are derived from dynamic characterization.

\begin{tabular}{|c|c|c|c|}
\hline Quantity & Symbol & Fly & This work (drive) \\
\hline \multicolumn{4}{|l|}{ Haltere } \\
\hline Length & $r_{\mathrm{h}}$ & $1.07 \mathrm{~mm}$ & $0.8 \mathrm{~mm}$ \\
\hline Mass & $m_{\mathrm{h}}$ & $5.89 \times 10^{-9} \mathrm{~kg}$ & $2.29 \times 10^{-8} \mathrm{~kg}$ \\
\hline \multicolumn{4}{|l|}{ Characteristics } \\
\hline Damping ratio & $\zeta$ & 0.5 & $0.9^{*}$ \\
\hline Resonance frequency & $f_{0}$ & $210 \mathrm{~Hz}$ & $550 \mathrm{~Hz}^{*}$ \\
\hline \multicolumn{4}{|l|}{ Parameters } \\
\hline Torsional stiffness & $S$ & $1.17 \times 10^{-8} \mathrm{Nm} \mathrm{rad}^{-1}$ & $4.89 \times 10^{-8} \mathrm{Nm} \mathrm{rad}^{-1}$ \\
\hline Torsional damping & $R$ & $8.90 \times 10^{-12} \mathrm{Nmsrad}^{-1}$ & $2.79 \times 10^{-11} \mathrm{Nms} \mathrm{rad}^{-1}$ \\
\hline Moment of inertia & $J$ & $6.74 \times 10^{-15} \mathrm{~kg} \mathrm{~m}^{2}$ & $4.93 \times 10^{-15} \mathrm{~kg} \mathrm{~m}^{2}$ \\
\hline \multicolumn{4}{|l|}{ Performance } \\
\hline Response time & $\tau_{95}$ & $4.55 \mathrm{~ms}$ & $\sim 1.2 \mathrm{~ms}$ \\
\hline Sense/drive ratio & $\mathcal{R}$ & $2.65 \times 10^{-5 \circ-1} \mathrm{~s}^{-1}$ & $5.61 \times 10^{-6 \circ-1} \mathrm{~s}^{-1}$ \\
\hline Bandwidth (sense) & $\Delta \omega$ & $105 \mathrm{~Hz}$ & $137 \mathrm{~Hz}$ \\
\hline Detection threshold & $\Omega_{\mathrm{th}}$ & $0.038^{\circ} \mathrm{s}^{-1}$ & $0.018^{\circ} \mathrm{s}^{-1}$ \\
\hline Drive angle & $\vartheta_{\max }$ & $85^{\circ}$ & $0.5^{\circ}$ \\
\hline
\end{tabular}

2003), we do not believe that thermal noise matters for angular rate measurement. Hengstenberg (1991) showed that below angular rates of $50^{\circ} \mathrm{s}^{-1}$ no body motion of the fly was observed in absence of visual clues. In addition, he showed that the fly is able to respond to angular step stimuli of up to $2000^{\circ} \mathrm{s}^{-1}$, indicating that the fly tends to respond to large variations in angular rate $\Omega$ rather than to small angular rates.

\subsection{Conclusions}

Inspired by the fly's haltere, a biomimetic gimbal-based gyroscope has been designed, fabricated and partially characterized. Design rules for gyroscopes are investigated and it was shown that haltere-based gyroscopes tend to emphasize a large measurement bandwidth and a fast response, rather than a high responsivity or low detection threshold, by having a relatively low $\mathrm{Q}$-factor. Measurements on 
our haltere inspired sensors indicate an excitable gyroscope with a (drive mode) resonance frequency of about $550 \mathrm{~Hz}$ and a damping ratio of 0.9. A response to externally applied angular rates is not demonstrated due to the limited applied rotation rates and the very small induced sense mode amplitude, which is mainly caused by the structures being overdamped and the small achievable drive mode amplitudes; our haltere-based gyroscope is physically limited to drive angles of about $0.5^{\circ}$, which is much smaller than the fly's drive angles (up to $85^{\circ}$ ).

\section{References}

Acar, C. and Shkel, A. M. (2004). Structural design and experimental characterization of torsional micromachined gyroscopes with non-resonant drive mode. J. Micromech. Microeng., 14:15-25.

Alper, S. E. and Akin, T. (2005). A single-crystal silicon symmetrical and decoupled MEMS gyroscope on an insulating substrate. J. Microelectromech. S., 14(4):707717.

Apostolyuk, V. A., Logeeswaran, V. J., and Tay, F. E. H. (2002). Efficient design of micromechanical gyroscopes. J. Micromech. Microeng., 12:948-954.

Bruinink, C. M., Jaganatharaja, R. K., de Boer, M. J., Berenschot, J. W., Kolster, M. L., Lammerink, T. S. J., Wiegerink, R. J., and Krijnen, G. J. M. (2009). Advancements in technology and design of biomimetic flow-sensor arrays. In Proc. MEMS 2009, pp. 152-155.

Cartmell, M. (1990). Introduction to linear, parametric and nonlinear vibrations. Chapmann and Hall, London, $1^{\text {st }}$ edition.

Challoner, A. D., Gutierrez, R. C., and Tang, T. K. (2007). Cloverleaf microgyroscope with electrostatic alignment and tuning. Patent. US 7159441.

Chan, W. P., Prete, F., and Dickinson, M. H. (1998). Visual input to the efferent control system of a fly's "gyroscope". Science, 280:289-292.

Dagamseh, A., Lammerink, T., Sanders, R., Wiegerink, R., and Krijnen, G. (2011). Towards high-resolution flow cameras made of artificial hair flow-sensors for flow pattern recognition. In Proc. MEMS 2011, pp. 648-651, Cancun, Mexico.

Dagamseh, A. M. K., Bruinink, C. M., Wiegerink, R. J., Lammerink, T. S. J., Droogendijk, H., and Krijnen, G. J. M. (2013). Interfacing of differentialcapacitive biomimetic hair flow-sensors for optimal sensitivity. J. Micromech. Microeng., 23:035010. 
Degawa, M., Jeong, H. W., Sonobe, H., Takubo, C., Yamanaka, K., and Goto, Y. (2009). Laterally-driven deformation-robust MEMS gyroscopes with three sets of summetrically arranged folded-beam suspensions. In Proc. Transducers'09, pp. 664-667, Denver, CO, USA.

Droogendijk, H., Brookhuis, R. A., de Boer, M. J., Sanders, R. G. P., and Krijnen, G. J. M. (2012). Design and fabrication of a biomimetic gyroscope inspired by the fly's haltere. In Proc. IEEE Sensors 2012, pp. 1400-1403, Taipei, Taiwan.

Fraenkel, G. and Pringle, J. W. S. (1938). Biological sciences: halteres of flies as gyroscopic organs of equilibrium. Nature, 141:919-920.

Gabrielson, T. B. (1993). Mechanical thermal noise in micromachined acoustic and vibration sensors. IEEE Trans. Electron. Devices, 40:903-909.

Hengstenberg, R. (1991). Gaze control in the blowfly Calliphora : a multisensory, two-stage integration process. Neurosciences, 3:19-29.

Hengstenberg, R. (1998). Controlling the fly's gyroscopes. Nature, 392:757-758.

Hengstenberg, R., Sandeman, D. C., and Hengstenberg, B. (1986). Compensatory head roll in the blowfly Calliphora during flight. Proc. R. Soc. Lond. B, 227(1249):455-482.

Horn, E. and Bischof, H.-J. (1983). Gravity reception in crickets: The influence of cereal and antennal afferences on the head position. J. Comp. Physiol. A, 150:93-98.

Hu, Z. X., Gallacher, B., Burdess, J. S., Fell, C. P., and Townsend, K. (2011). Precision mode matching of MEMS gyroscopes by feedback control. In Proc. IEEE Sensors 2011, pp. 16-19, Limerick, Ireland.

IEEE Aerosp. Electron. Sys. Soc. (2001). IEEE standard for inertial sensor terminology. IEEE Std. 528-2001.

Li, X., Bao, M., Yang, H., Shen, S., and Lu, D. (1999). A micromachined piezoresistive angular rate sensor with a composite beam structure. Sens. Act. A: Phys., 72:217-223.

Liu, C. (2007). Micromachined biomimetic artificial haircell sensors. Bioinsp. Biomim., 2:162-169.

Luo, H., Fedder, G. K., and Carley, L. R. (2000). An elastically gimbaled z-axis CMOS-MEMS gyroscope. In Proc. IS3M, pp. 1-6, Hong Kong. 
Maenaka, K., Fujita, T., Konishi, Y., and Maeda, M. (1996). Analysis of a highly sensitive silicon gyroscope with cantilever beam as vibrating mass. Sens. Act. A: Phys., 54:568-573.

Nalbach, G. (1993). The halteres of the blowfly Calliphora - I. Kinematics and dynamics. J. Comp. Physiol. A, 173:293-300.

Nalbach, G. and Hengstenberg, R. (1994). The halteres of the blowfly Calliphora - II. Three-dimensional organization of compensatory reactions to real and simulated rotations. J. Comp. Physiol. A, 175:695-708.

Nielson, G. N. and Barbastathis, G. (2006). Dynamic pull-in of parallel-plate and torsional electrostatic MEMS actuators. J. Microelectromech. S., 15(4):811-821.

Polytec (2005). MSA-400 Micro System Analyzer. Polytec, Waldbronn, Germany.

Prikhodko, I. P., Zotov, S. A., Trusov, A. A., and Shkel, A. M. (2011). Sub-degree-perhour silicon MEMS rate sensor with 1 million Q-factor. In Proc. Transducers'11, pp. 2809-2812, Beijing, China.

Pringle, J. W. S. (1948). The gyroscopic mechanism of the halteres of diptera. Philos. Trans. R. Soc. London B., 233(602):347-384.

Ristroph, L., Bergou, A. J., Ristroph, G., Coumes, K., Berman, G. J., Guckenheimer, J., Wang, Z. J., and Cohen, I. (2010). Discovering the flight autostabilizer of fruit flies by inducing aerial stumbles. PNAS, 107(11):4820-4824.

Sandeman, D. C. and Markl, H. (1980). Head movements in flies (Calliphora) produced by deflexion of the halteres. J. Exp. Biol., 85:43-60.

Shimozawa, T. and Kanou, M. (1984). Varieties of filiform hairs: range fractionation by sensory afferents and cercal interneurons of a cricket. J. Comp. Physiol. A, 155:485-493.

Shimozawa, T., Murakami, J., and Kumagai, T. (2003). Cricket wind receptors: thermal noise for the highest sensitivity known. In Sensors and sensing in biology and engineering, pp. 145-159. Springer.

Shinners, S. M. (1998). Modern control system theory and design. John Wiley \& Sons, Inc., $2^{\text {nd }}$ edition.

Smith, G. L., Bedair, S. S., Schuster, B. E., Nothwang, W. D., Pulskamp, J. S., Meyer, C. D., and Polcawich, R. G. (2012). Biologically-inspired, haltere, angular-rate sensors for micro-autonomous systems. In Proc. SPIE, vol. 8373, p. 83731K. 
Sonmezoglu, S., Alper, S. E., and Akin, T. (2012). An automatically mode-matched MEMS gyroscope with $50 \mathrm{~Hz}$ bandwidth. In Proc. MEMS 2012, pp. 523-526, Paris, France.

Tang, T. K., Gutierrez, R. C., Stell, C. B., Vorperian, V., Arakaki, G. A., Rice, J. T., Li, W. J., Chakraborty, I., Shcheglov, K., and Wilcox, J. Z. (1997). A packaged silicon MEMS vibratory gyroscope for microspacecraft. In Proc. MEMS 1997, pp. 500-505, Nagoya, Japan.

Tang, T. K., Gutierrez, R. C., Wilcox, J. Z., Stell, C., Vorperian, V., Calvet, R., Li, W. J., Chakraborty, I., and Bartman, R. (1996). Silicon micromachined vibratory gyroscope. In Proc. Solid-State Sensor and Actuator Workshop, pp. 288-293, Hilton Head Island, SC, USA.

Tobias, M. and Murphey, R. K. (1979). The response of cercal receptors and identified interneurons in the cricket (Acheta domesticus) to airstreams. J. Comp. Physiol. A, 129:51-59.

Trusov, A. A., Prikhodko, I. P., Zotov, S. A., and Shkel, A. M. (2011). Lowdissipation silicon tuning fork gyroscopes for rate and whole angle measurements. IEEE Sens. J., 11(11):2763-2770.

Walther, A., Desloges, B., Lejuste, C., Coster, B., Audebert, P., and Willemin, J. (2013). Development of a 3D capacitive gyroscope with reduced parasitic capacitance. J. Micromech. Microeng., 23:025013.

Wicaksono, D. H. B., Chen, Y., and French, P. J. (2007). Design and modelling of a bio-inspired MEMS gyroscope. In Proc. ICEEI, pp. A-05, Bandung, Indonesia.

Wu, W.-C. and Wood, R. (2006). Angular rate sensor using electromechanical haltere. Patent. US 7107842.

Wu, W.-C., Wood, R. J., and Fearing, R. S. (2002). Halteres for the micromechanical flying insect. In Proc. ICRA 2002, pp. 60-65, Washington, DC, United States.

Xie, H. and Fedder, G. K. (2003). Fabrication, characterization, and analysis of a DRIE CMOS-MEMS gyroscope. IEEE Sens. J., 3(5):622-631.

Yan, L., Shangchun, F., Zhanshe, G., Jing, L., and Le, C. (2012). Study of dynamic characteristics of resonators for MEMS resonant vibratory gyroscopes. Microsyst. Technol., 18:639-647.

Yazdi, N., Ayazi, F., and Najafi, K. (1998). Micromachined inertial sensors. Proc. IEEE, 86(8):1640-1659. 
Zaman, M. F., Sharma, A., Hao, Z., and Ayazi, F. (2008). A mode-matched silicon-yaw tuning-fork gyroscope with subdegree-per-hour allan deviation bias instability. J. Microelectromech. S., 17(6):1526-1536.

Zotov, S. A., Trusov, A. A., and Shkel, A. M. (2011). Demonstration of a wide dynamic range angular rate sensor based on frequency modulation. In Proc. IEEE Sensors 2011, pp. 149-152, Limerick, Ireland. 


\section{9 \\ Bio-inspired angular accelerometer}

\subsection{Introduction}

In biology, the vestibular system is used to detect the head motion in space and results in stabilization of the visual axis, head and body posture (Fernández and Goldberg, 1971). Furthermore, the vestibular system helps with the sense for motion and change in orientation in space. The system consists of two parts: the two otolith organs (the saccule and utricle), which sense linear acceleration (gravity and translational movements), and the three semicircular canals (figure 9.1), which sense angular acceleration in three planes (pitch, roll, and yaw) (Fernández and Goldberg, 1971; Yakushin et al., 1998).

Each semicircular channel is filled with a fluid, where the ends of the channel are connected to a compartment with a sac, called ampulla, containing hair cells. These hair cells are composed of many cilia, which are embedded in a structure called the cupula. As the head rotates the channel moves, but the fluid within the channel lags behind due to its inertia. Consequently, the cupula is deflected and the cilia within as well. As a result, the bending of these cilia alters an electric signal that is transmitted to the brain and forms a measure for the angular acceleration.

This chapter is based on "An angular acceleration sensor inspired by the vestibular system with a fully circular fluid-channel and thermal read-out" by J. Groenesteijn, H. Droogendijk, M. J. de Boer, R. G. P. Sanders, R. J. Wiegerink, and G. J. M. Krijnen, published in Proc. MEMS 2014, pp. 696-699, San Francisco, CA, United States, (2014). 


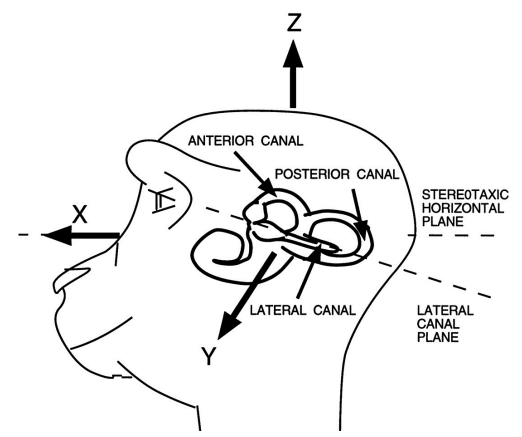

Figure 9.1: Position of the left labyrinth of a monkey, illustrating the three semicircular channels (image taken from (Yakushin et al., 1998)).

Inspired by this vestibular system and the semicircular canals, a MEMS angular accelerometer has been proposed earlier by Arms and Townsend (2003), in which they have a filled semicircular channel with a pressure transducer located at the ends of the channel. Over the past years, also MEMS angular accelerometers have been developed based on other operation principles. Nasiri et al. (2011) realized a MEMS angular accelerometer based on a conventional mass-spring system for measurements in three directions. A different approach is made by Li et al. (2013), in which they propose to use a pendulum-based accelerometer.

Here, we describe a bio-inspired MEMS angular accelerometer, in which the channel is designed to be fully circular and thermal transduction principles are used for measuring the external angular acceleration. That is, by thermally measuring the flow velocity of the channel fluid, its subjection to angular accelerations can be determined. The concept of thermal read-out and a fully circular channel has been described earlier by Ploechinger (2004), but has never been applied using MEMS technology.

\subsection{Theory and modelling}

\subsubsection{Fluid dynamics}

To describe the fluid dynamics inside the circular channel system, we start with the Navier-Stokes equations for incompressible flow (Munson et al., 2006):

$$
\rho\left(\frac{\partial \vec{v}}{\partial t}+\vec{v} \cdot \nabla \vec{v}\right)=-\nabla p+\mu \nabla^{2} \vec{v}+\vec{f},
$$

where the terms on the left-hand side comprise inertial terms and where the 
terms on the right-hand side are depending on pressure, viscosity and body forces represented by $\vec{f}$. Since the fluid flow is inside a cylindrical channel, we will use cylindrical coordinates to describe the fluid dynamics. We assume that there are no pressure gradients in the circular channel $(\nabla p=0)$ and the flow-velocity is only non-zero in the axial direction $z$ with component $u$ and the tube radius is much smaller than the radius of the vestibular circular system $R_{\mathrm{c}}$. A harmonic angular acceleration will lead to a body force density $f$ in the axial direction:

$$
f_{\mathrm{z}}=\rho R_{\mathrm{c}} \alpha_{\mathrm{ext}} \mathrm{e}^{i \omega t},
$$

where $\alpha_{\text {ext }}$ is the amplitude of a harmonic angular acceleration with frequency $\omega$. The Navier-Stokes equations in (9.1) can be simplified to

$$
\mu\left[\frac{\partial^{2} v}{\partial r^{2}}+\frac{1}{r} \frac{\partial v}{\partial r}\right]+f_{z}=0
$$

for Reynold's numbers within the fully laminar flow regime (Peng et al., 1994):

$$
\operatorname{Re}=\frac{\rho V_{0} d}{\mu}<200 .
$$

To find a solution for the fluid velocity-profile $v(r, t)$, we assume a harmonic flow with a parabolic profile (Damiano and Rabbitt, 1996; Vega et al., 2008):

$$
v(r, t)=V_{0} \mathrm{e}^{i \omega t}\left(1-\frac{4 r^{2}}{d^{2}}\right) .
$$

Substituting this expression into (9.3) gives

$$
V_{0}=\frac{\rho}{\mu} \frac{R_{\mathrm{c}} d^{2}}{16} \alpha_{\mathrm{ext}}
$$

As a result, the flow velocity amplitude $V_{0}$ defined in (9.6) is valid when

$$
\alpha_{\mathrm{ext}}<\frac{3200 \mu^{2}}{\rho^{2} d^{3} R_{\mathrm{c}}} .
$$

Following (9.6), to achieve a sensitive angular acceleration sensor, we need a fluid with a high density $\rho$ and low viscosity $\mu$, to obtain a relatively large fluid flow velocity. Furthermore, the channel should have a large diameter $d$ and the sensor benefits from a large system radius $R_{\mathrm{c}}$. 


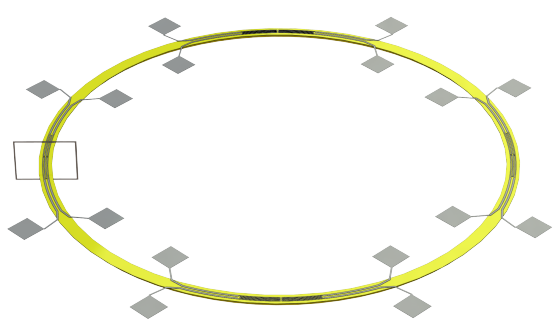

(a) Overview.

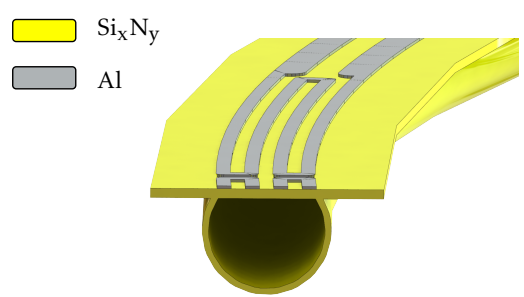

(b) Channel close-up.

Figure 9.2: Schematic view of the bio-inspired angular accelerometer with the heaters on top of the circular channel.
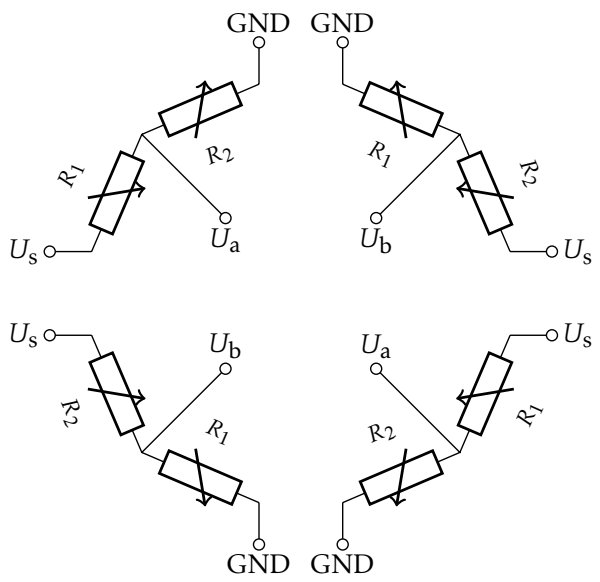

Figure 9.3: Schematical overview of the bridge configuration.

\subsubsection{Design}

The design of the angular accelerometer is based on the fabrication process of the micro Coriolis mass flow sensor, developed earlier in our group (Haneveld et al., 2010). The accelerometer (schematically shown in figure 9.2) consists of a tube with a diameter of $40 \mu \mathrm{m}$, whereas the diameter of the fully circular channel is designed to be $5.5 \mathrm{~mm}$. Further, two inlets are designed to fill the tube with a fluid, which is in our case water.

For measurement of the flow velocity, thermal read-out principles are used. Therefore, eight resistive elements are distributed along the circular channel. By applying a voltage over these resistors, heat is transferred to the fluid. In case of a fluid flow, the heat distribution will change and a change in resistance results (Lammerink et al., 1993). 
The circular channel is supplied with eight equally distributed resistive elements for thermal measurement of the fluid flow velocity (figure 9.3). In case of a fluid flow, heat will be transferred from resistor $R_{1}$ to resistor $R_{2}$ or vice versa, depending on the direction of the fluid flow. Therefore, we can define the resistors $R_{1}$ and $R_{2}$ as:

$$
R_{1}=R_{0}-\Delta R, \quad R_{2}=R_{0}+\Delta R,
$$

where $R_{0}$ is the intrinsic resistance in absence of fluid flow and resistive heating. Then, by implementing a Wheatstone-like bridge configuration for measurement of $\Delta R$, the bridge voltage $U_{\mathrm{a}}$ and $U_{\mathrm{b}}$ are defined as:

$$
U_{\mathrm{a}, \mathrm{b}}=U_{\mathrm{s}}\left(\frac{R_{1,2}}{R_{1}+R_{2}}\right)=U_{\mathrm{s}}\left(\frac{1}{2} \pm \frac{\Delta R}{2 R_{0}}\right) .
$$

By using a differential amplifier, the change in resistance $\Delta R$ and thus the fluid flow velocity $U_{0}$ can be measured directly:

$$
U_{\text {diff }}=U_{\mathrm{a}}-U_{\mathrm{b}}=U_{\mathrm{s}}\left(\frac{\Delta R}{R_{0}}\right) .
$$

Notice that in this configuration only two of the four available bridge output voltages are used.

\subsection{Fabrication}

To fabricate the bio-inspired angular accelerometer, the fabrication process of the micro Coriolis mass flow sensor described by Haneveld et al. (2010), schematically shown in figure 9.4, is used. First, a layer of low-stress LPCVD silicon-rich silicon nitride (SiRN) is deposited on a highly p-doped silicon wafer (a). Using deep reactive ion etching (DRIE), fluid inlet/outlet holes are etched from the backside using a photoresist (PR) mask, whereas the SiRN layer on top acts as a stop layer. Next, a $1 \mu \mathrm{m}$ thick $\mathrm{SiO}_{2}$ layer is deposited using TEOS and afterwards removed from the top side of the wafer. A $50 \mathrm{~nm}$ layer of chromium is sputtered to create the centrelines of the channels. The pattern is then transferred into the nitride layer by reactive ion etching (RIE) and subsequently the channels are etched in the silicon using isotropic plasma etching by $\mathrm{SF}_{6}(\mathrm{~b})$.

The $\mathrm{SiO}_{2}$ layer and chromium mask are then removed and another SiRN layer is grown with a thickness of $1.8 \mu \mathrm{m}$ to form the channel walls and to seal the etch holes in the first nitride layer (c). A $10 \mathrm{~nm}$ layer of chromium and $200 \mathrm{~nm}$ layer of gold are sputtered (chromium serves as the adhesion layer for gold) and 

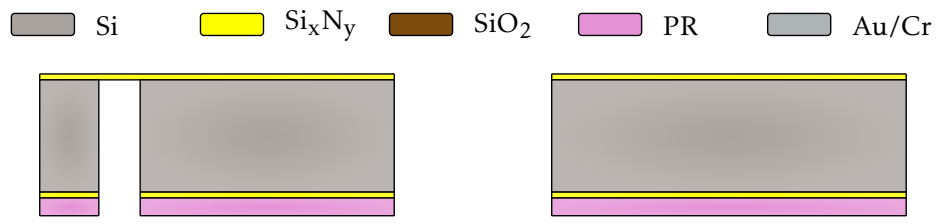

(a) Fluid inlet/outlet holes from backside using DRIE.
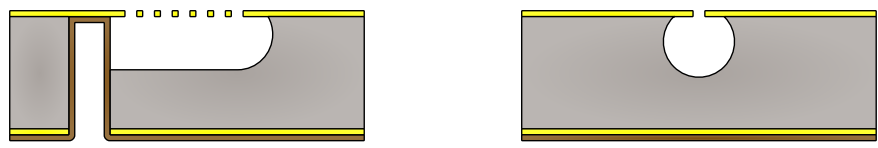

(b) Channel etching by isotropic etching of silicon.
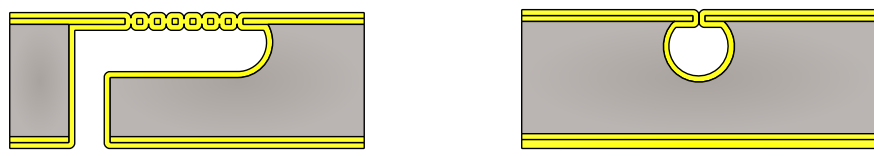

(c) Formation of channel walls and hole sealing by deposition of $\operatorname{Si}_{\mathrm{x}} \mathrm{N}_{\mathrm{y}}$.
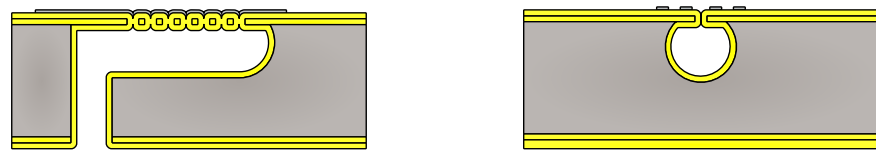

(d) Sputtering and patterning of electrodes $(\mathrm{Au} / \mathrm{Cr})$.
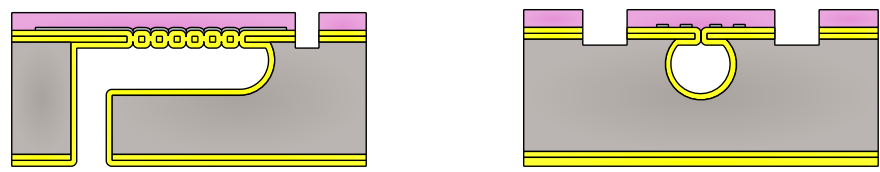

(e) Opening of release windows by RIE.
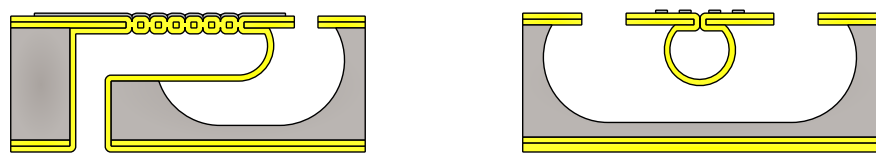

(f) Release of device by isotropic etching of silicon.

Figure 9.4: Schematic view of the fabrication process. Left: through-wafer cross-section along the length of the tube. Right: through-wafer cross-section of the tube.

patterned to create the metal electrodes for thermal read-out (d). To thermally isolate the channels from the silicon bulk, release windows are created by reactive ion etching (RIE) of the SiRN layer (e). Then, the structure is released by isotropic etching of silicon using $\mathrm{SF}_{6}(\mathrm{f})$. A fabricated bio-inspired angular accelerometer is shown by the SEM image in figure 9.5. 


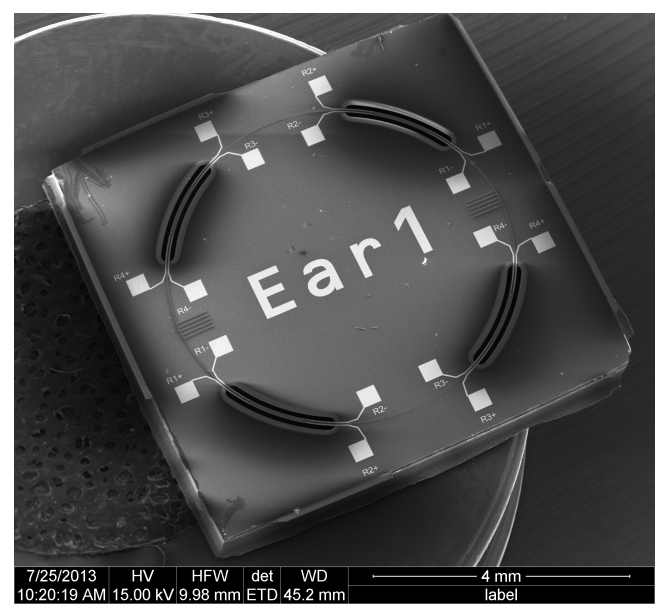

Figure 9.5: SEM image of the fabricated sensor.

\subsection{Experimental}

\subsubsection{Setup}

The accelerometer was tested using the rotational setup shown in figure 9.6. A small wheel is driven and connected by a lever with length $L$ to a large wheel, on which the bio-inspired vestibular system is mounted. By driving the small wheel with a constant angular velocity the large wheel will show harmonic angular accelerations with constant amplitude and a frequency depending on the angular velocity of the small wheel. The latter is driven by a stepper motor with a constant angular velocity $\omega$. For angles $\theta$ with amplitudes smaller than about $30^{\circ}$, the motion of the wheel can be considered sinusoidally. Consequently, the angular acceleration $\alpha(t)$ of the big wheel becomes

$$
\alpha(t) \approx-\omega^{2} \eta \sin (\omega t)
$$

where $\eta$ is a geometrical constant that depends on $R, H, G_{\mathrm{x}}, G_{\mathrm{y}}$ and $L$.

\subsubsection{Measurements}

To demonstrate the sensing capability of our bio-inspired angular acceleration sensor, the setup shown in figure 9.7 was used and experiments where performed for rotational frequencies within a range of $7-14 \mathrm{~Hz}$. The applied voltage $U_{\mathrm{s}}$ was generated sinusoidally using a Stanford SR 830 lock-in amplifier with its 


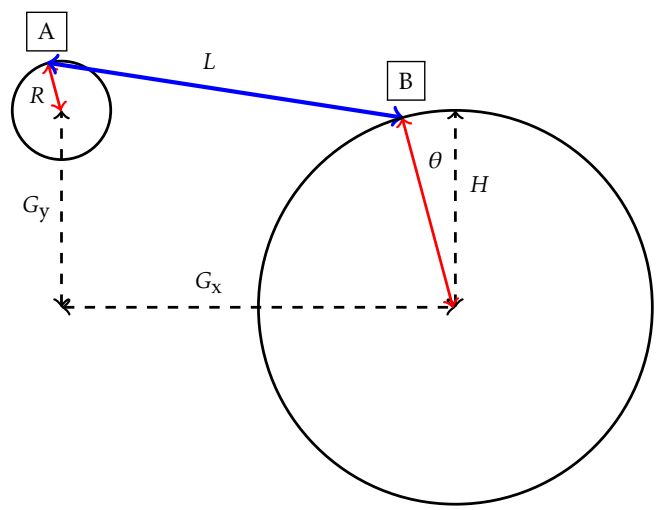

Figure 9.6: Schematical overview of the rotational setup.

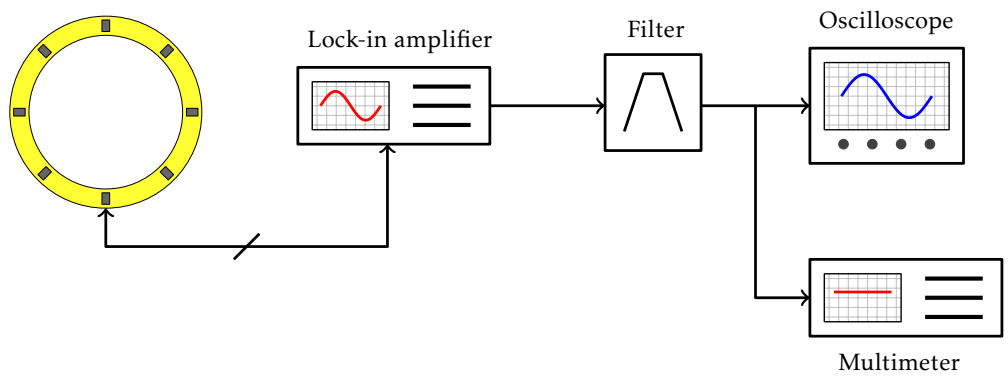

Figure 9.7: Experimental setup for measurement of the output bridge voltage.

frequency set to $50 \mathrm{kHz}$ and the amplitude to $0.7 \mathrm{~V}$. The output of the bridge configuration was measured differentially using the lock-in amplifier. Its output was demodulated by setting the amplifier time constant to $3 \mathrm{~ms}$ and the roll-off to $12 \mathrm{~dB}$. The resulting envelope was then band-pass filtered using a Stanford SR 650 filter system with its band-pass set to $1-20 \mathrm{~Hz}$, in order to improve the signal-to-noise ratio, and amplified with a gain of $20 \mathrm{~dB}$. The filtered output was monitored using an oscilloscope (Agilent DSO1024A) and its rms-value is measured using a multimeter (Keithley 2001). Using the geometrical properties of the rotational setup, the angular acceleration amplitude was calculated for every used frequency. The obtained results for the measured rms-voltage are shown in figure 9.8, together with a linear regression fit.

As we observe, the sensor's response is in good agreement with the expected linear response. The calculated full-scale error is found to be about $3.9 \%$, with the full-scale set at approximately $2 \times 10^{5 \circ} \mathrm{s}^{-2}$. To verify the sensor's response to angular accelerations, we changed the medium inside the tube from water to 


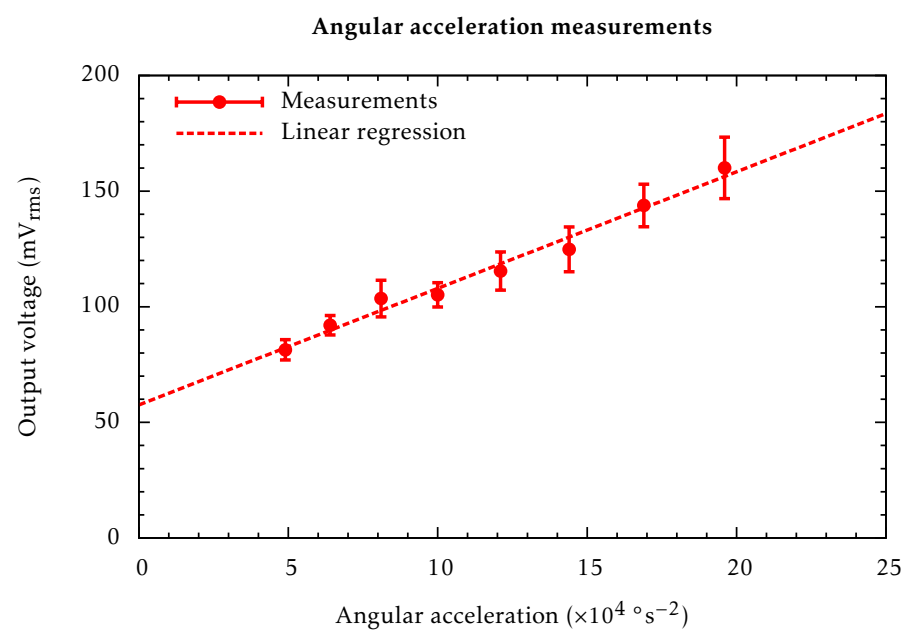

Figure 9.8: Measured response versus angular acceleration amplitudes.

air. As a result, the output voltage dropped significantly and no clear sinusoidal waveform was observed when performing measurements identical to those shown in figure 9.8.

\subsection{Discussion}

The measured range of angular acceleration amplitude is $5 \times 10^{4}-2 \times 10^{5 \circ} \mathrm{s}^{-2}$. These values are roughly comparable to commercially available angular accelerometers (STMicroelectronics, 2002; Endevco, 2009). However, by considering the lowest measured angular acceleration amplitude, the bio-inspired angular accelerometer turns out to be far less sensitive than the semicircular channel sensory system of both humans and monkeys. Groen and Jongkees (1948) presented results indicating that humans can measure angular accelerations down to $0.5-2^{\circ} \mathrm{s}^{-2}$, and Fernández and Goldberg (1971) showed that the monkey's semicircular channel system responds to angular accelerations down to $5^{\circ} \mathrm{s}^{-2}$.

The aim of this work is to demonstrate the concept of an angular acceleration sensor with a fully circular fluid-channel. Future steps include optimization of the sensor. Especially the heater's geometrical design requires attention for this purpose, for which we expect that the sensor's responsivity towards angular accelerations can be significantly improved. Namely, the current heaters are designed based upon the heater design of channel-based flow sensors developed earlier in our group, which has proven its suitability for thermal read-out. 


\subsection{Conclusions}

An angular accelerometer based on the semicircular channels of the vestibular system has been developed. The accelerometer consists of a water-filled tube, wherein the fluid flow velocity is measured thermally as a representative for the external angular acceleration. First measurements show a linear response for angular acceleration amplitudes up to $2 \times 10^{5 \circ} \mathrm{s}^{-2}$.

\section{References}

Arms, S. W. and Townsend, C. P. (2003). MEMS based angular accelerometer. Patent. US 0047002.

Damiano, E. R. and Rabbitt, R. D. (1996). A singular perturbation model of fluid dynamics in the vestibular semicircular canal and ampulla. J. Fluid Mech., 307:333-372.

Endevco (2009). Model 7302BM4 - Piezoresistive angular accelerometer.

Fernández, C. and Goldberg, J. M. (1971). Physiology of peripheral neurons innervating semicircular canals of the squirrel monkey. II. Response to sinusoidal stimulation and dynamics of peripheral vestibular system. J. Neurophysiol., 34(4):661-675.

Groen, J. J. and Jongkees, L. B. W. (1948). The threshold of angular acceleration perception. J. Physiol., 107:1-7.

Haneveld, J., Lammerink, T. S. J., de Boer, M. J., Sanders, R. G. P., Mehendale, A., Lötters, J. C., Dijkstra, M., and Wiegerink, R. J. (2010). Modeling, design, fabrication and characterization of a micro Coriolis mass flow sensor. J. Micromech. Microeng., 20:125001.

Lammerink, T. S. J., Tas, N. R., Elwenspoek, M., and Fluitman, J. H. J. (1993). Micro-liquid flow sensor. Sens. Act. A, 37-37:45-50.

Li, J., Fang, J., Du, M., and Dong, H. (2013). Analysis and fabrication of a novel MEMS pendulum angular accelerometer with electrostatic actuator feedback. Microsyst. Technol., 19:9-16.

Munson, B. R., Young, D. F., and Okiishi, T. H. (2006). Fundamentals of fluid mechanics. John Wiley \& Sons, Inc., USA, fifth edition. 
Nasiri, S. S., Yaralioglu, G. G., Seeger, J., and Taheri, B. (2011). Vertically integrated 3-axis MEMS angular accelerometer with integrated electronics. Patent. US 7934423.

Peng, X. F., Peterson, G. P., and Wang, B. X. (1994). Frictional flow characteristics of water flowing through rectangular microchannels. Exp. Heat Transf., 7(4):249264.

Ploechinger, H. (2004). Sensor for detecting a rotational movement or an angular acceleration. Patent. US 6722199.

STMicroelectronics (2002). LIS1R02 (L6671). Angular accelerometer.

Vega, R., Alexandrov, V. V., Alexandrova, T. B., and Soto, E. (2008). Mathematical model of the cupula-endolymph system with morphological parameters for the axolotl (ambystoma tigrinum) semicircular canals. Open Med. Inform. J., 2:138-148.

Yakushin, S. B., Raphan, T., Suzuki, J.-I., Arai, Y., and Cohen, B. (1998). Dynamics and kinematics of the angular vestibulo-ocular reflex in monkey: effects of canal plugging. J. Neurophysiol., 80(6):8077-8099. 

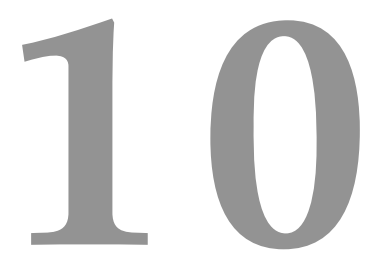

\section{Performance assessment of flow sensing hairs}

\subsection{Introduction}

A leap of faith is at the core of the biomimetic approach. It is based on a priori analogies and often times primarily visual impressions. It pervades the entire field of biomimetic design, despite valiant trials to move from art to science (Vincent et al., 2005) and explains the lavishing photographs, books and brochures picturing the living and the artefact side by side. Surprisingly, the engineering concept of a figure of merit (FoM) is hardly used in the biomimetic field: a recent survey using Web of Science found a maximum of a dozen of papers making quantitative comparisons between biomimetic devices and their biological templates using such metrics. When done, the results are somewhat disappointing at first. For example, such comparison between biomimetic flapping wing systems and birds or insects showed that animals have generally lower values of FoM (Mayo and Leishman, 2010). In that study, as well as in a theoretical study on osmotic actuation inspired by plants (Sinibaldi et al., 2013), the choice of the criteria used for building the FoM was a key step because the evolutionary pressures at play in

This chapter is based on "Performance assessment of bio-inspired sensor systems: flow sensing MEMS hairs" by H. Droogendijk, J. Casas, T. Steinmann, and G. J. M. Krijnen, in preparation for Nat. Commun., (2014). 
the real biological system are usually unknown. They are often inferred backwards from the performance, an approach where tautology is never far. Furthermore, the link between the optimal design of one compound, such as a single hair, and the fitness of the animal is seldom made (Dangles et al., 2005). In other words, what are the consequences for a cricket of producing suboptimal hairs in terms of survival and reproductive output? Of geckos harbouring setae which have suboptimal adherence properties? A naturalistic point of view would weigh in the frequency of the different substrate types on which geckos are living in the wild as well as their behaviour while walking (escaping danger, searching for mates, sun bathing, or running after prey). Such information is unfortunately nearly absent for most biomimetic systems. It is exemplary that the biological importance in determining plant fitness of the properties of the hooks so famous for being the template for the Velcro tissue are still unknown today. Finally, chosen criteria may be interdependent, making the further choice of the functional form of the figure of merit, beyond its ingredients, another key step.

Despite these caveats, we value the concept of an FoM because it forces us to declare unambiguously (i) the criteria to use and their functional relationship, (ii) the way to measure them both in the artefact and on the organism (iii) the targeted performance, (iv) the achieved gap between the organism and the artefact, (v) the level of improvement and (vi) the trade-off between metrics. Thus, the results of comparison between nature and technology contain important and valuable lessons. While more theoretical work on such issues is certainly warranted, the engineering world has its own technical conditions in terms of material properties, energy consumption, signal analysis etc. so that the number of possible scenarios for computing FoMs is very large, if not endless. Thus, an alternative experimental route, based on real measurements of built devices of varying properties and real organisms, consists in defining an FoM made of several criteria spanning a wide range of possible variables of importance in the biological system. In this paper, such a procedure is presented, and applied on bio-inspired flow sensors, inspired by the cricket's capability of sensing airflows using long, tiny hairs.

\subsubsection{Bio-inspired flow sensors}

Crickets are capable of sensing low-frequency flows by using mechanoreceptive sensory hairs to obtain information about the environment and avoid e.g. predator attacks. These so-called filiform hairs, which are situated on the back of the cricket's body on two appendices called cerci, are able to sense airflows with velocity amplitudes down to $30 \mu^{-1} \mathrm{~s}^{-1}$ (Shimozawa et al., 1998). Each filiform hair is lodged in a socket, guiding the hair to move in a preferred direction. When 
subjected to airflow, the neuron is fired upon rotation of the hair base (Fig. 10.1a). The measurement of such tiny airflows combined with the fact that these hairs work at the thermal noise level, which is an impressive performance (Shimozawa et al., 2003), is the main reason why engineers and biologists have marveled at these filiform hairs for so long. Another and related performance is the small hair amplitude required to trigger a neural response, of the order of $10^{-5}-10^{-4} \mathrm{rad}$ (Shimozawa et al., 2003).

Taking the cricket cercal hair sensors as a source of inspiration, several research groups have worked on the development of artificial counterparts for airflow measurements by exploiting MEMS technology (Ozaki et al., 2000; Chen et al., 2007; Wang et al., 2007; Sadeghi et al., 2011; Tao and Yu, 2012). In our work, hair-sensor inspired flow-sensors for measurement of (tiny) ac-airflows have been designed and fabricated (Dijkstra et al., 2005). Evolved fabrication methods and designs (figure 10.1b) have led to electronic-noise limited performance, enabling the detection and measurement of flow velocities in the range of sub- $\mathrm{mm} \mathrm{s}^{-1}$ while retaining a bandwidth on the order of $1 \mathrm{kHz}$ (Bruinink et al., 2009).

For all the existing MEMS cricket-inspired flow sensory systems developed, none of these shows equal or better performance compared to the cricket. To illustrate this, consider the experimental investigation for the responsivity, which is the normalized angular amplitude versus frequency, for both crickets and MEMS hair flow sensors shown in figure 10.1c. We observe that the cricket clearly shows a much larger angular amplitude for a given airflow velocity amplitude and for a broad range of frequencies. Though, a better performance compared to the cricket's flow sensory system has been in fact attained by human technology some time ago: the MEMS Microflown devices are able to measure speeds as low as $50 \mathrm{~nm} \mathrm{~s}^{-1}$ (de Bree, 2003) and laser Doppler anemometry and allied optical means are in fact outperforming the animal. The cost of such technological performance is however high: the technology is bulky, requires a lot of energy and a handling care that is much more delicate than the insect shows for its sensory hairs. They live in rough environments and do not seem to care much about chocks, mud or dust, and occasionally loose one or two hairs. Thus, it is the seemly incompatibility between the frugality in the design of the sensors and their fantastic performance that is exciting the minds of the engineers and biologists.

However, quantifying the performance of hair flow sensors based on the normalized angular amplitude is using only one metric. Other metrics for quantifying performance can be defined, and from a judiciously chosen combination of metrics an FoM can be defined and evaluated. In this paper, five of such metrics are presented for quantifying the performance of hair flow sensors, as well as a figure of merit. These are all numerically evaluated for both the cricket and the MEMS 


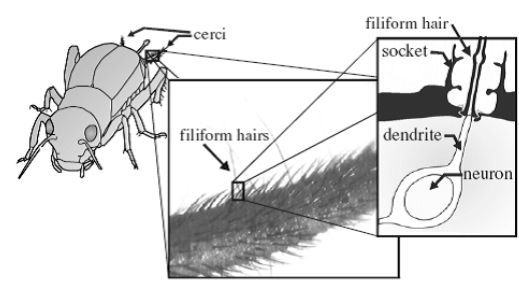

(a) Flow perception by crickets using mechanoreceptor filiform hairs.

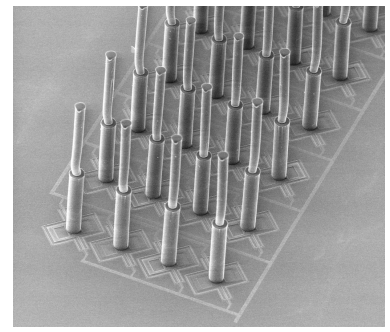

(b) Biomimetic hair flow sensors fabricated using MEMS technology.

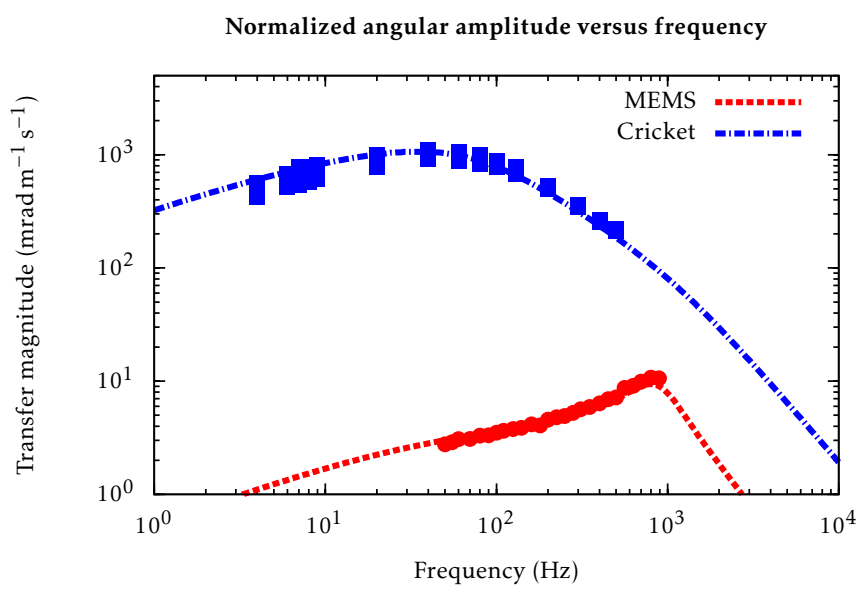

(c) Responsivity as function of frequency for the natural and man-made hairs. Crickets hairs (blue) are $800-900 \mu \mathrm{m}$, MEMS hairs (red) are $800 \mu \mathrm{m}$ long. Points represent experimental values extracted from (Shimozawa et al., 1998) and (Droogendijk et al., 2012)), while the fitted curves are based on (10.28).

Figure 10.1: From cricket filiform hairs to MEMS hair flow sensors.

sensors, and important insights can be gained from the results.

The organisation of this chapter is as follows. Section 10.2 starts with a theoretical model of the hair flow sensor, which is required for the definition of the performance metrics treated in section 10.3. Then, the evaluation of all performance metrics are given in section 10.4 and a comparison is made between the cricket and MEMS-based hair flow sensor performance. In section 10.5, the followed analysis and outcome for quantifying the performance of hair flow sensors is discussed, together with other aspects like the definition and impact of the chosen figure of merit. 


\subsection{Hair mechanical model}

The natural and MEMS hairs can be considered as a flow-driven inverted pendulum (figure 10.2a). Understanding this system requires us to both understand the flow-hair interaction as well as the mechanical response to the flow-induced torque on the hairs. This analysis has been successfully and thoroughly described by e.g. Shimozawa et al. (1998), Humphrey and Barth (2008) and Bathellier et al. (2012). For completeness, here we primarily reiterate these models and cast them in a formalism suitable to our analyses, while adding an original contribution considering mechanical impedance at the end of the section.

In the mechanical model, $S$ is the torsional stiffness, $R$ and $D$ are damping terms, $J$ and $C / \omega$ are contributions to the moment of inertia, and $T(t)$ is the torque acting on the hair by the viscous forces due to the airflow. The various parts constitute a second order mechanical system and exhibit corresponding behaviour with resonance and a mechanical roll-off proportional to $1 / \omega^{2}$ beyond resonance (figure 10.1c), in which $\omega$ is the flow frequency.

The flow susceptible hair is subjected to an oscillatory flow $v(t)$ with a given far field amplitude $V_{0}$ and angular frequency $\omega$. Assuming that the flow $v(t)$ is oscillating over a flat surface the no-slip boundary condition gives rise to the height $y$-dependent velocity profile (Panton, 1996; Steinmann et al., 2006) ${ }^{1}$ :

$$
v(y, t)=V_{0} e^{j \omega t}\left(1-e^{-\beta y} e^{-j \beta y}\right),
$$

where $\beta$ is proportional to the reciprocal of the boundary layer thickness $\delta_{b}$, with $v$ the kinematic viscosity:

$$
\beta=\sqrt{\frac{\omega}{2 v}} .
$$

The expression for $v(y, t)$ is written as a phasor with an amplitude $V_{\mathrm{y}}$ and phase shift $\zeta_{\mathrm{y}}$ :

$$
v_{\mathrm{y}}(t)=V_{\mathrm{y}} e^{j\left(\omega t+\zeta_{\mathrm{y}}\right)},
$$

where

$$
V_{\mathrm{y}}=V_{0} \kappa(y), \quad \kappa(y)=\sqrt{1+e^{-2 \beta y}-2 e^{-\beta y} \cos (\beta y)},
$$

and

\footnotetext{
${ }^{1}$ In this work, we use complex notation and take the observable quantities implicitly to be the respective real parts.
} 


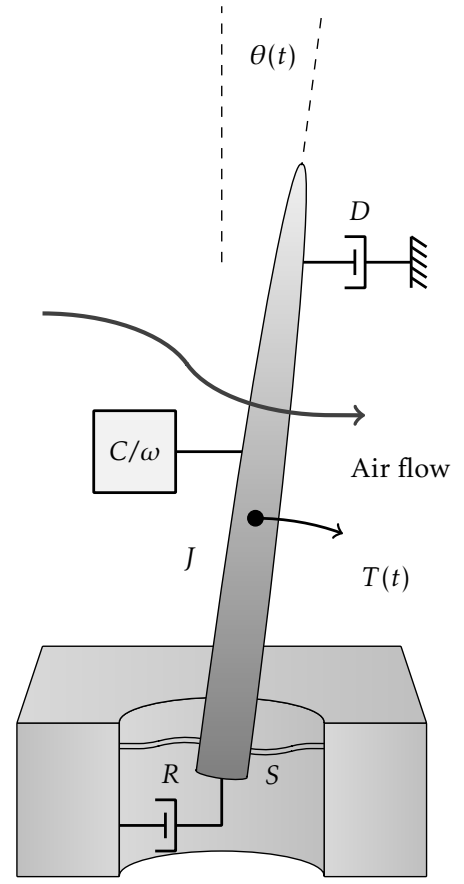

(a) Model of a flow sensing hair.

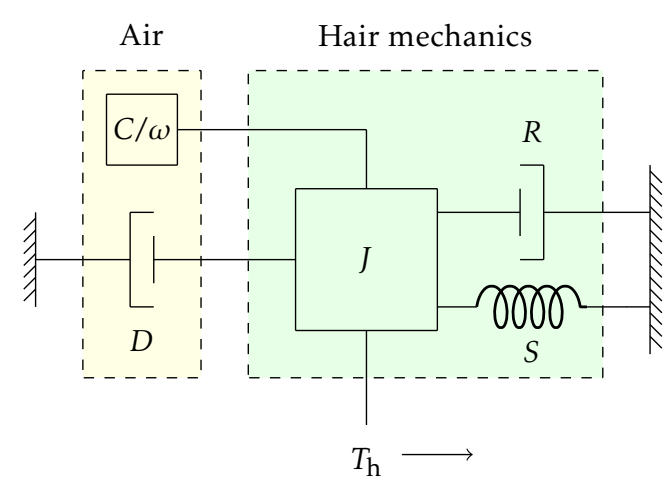

(b) Mechanical impedance implementation.

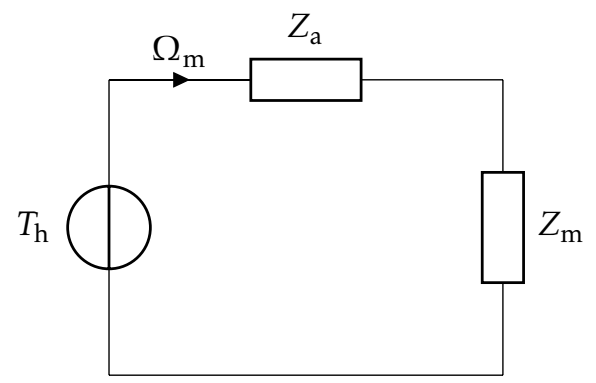

(c) Simple circuit representation of the impedances in the airflow-hair-sensor interaction.

Figure 10.2: Second order hair mechanical system. See text for the definition of the variables.

$$
\zeta_{\mathrm{y}}=\arctan \left(\frac{e^{-\beta y} \sin (\beta y)}{1-e^{-\beta y} \cos (\beta y)}\right) .
$$

With the velocity profile known, the forces exerted on the hair are calculated by Stokes' mechanical impedance $Z_{\mathrm{S}}$ as derived for a cylinder subject to oscillatory 
movement (Stokes, 1851). The relationship between the flow velocity $V$ and the drag force $F$ per unit length can be expressed as (Williams and Hussey, 1972; Shi and Sader, 2010):

$$
Z_{\mathrm{S}}=\frac{F}{V}=\pi \rho\left(\frac{d}{2}\right)^{2} \omega \Gamma(\omega),
$$

in which $\Gamma(\omega)$ is called the hydrodynamic function and given by

$$
\Gamma(\omega)=j\left[1+\frac{4 K_{1}(\sqrt{j \psi})}{\sqrt{j \psi} K_{0}(\sqrt{j \psi})}\right]
$$

where $K_{0}$ and $K_{1}$ are modified Bessel functions of the second kind and $\psi$ is the Stokes parameter:

$$
\psi=\frac{\omega}{v}\left(\frac{d}{2}\right)^{2}
$$

Notice that for $\psi \ll 1$ the mechanical impedance $Z_{S}$ given in (10.6) reduces by a series expansion to the expression used by Shimozawa et al. (1998) and Humphrey et al. (1993):

$$
Z_{\mathrm{S}} \approx 4 \pi \mu G+j\left[\pi \rho\left(\frac{d}{2}\right)^{2} \omega-\frac{\pi^{2} \mu G}{g}\right] .
$$

with $\mu$ and $\rho$ the dynamic viscosity and density of air respectively and $d$ the hair diameter. In these equations $G, g$ and $s$ are dimensionless parameters:

$$
G=\frac{-g}{g^{2}+(\pi / 4)^{2}}, \quad g=\gamma+\ln (s), \quad s=\frac{d}{4} \sqrt{\frac{\omega}{v}}=\frac{\sqrt{\psi}}{2} .
$$

The dynamic drag force $F(t)$ can be expressed as

$$
F(t)=V_{0}\left|Z_{S}\right| e^{j\left(\omega t+\eta_{\mathrm{S}}\right)}, \quad \eta_{\mathrm{S}}=\arg \left(Z_{\mathrm{S}}\right) .
$$

The velocity profile of the oscillatory flow is given by (10.3), but to calculate the drag forces on the hair using Stokes's mechanical impedance, the relative velocity of the hair $V_{\text {ry }}$ is required, where $y$ is the position along the hair. Assuming the resulting hair movement to be harmonic the relative velocity is given by

$$
V_{\mathrm{ry}}(t)=V_{\mathrm{y}} e^{j\left(\omega t+\zeta_{\mathrm{y}}\right)}-j y \Theta_{\mathrm{m}} \omega e^{j\left(\omega t+\phi_{\mathrm{m}}\right)},
$$


where $\Theta_{\mathrm{m}}$ is the hair angular deflection amplitude and $\phi_{\mathrm{m}}$ its phase. Using Stokes's mechanical impedance, the resulting force per unit length on the flow susceptible hair is found:

$$
F_{\mathrm{y}}(t)=\left|Z_{\mathrm{S}}\right|\left[V_{\mathrm{y}} e^{j\left(\omega t+\zeta_{\mathrm{y}}+\eta_{\mathrm{S}}\right)}-j y \Theta_{\mathrm{m}} \omega e^{j\left(\omega t+\phi_{\mathrm{m}}+\eta_{\mathrm{S}}\right)}\right] .
$$

The torque $T(t)$ on the hair is calculated from the force per unit length $F(t)$ acting on the hair:

$$
T(t)=\int_{0}^{L} F_{\mathrm{y}}(t, y) y \mathrm{~d} y .
$$

As a result, the torque $T(t)$ can be written as

$$
T(t)=V_{0} e^{j \omega t}\left[\int_{0}^{L} \kappa(y) y\left|Z_{\mathrm{S}}\right| e^{j\left(\zeta_{\mathrm{y}}+\eta_{\mathrm{S}}\right)} \mathrm{d} y\right]-j \omega \Theta_{\mathrm{m}} e^{j\left(\omega t+\phi_{\mathrm{m}}\right)}\left[\int_{0}^{L}\left|Z_{\mathrm{S}}\right| y^{2} e^{j \eta_{\mathrm{S}}} \mathrm{d} y\right]
$$

or in a simplified form as

$$
T(t)=V_{0} e^{j \omega t}[A+j B]-j \omega \Theta_{\mathrm{m}} e^{j\left(\omega t+\phi_{\mathrm{m}}\right)}[D+j C],
$$

where

$$
\begin{array}{ll}
A=\int_{0}^{L} \kappa(y)\left|Z_{S}\right| y \cos \left(\zeta_{y}+\eta_{S}\right) \mathrm{d} y, & C=\int_{0}^{L}\left|Z_{S}\right| y^{2} \sin \left(\eta_{S}\right) \mathrm{d} y, \\
B=\int_{0}^{L} \kappa(y)\left|Z_{S}\right| y \sin \left(\zeta_{y}+\eta_{S}\right) \mathrm{d} y, & D=\int_{0}^{L}\left|Z_{S}\right| y^{2} \cos \left(\eta_{S}\right) \mathrm{d} y .
\end{array}
$$

Following Shimozawa et al. (1998), the total torque (10.16) must be inserted in the right hand side of the second order differential equation for the hair-rotation:

$$
J \frac{\mathrm{d}^{2} \theta(t)}{\mathrm{d} t^{2}}+R \frac{\mathrm{d} \theta(t)}{\mathrm{d} t}+S=T(t)
$$

with $S$ is the torsional stiffness, $R$ the torsional resistance and $J$ the moment of inertia. It is assumed that the hair is harmonically driven with frequency $\omega$, giving:

$$
\begin{aligned}
& -\omega^{2} J \Theta_{\mathrm{m}} e^{j\left(\omega t+\phi_{\mathrm{m}}\right)}+j \omega R \Theta_{\mathrm{m}} e^{j\left(\omega t+\phi_{\mathrm{m}}\right)}+S \Theta_{\mathrm{m}} e^{j\left(\omega t+\phi_{\mathrm{m}}\right)}= \\
& V_{0} e^{j \omega t}[A+j B]+\omega \Theta_{\mathrm{m}} e^{j\left(\omega t+\phi_{\mathrm{m}}\right)}[C-j D] .
\end{aligned}
$$


This expression can be simplified to

$$
\Theta_{\mathrm{m}} e^{j \phi_{\mathrm{m}}}\left[S-J \omega^{2}-C \omega+j \omega(R+D)\right]=V_{0}[A+j B] .
$$

Consequently, the hair angular deflection amplitude $\Theta_{\mathrm{m}}$ and phase $\phi_{\mathrm{m}}$ are found to be:

$$
\Theta_{\mathrm{m}}=\frac{V_{0} \sqrt{A^{2}+B^{2}}}{\sqrt{\left[S-J \omega^{2}-C \omega\right]^{2}+[(R+D) \omega]^{2}}},
$$

and

$$
\phi_{\mathrm{m}}=\arctan \left(\frac{B\left[S-J \omega^{2}-C \omega\right]-A[(R+D) \omega]}{A\left[S-J \omega^{2}-C \omega\right]+B[(R+D) \omega]}\right) .
$$

In these expressions, $A$ and $B$ are contributions to the flow-induced drag torque $T(t), D$ is the virtual added damping and $C / \omega$ is the virtual added air mass (see also figure 10.2a) (Humphrey et al., 1993; Shimozawa et al., 1998).

From (10.21) it clearly follows that the system's rotational angle amplitude $\Theta_{\mathrm{m}}$ is determined by both the mechanical parameters $(S, R$ and $J)$ and the airflow related parameters $(D$ and $C / \omega)$. Since the hair flow sensor is considered to be subjected to harmonic airflows with frequency $\omega$, the hair itself will exhibit harmonic motion with angular velocity $\dot{\theta}(t)$, which can be calculated by taking the derivative of $\Theta_{\mathrm{m}} e^{j\left(\omega t+\phi_{\mathrm{m}}\right)}$ with respect to time:

$$
\frac{\mathrm{d} \theta(t)}{\mathrm{d} t}=\Omega_{\mathrm{m}} e^{j\left(\omega t+\phi_{\mathrm{m}}\right)}, \quad \Omega_{\mathrm{m}}=j \omega \Theta_{\mathrm{m}} .
$$

As a consequence, the system may alternatively be described as a dynamical system in terms of state variables $(\theta$ and $T)$, their time-derivatives $(\dot{\theta}, \dot{T})$ and their constitutive relations given by compliances, inductances and resistances (Olson, 1943). In this description, the time-derivative of the extensive variable, in this case the angular velocity, is called the flow and the intensive variable, here the drag torque, the effort. The compliances $(S)$, inductances $(J$ and $C / \omega)$ and resistances $(R$ and $D)$ can be considered as impedances. To this end, (10.19) can be rewritten using the definition for $\dot{\theta}(t)$ shown in (10.23) to:

$$
T_{\mathrm{h}}(t)=\left[(R+D)+j\left(J \omega-\frac{S}{\omega}+C\right)\right] \frac{\mathrm{d} \theta(t)}{\mathrm{d} t},
$$

where $T_{h}(t)$ equals the hair torque as a result of the contributors to the flow- 
induced drag torque $A$ and $B$ :

$$
T_{\mathrm{h}}(t)=V_{0} e^{j \omega t}[A+j B] .
$$

From (10.24) follows that the torque $T_{\mathrm{h}}(t)$ consists of impedances by the airflow related parameters (due to Stokes's mechanical impedance) and the mechanical hair parameters. A schematic view of these impedances is given in figure $10.2 \mathrm{~b}$, which is an abstracted version of figure 10.2a. Generally, the hair with moment of inertia $J$ is suspended by a torsional spring $S$ and has a torsional resistance $R$. The air itself also contributes to the total torque balance, where $C$ can be considered as "air virtual mass" and $D$ as added air viscous damping. Now, the hair flow sensor system can be simply described by

$$
T_{\mathrm{h}}(t)=\left(Z_{\mathrm{a}}+Z_{\mathrm{m}}\right) \frac{\mathrm{d} \theta(t)}{\mathrm{d} t}
$$

where the airflow and mechanical contributions in terms of impedances $Z_{\mathrm{a}}$ and $Z_{\mathrm{m}}$ are separated (see also figure 10.2c). Consequently, the airflow impedance $Z_{\mathrm{a}}$ and the mechanical impedance $Z_{\mathrm{m}}$ are defined as:

$$
Z_{\mathrm{a}}=D+j C, \quad Z_{\mathrm{m}}=R+j\left(J \omega-\frac{S}{\omega}\right)
$$

\subsection{Performance metrics}

In general, the performance of a sensory system can be described by either independent metrics or a judiciously chosen combination of metrics by the definition of a so-called figure of merit. In this section, five independent metrics are defined to quantify the performance of the described hair flow sensory system: responsivity, power transfer, power efficiency, response time and detection threshold. Additionally, a sixth metric is defined, which is a figure of merit based on three of the listed independent metrics. These metrics are described in turn below.

\subsubsection{Responsivity}

A characteristic of a sensory system is its responsivity. In engineering, systems are often designed to have a high responsivity. We define the responsivity of the hair flow sensory system as the resulting rotational angle amplitude per flow velocity amplitude: 


$$
\frac{\Theta_{\mathrm{m}}}{V_{0}}=\frac{\sqrt{A^{2}+B^{2}}}{\sqrt{\left[S-J \omega^{2}-C \omega\right]^{2}+[(R+D) \omega]^{2}}},
$$

where the first equality is due to the linear response of the hair-sensors with respect to airflow in accordance with the analysis of section 10.2. Following the approach by Bathellier et al. (2012), an upper limit for the responsivity can be defined. Under the conditions that the system operates at its resonance frequency $\left(S-J \omega^{2}=0\right)$, the absence of (socket) damping $(R=0)$, negligible air virtual mass $(C \ll D)$ and the fact that $A^{2} \gg B^{2}$ (Shimozawa et al., 1998), the limit of responsivity will be

$$
\left.\frac{\Theta_{\mathrm{m}}}{V_{0}}\right|_{\text {limit }}=\frac{A}{D \omega},
$$

where the system is dimensioned to have its maximum at frequency $\omega$. Notice that the limit of responsivity is fully determined by the flow-torque interaction on the hair and the airflow frequency. The drag-torque contributor $A$ can be directly related to $D$ (Shimozawa et al., 1998):

$$
A=\frac{3 D}{2 L}|K(\omega, L)|
$$

where the unitless parameter $|K(\omega, L)|$ captures the influence of the substrate on the incoming flow (Bathellier et al., 2012). In our case, the values for $|K(\omega, L)|$ are found between 0 and 1 . Therefore, the maximum achievable responsivity for the hair flow sensory system becomes

$$
\left.\frac{\Theta_{\mathrm{m}}}{V_{0}}\right|_{\text {limit }}=\frac{3}{2} \frac{1}{L \omega},
$$

which simply contains the hair length $L$ and the airflow frequency $\omega$.

\subsubsection{Power transfer}

The second metric for characterization of the performance of hair flow sensors is how much power is transferred to the mechanical part of the hair sensory system compared to the total power in the system (see also figure 10.2b). The timeaveraged power $P_{\mathrm{t}}$ dissipated by the resistive part of the mechanical impedance $Z_{\mathrm{m}}$ is given by

$$
P_{\mathrm{t}}=\Omega_{\mathrm{rms}}^{2} \operatorname{ke}\left(Z_{\mathrm{m}}\right)=\frac{1}{2} \Omega_{\mathrm{m}}^{2} \operatorname{ke}\left(Z_{\mathrm{m}}\right),
$$


where $\left|T_{\mathrm{h}}\right|$ is the flow-induced torque amplitude and $\Omega_{\mathrm{m}}$ is the hair rotational angular velocity amplitude:

$$
\left|T_{\mathrm{h}}\right|=V_{0} \sqrt{A^{2}+B^{2}}, \quad \Omega_{\mathrm{m}}=\frac{\left|T_{\mathrm{h}}\right|}{\left|Z_{\mathrm{a}}+Z_{\mathrm{m}}\right|} .
$$

The transferred power $P_{\mathrm{t}}$ is maximum when the impedances $Z_{\mathrm{m}}$ and $Z_{\mathrm{a}}$ are matched, according to the maximum power transfer theorem (Cartwright, 2008), which requires:

$$
\operatorname{Ke}\left(Z_{\mathrm{m}}\right)=\operatorname{Ke}\left(Z_{\mathrm{a}}\right), \quad \operatorname{Im}\left(Z_{\mathrm{m}}\right)=-\operatorname{Im}\left(Z_{\mathrm{a}}\right),
$$

which implies

$$
R=D, \quad \omega^{2}=\frac{S}{J+C / \omega} .
$$

In case the impedances are matched, the maximum transferable power $P_{\max }$ can be calculated and is given by

$$
P_{\max }=\frac{\left|T_{\mathrm{h}}\right|^{2}}{8 D} .
$$

Now, we define the transferred power ratio $\lambda$ as the ratio between the power $P_{\mathrm{t}}$ present in the resistive part of the mechanical impedance $(R)$ and the power present in the total system in case of matched impedances $P_{\max }$ :

$$
\lambda=\frac{P_{\mathrm{t}}}{P_{\max }}=\frac{4 R D}{\left|Z_{\mathrm{a}}+Z_{\mathrm{m}}\right|^{2}},
$$

where the limit for maximum power transfer is simply

$$
\left.\lambda\right|_{\text {limit }}=1 .
$$

\subsubsection{Power efficiency}

Thirdly, power efficiency can be considered as a metric for sensor performance. The power efficiency $\eta$ can be defined as the ratio between the power dissipated by the resistance $R$ of the mechanical system to the power developed by the incoming airflow in the hair-system. The power dissipated in the resistance $R$ is given by (10.32) and the total power $P_{\text {tot }}$ input into the system is simply

$$
P_{\text {tot }}=\frac{1}{2}\left|T_{\mathrm{h}}\right| \Omega_{\mathrm{m}} .
$$


Using the previously defined impedances and (10.26), the power efficiency $\eta$ is defined as

$$
\eta=\frac{P_{\mathrm{t}}}{P_{\text {tot }}}=\frac{R}{\left|Z_{\mathrm{a}}+Z_{\mathrm{m}}\right|},
$$

Also here, the limit for maximum power efficiency is simply

$$
\left.\eta\right|_{\text {limit }}=1
$$

\subsubsection{Response time}

The performance of wind receptor hairs can also be determined against the metric of response time. Besides a power-efficient and sensitive sensory system, also the ability to quickly respond to a change in flow contributes to the performance of the system. The corresponding response time $\tau_{95}$ - a measure for agility - is calculated from the second order differential equation, describing the mechanical behaviour of the system in response to a torque step function. The general solution of a damped mass-spring system is

$$
\theta(t)=\Theta_{1} e^{-r_{1} t}+\Theta_{2} e^{-r_{2} t}
$$

where $\Theta_{1}$ and $\Theta_{2}$ need to be derived from initial conditions and $r_{1}$ and $r_{2}$ are found from

$$
r_{1,2}=\frac{\Gamma}{2} \pm \sqrt{\left(\frac{\Gamma}{2}\right)^{2}-\omega_{0}^{2}}=\frac{\Gamma}{2} \pm \omega_{0} \sqrt{\zeta^{2}-1},
$$

with $\Gamma$ and $\omega_{0}$ constants which can be expressed using the damping ratio $\zeta$ as

$$
\Gamma=\frac{R_{\mathrm{t}}}{J_{\mathrm{t}}}=2 \zeta \omega_{0}, \quad \omega_{0}^{2}=\frac{S}{J_{\mathrm{t}}} .
$$

Considering the presence of the air impedance in the total model, the total damping $R_{\mathrm{t}}$ and total moment of inertia $J_{\mathrm{t}}$ are given by

$$
R_{\mathrm{t}}=R+D, \quad J_{\mathrm{t}}=J+\frac{C}{\omega} .
$$

Since the quantities $C, D$ and $J_{\mathrm{t}}$ contain a frequency-dependency, these parameters are evaluated at resonance $(\omega=\sqrt{S / J})$ in this work. The general solution of $(10.42)$ is either real or complex. When the damping ratio $\zeta$ is equal or greater than one, the solution is real and the hair moves without oscillations towards the point of torque equilibrium, dominated by the mathematically slow moving term. When 
the damping ratio $\zeta$ becomes smaller than one, the square root term becomes imaginary and the hair shows overshoot towards its equilibrium, leading to a longer stabilization time. The response time $\tau_{95}$, indicated as the time to get stable within $95 \%$ of the end value, is approximated ${ }^{2}$ as:

$$
\tau_{95} \approx \begin{cases}\frac{3}{\zeta \omega_{0}} & \text { if } \zeta<0.5, \\ \frac{6 \zeta}{\omega_{0}} & \text { if } \zeta>1 .\end{cases}
$$

The best response time is obtained when $\zeta$ corresponds to a slightly underdamped system (Shinners, 1998). Consequently, the lower limit for the response time is found when:

$$
\left.\zeta\right|_{\text {limit }}=\frac{1}{2} \sqrt{2}
$$

\subsubsection{Detection threshold}

Another metric of performance for a sensory system is its signal-to-noise ratio (SNR). Generally, when the SNR is equal or higher than one, the system is able to detect the input signal. As a result, when the responsivity is given, the noise level of the system determines its detection threshold. To find the fundamental detection threshold of the flow susceptible hairs, the mechanical-thermal noise due to Brownian motion of molecules in the hair structure needs to be addressed (Gabrielson, 1993). The associated power spectral density of this (white) noise is given by

$$
\bar{T}_{\mathrm{n}}^{2}=4 k_{\mathrm{B}} T_{0} R_{\mathrm{t}},
$$

where $\overline{T_{\mathrm{n}}^{2}}$ is the squared equivalent noise torque, $k_{\mathrm{B}}$ is the Boltzmann's constant, $T_{0}$ is the ambient temperature and $R_{\mathrm{t}}$ is the total resistance given by (10.45). The mechanical transfer function $H(\omega)$ of the system determines the resulting mechanical noise power:

$$
H(\omega)=\frac{1}{-J_{\mathrm{t}} \omega^{2}+j R_{\mathrm{t}} \omega+S} .
$$

The noise equivalent angle $\Theta_{n}$ is found by integrating the noise power over the full spectrum and taking the square root:

\footnotetext{
${ }^{2} \mathrm{~A}$ precise mathematical solution is rather involved. A spline interpolation is used for connecting the two asymptotes smoothly and obtaining a good approximation, which is not shown here.
} 


$$
\Theta_{\mathrm{n}}=\sqrt{\int_{0}^{\infty} \overline{T_{\mathrm{n}}^{2}}|H(\omega)|^{2} \mathrm{~d} \omega} .
$$

The detection threshold $V_{\text {th }}$ is found by dividing the sensor's equivalent noise angle $\Theta_{n}$ by the amplitude of the rotational angle $\Theta_{m}$ at a far field flow amplitude $V_{0}$ of $1 \mathrm{~m} \mathrm{~s}^{-1}$, where the latter is required for normalization of (10.28):

$$
V_{\mathrm{th}}(\omega)=\frac{\Theta_{\mathrm{n}}}{\left.\Theta_{\mathrm{m}}(\omega)\right|_{V_{0}=1 \mathrm{~ms}^{-1}}} .
$$

The minimum achievable detection threshold is determined by both the torsional resistance $R$ and the responsivity of the hair sensory system. Namely, by reducing the noise and increasing the signal a better signal-to-noise ratio is obtained. The lowest thermal noise values occur when the hair Brownian motion is fully determined by the added damping by air $D$, since this constant depends on the hair geometry. Thus, letting $R=0$. Also, the best responsivity is achieved when the limit of (10.31) is fulfilled. Therefore, the threshold for the lowest measurable flow amplitude is defined as:

$$
\left.V_{\text {th }}(\omega)\right|_{\text {threshold }}=\frac{2}{3} \Theta_{\mathrm{n}} L \omega,
$$

with $\Theta_{\mathrm{n}}$ the equivalent noise angle $(R=0)$ :

$$
\Theta_{\mathrm{n}}=\sqrt{4 k_{\mathrm{B}} T_{0} \int_{0}^{\infty} \frac{D}{\left(S-J_{\mathrm{t}} \omega^{2}\right)^{2}+D^{2} \omega^{2}} \mathrm{~d} \omega .}
$$

\subsubsection{Figure of merit}

In engineering, figures of merit are frequently defined for particular materials or devices in order to determine their relative utility for an application. In our case, responsivity is of high importance in a sensory system, but also the time it takes for the system to respond to a change in airflow and a low detection threshold. Because the responsivity is by itself already covered in our definition of the detection threshold by (10.51), we eventually defined the figure of merit (FoM) for a hair flow sensory system as:

$$
\text { FoM }=\frac{1}{\text { Detection threshold } \times \text { Response time }},
$$

for which the response time and detection threshold have been defined previously, together with their physical limits. As a consequence of this FoM, the flow sensory 
system performs well by having a low detection threshold (able to measure tiny flows) and a short response time (fast system). There are numerous ways to define a FoM and we decided to leave out power metrics, for reasons given in section 10.5.1.

\subsection{Application of metrics}

Based on the described metrics, both the cricket cercal hair and the MEMS hair flow sensors are evaluated for varying values of the parameters listed in table 10.1. Since most mechanical parameters (e.g. $C$ and $D$ ) have a slight frequency-dependency, an airflow is considered with a frequency of $200 \mathrm{~Hz}$, which is a frequency within the cricket's sensing bandwidth (see figure 10.1c).

Also the differences between the cricket and the MEMS hair flow sensor system are listed by means of a so-called lead factor for the cricket. That is, how many times better the cricket e.g. performs on a given metric with respect to the MEMS sensor system. Notice that the detection threshold of the MEMS hair flow sensor is based here on thermal-mechanical noise only and does not represent the current state of performance, for which the detection threshold is $1.11 \mathrm{~mm} \mathrm{~s}^{-1}$ at $200 \mathrm{~Hz}$ due to limitations by electronic noise (Dagamseh et al., 2013). In practice, the current MEMS sensors perform worse when taking into account this measured detection threshold, which results in a current FoM-score that is about 4 times lower than the cricket.

The length of natural hairs varies in crickets from 100-1500 $\mu \mathrm{m}$ (Dangles et al., 2008; Miller et al., 2011). Hair length is furthermore a key variable in determining the hair's mechanical response and also one of the easiest parameters to vary in building MEMS. We therefore explored the performance of natural and MEMS hairs as function of their length.

Taking the allometric scaling property for cricket hairs by Shimozawa et al. (1998) into account (i.e. the quantities $S, R$ and $d$ all depend directly on the hair length $L$ ), the graphs from figure 10.3 (leftside) result upon varying the cricket hair length $L: 400 \mu \mathrm{m}, 800 \mu \mathrm{m}$ and $1600 \mu \mathrm{m}$. Further, in the same figure (right side) the impact of variations in hair length for the MEMS hair sensory system is shown (with the parameters $S, R$ and $d$ fixed, since allometric scaling does not apply for MEMS), and a comparison with the cricket's performance can be made. For both hair types, the physical limits for the various metrics are indicated by a horizontal dotted line. Since the response time is a frequency-independent quantity, it is shown with respect to the hair length $L$ in figure 10.4, together with its physical limit. Evaluation of the figure of merit for both cricket and MEMS is shown in figure 10.5, for which also the maximum physically achievable limit is 
Table 10.1: Characteristics of a cricket cercal hair (non-metrics data from (Shimozawa et al., 1998)) and a MEMS hair (non-metrics data from Droogendijk et al. (2012)) for a flow frequency of $200 \mathrm{~Hz}$. The lead factor indicates the rational difference between the cricket and the MEMS sensor system.

\begin{tabular}{lllll}
\hline Quantity & Symbol & Cricket & MEMS & Factor \\
\hline Hair parameters & & & & \\
\hline Hair length & $L$ & $800 \mu \mathrm{m}$ & $800 \mu \mathrm{m}$ & $\mathrm{n} / \mathrm{a}$ \\
Base diameter & $d$ & $7.01 \mu \mathrm{m}$ & $44 \mu \mathrm{m}$ & $0.16 \times$ \\
Density & $\rho$ & $1100 \mathrm{~kg} \mathrm{~m}^{-3}$ & $1200 \mathrm{~kg} \mathrm{~m}^{-3}$ & $0.92 \times$ \\
\hline Mechanical parameters & & & & \\
\hline Torsional stiffness & $S$ & $1.31 \times 10^{-11} \mathrm{Nm} \mathrm{rad}^{-1}$ & $9.5 \times 10^{-9} \mathrm{Nm} \mathrm{rad}^{-1}$ & $0.001 \times$ \\
Torsional resistance & $R$ & $1.55 \times 10^{-14} \mathrm{Nmsrad}^{-1}$ & $1.20 \times 10^{-12} \mathrm{Nm} \mathrm{sad}^{-1}$ & $0.013 \times$ \\
Added virtual damping & $D$ & $1.01 \times 10^{-14} \mathrm{Nmsrad}^{-1}$ & $1.83 \times 10^{-14} \mathrm{Nm} \mathrm{sad}^{-1}$ & $0.55 \times$ \\
Moment of inertia & $J$ & $2.44 \times 10^{-18} \mathrm{~kg} \mathrm{~m}^{3}$ & $3.12 \times 10^{-16} \mathrm{~kg} \mathrm{~m}^{3}$ & $0.008 \times$ \\
Added virtual mass & $C / \omega$ & $1.81 \times 10^{-18} \mathrm{~kg} \mathrm{~m}^{3}$ & $7.20 \times 10^{-18} \mathrm{~kg} \mathrm{~m}^{3}$ & $0.25 \times$ \\
\hline Metrics & & & & \\
\hline Responsivity & $\Theta_{\mathrm{m}} / V_{0}$ & $0.592 \mathrm{rad} \mathrm{m}^{-1} \mathrm{~s}^{-1}$ & $0.004 \mathrm{rad} \mathrm{m}^{-1} \mathrm{~s}^{-1}$ & $148 \times$ \\
Power transfer & $\lambda$ & 0.92 & 0.0014 & $657 \times$ \\
Power efficiency & $\eta$ & 0.59 & 0.149 & $4.0 \times$ \\
Response time & $\tau$ & $5.87 \mathrm{~ms}_{95}$ & $1.59 \mathrm{~ms}^{-1}$ & $0.27 \times$ \\
Detection threshold & $V_{\text {th }}$ & $73.7 \mu \mathrm{m} \mathrm{s}^{-1}$ & $352 \mu \mathrm{m} \mathrm{s}^{-1}$ & $4.8 \times$ \\
Figure of merit & FoM & $2.312 \times 10^{6} \mathrm{~m}^{-1}$ & $1.787 \times 10^{6} \mathrm{~m}^{-1}$ & $1.29 \times$ \\
\hline
\end{tabular}

indicated by a horizontal dotted line.

\subsection{Discussion}

Our model of the hair mechanics is based on the analysis by Shimozawa et al. (1998).We made substantial advance on the computation of impedances with both real and imaginary parts. The concept of impedance matching by equalling the real parts of the impedances $(R=D)$ had been mentioned earlier by Shimozawa et al. (1998) and Bathellier et al. (2012), but the imaginary parts $\left(\omega^{2}=S /[J+C / \omega]\right)$ were not available. As a result, a complete definition of the power metrics for both transfer and efficiency can now be made. The signal-to-noise ratio, and hence the sensitivity (expressions (10.48) to (10.53)), is also new and is valuable when comparing artificial and natural hairs. Notice that our performance analysis is applicable to hair flow sensors in general, and hence it can be used to e.g. flow sensors for underwater sensing (Humphrey and Barth, 2008). 
a) Responsivity - Cricket (allometric)

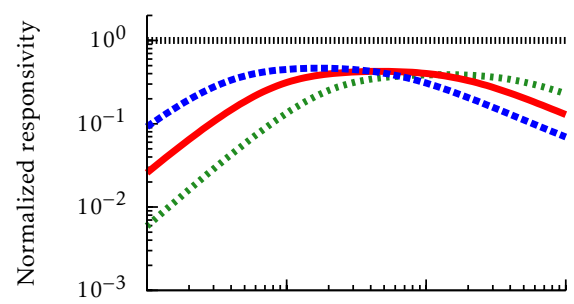

b) Power transfer - Cricket (allometric)

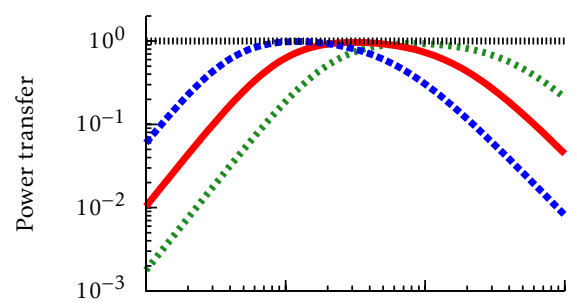

c) Power efficiency - Cricket (allometric)

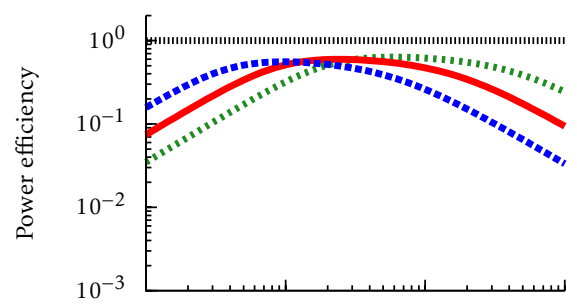

d) Detection threshold - Cricket (allometric)

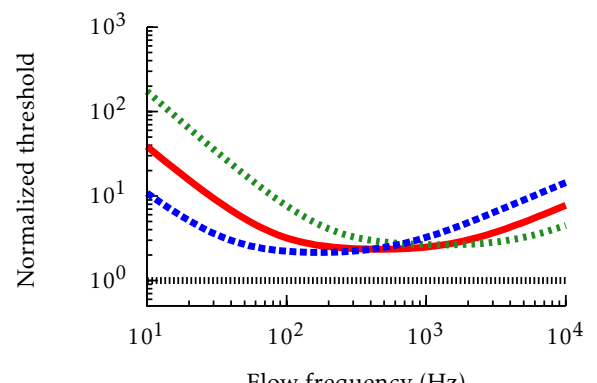

Flow frequency $(\mathrm{Hz})$

_ Original $(L) \quad \ldots \ldots, \quad$ Shorter hair $(0.5 L)$

เnแ!" Physical limit .... Longer hair $(2 L)$ a) Responsivity - MEMS

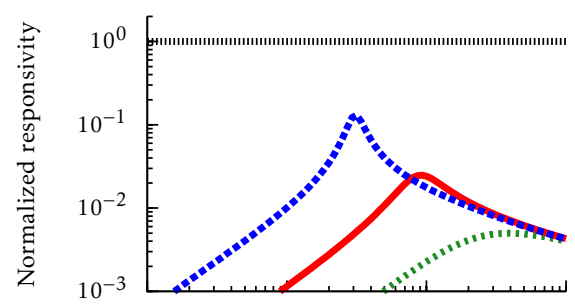

b) Power transfer - MEMS

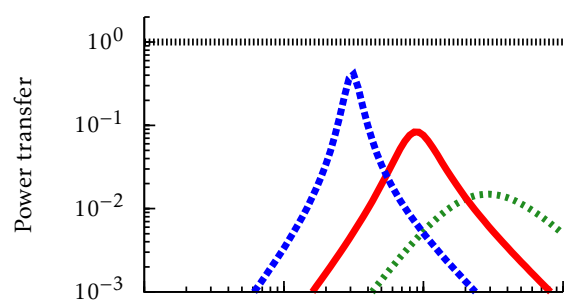

c) Power efficiency - MEMS

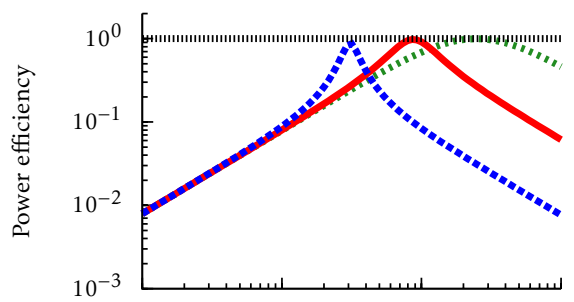

d) Detection threshold - MEMS

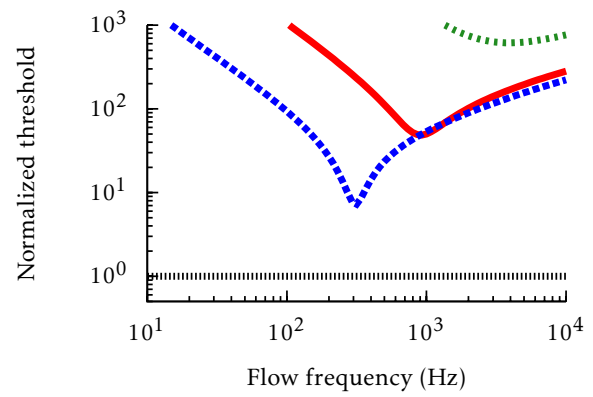

Figure 10.3: Performance metrics of natural and MEMS hairs of varying lengths as function of flow frequency. Both hairs are $800 \mu \mathrm{m}$ long. Metrics are normalized when possible. Allometric scaling among the hair's variables is applied to the cricket hair. 


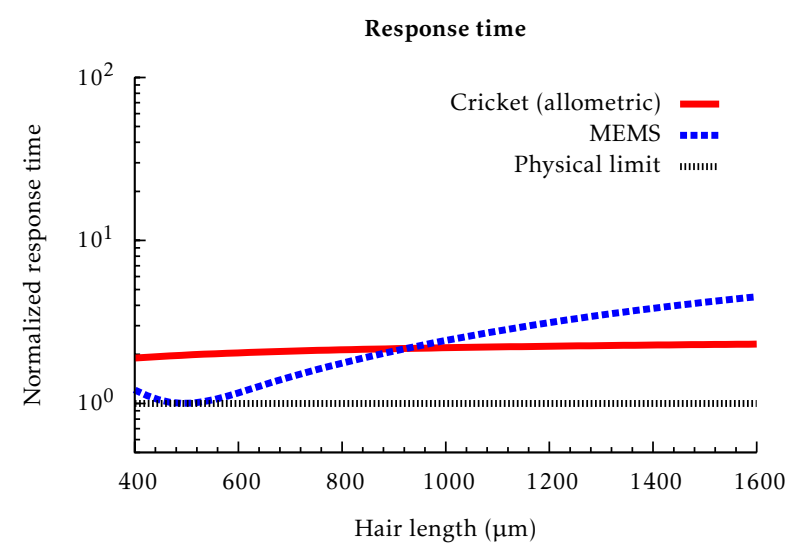

Figure 10.4: Response time as function of hair length for natural (red) and MEMS hairs (blue), normalized by the optimal response time $\left(\zeta=\frac{1}{2} \sqrt{2}\right)$. Allometric scaling is used for the cricket.

\subsubsection{Model evaluation and choice of performance metrics}

The considered linear second order mechanical model yields analytical solutions to all six metrics, a convenient and handy outcome. Also, the modelling of Humphrey et al. (1993), Shimozawa et al. (1998) and Bathellier et al. (2012) all produced results which were largely in line with experimental findings and over a reasonable large bandwidth as well. Though, for a more accurate description of the real mechanical behaviour it is possible that our linear mechanical model is incomplete and consequently needs to be adapted. For example, Shimozawa et al. (1998) propose to describe the torsional stiffness $S$ as a viscoelastic (Maxwell) element. Also, McConney et al. (2009) determined that, at low angular velocity, the motion of the hair was most accurately captured by a three-parameter viscoelastic model, which is a similar finding. Research for the Drosophila by Göpfert and Robert (2002) indicates that the torsional stiffness $S$ shows a non-linearity, indicating that a similar non-linearity in the torsional stiffness $S$ for the here described natural wind receptor hairs cannot be excluded.

The exact definitions of several metrics might impact the final outcome and need to be discussed. First, the role of power is not clear to us, hence our avoidance in using it to define the FoM. Considering the system's energy, a distinction can be made upon both power transfer $\lambda$ and power efficiency $\eta$. Maximum power efficiency occurs when the source impedance $Z_{\mathrm{a}}$ is minimized, transferring maximum power to the mechanical impedance $Z_{\mathrm{m}}$. However, the torque $T_{h}$ contributors parameters $A$ and $B$ both depend on the hair geometry, as do the 


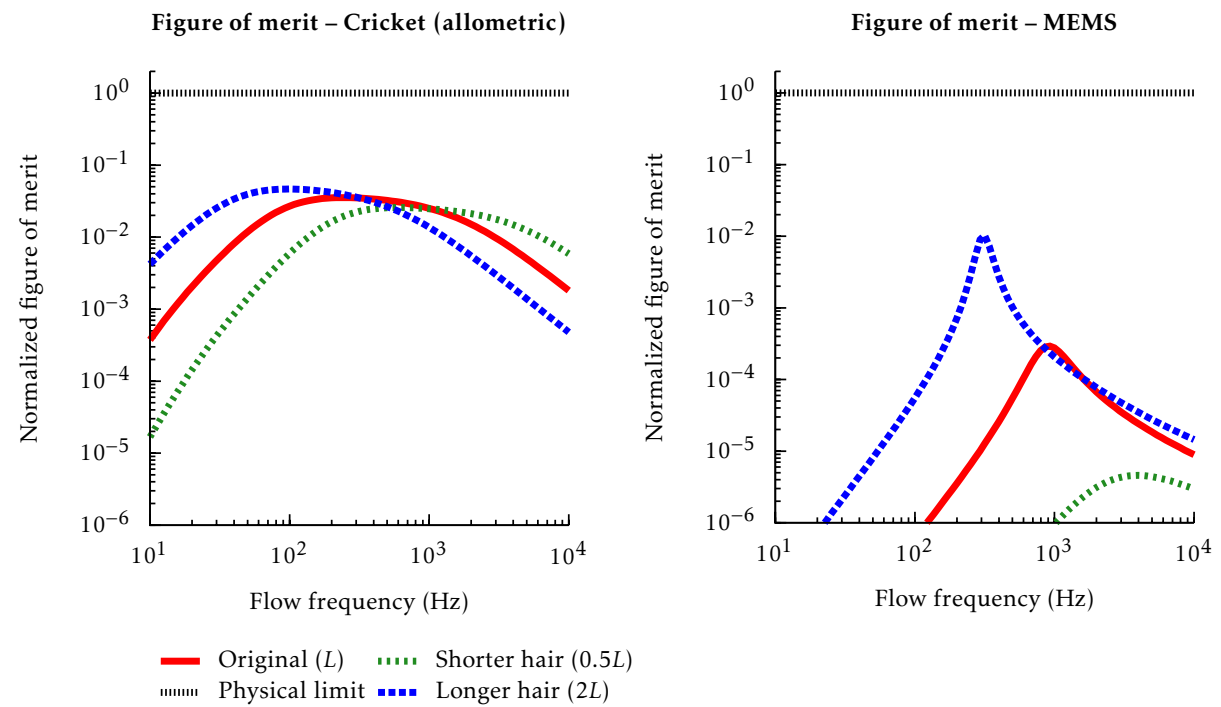

Figure 10.5: Figure of merit of natural and MEMS hairs of varying lengths as function of flow frequency, normalized by the physical limit. Allometric scaling among the hair's variables is applied to the cricket hair.

parameters $C / \omega$ and $D$ (see model building). This means that minimizing $D$ leads to a significant decrease of $T_{\mathrm{h}}$, thus less transferred power. Therefore, we think that maximum power transfer might be more important than maximum power efficiency. As for the cricket's hairs, it is possible that impedance matching plays a role in the cricket sensory system. For example, Keil (1997) implies that a good power transfer by impedance matching is desired for effective flow measurement. It is by contrast questionable whether a high power efficiency is present at all in crickets. As for MEMS hair flow sensors, improving power transfer and efficiency will give no benefits as they measure the rotational angle directly and not the transferred power. The power metrics can be useful for other MEMS sensor systems though, where the power has to be provided from a source external to the stimulus, e.g. the thermal-based MEMS Microflown devices (de Bree, 2003).

The response time is another metric which might be defined in varying ways. We defined it as the fastest possible stable output in response to a step function, which generally implies having a critically damped mechanical system as being the best performing system. As for the cricket, it has been found that their cercal hairs are indeed close to critically damped systems (Shimozawa et al., 1998; Bathellier et al., 2012). Thus, our definition of the response time seems in coherence with this observation. As for the MEMS hair flow sensor system, the angular amplitude 
is measured directly. Therefore, we defined the response time as being the ability to measure the airflow velocity both in a fast and non-ringing way.

\subsubsection{Unwanted cross-talk between sensory modalities and mul- tifunctional sensory systems}

The hair structures have been so far in all the published literature considered to be primarily responsive towards airflows. However, due to the very nature of the hair mechanical system the sensory system will also respond towards inertial effects due to external accelerations. The sensor system can therefore suffer from cross-talk when the sensor is not only subjected to airflow, but also to accelerations. This might be well the case during the chase-escape bouts between prey crickets and predator spiders in nature (Morice et al., 2013). This inertial responsivity affects the measurement of airflows and can potentially lead to a distortion in flow information due to this crosstalk. Since cricket and MEMS hair flow sensor are compared to each other, a comparison can also be made for the undesired inertial responsivity. We considered the torques acting on the hair due to airflow and due to inertial effects by external accelerations. To calculate the accelerationinduced torque acting on the hair, the analysis laid out in (Droogendijk et al., 2013) is used. Since the hair diameter plays a much stronger role in the acceleration induced torque $\left(\propto d^{2}\right)$ compared to the flow-induced torque ( $\left.\propto d^{1 / 3}\right)$ (Krijnen et al., 2007; Droogendijk et al., 2013), the exact hair geometry is additionally taken into account for both cricket hairs (Kumagai et al., 1998) and MEMS hairs (Droogendijk et al., 2012).

The ratio between flow-induced torque and acceleration-induced torque is taken as a measure. The calculated ratio of the normalized flow responsivity (torque per $\mathrm{ms}^{-1}$ ) versus the normalized acceleration responsivity (torque per $\mathrm{m} \mathrm{s}^{-2}$ ) is generally about 40 times larger for a cricket $800 \mu \mathrm{m}$ hair compared to a MEMS hair of the same length (Droogendijk et al., 2013). The hair diameter $d$ plays therefore a pivotal role and the MEMS hair flow sensor performs notably worse due to its relative thickness. As a result, to minimize the inertial responsivity for a hair flow sensor, the system clearly benefits from having a hair with a small diameter. By contrast, changing the hair length $L$ has negligible impact on the ratio of responsivities, since both flow-induced torque and acceleration-induced torque scale with $L^{2}$ (Krijnen et al., 2007; Droogendijk et al., 2013). It is unknown at this stage if crickets might use these hairs as inertial sensing devices, as they also possess other sensors, assumed to be dedicated to that purpose, the clavate sensillae (Murphey, 1981; Sakaguchi and Murphey, 1983; Horn and Bischof, 1983). What is here described as a nuisance might be turned as an advantage if the 
sensor is multifunctional. The bias induced by such cross-talk remains however to take into account. These considerations, stemming from the engineering, device characterization point of view, about cross-talk between sensory modalities are totally new to the field of hair based flow sensing, both technologically and in the natural systems. Generally, cross-talk between sensory modalities is seldomly studied in natural systems (it is for example totally absent in (Smith, 2008)).

\subsubsection{Improving MEMS flow sensors: time to abandon nature- inspired design?}

The figure of merit, hardly used in biomimetic and nature-inspired technology, is a composite single number. A high degree of subjectivity exists in the way such index is built, the choice of functional relationship between its constituents, being linear, quadratic or else. Nevertheless, a single number gives the engineer a handy estimate of overall performance of a system or device, in particular in relative terms. The performances of cricket and MEMS-based hair flow sensory systems, as based upon our figure of merit, indicate that the cricket's flow sensor is performing over 4 times better than the current MEMS hair flow sensor. In contrast to the MEMS sensors, the cricket's flow sensor approaches the physical limit for all metrics, leaving substantial room for improvement for MEMS hairs.

Changing the hair length is among the most obvious possibilities to improve at least some of the metrics. Since the flow-drag torque interaction constants $A$ and $B$ depend strongly on $L$ and the constants $C / \omega$ and $D$ are relatively small for a MEMS hair flow sensor, increasing its hair length will also give a much higher responsivity and lower detection threshold. The lower threshold is caused by the fact that $A$ and $B$ are much more affected than the hair mechanical system $H(\omega)$ and thus a slight change in thermal mechanical noise. For both cricket and MEMS, a disadvantage of increasing $L$ is however the increased response time, which makes the system responding slower to changes in flow. Kant and Humphrey (2009) investigated the responsivity of cricket hairs towards airflow pulsations and indeed found that short hairs are significantly more responsive than long hairs to rapid changes in airflow. Thus, what we seem to gain in performance through selected aspects is lost through others. Cummins and Gedeon (2012) derived similar conclusions for hairs with different lengths by modelling and estimating their response towards airflow pulsations. Changing the hair geometry in order to minimize the added virtual damping $D$ is complex, since this will also affect the flow-induced drag torque and generally leads to a smaller rotational angular amplitude.

By considering the outcome of the metric evaluation (figures 10.3 and 10.5), we 
observe that the cricket's hairs — in contrast to the MEMS hairs - operate close to their physical limit, which is in agreement with the observations by Bathellier et al. (2012). Although increasing the hair length $L$ of the MEMS sensors will help to bring their performance closer to their limits, the resonance frequency of the system reduces and the resonance peak sharpens. To increase the normalized MEMS sensor's performance for a broad range of frequencies, similar to the cricket, the limit definitions shown in (10.31), (10.47) and (10.53) hold the key. That is, the airflow-related parameters $A$ and $D$ need to be taken as a starting point, and from that the hair geometry $(L, d)$ and the system parameters $S$ and $R$ should be realized such that $D \gg R$, and the damping ratio $\zeta$ approaches the value of a critically damped system. Notice that this optimization towards the optimum may be limited due to practical constraints by the exploited MEMS technology.

One promising aspect we identified through our approach as appropriate target for technological improvement is the signal-to-noise ratio. Two ways can be envisioned to achieve this. First, we propose a reduction in MEMS sensor's noise floor by electrostatically lowering the torsional stiffness $S$. It is demonstrated by Droogendijk et al. (2012) that lowering the sensor's stiffness can lead to a reduction of more than $30 \%$ of the detection threshold if the sensor's thermal noise is small compared to the noise of other sources. Another, compatible, way to reduce noise would be through further reduction of the electronics noise. Ultimately attaining the cricket's performance in terms of signal-to-noise ratio would bring the lead factor down to about one, a large improvement. Further improvement can be expected in making thinner MEMS hairs, a task which is however technologically challenging, as well as using stochastic resonance, parametric amplification and other signal processing designs. Most if not all of these solutions imply however leaving the nature-inspired approach of the early developmental stages to the benefit of producing a complete, integrated functional sensor system.

\section{References}

Bathellier, B., Steinmann, T., Barth, F. G., and Casas, J. (2012). Air motion sensing hairs of arthropods detect high frequencies at near-maximal mechanical efficiency. J. R. Soc. Interface, 9(71):1131-1143.

Bruinink, C. M., Jaganatharaja, R. K., de Boer, M. J., Berenschot, J. W., Kolster, M. L., Lammerink, T. S. J., Wiegerink, R. J., and Krijnen, G. J. M. (2009). Advancements in technology and design of biomimetic flow-sensor arrays. In Proc. MEMS 2009, pp. 152-155. 
Cartwright, K. V. (2008). Non-calculus derivation of the maximum power transfer theorem. Tech. Interface.

Chen, N., Tucker, C., Engel, J., Yang, Y., Pandya, S., and Liu, C. (2007). Design and characterization of artificial haircell sensor for flow sensing with ultrahigh velocity and angular sensitivity. J. Microelectromech. S., 16:999-1014.

Cummins, B. and Gedeon, T. (2012). Assessing the mechanical response of groups of arthropod filiform flow sensors. In Frontiers in sensing. Springer.

Dagamseh, A. M. K., Bruinink, C. M., Wiegerink, R. J., Lammerink, T. S. J., Droogendijk, H., and Krijnen, G. J. M. (2013). Interfacing of differentialcapacitive biomimetic hair flow-sensors for optimal sensitivity. J. Micromech. Microeng., 23:035010.

Dangles, O., Magal, C., Pierre, D., Olivier, A., and Casas, J. (2005). Variation in morphology and performance of predator-sensing system in wild cricket populations. J. Exp. Biol., 208(3):461-468.

Dangles, O., Steinmann, T., Pierre, D., Vannier, F., and Casas, J. (2008). Relative contributions of organ shape and receptor arrangement to the design of cricket's cercal system. J. Comp. Physiol. A, 194:653-663.

de Bree, H.-E. (2003). An overview of microflown technologies. Acta Acust. United Ac., 89:163-172.

Dijkstra, M., van Baar, J. J. J., Wiegerink, R. J., Lammerink, T. S. J., de Boer, J. H., and Krijnen, G. J. M. (2005). Artificial sensory hairs based on the flow sensitive receptor hairs of crickets. J. Micromech. Microeng., 15:S132-S138.

Droogendijk, H., Bruinink, C. M., Sanders, R. G. P., Dagamseh, A. M. K., Wiegerink, R. J., and Krijnen, G. J. M. (2012). Improving the performance of biomimetic hair-flow sensors by electrostatic spring softening. J. Micromech. Microeng., 22(6):065026.

Droogendijk, H., de Boer, M. J., Sanders, R. G. P., and Krijnen, G. J. M. (2013). Design, fabrication and characterisation of a biomimetic accelerometer inspired by the cricket's clavate hair. In Proc. IEEE Sensors 2013, pp. 167-170, Baltimore, MD, United States.

Gabrielson, T. B. (1993). Mechanical thermal noise in micromachined acoustic and vibration sensors. IEEE Trans. Electron. Devices, 40:903-909. 
Göpfert, M. C. and Robert, D. (2002). The mechanical basis of Drosophila audition. J. Exp. Biol., 205:1199-1208.

Horn, E. and Bischof, H.-J. (1983). Gravity reception in crickets: The influence of cereal and antennal afferences on the head position. J. Comp. Physiol. A, 150:93-98.

Humphrey, J., Devarakonda, R., Iglesias, I., and Barth, F. (1993). Dynamics of arthropod filiform hairs. I. Mathematical modeling of the hair and air motions. Phil. Trans.: Bio. Sc., 340:423-444.

Humphrey, J. A. C. and Barth, F. (2008). Medium flow-sensing hairs: biomechanics and models. Adv. Insect Phys., 34:1-80.

Kant, R. and Humphrey, J. A. C. (2009). Response of cricket and spider motionsensing hairs to air flow pulsations. J. R. Soc. Interface, 6(6):1047-1064.

Keil, T. A. (1997). Functional morphology of insect mechanoreceptors. Microsc. Res. Techniq., 39:506-531.

Krijnen, G., Floris, A., Dijkstra, M., Lammerink, T., and Wiegerink, R. (2007). Biomimetic micromechanical adaptive flow-sensor arrays. Proc. SPIE, 6592:65920F.

Kumagai, T., Shimozawa, T., and Baba, Y. (1998). The shape of wind-receptor hairs of cricket and cockroach. J. Comp. Physiol. A, 183:187-192.

Mayo, D. B. and Leishman, J. G. (2010). Comparison of the hovering efficiency of rotating wing and flapping wing micro air vehicles. J. Am. Helicopter Soc., 55(2):25001.

McConney, M. E., Schaber, C. F., Julian, M. D., Eberhardt, W. C., Humphrey, J. A., Barth, F. G., and Tsukruk, V. V. (2009). Surface force spectroscopic point load measurements and viscoelastic modelling of the micromechanical properties of air flow sensitive hairs of a spider (Cupiennius salei). J. R. Soc. Interface, 6(37):681-694.

Miller, J. P., Krueger, S., Heys, J. J., and Gedeon, T. (2011). Quantitative characterization of the filiform mechanosensory hair array on the cricket cercus. PLoS ONE, 6:e27873.

Morice, S., Pincebourde, S., Darboux, F., Kaiser, W., and Casas, J. (2013). Predatorprey pursuit-evasion games in structurally complex environments. Integr. Comp. Biol., ict061:1-13. 
Murphey, R. K. (1981). The structure and development of a somatotopic map in crickets: The cereal afferent projection. Dev. Biol., 88:236-246.

Olson, H. F. (1943). Dynamical analogies. D. van Nostrand Company, New York.

Ozaki, Y., Ohyama, T., Yasuda, T., and Shimoyama, I. (2000). An air flow sensor modeled on wind receptor hairs of insects. In Proc. MEMS 2000, pp. 531-536, Miyazaki, Japan.

Panton, R. L. (1996). Incompressible flow. Wiley.

Sadeghi, M., Peterson, R., and Najafi, K. (2011). Micro-hydraulic structure for high performance biomimetic air flow sensor arrays. In Tech. Digest - IEDM, 6131638, pp. 29.4.1-29.4.4.

Sakaguchi, D. S. and Murphey, R. K. (1983). The equilibrium detecting system of the cricket: physiology and morphology of an identified interneuron. J. Comp. Physiol. A, 150:141-152.

Shi, Y. and Sader, J. E. (2010). Lattice Boltzmann method for oscillatory Stokes flow with applications to micro- and nanodevices. Phys. Rev. E, 81:036706.

Shimozawa, T., Kumagai, T., and Baba, Y. (1998). Structural scaling and functional design of the cercal wind-receptor hairs of cricket. J. Comp. Physiol. A, 183:171186.

Shimozawa, T., Murakami, J., and Kumagai, T. (2003). Cricket wind receptors: thermal noise for the highest sensitivity known. In Sensors and sensing in biology and engineering, pp. 145-159. Springer.

Shinners, S. M. (1998). Modern control system theory and design. John Wiley \& Sons, Inc., $2^{\text {nd }}$ edition.

Sinibaldi, E., Puleo, G. L., Mattioli, F., Mattoli, V., Di Michele, F., Beccai, L., Tramacere, F., Mancuso, S., and Mazzolai, B. (2013). Osmotic actuation modelling for innovative biorobotic solutions inspired by the plant kingdom. Bioinspir. Biomim., 8(2):025002.

Smith, C. U. M. (2008). Biology of sensory systems. John Wiley \& Sons, Inc., $2^{\text {nd }}$ edition.

Steinmann, T., Casas, J., Krijnen, G., and Dangles, O. (2006). Air-flow sensitive hairs: boundary layers in oscillatory flows around arthropod appendages. J. Exp. Biol., 209:4398-4408. 
Stokes, G. G. (1851). On the effect of the internal friction of fluids on the motion of pendulums. Trans. Cambr. Phil. Soc., 9:1-141.

Tao, J. and Yu, X. (2012). Hair flow sensors: from bio-inspiration to biomimicking-a review. Smart Mater. Struct., 21:113001.

Vincent, J. F. V., Bogatyreva, O., Pahl, A.-K., Bogatyrev, N., and Bowyer, A. (2005). Putting biology into TRIZ: a database of biological effects. Creativity and Innovation Management, 14(1):66-72.

Wang, Y.-H., Lee, C.-Y., and Chiang, C.-M. (2007). A MEMS-based air flow sensor with a free-standing microcantilever structure. Sensors, 7:2389-2401.

Williams, R. E. and Hussey, R. G. (1972). Oscillating cylinders and Stokes' paradox. Phys. Fluids, 15(12):2083-2088. 
REFERENCES 


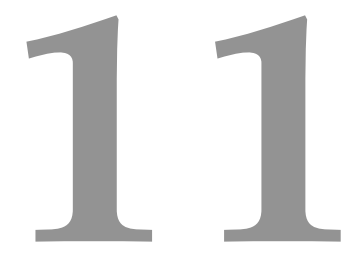

\section{Conclusions and outlook}

\subsection{Conclusions}

This thesis focuses on bio-inspired MEMS flow and inertial sensors, for which the research can be roughly divided into three parts. First, cricket-inspired hair flow sensors are addressed, for which electromechanical mechanisms are exploited to improve and optimize their performance. Second, an extensive characterization of these flow sensors is described and a thorough performance analysis based on metrics is made. Third, several sensors for inertial sensing are presented, inspired by different biological sources of inspiration.

\subsubsection{Performance optimization}

Exploiting the electrostatic nature of the capacitive read-out structures of our cricket-inspired hair flow sensors, the performance of these sensors can be improved and adapted to the environment. Applying a dc-bias voltage on the capacitors of our biomimetic hair flow sensors gives both an increase in responsivity for frequencies within the sensor's bandwidth and lowers the sensory threshold on implementation of electrostatic spring softening (ESS). Also a high frequency ac-bias voltage was shown to improve the responsivity of the system. Generally, 
we can detect lower flow velocities by adapting our flow sensors by applying bias voltages to the sensor's capacitive structures. By applying a dc-bias voltage on ESH capacitive structures, the bandwidth is increased at the cost of reduced responsivity of the hair flow sensor.

Changing the dc-bias voltage to an ac-bias voltage, non-resonant parametric amplification and filtering have been demonstrated in our hair flow sensors. By selecting appropriate values for the ac-bias voltage, selective gain and filtering is achieved. The responsivity to the incoming airflow can be improved by $20 \mathrm{~dB}$, while having large selectivity with respect to non-matched frequency signals.

On application of an appropriate sinusoidal voltage on the capacitor plates, upconversion of the flow information is achieved when the flow frequency is much lower than the voltage frequency resulting in electromechanical amplitude modulation (EMAM). It is demonstrated that EMAM can improve the measurement performance at low frequencies, in case of limitations within the measurement setup. This method can be applied equally well to transients as to harmonic signals. In the latter case threshold limits can be improved up to 25 times, whereas in the transient measurements it is demonstrated that tiny signals, which are below the noise-levels in the measurement's base-band, can be revealed well when upconverted to higher frequencies.

Exploiting the mechanism of stochastic resonance (SR) in a voltage-controlled MEMS-slider, the signal-to-noise ratio can be increased by adding white noise. We implemented stochastic resonance by controlling the strength of positiondependent capacitive wells by a dc-bias voltage, operating the device in push-pull mode by electrostatic actuation and adding a judicious amount of white noise to one of the actuation comb drives. We showed that the use of SR allows for detection of sub-threshold forces. Further, it is demonstrated that the noise bandwidth has a clear impact on the required optimal noise strength. The mechanism of SR is implemented for several types of periodic waveforms. The fundamental frequency can be resolved in all cases, but the type of waveform (sine, triangle or square) turns out to be irresolvable using SR.

\subsubsection{Hair flow sensors}

For extensive characterization of MEMS hair flow sensors, three different types of oscillatory flow setups are investigated. A loudspeaker, a vibrating sphere and a standing wave tube all have their own characteristics and depending on the application the appropriate source needs to be chosen. By combining insights using all three setups more information is obtained about the sensor response then when using a specific source solely. 
In quantifying the performance of MEMS hair flow sensors and comparing it to their source of inspiration, the cricket cercal mechano-sensitive hairs, the question as to which metrics to use becomes unavoidable. For both natural and biomimetic flow sensitive hair sensory systems, power transfer, power efficiency, responsivity, response time and detection threshold are attributes of the sensor performance. These five metrics are described, modelled and evaluated for both cricket and MEMS hair sensors. In general, cricket flow sensors perform not only better than the MEMS hair sensors, but also operate close to their physical limits. Additionally, the metrics responsivity, response time and detection limit are captured into a single number by means of a figure of merit. On evaluation of this figure of merit, the cricket performs two orders of magnitude better than their MEMS counterpart. Though, our MEMS hair flow sensors are still among the best artificial hair flow sensors.

\subsubsection{Bio-inspired sensors}

A biomimetic accelerometer has been developed and fabricated using surface micromachining and SU-8 lithography, inspired by the clavate hair system of the cricket. This MEMS hair-based accelerometer has a resonance frequency of $320 \mathrm{~Hz}$, a detection threshold of $0.10 \mathrm{~m} \mathrm{~s}^{-2}$ and a dynamic range of more than $35 \mathrm{~dB}$. Further, the accelerometer exhibits low responsivity to airflow, clear directivity and a bias instability of $5 \times 10^{-3} \mathrm{~m} \mathrm{~s}^{-2}$. The accelerometer can be further optimized by increasing the hair diameter, and it principally allows for adaptation to the environment by exploiting the mechanism of ESS.

Inspired by the fly's haltere, a biomimetic gimbal-based gyroscope has been designed, fabricated and partially characterized. Design rules for gyroscopes show that the haltere-based gyroscope have evolved towards large sensing bandwidth and a fast response, rather than a high responsivity. Measurements on the MEMS sensors indicate an excitable gyroscope with a (drive mode) resonance frequency of about $550 \mathrm{~Hz}$ and a damping ratio of 0.9 . The response to externally applied angular rates is not demonstrated due to the very small induced displacements in the sense mode, which is mainly caused by the high damping ratio. In contrast to the fly, our haltere-based gyroscope is physically limited to drive angles of about $0.5^{\circ}$ compared to the fly $\left(85^{\circ}\right)$.

An angular accelerometer based on the semicircular channels of the vestibular system has been developed. The accelerometer consists of a water-filled tube, wherein the fluid flow velocity is measured thermally as a representative for the external angular acceleration. First measurements show a linear response for angular acceleration amplitudes up to $2 \times 10^{5} \mathrm{~s}^{-2}$. 


\subsection{Outlook}

The implementation of electrostatic spring softening is demonstrated for our cricket-inspired hair flow sensors. However, the theory we have used to derive the expressions for the ESS-effect has been based entirely on the nature of energy buffering transducers. In more detail, an equivalent form can be derived for any energy buffering transducer, thus not only electrostatic, but also piezoelectric, electromagnetic and other types of transduction. Therefore, controlling the system's torsional stiffness is not limited to our hair flow sensors, but can be applied to numerous sensor types to implement e.g. non-resonant parametric amplification (NRPA) or electromechanical amplitude modulation (EMAM).

The design and fabrication of several hair-based bio-inspired sensors are described in this thesis: a hair flow sensor to measure flow, an accelerometer to measure external acceleration and a gyroscope to measure angular rates. A next step in the biomimetic approach is the combination of hair-based sensors to arrive at a multi-sensor system in which sensor fusion can take place. Also, other types of bio-inspired hair-based sensors offer a potential for integration within hair-based multi-sensor systems, like IR-light sensing (Schmitz et al., 2012) and tactile sensing (Murphey, 1985).

Over the past years, much research has been done on biomimetic hair flow sensors. Despite all effort and the many important scientific results on hair-based flow sensing, it remains a challenge to engineers and scientists to realize the artificial counterpart of the cricket hair flow sensor system with equal or better performance. Currently, the cricket performs still much better on several aspects (e.g. responsivity and detection threshold), provoking the question as to how to 'beat the cricket'. To this end, look for improvement of the design of the mechanical suspension, investigate the use of more compliant materials and consider the (partial) use of different or new technology.

\section{References}

Murphey, R. K. (1985). A second cricket cercal sensory system: bristle hairs and the interneurons they activate. J. Comp. Physiol. A, 156:357-367.

Schmitz, H., Soltner, H., and Bousack, H. (2012). Biomimetic infrared sensors based on photo-mechanic infrared receptors in pyrophilous ("fire-loving") insects. IEEE Sensors J., 12(2):281-288. 


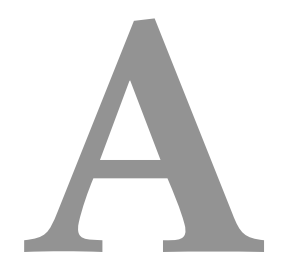

\section{Oscillating airflow and Stokes' drag coefficient}

Assuming that the flow with velocity $v(t)$ is oscillating over a flat surface, the no-slip boundary condition gives rise to the height $z$-dependent velocity profile (Panton, 1996):

$$
v(t)=V_{0} \sin (\omega t)-V_{0} e^{-\beta z} \sin (\omega t-\beta z),
$$

where $\beta$ is proportional to the reciprocal of the boundary layer thickness, with $v$ the kinematic viscosity $(\beta=\sqrt{\omega /(2 v)})$. Using trigonometric identities, expression (A.1) is written as a sinusoidal function with an amplitude $V_{\mathrm{z}}$ and phase shift $\zeta_{\mathrm{Z}}$ :

$$
v_{\mathrm{z}}(t)=V_{\mathrm{z}} \sin \left(\omega t+\zeta_{\mathrm{z}}\right)
$$

where

$$
V_{\mathrm{z}}=V_{0} \sqrt{1+e^{-2 \beta z}-2 e^{-\beta z} \cos (\beta z)},
$$

and 


$$
\zeta_{\mathrm{z}}=\arctan \left(\frac{e^{-\beta z} \sin (\beta z)}{1-e^{-\beta z} \cos (\beta z)}\right) .
$$

With the velocity profile given, the viscous forces exerted on the hair are described in (Stokes, 1851), under the assumption of small angular displacements and low Reynolds and Strouhal numbers (Humphrey et al., 1993). Following the analysis of Shimozawa et al. (1998), the torque $T_{\mathrm{f}}$, due to the relative air-movement, acting upon the hair can be expressed as

$$
T_{\mathrm{f}}=\sqrt{A^{2}+B^{2}}
$$

where $A$ and $B$ are torque contributions given by

$$
A=\int_{0}^{L}\left|Z_{\mathrm{S}}\right| V_{z} z \cos \left(\zeta_{z}+\eta_{\mathrm{S}}\right) \mathrm{d} z, \quad B=\int_{0}^{L}\left|Z_{\mathrm{S}}\right| V_{z} z \sin \left(\zeta_{z}+\eta_{\mathrm{S}}\right) \mathrm{d} z
$$

In both $A$ and $B$, the parameter $Z_{\mathrm{S}}$ expresses the relation between the oscillating air-flow $V$ and the force per unit length $F$ :

$$
Z_{\mathrm{S}}=\frac{F}{V}=4 \pi \mu G+j\left(\frac{\pi \rho d^{2}}{4} \omega-\frac{\pi^{2} \mu G}{g}\right) .
$$

Here, $G, g$ and $s$ are dimensionless parameters:

$$
G=\frac{-g}{g^{2}+(\pi / 4)^{2}}, \quad g=\gamma+\ln (s), \quad s=\frac{d}{4} \sqrt{\frac{\omega}{\nu}} .
$$

\section{References}

Humphrey, J., Devarakonda, R., Iglesias, I., and Barth, F. (1993). Dynamics of arthropod filiform hairs. I. Mathematical modeling of the hair and air motions. Phil. Trans.: Bio. Sc., 340:423-444.

Panton, R. L. (1996). Incompressible flow. Wiley.

Shimozawa, T., Kumagai, T., and Baba, Y. (1998). Structural scaling and functional design of the cercal wind-receptor hairs of cricket. J. Comp. Physiol. A, 183:171186.

Stokes, G. G. (1851). On the effect of the internal friction of fluids on the motion of pendulums. Trans. Cambr. Phil. Soc., 9:1-141. 


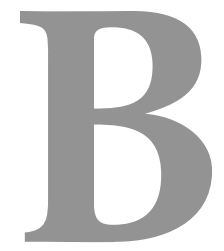

\section{Loudspeaker transduction model}

To determine the frequency range of the loudspeaker as a source for oscillatory flow, we use an energy based derivation (Fluitman, 1986) to describe the behaviour of the loudspeaker (figure B.1) (Dickason, 2006). We consider the loudspeaker as a two-port transducer with free energy $E$ : one mechanical port (moving cone) with external force $F_{\text {ext }}$ and displacement $x$ and one magnetic port (coil) with current $i$ and coupled magnetic flux $\lambda$ (Olson, 1943). Changes in the free energy $E$ occur through the ports:

$$
\mathrm{d} E=F_{\text {ext }} \mathrm{d} x+i \mathrm{~d} \lambda .
$$

The external force $F_{\text {ext }}$ is given by the spring force $F_{\mathrm{s}}$, which is related to $x$ by a spring constant $K$. The relation between $\lambda$ and $i$ are given by a self-inductance $L$, the displacement $x$ and the magnetic remanence $B_{\mathrm{r}}$ :

$$
F_{\mathrm{s}}=K x, \quad \lambda=L i+x B_{\mathrm{r}} .
$$

The total energy $E$ becomes:

$$
E(\lambda, x)=\frac{1}{2} K x^{2}+\frac{1}{2} \frac{\left(\lambda-x B_{\mathrm{r}}\right)^{2}}{L} .
$$




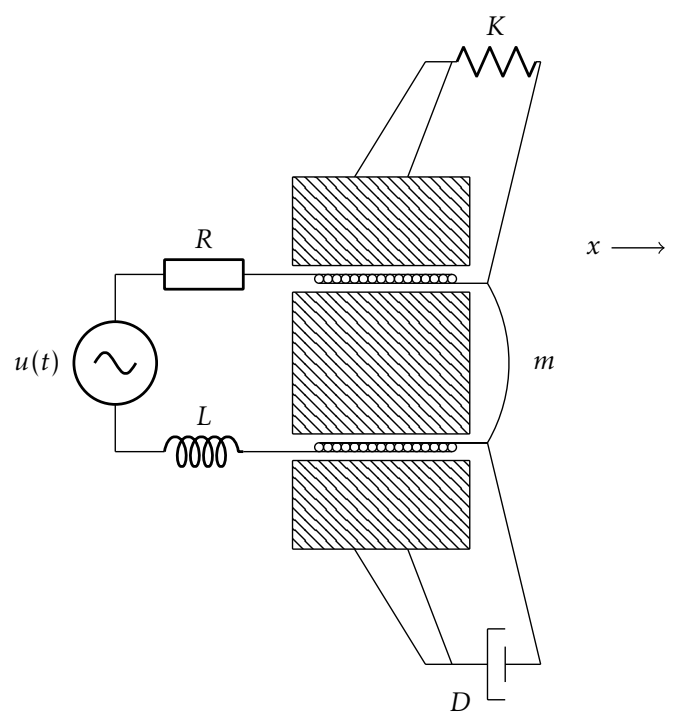

Figure B.1: Schematic view of a loudspeaker.

Differentiating this equation twice with respect to the extensive variables $x$ and $\lambda$ leads to the following matrix:

$$
\left[\begin{array}{c}
\mathrm{d} F \\
\mathrm{~d} i
\end{array}\right]=\left[\begin{array}{cc}
\frac{B_{\mathrm{r}}^{2}}{L}+K & -\frac{B_{\mathrm{r}}}{L} \\
-\frac{B_{\mathrm{r}}}{L} & \frac{1}{L}
\end{array}\right]\left[\begin{array}{l}
\mathrm{d} x \\
\mathrm{~d} \lambda
\end{array}\right] .
$$

Now, we express the force $F$ as:

$$
F=\int\left[\left(\frac{B_{\mathrm{r}}^{2}}{L}+K\right) \frac{\mathrm{d} x}{\mathrm{~d} t}-\frac{B_{\mathrm{r}}}{L} \frac{\mathrm{d} \lambda}{\mathrm{d} t}\right] \mathrm{d} t
$$

and the current $i$ as:

$$
i=\int\left[-\frac{B_{\mathrm{r}}}{L} \frac{\mathrm{d} x}{\mathrm{~d} t}+\frac{1}{L} \frac{\mathrm{d} \lambda}{\mathrm{d} t}\right] \mathrm{d} t .
$$

Here, the derivative of the cone position $x$ is its velocity $v$ and the derivative of the magnetic flux $\lambda$ is the voltage $u$. Assuming the signals to be harmonic and using complex notation with complex amplitudes $\mathbf{v}_{\mathbf{0}}$ and $\mathbf{u}_{\mathbf{0}}$ :

$$
\frac{\mathrm{d} x}{\mathrm{~d} t}=v=\operatorname{ke}\left(v_{0} e^{j \omega t}\right), \quad \frac{\mathrm{d} \lambda}{\mathrm{d} t}=u=\operatorname{kv}\left(\boldsymbol{u}_{0} e^{j \omega t}\right) .
$$

Applying these complex expressions, integrating them over time $t$ and rewriting them, this leads to the following matrix: 


$$
\left[\begin{array}{l}
\boldsymbol{F}_{\mathbf{0}} \\
\boldsymbol{u}_{\mathbf{0}}
\end{array}\right]=\left[\begin{array}{ll}
\frac{K}{j \omega} & -B_{\mathrm{r}} \\
B_{\mathrm{r}} & j \omega L
\end{array}\right]\left[\begin{array}{l}
\boldsymbol{v}_{\mathbf{0}} \\
\boldsymbol{i}_{\mathbf{0}}
\end{array}\right]
$$

The external force $F_{\text {ext }}$ acts on the mass of the cone $m$, which is damped by viscous damping with coefficient $D$ :

$$
m \frac{\mathrm{d} v}{\mathrm{~d} t}=-F_{e x t}-D v .
$$

Since the external force $F_{\text {ext }}$ is directly related to the force amplitude $\boldsymbol{F}_{\mathbf{0}}$ :

$$
\boldsymbol{F}_{\mathbf{0}}=-(j \omega m+D) \boldsymbol{v}_{\mathbf{0}}
$$

With the combination of the previous expressions, we can calculate the impedance of the loudspeaker coil $Z_{L}$ :

$$
Z_{\mathrm{L}}=j \omega L \frac{-m \omega^{2}+j \omega D+\left(K+\frac{B_{\mathrm{r}}^{2}}{L}\right)}{-m \omega^{2}+j \omega D+K} .
$$

The total impedance $Z_{\mathrm{t}}$ is the sum of the resistance $R$ of the coil itself and its impedance $Z_{\mathrm{L}}$ :

$$
Z_{\mathrm{t}}=R+Z_{\mathrm{L}}
$$

When a voltage is applied to the loudspeaker, the velocity amplitude $\boldsymbol{v}_{\mathbf{0}}$ can be calculated from it:

$$
\boldsymbol{v}_{\mathbf{0}}=-\frac{j \omega B_{\mathrm{r}}}{m \omega^{2}-j \omega D-K} \boldsymbol{i}_{\mathbf{0}} .
$$

The current $i_{0}$ is calculated from the applied voltage amplitude $u_{0}$ and the impedance $Z_{\mathrm{t}}$ :

$$
i_{0}=\frac{u_{0}}{Z_{\mathrm{t}}}
$$

When we apply this model to our loudspeaker and compare it to measurements performed using a Polytec Laser Scanning Vibrometer (Polytec, 2005), only a fit factor of 1.15 was required to fit the data on the measurement data, which is shown in figure B.2. 

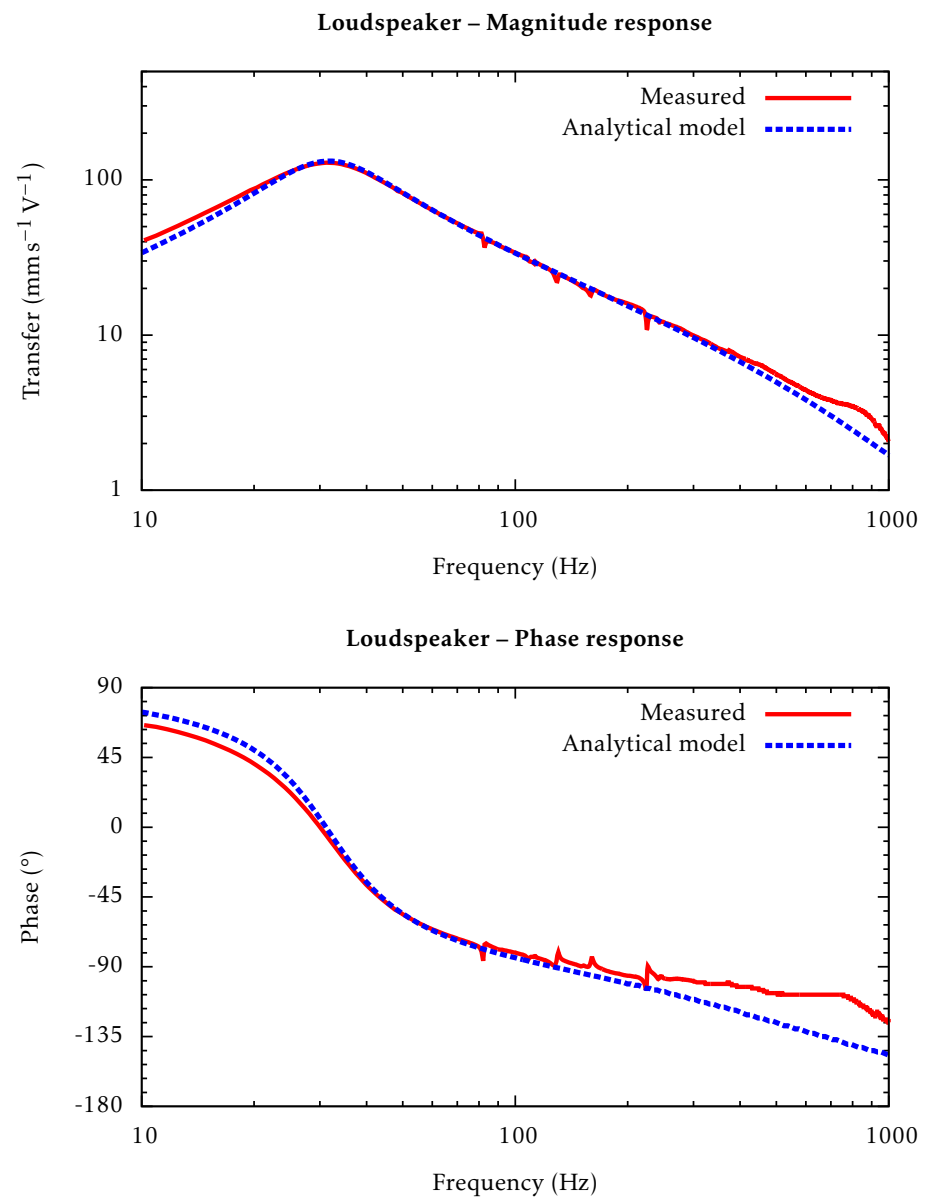

Figure B.2: Model and results for the used loudspeaker (Visaton WS 17 E).

\section{References}

Dickason, V. (2006). Loudspeaker design cookbook. Audio Amateur Press, Peterborough, $7^{\text {th }}$ edition.

Fluitman, J. H. J. (1986). Transduktietechniek, vol. 070.749. University of Twente.

Olson, H. F. (1943). Dynamical analogies. D. van Nostrand Company, New York.

Polytec (2005). MSA-400 Micro System Analyzer. Polytec, Waldbronn, Germany. 


\section{C \\ Pressure responsivity}

To electrically read-out our hair flow sensors, the sensor's capacitance is measured (Dagamseh et al., 2013). This principle is schematically shown in figure C.1, where $g$ is the distance between the membrane and the substrate and $L$ is the length of the membrane. In the presence of airflow, the hair tilts and consequently the capacitors change equally but oppositely. On application of differential capacitive read-out, the difference in capacitance $\Delta C$ between both halves of the membrane is effectively measured, so that for the voltage $U$ one can write:

$$
U(v, p) \propto \Delta C(v, p) \propto \int_{-w / 2}^{w / 2} \int_{0}^{L / 2} \frac{1}{g+\xi+\theta x} \mathrm{~d} x \mathrm{~d} w-\int_{-w / 2}^{w / 2} \int_{0}^{L / 2} \frac{1}{g+\xi-\theta x} \mathrm{~d} x \mathrm{~d} w,
$$

where $\theta$ is the membrane rotation, $w$ is the width of the membrane and $\xi$ is the pressure difference induced movement of the membrane normal to the substrate. Notice that responsivity to pressure can be expected due to the significant amount of squeeze film damping in the gap between the capacitor plates (Bao and Yang, 2007). Combining both terms from (C.1) gives:

$$
U(v, p) \propto w \int_{0}^{L / 2} \frac{-2 \theta x}{(g+\xi)^{2}-(\theta x)^{2}} \mathrm{~d} x \approx-\frac{w L^{2}}{2}\left[\frac{\theta}{g^{2}+2 g \xi}\right],
$$




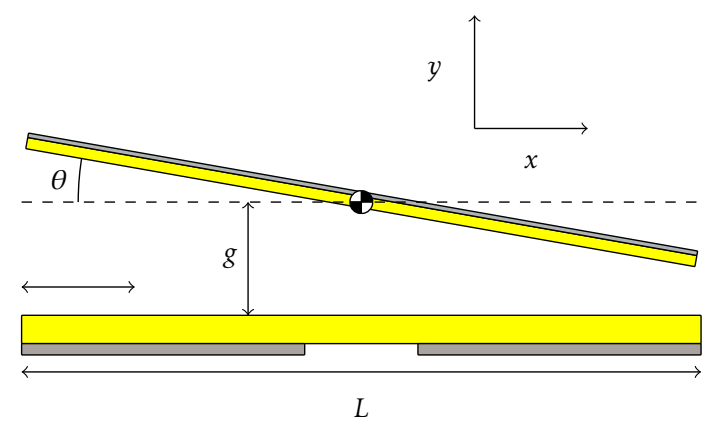

Figure C.1: Geometry of the angle-dependent rectangular capacitor.

where the last approximation is allowed due to the very small rotation angles normally encountered $(\theta L / 2 \ll g)$ and where $\xi \ll g$ has been assumed. The rotation angle may consist of a small constant tilt $\theta_{0}$ and a time-dependent rotation proportional to the flow velocity $v$ whereas the vertical displacement will be proportional to the pressure difference $p$ :

$$
\theta=\theta_{0}+H_{\mathrm{v}}(\omega) v, \quad \xi=H_{\mathrm{p}}(\omega) p,
$$

where $H_{\mathrm{v}}(\omega)$ and $H_{\mathrm{p}}(\omega)$ are the frequency-dependent mechanical transfer functions for flow and pressure respectively. Inserting these relations into (C.2) gives:

$$
U(v, p, \omega) \propto-\frac{w L^{2}}{2 g}\left[\frac{\theta_{0}+H_{\mathrm{v}}(\omega) v}{g+2 H_{\mathrm{p}}(\omega) p}\right] .
$$

Approximating the denominator by its first order Taylor expansion we find:

$$
\begin{aligned}
U(v, p, \omega) & \approx \frac{w L}{2 g^{2}}\left[\theta_{0}+H_{\mathrm{v}}(\omega) v\right]\left[1-\frac{2 H_{\mathrm{p}}(\omega) p}{g}\right] \\
& =\frac{w L}{2 g^{2}}\left[\theta_{0}+H_{\mathrm{v}}(\omega) v-\theta_{0} \frac{2 H_{\mathrm{p}}(\omega) p}{g}-\frac{2 H_{\mathrm{p}}(\omega) p H_{\mathrm{v}}(\omega) v}{g}\right] .
\end{aligned}
$$

Finally, for harmonic flow $v=v_{0} e^{j \omega t}$ and pressure $p=Z(\omega) v_{0} e^{j \omega t}$, and retaining only the terms at $\omega$ we find:

$$
\begin{aligned}
U\left(v_{0}, \omega\right) & \approx \frac{w L^{2}}{2 g^{2}}\left[H_{\mathrm{v}}(\omega)-\theta_{0} Z(\omega) \frac{2 H_{\mathrm{p}}(\omega)}{g}\right] v_{0} e^{j \omega t}, \\
& =\frac{w L^{2}}{2 g^{2}}\left[H_{\mathrm{v}}(\omega)-\theta_{0}|Z(\omega)| \frac{2 H_{\mathrm{p}}(\omega)}{g} e^{j \phi}\right] v_{0} e^{j \omega t},
\end{aligned}
$$


where $\phi$ is the phase shift that depends on the acoustic impedance $Z(\omega)$. From (C.6) it is clear that the sensor response has both particle velocity and pressure contributions and the importance of both critically depends on the acoustic impedance, both its magnitude and phase, as well as on an existing asymmetry of the membrane, i.e. the offset angle $\theta_{0}$. Especially in a standing wave tube, the acoustic impedance $|Z(\omega)|$ can be very large.

\section{References}

Bao, M. and Yang, H. (2007). Squeeze film air damping in MEMS. Sens. Act. A: Phys., 136:3-27.

Dagamseh, A. M. K., Bruinink, C. M., Wiegerink, R. J., Lammerink, T. S. J., Droogendijk, H., and Krijnen, G. J. M. (2013). Interfacing of differentialcapacitive biomimetic hair flow-sensors for optimal sensitivity. J. Micromech. Microeng., 23:035010. 


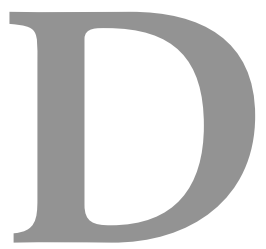

\section{Capacitive read-out}

For capacitive read-out of the hair rotational angle, a charge amplifier based circuit is use based on the read-out method described by Haneveld et al. (2010). As shown in figure D.1, the sensor's response is measured differentially by using two counterphase signals at a frequency of $1 \mathrm{MHz}$ with amplitude $V_{s}$. The capacitive structures $C_{1}$ and $C_{2}$ of the sensor are electrically separated by a decoupling capacitor $C_{c}$, in order to control both the biasing of the sensor (for implementation of electrostatic spring softening) and bias control of the read-out electronics.

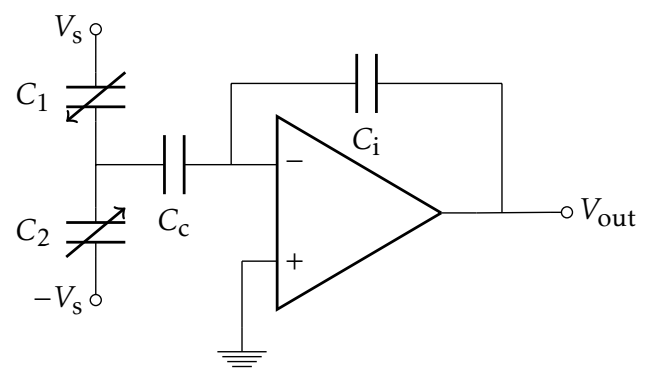

Figure D.1: Differential capacitive measurement of the hair flow sensor.

In contrast to (Haneveld et al., 2010), a JFET buffer is implemented in the 


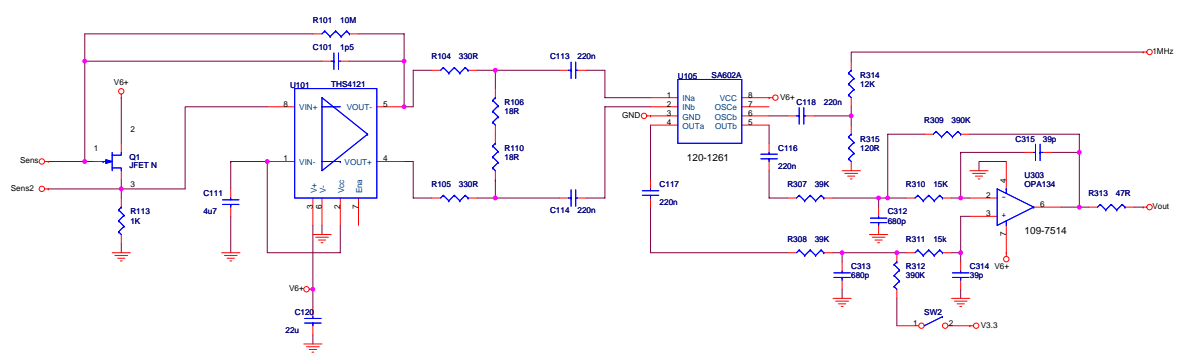

(a) Charge amplifier circuit.

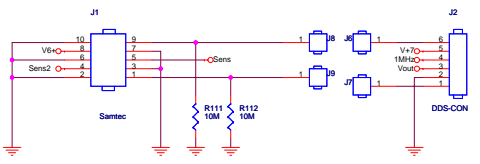

(b) SMT connectors.

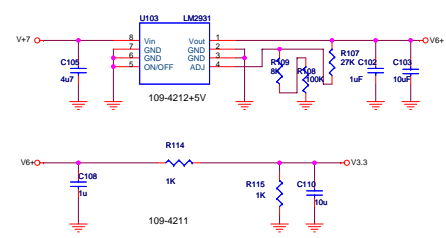

(c) Power supply.

Figure D.2: Electronic diagram of the capacitive read-out electronics.

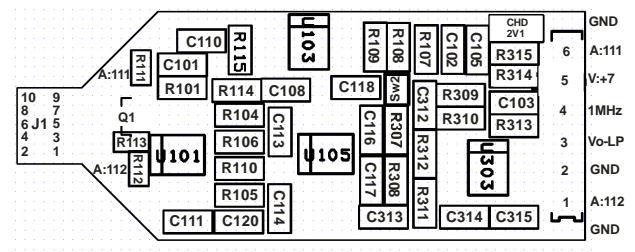

Figure D.3: Top side lay-out of the capacitive read-out electronics.

electronic circuitry used for our flow measurements. Generally, the use of a JFET buffer allows for low noise measurements in MEMS sensors (Bernstein et al., 1999). The full electronic diagram is shown in figure D.2, with from left to right: the JFET buffer, the charge amplifier stage based on a THS 4121 chip, buffer circuitry with the demodulator (SA602A), and an active band-pass filter output stage using a OPA134 chip. This last stage is used for noise-reduction and differential amplification of the small output signals generated by the charge amplifier circuitry. The band-pass characteristics of the filter are shown in figure D.4.

To get some insight in the noise generated in the electronics, small-signal noise analysis is performed using LTSpice. However, this approach is only valid for estimating the noise generated in the charge amplifier stage and the band-pass filter stage. To simulate noise in e.g. mixers, oscillator and sampled-data systems, small-signal noise analysis does not suffice and real-time noise analysis is required (Phillips and Klundert, 2000), for which the appropriate software is not available 


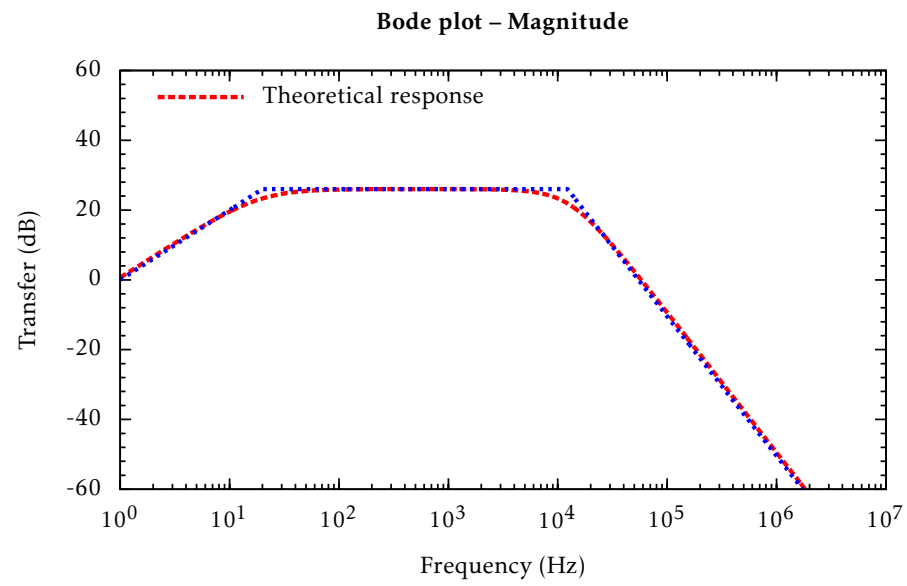

Figure D.4: Transfer of the band-pass filter stage with the $-3 \mathrm{~dB}$ points located at approximately $20 \mathrm{~Hz}$ and $10 \mathrm{kHz}$.

to investigate these contributions. The simulation results for the charge amplifier stage by also taking into account the on-chip capacitances and resistances of a single hair flow sensor (Dagamseh et al., 2013) are shown in figure D.5. Also, the noise generated by the mixer (without carrier signals) and the band-pass filter stage is shown.

We observe that the band-pass filter stage has a noise density of approximately $0.45 \mu \mathrm{V} \sqrt{\mathrm{Hz}}^{-1}$, wherein the band-pass filter characteristics with the specified cutoff frequencies from figure D.4 can be clearly recognized. Although the simulated noise density in the stage before the mixer is significantly lower and shows a low-pass filter characteristic with its cut-off around $3 \mathrm{MHz}$, this noise spectrum will be affected by the mixer and band-pass filter characteristics.

The used mixer (SA602A) has a specified gain of $18 \mathrm{~dB}$ and a noise figure of less than $5 \mathrm{~dB}$ at a frequency of $45 \mathrm{MHz}$. Approximately, the noise at the input of the mixer with a density of about $0.02 \mu \mathrm{V} \sqrt{\mathrm{Hz}}^{-1}$ will be upconverted by the mixer with a frequency shift of $1 \mathrm{MHz}$ and is amplified about eight times by the mixer. Further, the band-pass filter stage also amplifies the input signals about ten times. As a consequence, the noise generated in the charge amplifier stage will be at the output of the same order as the noise generated in the band-pass filter stage, as shown in figure D.5. By taking the integral of the quadratically summed noise contributions (dash-dotted blue and dotted green lines), a rough estimate can be made for the output rms-voltage of the read-out electronics, for which an output rms-voltage is calculated of $0.21 \mathrm{mV}$. 


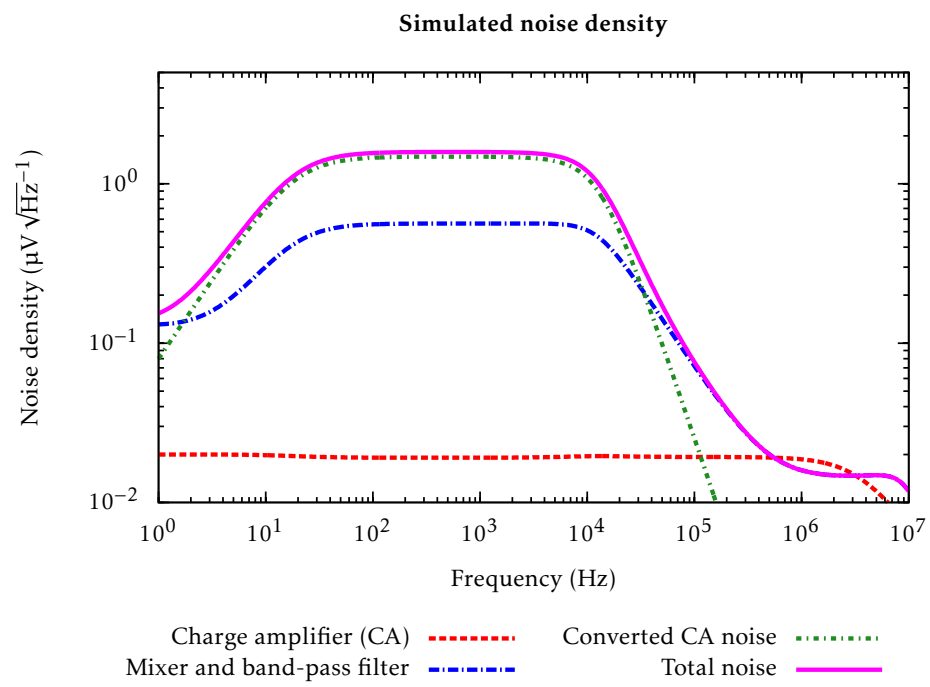

Figure D.5: Simulated noise density using small-signal noise analysis in LTSpice.

\section{References}

Bernstein, J., Miller, R., Kelley, W., and Ward, P. (1999). Low-noise MEMS vibration sensor for geophysical applications. J. Microelectromech. S., 8(4):433-438.

Dagamseh, A. M. K., Bruinink, C. M., Wiegerink, R. J., Lammerink, T. S. J., Droogendijk, H., and Krijnen, G. J. M. (2013). Interfacing of differentialcapacitive biomimetic hair flow-sensors for optimal sensitivity. J. Micromech. Microeng., 23:035010.

Haneveld, J., Lammerink, T. S. J., de Boer, M. J., Sanders, R. G. P., Mehendale, A., Lötters, J. C., Dijkstra, M., and Wiegerink, R. J. (2010). Modeling, design, fabrication and characterization of a micro Coriolis mass flow sensor. J. Micromech. Microeng., 20:125001.

Phillips, J. and Klundert, K. (2000). An introduction to cyclostationary noise. The Designer's Guide Community. 


\section{$\mathrm{E}$ \\ Process flow for hair sensors}

\section{E.1 Introduction}

In this process flow the fabrication process of artificial bio-inspired hair sensors is given, based on the process flow for MEMS single-hair flow sensors described in chapter 1. A new feature in this generation hair sensors is the inclusion of a second (thin) SiRN layer, for adding thin and long springs for implementation of either a non-linear or a reduced torsional stiffness. Additionally, thin $\mathrm{SiO}_{2}$ layer are realized for extra protection of the nitride layers during Sacrificial Layer Etching (SLE) by $\mathrm{XeF}_{2}$. Also, the gap is increased to $1.5 \mu \mathrm{m}$ to allow for designs which need mechanical non-linearity, a larger rotational angle for operation or reduced squeezed film damping.

\section{E.2 Specific design properties}

\section{E.2.1 Design rules}

The wafer is divided in square sections of $8 \mathrm{~mm} \times 8 \mathrm{~mm}$, each containing a specific sensor design. For the basic hair sensor design, a set of design rules applies, which are shown in table E.1. 
Table E.1: Design rules for MEMS hair flow sensors.

\begin{tabular}{ll}
\hline Quantity & Value \\
\hline Width of beams & $5 \mu \mathrm{m}$ \\
Length of beams & $75 \mu \mathrm{m}$ \\
Hair length & $800 \mu \mathrm{m}$ \\
Wafer type & SOI (silicon-on-insulator) \\
Chip size & $8 \mathrm{~mm} \times 8 \mathrm{~mm}$ \\
Wafer orientation & $<100>$ \\
Minimal design width & $2.5 \mu \mathrm{m}$ \\
Sacrificial layer (gap) thickness & $>1.5 \mu \mathrm{m}$ \\
\hline
\end{tabular}

\section{E.2.2 Masks}

The first mask TRENCH contains the pattern of the electrical connections to the sensors consisting of the high frequency modulation signal for capacitive read-out capability. These connections can also be used to apply an arbitrary voltage for Electrostatic Spring Softening (ESS). The mask SACRI defines the openings for sacrificial layer etching (SLE), to prevent (undesired) etching of the sacrificial layer. Using the mask $M E M B R 1$ the membranes, springs and other typical sensors aspects are realized. The mask $M E M B R 2$ allows for realizing the thin beams for adding stiffness functionality to the hair flow sensors. The mask ELECT defines the pattern of the aluminium electrodes, which are used for capacitive readout (containing the angle information) and Electrostatic Spring Softening (ESS). The SU-8 hairs are created using the masks HAIR1 and HAIR2. In order to create uniform, long and thick hairs, the mask HAIR 1 can also be used twice. An overview of the masks is shown in table E.2.

\section{E.2.3 Flat SiRN membrane for springs and membranes}

To realize a flat SiRN membrane for the springs and the membranes of the artificial hair sensors, a special step using thermal oxidation of polysilicon should be used. To explain this, have a look at figure E.1. When a gap of $600 \mathrm{~nm}$ is desired, the thickness of the polysilicon should be $600 \mathrm{~nm}$ (for a gap of $1 \mu \mathrm{m}$ a thickness of $1 \mu \mathrm{m}$ is required). However, due to the trenches for realizing the electrical connections for supplying the modulation signals, the layer of polysilicon will not be entirely 
Table E.2: Mask overview for fabrication of MEMS hair flow sensors.

\begin{tabular}{lll}
\hline \# & Name & Description \\
\hline $\mathbf{1}$ & TRENCH & Patterning of the device layer and alignment marks \\
$\mathbf{2}$ & SACRI & Defining the etch regions for SLE \\
$\mathbf{3}$ & MEMBR1 & The springs and membranes of the sensors \\
$\mathbf{4}$ & MEMBR2 & The thin support beams of the sensors \\
$\mathbf{5}$ & ELECT & Aluminium electrodes \\
$\mathbf{6}$ & HAIR1 & Lower part of the SU-8 hair \\
$\mathbf{7}$ & HAIR2 & Upper part of the SU-8 hair \\
\hline
\end{tabular}

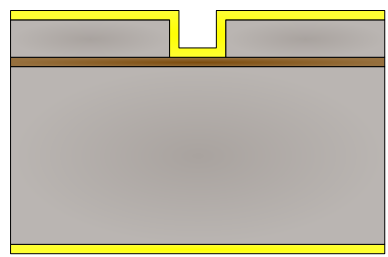

(a) Deposition of $\mathrm{Si}_{3} \mathrm{~N}_{4}$.

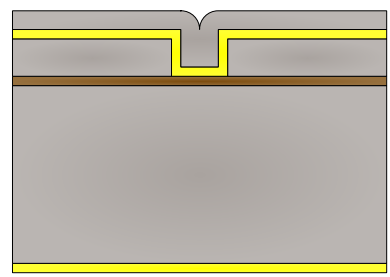

(b) Deposition of poly-Si $(\leq 1000 \mathrm{~nm})$.

Figure E.1: Deposition of poly-Si without thermal oxidation step.

flat (see figure E.1b).

To overcome this, the layer thickness of poly-silicon is increased to a minimum thickness in order to completely fill the trench (which is about $2.5 \mu \mathrm{m}$ wide). Doing so, the filling effect of the trenches becomes negligible, leading to an almost flat surface of the poly-Si. To achieve the desired gap of $600 \mathrm{~nm}$, thermal oxidation is used to convert a part of the poly-Si into $\mathrm{SiO}_{2}$. The resulting $\mathrm{SiO}_{2}$ layer is removed using an etchant, leading to a sacrificial layer with the desired thickness, which is all given in figure E.2.

\section{E.3 Experimental details}

This section is taken from unpublished documentation by Christiaan Bruinink (2009).

\section{E.3.1 Trench filling and thinning by oxidation of poly-silicon}

Several test experiments have been done to determine the oxidation rate of polysilicon under dry and wet oxidation conditions (see figure E.3).

In order to completely fill the $2-2.5 \mu \mathrm{m}$ wide trench (and finally obtain a 


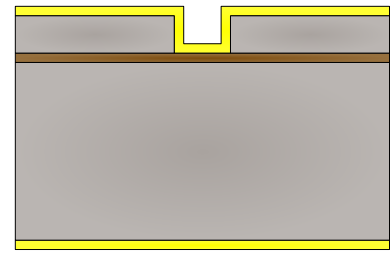

(a) Deposition of $\mathrm{Si}_{3} \mathrm{~N}_{4}$.

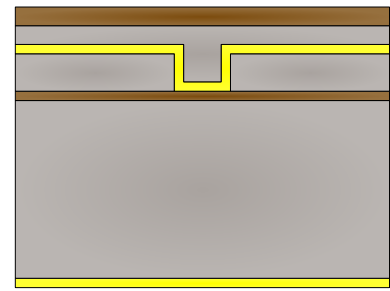

(c) Thermal oxidation of poly-Si $(\leq 800 \mathrm{~nm})$.

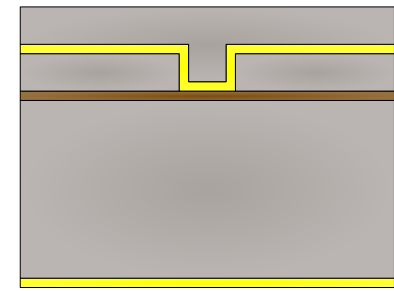

(b) Deposition of poly-Si $(1400 \mathrm{~nm})$.

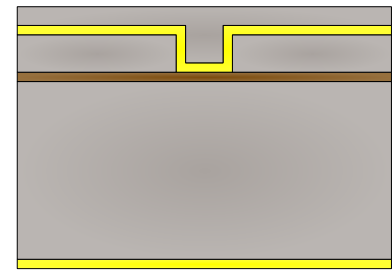

(d) Removal of $\mathrm{SiO}_{2}$.

Figure E.2: Deposition of poly-Si with thermal oxidation step.

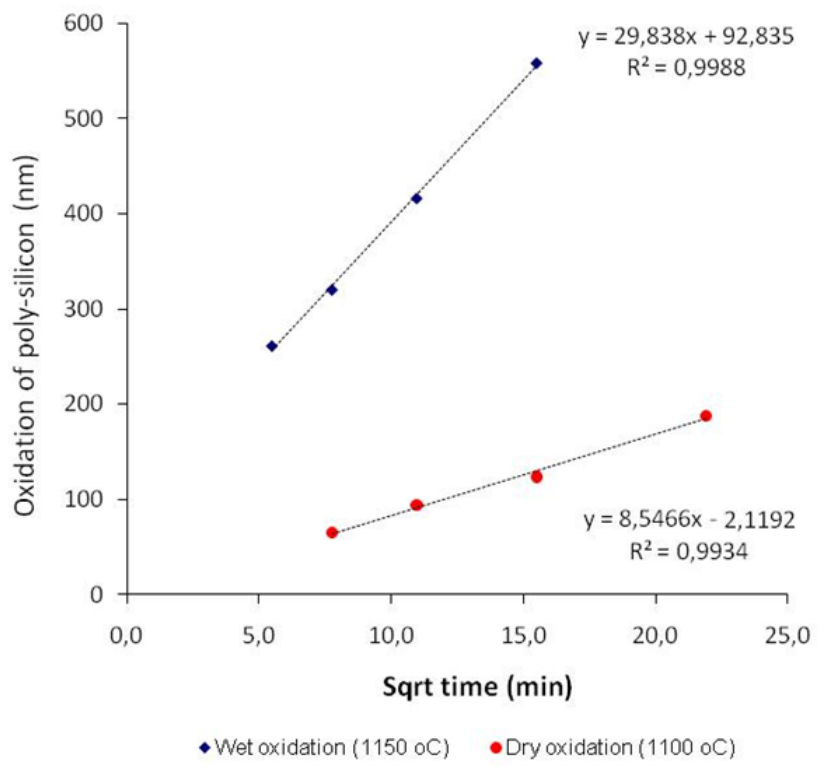

Figure E.3: Graph illustrating the oxidation rate of poly-silicon under two different oxidation conditions. 


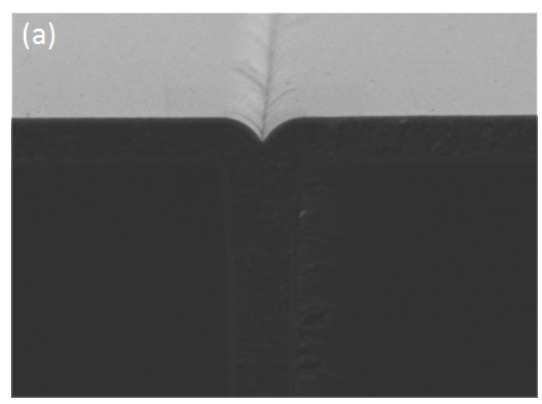

(a) Trench after filling with $200 \mathrm{~nm}$ $\mathrm{Si}_{3} \mathrm{~N}_{4}$ and $1400 \mathrm{~nm}$ poly-silicon.

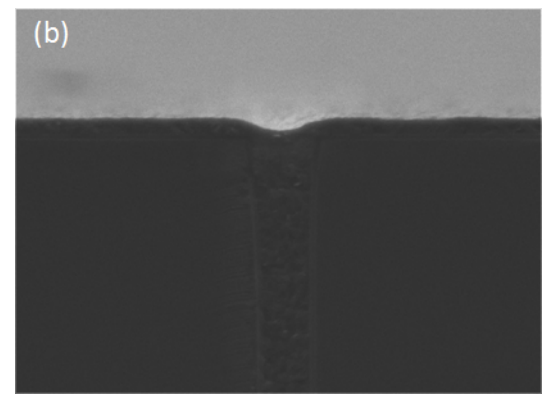

(b) Trench after two consecutive stages of wet oxidation $\left(1150^{\circ} \mathrm{C}, 120 \mathrm{~min}\right)$ and wet-chemical etching in BHF (18 min).

Figure E.4: SEM images of trench filling.
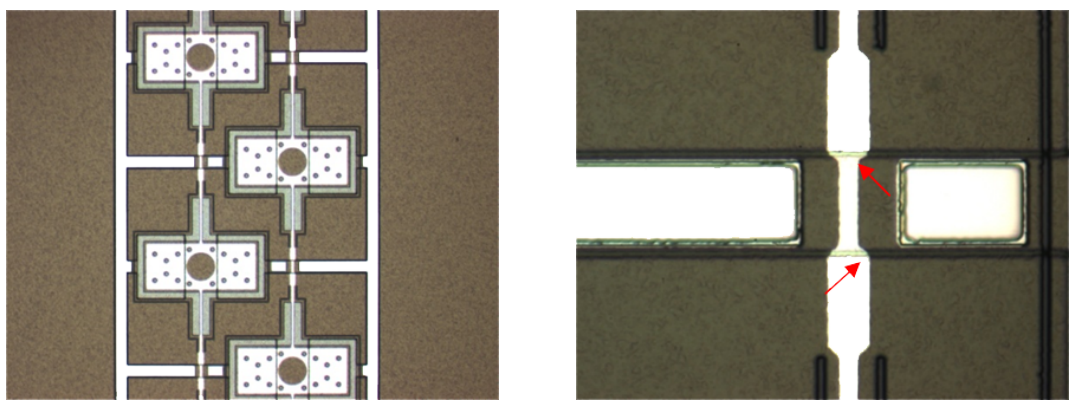

Figure E.5: Optical microscope images of the device wafer at the stage after sputtering and patterning of aluminium. The red arrows in the close-up image (on the right) illustrate aluminium wires running over the transition points.

smooth transition across the isolation trench, the decision has been made to deposite (besides the $200 \mathrm{~nm}$ of $\mathrm{Si}_{3} \mathrm{~N}_{4}$ ) $1400 \mathrm{~nm}$ poly-silicon by LPCVD. Figure E.4a illustrates the sharp transition at the location of the trench after LPCVD.

A deposition of $1400 \mathrm{~nm}$ poly-silicon requires two consecutive wet-oxidation runs of two hours (see figure E.3) to obtain a sacrificial poly-silicon layer of $600 \mathrm{~nm}$ in thickness. As is shown in figure E.4b, the thinning of poly-silicon by wet oxidation and wet-chemical etching in BHF was successful in two aspects: (1) the thinning of the poly-silicon was very accurate (due to differences in starting poly-silicon layer, the final thickness of the poly-silicon layer on different device wafers were varying from $570 \mathrm{~nm}$ to $610 \mathrm{~nm}$, and (2) the transition was gradual for patterning aluminum wires in a reliable manner (see figure E.5). One point of attention is the increase in the surface roughness by about $20 \mathrm{~nm}$. 


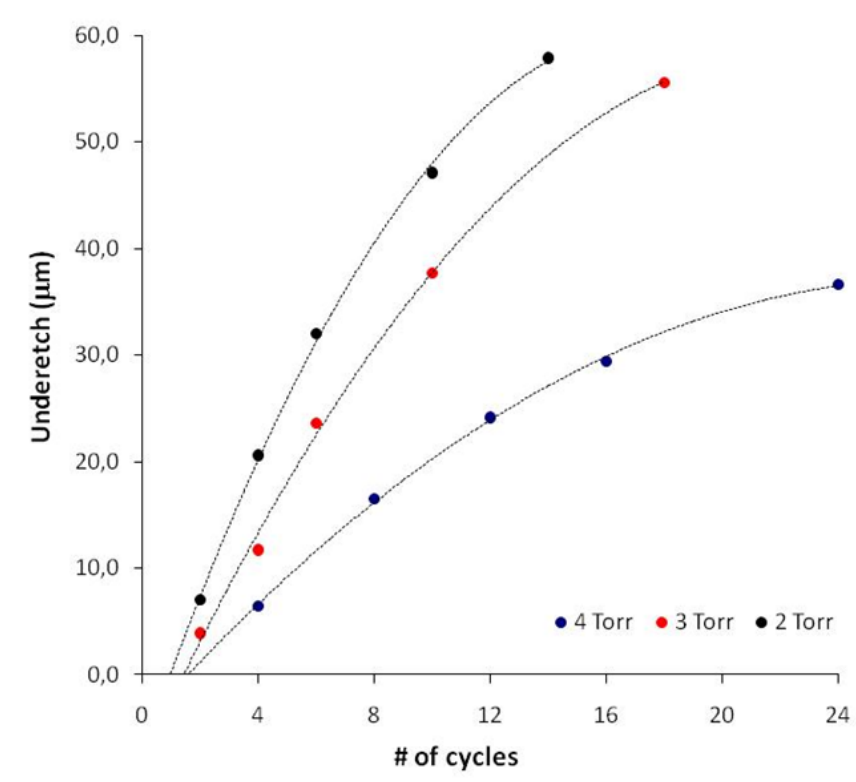

Figure E.6: Graph illustrating the underetch as a function of the number of etch cycles and the pressure.

\section{E.3.2 Sacrificial layer etching by $\mathrm{XeF}_{2}$}

Several test experiments have been done to determine the etch rate of poly-silicon under different conditions (see figure E.6). All these experiments have a constant cycle time of $30 \mathrm{~s}$ and measurements are done on circular features with a lateral dimension of $10 \mu \mathrm{m}$ at five different locations on a 4-inch wafer (centre, top, bottom, left and right of the wafer).

Of importance is the pre-treatment of the wafer ( $1 \mathrm{~min}$ in BHF or $5 \mathrm{~min} 1 \%$ $\mathrm{HF}+1$ min dehydration bake at $120^{\circ} \mathrm{C}$ ). The etching will not initiate without a proper removal of the (native) oxide layer. The $\mathrm{XeF}_{2}$ is so selective, that without this (native) oxide removal step the $\mathrm{XeF}_{2}$ is not able to etch through this protective layer. For our device wafers, wet-chemical methods to remove this oxide are not compatible, therefore a short etch in the Oxford Plasmalab $100(10 \mathrm{mtorr}, 120 \mathrm{sccm}$ $\mathrm{SF}_{6}, 600 \mathrm{~W}$ ICP, $\left.2.5 \mathrm{~W} \mathrm{CCP}, 20^{\circ} \mathrm{C}, 1 \mathrm{~min}\right)$.

As is shown in table E.3, it takes about 1-2cycles to initiate the underetching of poly-silicon and depending on the conditions the underetch rate varies from about 2-6 $\mu \mathrm{m} \mathrm{min}^{-1}$. The decision has been made to etch the device wafers using the following conditions: 3 torr, 30 s cycle time, 10 cycles (see figure E.7). 


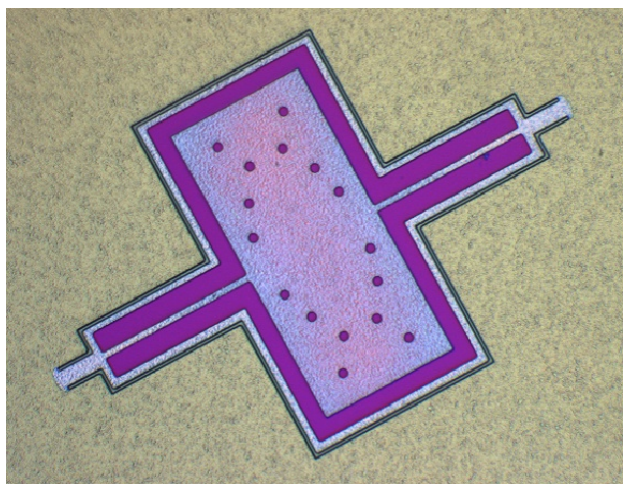

Figure E.7: Optical microscope image of a device test structure (without aluminium electrodes and SU-8 hair) after etching the sacrificial poly-silicon with $\mathrm{XeF}_{2}$ (3torr, $30 \mathrm{~s}$ cycle time, 10 cycles).

Table E.3: Values for $\mathrm{XeF}_{2}$ sacrificial layer etching.

\begin{tabular}{cccc}
\hline $\begin{array}{c}\text { Pressure } \\
\text { (Torr) }\end{array}$ & $\begin{array}{c}\text { Initiation time } \\
\text { (cycles) }\end{array}$ & $\begin{array}{c}\text { Etch rate } \\
(\boldsymbol{\mu} \mathrm{m} / \text { cycle })\end{array}$ & $\begin{array}{c}\text { Time for completion } \\
\text { (cycles) }\end{array}$ \\
\hline 2 & 1.6 & 2.2 & 21.5 \\
3 & 1.4 & 4.9 & 9.2 \\
4 & 1.0 & 6.2 & 6.8 \\
\hline
\end{tabular}




\section{E.4 Process outline}

Step Process Cross-section after process

I

- Substrate selection

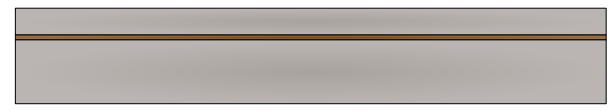

II

- Wafer cleaning

- Patterning of device (Si) layer

- Measurement/inspection

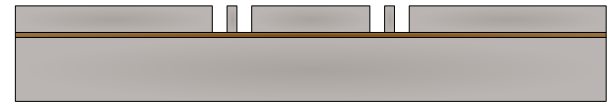

III

- Wafer cleaning

- Deposition of $\mathrm{Si}_{3} \mathrm{~N}_{4}$

- Measurement/inspection

IV

- Wafer cleaning

- Deposition/annealing of poly-Si

- Measurement/inspection

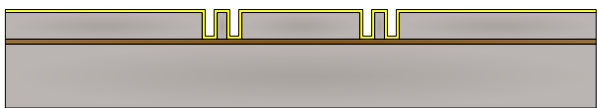

- Thermal wet oxidation

- $\mathrm{SiO}_{2}$ etching for desired thickness

V

- Wafer cleaning

- Patterning poly-Si for trenches

- Measurement/inspection
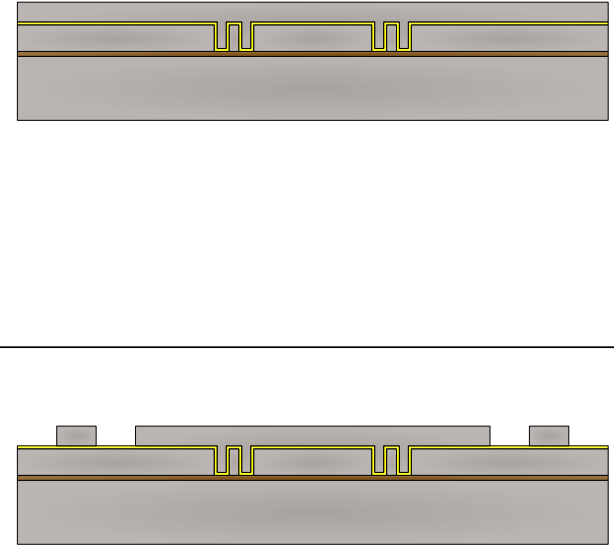


\section{Step Process}

\section{Cross-section after process}

VI

- Wafer cleaning

- Thermal dry oxidation

- Measurement/inspection

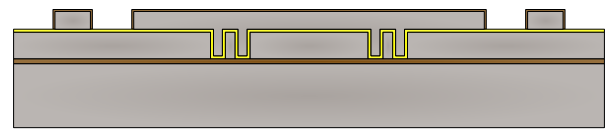

VII

- Wafer cleaning

- Deposition of SiRN for membranes/springs

- Measurement/inspection

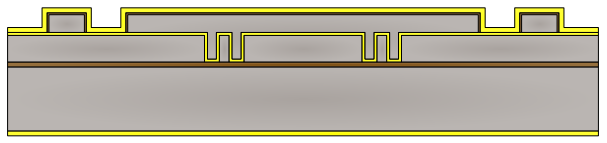

VIII

- Wafer cleaning

- Patterning of SiRN for membranes/springs

- Measurement/inspection

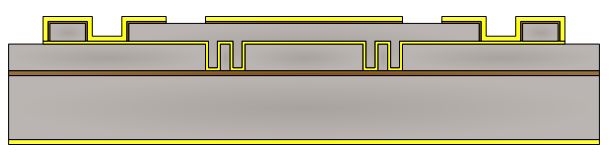

IX

- Wafer cleaning

- Thermal dry oxidation

- Measurement/inspection

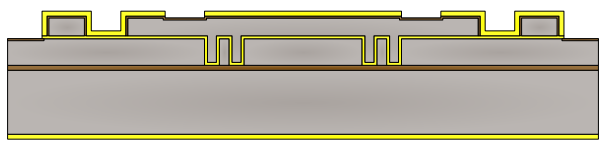

- Wafer cleaning

- Deposition of SiRN for beams

- Measurement/inspection

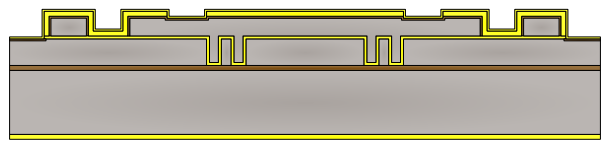

- Wafer cleaning

- Patterning of SiRN for beams

- Measurement/inspection

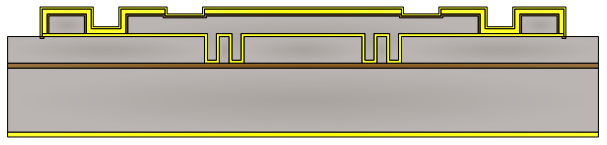




\section{Step Process Cross-section after process}

XII

- Wafer cleaning

- Sputtering and etching of aluminum

- Measurement/inspection

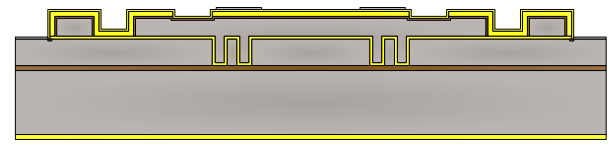

XIII

- Wafer cleaning

- Patterning of SiRN (backside)

- Measurement/inspection

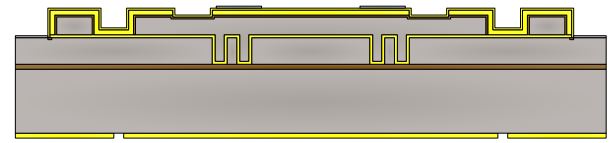

XIV

- Wafer cleaning

- SU-8 lithography (hairs)

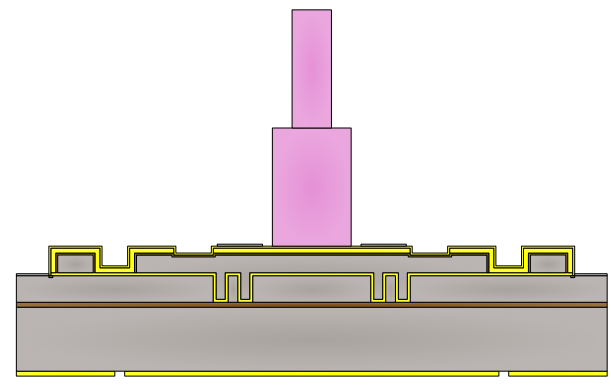

XV

- Sacrificial layer etching

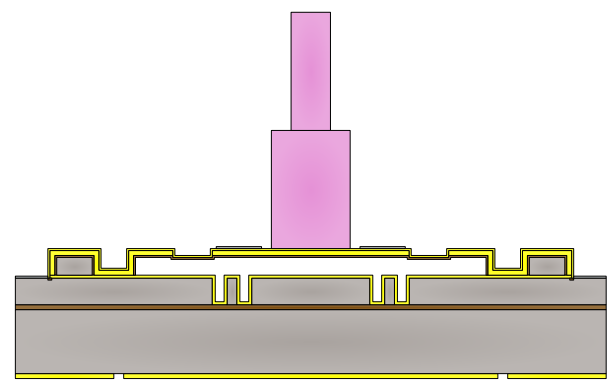




\section{E.5 Process parameters}

\section{E.5.1 Mask inspection}

\begin{tabular}{lll}
\hline Step & Process & \\
\hline $\mathbf{1}$ & $\begin{array}{l}\text { Mask inspection } \\
\text { (\#char001) }\end{array}$ & CR NL / Nikon Microscope \\
& & Use form/logbook \\
\hline
\end{tabular}

\section{E.5.2 Substrate selection}

\begin{tabular}{lll}
\hline Step & Process & \\
\hline 2 & Substrate & Supplier: Ultrasil \\
selection - SOI & Orientation: $<100>$ \\
& Diameter: $100.0 \pm 0.1 \mathrm{~mm}$ \\
& & Device layer: \\
& - Thickness: $25.0 \pm 0.5 \mu \mathrm{m}$ \\
& - Resistivity: $0.01-0.02 \Omega \mathrm{cm}$ \\
& - Dopant: $\mathrm{P} / \mathrm{B}$ \\
& Oxide layer: \\
& - Thickness: $2 \mu \mathrm{m}$ \\
& Handle layer: \\
& - Thickness: $385 \pm 10 \mu \mathrm{m}$ \\
& - Resistivity: $1-20 \Omega \mathrm{cm}$ \\
& \\
\hline
\end{tabular}

\section{E.5.3 Patterning of the device layer - SOI}

\begin{tabular}{lll}
\hline Step & Process & \\
\hline 3 & Standard cleaning $\mathrm{HNO}_{3}$ & $\mathrm{NL}^{2} \mathrm{CR}-\mathrm{WB} 14$ \\
& $\mathrm{HNO}_{3}(99 \%)$ Selectiepur: MERCK \\
& $\mathrm{HNO}_{3}(69 \%)$ VLSI: MERCK \\
& - Beaker 1: fumic $\mathrm{HNO}_{3}(99 \%), 5 \mathrm{~min}$ \\
& - Beaker 2: fumic $\mathrm{HNO}_{3}(99 \%), 5 \mathrm{~min}$ \\
& - Quick Dump Rinse: $<0.1 \mu \mathrm{S}$ \\
& - Beaker 3: boiling $\left(95^{\circ} \mathrm{C}\right) \mathrm{HNO}_{3}(69 \%), 10 \mathrm{~min}$ \\
& - Quick Dump Rinse: $<0.1 \mu \mathrm{S}$ \\
& - Spin drying
\end{tabular}




\begin{tabular}{|c|c|c|}
\hline \multirow[t]{5}{*}{4} & $\begin{array}{l}\text { Standard lithography } \\
\text { Olin 907-17 } \\
(\# \text { lith1002) }\end{array}$ & $\begin{array}{l}\text { NL-CR } \\
\text { Continue immediately with } \\
\text { priming the step! }\end{array}$ \\
\hline & Dehydration bake & $\begin{array}{l}\text { NL-CR / WB } 21-22 \\
\text { - Hotplate: } 120^{\circ} \mathrm{C}: 5 \mathrm{~min}\end{array}$ \\
\hline & Priming (HMDS) & $\begin{array}{l}\text { Spincoater } \\
\text { - Spin program: } 4 \text { (4000 rpm, 30s) }\end{array}$ \\
\hline & Coating (OLIN 907-17) & $\begin{array}{l}\text { Primus Spinner } \\
\text { - Spin program: } 4(4000 \mathrm{rpm}, 30 \mathrm{~s}, 1.7 \mu \mathrm{m}) \\
\text { - Prebake }\left(95^{\circ} \mathrm{C}\right): 90 \mathrm{~s}\end{array}$ \\
\hline & $\begin{array}{l}\text { Alignment \& Exposure } \\
\text { (Olin 907-17) }\end{array}$ & $\begin{array}{l}\text { Electronic Vision Group } 620 \text { Mask Aligner } \\
\text { - Hg-lamp: } 12 \mathrm{~mW} \mathrm{~cm}^{-2} \\
\text { - Exposure time: } 3.8 \mathrm{~s} \\
\text { - Mask: TRENCH }\end{array}$ \\
\hline & Development (Olin 907-17) & $\begin{array}{l}\text { Developer: OPD } 4262 \\
\text { - After exposure bake }\left(120^{\circ} \mathrm{C}\right): 60 \mathrm{~s} \\
\text { - Time: } 30 \mathrm{~s} \text { in beaker } 1 \\
\text { - Time: } 15-30 \mathrm{~s} \text { in beaker } 2 \\
\text { - Quick Dump Rinse: }<0.1 \mu \mathrm{S} \\
\text { - Spin drying }\end{array}$ \\
\hline & Postbake (Olin 907-17) & - Hotplate: $120^{\circ} \mathrm{C}: 0 \mathrm{~min}$ \\
\hline & $\begin{array}{l}\text { Inspection by } \\
\text { optical microscope }\end{array}$ & $\begin{array}{l}\text { NL-CR / Nikon Microscope } \\
\text { Dedicated microscope for lithography inspection }\end{array}$ \\
\hline 5 & $\begin{array}{l}\text { Plasma etching of Si } \\
\text { B-HARS } \\
\text { (\#etch158) }\end{array}$ & $\begin{array}{l}\text { NL-CR / Adixen AMS } 100 \mathrm{SE} \\
\text { Program: B-HARS } \\
\text { - } \mathrm{SF}_{6} \text { flow: } 250 \mathrm{sccm} \\
\text { - } \mathrm{C}_{4} \mathrm{~F}_{8} \text { flow: } 200 \mathrm{sccm} \\
\text { - Time: } 3 / 1 \mathrm{~s} \\
\text { - Priority: } 2 / 1 \\
\text { - ICP: } 1500 \mathrm{~W} \\
\text { - CCP (pulsed LF): } 80 \mathrm{~W} \\
\text { - On/off: } 10 / 90 \mathrm{~ms} \\
\text { - SH: } 200 \mathrm{~mm}\end{array}$ \\
\hline
\end{tabular}


- APC: $100 \%$

- He: $10 \mathrm{mbar}$

- Electrode: $10^{\circ} \mathrm{C}$

- Etch-rate Si: $1-5 \mu \mathrm{m} \mathrm{min}^{-1}$

- Etch-rate PR: $25-50 \mathrm{~nm} \mathrm{~min}^{-1}$

Thickness: $25 \mu \mathrm{m}$

Etch-time: $\sim 10 \mathrm{~min}$

\# of dummies: 1

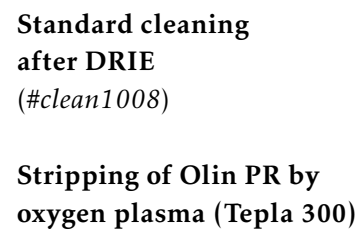

Stripping resist/cleaning in "Piranha" private use

Dry oxidation of Silicon at $800^{\circ} \mathrm{C}$

Etching in HF 1\% (private use)
CR-NL

Removal of Olin PR and

FluoroCarbon residue (metal free)

CR-NL / Tepla 300

Barrel Etcher $(2.45 \mathrm{GHz})$

Ultra clean system only (no metals except Al)

- See list with recipes in cleanroom

- $\mathrm{O}_{2}$ flow: $200 \mathrm{sccm}(50 \%)$

- Power: up to $1000 \mathrm{~W}$

- Pressure: $1 \mathrm{mbar}$

- Time: $20 \mathrm{~min}$

NL-CR / WB 9

$\mathrm{H}_{2} \mathrm{SO}_{4}: \mathrm{H}_{2} \mathrm{O}_{2}(3: 1)$ vol\%

- Add $\mathrm{H}_{2} \mathrm{O}_{2}$ slowly(!) to $\mathrm{H}_{2} \mathrm{SO}_{4}$; exothermic process!

- Adjust the hotplate temperature to $85^{\circ} \mathrm{C}$, the temperature will increase to $130^{\circ} \mathrm{C}$

- Cleaning temperature: $130^{\circ} \mathrm{C}$

- Time: $20 \mathrm{~min}$

- Quick Dump Rinse: $<0.1 \mu S$

- Spin drying

NL-CR / Furnace B3

Standby temperature: $800^{\circ} \mathrm{C}$

- Program: 0X800C

- Temperature: $800^{\circ} \mathrm{C}$

- Gas: $\mathrm{O}_{2}$

- Flow: $2 \mathrm{~L} \mathrm{~min}^{-1}$

- Time: $30 \mathrm{~min}$

NL-CR / WB 9

Use private beaker HF $1 \%$ standard

- Time: $10 \mathrm{~min}$ (etch until hydrofobic surface) 
- Quick Dump Rinse: $<0.1 \mu S$

- Spin drying

\section{E.5.4 LPCVD of $\mathrm{Si}_{3} \mathrm{~N}_{4}$}

\begin{tabular}{|c|c|c|}
\hline Step & Process & \\
\hline 7 & $\begin{array}{l}\text { Standard cleaning } \mathrm{HNO}_{3} \\
(\# \text { clean1001) }\end{array}$ & $\begin{array}{l}\text { NL-CR-WB14 } \\
\mathrm{HNO}_{3}(99 \%) \text { Selectiepur: MERCK } \\
\mathrm{HNO}_{3}(69 \%) \text { VLSI: MERCK } \\
\text { - Beaker 1: fumic } \mathrm{HNO}_{3}(99 \%), 5 \mathrm{~min} \\
\text { - Beaker 2: fumic } \mathrm{HNO}_{3}(99 \%), 5 \mathrm{~min} \\
\text { - Quick Dump Rinse: }<0.1 \mu \mathrm{S} \\
\text { - Beaker 3: boiling }\left(95^{\circ} \mathrm{C}\right) \mathrm{HNO} 3(69 \%), 10 \mathrm{~min} \\
\text { - Quick Dump Rinse: }<0.1 \mu \mathrm{S} \\
\text { - Spin drying }\end{array}$ \\
\hline 8 & $\begin{array}{l}\text { Dry oxidation and annealing } \\
@ 950^{\circ} \mathrm{C} \text { of silicon } \\
(\# f i l m 171)\end{array}$ & $\begin{array}{l}\text { NL-CR / Furnace B3 } \\
\text { Standby temperature: } 800^{\circ} \mathrm{C} \\
\text { - Program: } 0 \times 950 \mathrm{C} \\
\text { - Temperature: } 950^{\circ} \mathrm{C} \\
\text { - Gasses: } \mathrm{O}_{2} \\
\text { - Stress: } \sim 300 \mathrm{MPa}[\mathrm{C}] \\
\text { - Flow: } 2 \mathrm{~L} \mathrm{~min}^{-1} \\
\text { - Ramp: } 10^{\circ} \mathrm{Cmin}^{-1} \\
\text { - Cooldown: } 7.5^{\circ} \mathrm{Cmin}^{-1} \\
\text { Thickness: } 50 \mathrm{~nm} \\
\text { Annealing time: } 1 \mathrm{~h} \\
\text { \# of dummies: } 1\end{array}$ \\
\hline 9 & $\begin{array}{l}\text { Ellipsometer } \\
\text { measurement } \\
(\# \text { metro107) }\end{array}$ & $\begin{array}{l}\text { NL-CR / Plasmos and Woollam Ellipsometer } \\
\text { First Woollam: } \\
\text { - Cauchy model } \\
\text { - Fit on } A, B \text { and } k \\
\text { - Read } N_{\mathrm{f}} \text { and layer thickness } t \\
\text { Then Plasmos: } \\
\text { - Use } N_{\mathrm{f}} \text { and } t \text { from Woollam } \\
\text { - Raster } 7 \times 7(-41,-41)-(41,41) \\
\text { - Wafer diameter } 100 \mathrm{~mm} \text {, edge } 5.0 \mathrm{~mm} \\
\text { - } 37 \text { measurement points }\end{array}$ \\
\hline
\end{tabular}


10 Standard cleaning $\mathrm{HNO}_{3}$ (\#clean1001)
NL-CR-WB14

$\mathrm{HNO}_{3}$ (99\%) Selectiepur: MERCK

$\mathrm{HNO}_{3}(69 \%)$ VLSI: MERCK

- Beaker 1: fumic $\mathrm{HNO}_{3}$ (99\%), 5 min

- Beaker 2: fumic $\mathrm{HNO}_{3}$ (99\%), 5 min

- Quick Dump Rinse: $<0.1 \mu \mathrm{S}$

- Beaker 3: boiling $\left(95^{\circ} \mathrm{C}\right) \mathrm{HNO}_{3}(69 \%), 10 \mathrm{~min}$

- Quick Dump Rinse: $<0.1 \mu \mathrm{S}$

- Spin drying

\section{$11 \quad$ LPCVD $\mathrm{Si}_{3} \mathrm{~N}_{4}$ Stoichiometric}

(\#film 101)
NL-CR / Tempress LPCVD F1

A flush purge of $2.5 \mathrm{~h}$ is needed before deposition Tube: F1

- Program: Nitr01

- $\mathrm{SiH}_{2} \mathrm{Cl}_{2}$ flow: $22 \mathrm{sccm}$

- $\mathrm{NH}_{3}$ flow: $66 \mathrm{sccm}$

- Temperature: $800^{\circ} \mathrm{C}$

- Pressure: $200 \mathrm{mtorr}$

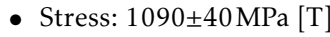

- Deposition rate: $4-6 \mathrm{~nm} \mathrm{~min}^{-1}$

- $\mathrm{N}_{\mathrm{f}}: 2.008$

Thickness: $150 \mathrm{~nm}$

Deposition time: $\sim 30 \mathrm{~min}$

\# of dummies: 1
Optical inspection of LPCVD layers

(\#metro013)
NL-CR / Cold Light Source

Particle inspection using Cold Light Source Layers: TEOS $\mathrm{SiO}_{2}, \mathrm{SiO}_{2}, \mathrm{Si}_{3} \mathrm{~N}_{4}$, poly-Si

Procedure: Check wafer for particles using streaking light

\section{3 \\ Ellipsometer measurement}

(\#metro107)
NL-CR / Plasmos and Woollam Ellipsometer

First Woollam:

- Cauchy model

- Fit on $A, B$ and $k$

- $\operatorname{Read} N_{\mathrm{f}}$ and layer thickness $t$

Then Plasmos:

- Use $N_{\mathrm{f}}$ and $t$ from Woollam

- Raster 7x7 (-41,-41) - $(41,41)$

- Wafer diameter $100 \mathrm{~mm}$, edge $5.0 \mathrm{~mm}$ 


\section{E.5.5 LPCVD of poly-Si and annealing}

\begin{tabular}{|c|c|c|}
\hline Step & Process & \\
\hline 14 & $\begin{array}{l}\text { Micro balance } \\
\text { measurement } \\
(\# \text { metro106) }\end{array}$ & NL-CR / Satorius Micro Balance \\
\hline 15 & $\begin{array}{l}\text { Standard cleaning } \mathrm{HNO}_{3} \\
(\# \text { clean } 1001)\end{array}$ & $\begin{array}{l}\text { NL-CR-WB14 } \\
\mathrm{HNO}_{3}(99 \%) \text { Selectiepur: MERCK } \\
\mathrm{HNO}_{3}(69 \%) \text { VLSI: MERCK } \\
\text { - Beaker 1: fumic } \mathrm{HNO}_{3}(99 \%), 5 \mathrm{~min} \\
\text { - Beaker 2: fumic } \mathrm{HNO}_{3}(99 \%), 5 \mathrm{~min} \\
\text { - Quick Dump Rinse: }<0.1 \mu \mathrm{S} \\
\text { - Beaker 3: boiling }\left(95^{\circ} \mathrm{C}\right) \mathrm{HNO} 3(69 \%), 10 \mathrm{~min} \\
\text { - Quick Dump Rinse: }<0.1 \mu \mathrm{S} \\
\text { - Spin drying }\end{array}$ \\
\hline 16 & $\begin{array}{l}\text { LPCVD Poly-Si }-\mathbf{5 9 0}{ }^{\circ} \mathbf{C} \\
(\# f i l m 105)\end{array}$ & $\begin{array}{l}\text { NL-CR / Tempress LPCVD } \\
\text { Tube: F2 } \\
\text { Program: senspoly } \\
\text { - } \mathrm{SiH}_{4} \text { flow: } 50 \mathrm{sccm} \\
\text { - Temperature: } 590^{\circ} \mathrm{C} \\
\text { - Pressure: } 250 \mathrm{mtorr} \\
\text { - Deposition rate: } 3.7 \mathrm{~nm} \mathrm{~min}^{-1} \\
\text { - Stress: } 30 \mathrm{MPa}[\mathrm{T}] \\
\text { Put dummy in centre of furnace } \\
\text { Thickness: } 1.8 \mu \mathrm{m} \\
\text { Deposition time: } 8 \mathrm{~h} \\
\text { \# of dummies: } 3\end{array}$ \\
\hline 17 & $\begin{array}{l}\text { Annealing at } \\
1050^{\circ} \mathrm{C} \text { with } \mathrm{N}_{2} \\
(\# \text { therm } 105)\end{array}$ & $\begin{array}{l}\text { NL-CR / Furnace B3 } \\
\text { Standby temperature: } 800^{\circ} \mathrm{C} \\
\text { - Program: ANN } 1050 \mathrm{C} \\
\text { - Temperature: } 1050{ }^{\circ} \mathrm{C} \\
\text { - Gas: } \mathrm{N}_{2} \\
\text { - Flow: } 1 \mathrm{~L} \mathrm{~min}^{-1} \\
\text { - Ramp: } 10^{\circ} \mathrm{Cmin}^{-1}\end{array}$ \\
\hline
\end{tabular}


Annealing time: $1 \mathrm{~h}$

\begin{tabular}{lll}
\hline 18 & $\begin{array}{l}\text { Inspection by } \\
\text { optical microscope } \\
(\# m e t r o 101)\end{array}$ & $\begin{array}{l}\text { NL-CR / Nikon Microscope } \\
\text { Dedicated microscope for lithography inspection }\end{array}$ \\
\hline 19 & $\begin{array}{l}\text { Micro balance } \\
\text { measurement } \\
\text { (\#metro106) }\end{array}$ & NL-CR / Satorius Micro Balance \\
\hline
\end{tabular}

\section{E.5.6 Partial oxidation of polysilicon}

\begin{tabular}{|c|c|c|}
\hline Step & Process & \\
\hline 20 & $\begin{array}{l}\text { Standard cleaning } \mathrm{HNO}_{3} \\
(\# \text { clean 1001) }\end{array}$ & $\begin{array}{l}\text { NL-CR-WB14 } \\
\mathrm{HNO}_{3}(99 \%) \text { Selectiepur: MERCK } \\
\mathrm{HNO}_{3}(69 \%) \text { VLSI: MERCK } \\
\text { - Beaker 1: fumic } \mathrm{HNO}_{3}(99 \%), 5 \mathrm{~min} \\
\text { - Beaker 2: fumic } \mathrm{HNO}_{3}(99 \%), 5 \mathrm{~min} \\
\text { - Quick Dump Rinse: }<0.1 \mu \mathrm{S} \\
\text { - Beaker 3: boiling }\left(95^{\circ} \mathrm{C}\right) \mathrm{HNO} 3(69 \%), 10 \mathrm{~min} \\
\text { - Quick Dump Rinse: }<0.1 \mu \mathrm{S} \\
\text { - Spin drying }\end{array}$ \\
\hline 21 & 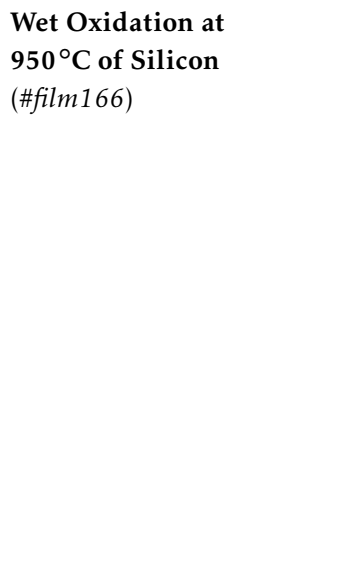 & $\begin{array}{l}\text { NL-CR / Furnace B2 } \\
\text { Standby temperature: } 800^{\circ} \mathrm{C} \\
\text { Check water level of bubbler } \\
\text { - Program: WET950C } \\
\text { - Temperature: } 950{ }^{\circ} \mathrm{C} \\
\text { - Gas: } \mathrm{H}_{2} \mathrm{O}+\mathrm{N}_{2}(\mathrm{Bubbler}) \\
\text { - Stress: } \sim 300 \mathrm{MPa}[\mathrm{C}] \\
\text { - Flow: } 2 \mathrm{~L} \mathrm{~min}^{-1} \\
\text { - Ramp: } 10^{\circ} \mathrm{Cmin}^{-1} \\
\text { - Cooldown: } 7.5^{\circ} \mathrm{Cmin} \mathrm{min}^{-1} \\
\text { Thickness: } 550 \mathrm{~nm} \\
\text { Oxidation time: } 1 \mathrm{~h} \\
\text { \# of dummies: } 1\end{array}$ \\
\hline 22 & $\begin{array}{l}\text { Ellipsometer } \\
\text { measurement } \\
(\# \text { metro107) }\end{array}$ & $\begin{array}{l}\text { NL-CR / Plasmos and Woollam Ellipsometer } \\
\text { First Woollam: } \\
\text { - Cauchy model }\end{array}$ \\
\hline
\end{tabular}


- Fit on $A, B$ and $k$

- Read $N_{\mathrm{f}}$ and layer thickness $t$

Then Plasmos:

- Use $N_{\mathrm{f}}$ and $t$ from Woollam

- Raster 7x7 (-41,-41) - (41,41)

- Wafer diameter $100 \mathrm{~mm}$, edge $5.0 \mathrm{~mm}$

- 37 measurement points

\begin{tabular}{|c|c|c|}
\hline 23 & $\begin{array}{l}\text { Etching BHF (1:7) } \\
\mathrm{SiO}_{2} \\
(\# \text { etch124) }\end{array}$ & $\begin{array}{l}\text { NL-CR-WB15 } \\
\text { Use dedicated beaker BHF (1:7) metal free } \\
\text { - Quick Dump Rinse: }<0.1 \mu \mathrm{S} \\
\text { - Spin drying } \\
\text { Etch-rate thermal } \mathrm{SiO}_{2}=60-80 \mathrm{~nm} \mathrm{~min}^{-1} \\
\text { Etch-rate PECVD } \mathrm{SiO}_{2}=125 \mathrm{~nm} \mathrm{~min}^{-1} \\
\text { Etch-rate TEOS } \mathrm{SiO}_{2}=180 \mathrm{~nm} \mathrm{~min}^{-1} \\
\text { Thickness: } 700 \mathrm{~nm} \\
\text { Etch-time: } 8 \mathrm{~min} \\
\text { \# of dummies: } 1\end{array}$ \\
\hline 24 & $\begin{array}{l}\text { Micro balance } \\
\text { measurement } \\
(\# \text { metro106) }\end{array}$ & NL-CR / Satorius Micro Balance \\
\hline
\end{tabular}

\section{E.5.7 Patterning of the device layer - I (SACRI)}

\begin{tabular}{lll}
\hline Step & Process & \\
\hline 25 & Standard cleaning $\mathrm{HNO}_{3}$ & $\mathrm{NL}_{3}-\mathrm{CR}-\mathrm{WB} 14$ \\
& (\#clean1001) & $\mathrm{HNO}_{3}(99 \%)$ Selectiepur: MERCK \\
& $\mathrm{HNO}_{3}(69 \%)$ VLSI: MERCK \\
& - Beaker 1: fumic $\mathrm{HNO}_{3}(99 \%), 5 \mathrm{~min}$ \\
& - Beaker 2: fumic $\mathrm{HNO}_{3}(99 \%), 5 \mathrm{~min}$ \\
& - Quick Dump Rinse: $<0.1 \mu \mathrm{S}$ \\
& - Beaker 3: boiling $\left(95^{\circ} \mathrm{C}\right) \mathrm{HNO}_{3}(69 \%), 10 \mathrm{~min}$ \\
& - Quick Dump Rinse: $<0.1 \mu \mathrm{S}$ \\
& - Spin drying \\
& \\
& & \\
\hline 26 & Standard lithography & NL-CR \\
& Olin 907-17 & Continue immediately with \\
& priming the step!
\end{tabular}


Dehydration bake

Priming (HMDS)

Coating (OLIN 907-17)

Alignment \& Exposure

(Olin 907-17)

Development (Olin 907-17)

Postbake (Olin 907-17)

Inspection by optical microscope
NL-CR / WB 21-22

- Hotplate: $120^{\circ} \mathrm{C}: 5 \mathrm{~min}$

Spincoater

- Spin program: 4 (4000 rpm, 30 s)

Primus Spinner

- Spin program: $4(4000 \mathrm{rpm}, 30 \mathrm{~s}, 1.7 \mu \mathrm{m})$

- Prebake $\left(95^{\circ} \mathrm{C}\right): 90 \mathrm{~s}$

Electronic Vision Group 620 Mask Aligner

- Hg-lamp: $12 \mathrm{~mW} \mathrm{~cm}^{-2}$

- Exposure time: $3.8 \mathrm{~s}$

- Mask: SACRI

Developer: OPD4262

- After exposure bake $\left(120^{\circ} \mathrm{C}\right): 60 \mathrm{~s}$

- Time: $30 \mathrm{~s}$ in beaker 1

- Time: $15-30 \mathrm{~s}$ in beaker 2

- Quick Dump Rinse: $<0.1 \mu \mathrm{S}$

- Spin drying

- Hotplate: $120^{\circ} \mathrm{C}: 0 \mathrm{~min}$

NL-CR / Nikon Microscope

Dedicated microscope for lithography inspection

\section{Plasma etching of $\mathrm{Si}$ B-HARS}

(\#etch158)
NL-CR / Adixen AMS 100 SE

Program: B-HARS

- $\mathrm{SF}_{6}$ flow: $250 \mathrm{sccm}$

- $\mathrm{C}_{4} \mathrm{~F}_{8}$ flow: $200 \mathrm{sccm}$

- Time: $3 / 1 \mathrm{~s}$

- Priority: $2 / 1$

- ICP: $1500 \mathrm{~W}$

- CCP (pulsed LF): $80 \mathrm{~W}$

- On/off: $10 / 90 \mathrm{~ms}$

- $\mathrm{SH}: 200 \mathrm{~mm}$

- APC: $100 \%$

- He: $10 \mathrm{mbar}$

- Electrode: $10^{\circ} \mathrm{C}$

- Etch-rate Si: $1-5 \mu \mathrm{m} \mathrm{min}{ }^{-1}$

- Etch-rate PR: 25-50 $\mathrm{nm} \mathrm{min}^{-1}$ 
Thickness: $1.5 \mu \mathrm{m}$

Etch-time: $\sim 1 \mathrm{~min}$

\# of dummies: 1

\begin{tabular}{|c|c|c|}
\hline 28 & $\begin{array}{l}\text { Standard cleaning } \\
\text { after DRIE } \\
(\# \text { clean } 1008)\end{array}$ & $\begin{array}{l}\text { CR-NL } \\
\text { Removal of Olin PR and } \\
\text { FluoroCarbon residue (metal free) }\end{array}$ \\
\hline & $\begin{array}{l}\text { Stripping of Olin PR by } \\
\text { oxygen plasma (Tepla 300) }\end{array}$ & $\begin{array}{l}\text { CR-NL / Tepla } 300 \\
\text { Barrel Etcher ( } 2.45 \mathrm{GHz}) \\
\text { Ultra clean system only (no metals except Al) } \\
\text { - See list with recipes in cleanroom } \\
\text { - } \mathrm{O}_{2} \text { flow: } 200 \mathrm{sccm}(50 \%) \\
\text { - Power: up to } 1000 \mathrm{~W} \\
\text { - Pressure: } 1 \mathrm{mbar} \\
\text { - Time: } 20 \mathrm{~min}\end{array}$ \\
\hline & $\begin{array}{l}\text { Stripping resist/cleaning } \\
\text { in "Piranha" private use }\end{array}$ & $\begin{array}{l}\mathrm{NL}-\mathrm{CR} / \mathrm{WB} 9 \\
\mathrm{H}_{2} \mathrm{SO}_{4}: \mathrm{H}_{2} \mathrm{O}_{2}(3: 1) \text { vol\% } \\
\text { - Add } \mathrm{H}_{2} \mathrm{O}_{2} \text { slowly(!) to } \mathrm{H}_{2} \mathrm{SO}_{4} \text {; exothermic process! } \\
\text { - Adjust the hotplate temperature to } 85^{\circ} \mathrm{C} \text {, the } \\
\text { temperature will increase to } 130^{\circ} \mathrm{C} \\
\text { - Cleaning temperature: } 130^{\circ} \mathrm{C} \\
\text { - Time: } 20 \text { min } \\
\text { - Quick Dump Rinse: }<0.1 \mu \mathrm{S} \\
\text { - Spin drying }\end{array}$ \\
\hline & $\begin{array}{l}\text { Dry oxidation of } \\
\text { Silicon at } 800^{\circ} \mathrm{C}\end{array}$ & $\begin{array}{l}\text { NL-CR / Furnace B3 } \\
\text { Standby temperature: } 800^{\circ} \mathrm{C} \\
\text { - Program: } 0 \times 800 \mathrm{C} \\
\text { - Temperature: } 800^{\circ} \mathrm{C} \\
\text { - Gas: } \mathrm{O}_{2} \\
\text { - Flow: } 2 \mathrm{~L} \mathrm{~min}-1 \\
\text { - Time: } 30 \mathrm{~min}^{-1}\end{array}$ \\
\hline & $\begin{array}{l}\text { Etching in HF } 1 \% \\
\text { (private use) }\end{array}$ & $\begin{array}{l}\text { NL-CR / WB } 9 \\
\text { Use private beaker HF } 1 \% \text { standard } \\
\text { - Time: } 10 \text { min (etch until hydrofobic surface) } \\
\text { - Quick Dump Rinse: }<0.1 \mu \mathrm{S} \\
\text { - Spin drying }\end{array}$ \\
\hline
\end{tabular}

\section{E.5.8 Thermal oxidation - I}




\begin{tabular}{|c|c|c|}
\hline Step & Process & \\
\hline 29 & $\begin{array}{l}\text { Standard cleaning } \mathrm{HNO}_{3} \\
(\# \text { clean } 1001)\end{array}$ & $\begin{array}{l}\text { NL-CR-WB14 } \\
\mathrm{HNO}_{3}(99 \%) \text { Selectiepur: MERCK } \\
\mathrm{HNO}_{3}(69 \%) \text { VLSI: MERCK } \\
\text { - Beaker 1: fumic } \mathrm{HNO}_{3}(99 \%), 5 \mathrm{~min} \\
\text { - Beaker 2: fumic } \mathrm{HNO}_{3}(99 \%), 5 \mathrm{~min} \\
\text { - Quick Dump Rinse: }<0.1 \mu \mathrm{S} \\
\text { - Beaker 3: boiling }\left(95^{\circ} \mathrm{C}\right) \mathrm{HNO} 3(69 \%), 10 \mathrm{~min} \\
\text { - Quick Dump Rinse: }<0.1 \mu \mathrm{S} \\
\text { - Spin drying }\end{array}$ \\
\hline
\end{tabular}

\begin{tabular}{|c|c|c|}
\hline 30 & $\begin{array}{l}\text { Dry oxidation and annealing } \\
\text { @ } 950^{\circ} \mathrm{C} \text { of silicon } \\
(\# f i l m 171)\end{array}$ & $\begin{array}{l}\text { NL-CR / Furnace B3 } \\
\text { Standby temperature: } 800{ }^{\circ} \mathrm{C} \\
\text { - Program: } 0 x 950 \mathrm{C} \\
\text { - Temperature: } 950{ }^{\circ} \mathrm{C} \\
\text { - Gasses: } \mathrm{O}_{2} \\
\text { - Stress: } \sim 300 \mathrm{MPa}[\mathrm{C}] \\
\text { - Flow: } 2 \mathrm{~L} \mathrm{~min}^{-1} \\
\text { - Ramp: } 10^{\circ} \mathrm{C} \mathrm{min}^{-1} \\
\text { - Cooldown: } 7.5^{\circ} \mathrm{Cmin} \min ^{-1} \\
\text { Thickness: } 50 \mathrm{~nm} \\
\text { Annealing time: } 1 \mathrm{~h} \\
\text { \# of dummies: } 1\end{array}$ \\
\hline 31 & $\begin{array}{l}\text { Ellipsometer } \\
\text { measurement } \\
(\# \text { metro107) }\end{array}$ & $\begin{array}{l}\text { NL-CR / Plasmos and Woollam Ellipsometer } \\
\text { First Woollam: } \\
\text { - Cauchy model } \\
\text { - Fit on } A, B \text { and } k \\
\text { - Read } N_{\mathrm{f}} \text { and layer thickness } t \\
\text { Then Plasmos: } \\
\text { - Use } N_{\mathrm{f}} \text { and } t \text { from Woollam } \\
\text { - Raster } 7 \mathrm{x} 7(-41,-41)-(41,41) \\
\text { - Wafer diameter } 100 \mathrm{~mm}, \text { edge } 5.0 \mathrm{~mm} \\
\text { - } 37 \text { measurement points }\end{array}$ \\
\hline
\end{tabular}

\section{E.5.9 LPCVD of SiRN (membranes and springs)}

\begin{tabular}{lll}
\hline Step & Process \\
\hline 32 & Standard cleaning $\mathrm{HNO}_{3}$ & NL-CR-WB14
\end{tabular}


(\#clean1001)

$\mathrm{HNO}_{3}$ (99\%) Selectiepur: MERCK

$\mathrm{HNO}_{3}(69 \%)$ VLSI: MERCK

- Beaker 1: fumic $\mathrm{HNO}_{3}$ (99\%), 5 min

- Beaker 2: fumic $\mathrm{HNO}_{3}$ (99\%), 5 min

- Quick Dump Rinse: $<0.1 \mu \mathrm{S}$

- Beaker 3: boiling $\left(95^{\circ} \mathrm{C}\right) \mathrm{HNO}_{3}(69 \%), 10 \mathrm{~min}$

- Quick Dump Rinse: $<0.1 \mu \mathrm{S}$

- Spin drying

\begin{tabular}{|c|c|c|}
\hline 33 & $\begin{array}{l}\text { LPCVD of SiRN } \\
(\mathbf{5 0 - 1 0 0 ~ M P a ~}) \\
(\# f i l m 143)\end{array}$ & $\begin{array}{l}\text { NL-CR / Tempress LPCVD } \\
\text { Program: SiRN01 } \\
\text { Tube: G3 } \\
\text { - } \mathrm{SiH}_{2} \mathrm{Cl}_{2} \text { flow: } 77.5 \mathrm{sccm} \\
\text { - } \mathrm{NH}_{3} \text { flow: } 20 \mathrm{sccm} \\
\text { - Temperature: } 820^{\circ} \mathrm{C}, 850^{\circ} \mathrm{C} \text { and } 870^{\circ} \mathrm{C} \\
\text { - Pressure: } 150 \mathrm{mtorr} \\
\text { - } \mathrm{N}_{2} \text { flow: } 250 \mathrm{sccm} \\
\text { - Deposition rate: } 4 \mathrm{~nm} \mathrm{~min}^{-1} \\
\text { - } \mathrm{N}_{\mathrm{f}}: 2.18 \\
\text { - Stress (range): } 50-100 \mathrm{MPa}[\mathrm{T}] \\
\text { Thickness: } 1 \mu \mathrm{m} \\
\text { Deposition time: } 4 \mathrm{~h} 10 \mathrm{~min} \\
\text { \# of dummies: } 1\end{array}$ \\
\hline 34 & $\begin{array}{l}\text { Ellipsometer } \\
\text { measurement } \\
\text { (\#metro107) }\end{array}$ & $\begin{array}{l}\text { NL-CR / Plasmos and Woollam Ellipsometer } \\
\text { First Woollam: } \\
\text { - Cauchy model } \\
\text { - Fit on } A, B \text { and } k \\
\text { - Read } N_{\mathrm{f}} \text { and layer thickness } t \\
\text { Then Plasmos: } \\
\text { - Use } N_{\mathrm{f}} \text { and } t \text { from Woollam } \\
\text { - Raster } 7 x 7(-41,-41)-(41,41) \\
\text { - Wafer diameter } 100 \mathrm{~mm}, \text { edge } 5.0 \mathrm{~mm} \\
\text { - } 37 \text { measurement points }\end{array}$ \\
\hline
\end{tabular}

\section{E.5.10 SiRN patterning (membranes, springs and bottom elec- trode contact)}

\begin{tabular}{lll}
\hline Step & Process \\
\hline 35 & Standard cleaning $\mathrm{HNO}_{3}$ & NL-CR-WB14
\end{tabular}


(\#clean1001)

$\mathrm{HNO}_{3}(99 \%)$ Selectiepur: MERCK

$\mathrm{HNO}_{3}(69 \%)$ VLSI: MERCK

- Beaker 1: fumic $\mathrm{HNO}_{3}$ (99\%), $5 \mathrm{~min}$

- Beaker 2: fumic $\mathrm{HNO}_{3}$ (99\%), 5 min

- Quick Dump Rinse: $<0.1 \mu \mathrm{S}$

- Beaker 3: boiling $\left(95^{\circ} \mathrm{C}\right) \mathrm{HNO}_{3}(69 \%), 10 \mathrm{~min}$

- Quick Dump Rinse: $<0.1 \mu \mathrm{S}$

- Spin drying

\section{Standard lithography Olin 908-35 \\ (\#lith1003) \\ Dehydration bake \\ Priming (HMDS) \\ Coating (OLIN 908-35) \\ Alignment \& Exposure \\ (Olin 908-35)}

Development (Olin 908-35)

Postbake (Olin 908-35)

Inspection by optical microscope

\section{NL-CR}

Continue immediately with priming the step!

NL-CR / WB 21-22

- Hotplate: $120^{\circ} \mathrm{C}: 5 \mathrm{~min}$

Spincoater

- Spin program: 4 (4000 rpm, 30s)

Primus Spinner

- Spin program: $4(4000 \mathrm{rpm}, 30 \mathrm{~s}, 3.5 \mu \mathrm{m})$

- Prebake $\left(95^{\circ} \mathrm{C}\right): 120 \mathrm{~s}$

Electronic Vision Group 620 Mask Aligner

- Hg-lamp: $12 \mathrm{~mW} \mathrm{~cm}^{-2}$

- Exposure time: $9 \mathrm{~s}$

- Mask: MEMBR1

Developer: OPD4262

- After exposure bake $\left(120^{\circ} \mathrm{C}\right): 60 \mathrm{~s}$

- Time: $30 \mathrm{~s}$ in beaker 1

- Time: $15-30$ s in beaker 2

- Quick Dump Rinse: $<0.1 \mu \mathrm{S}$

- Spin drying

- Hotplate: $120^{\circ} \mathrm{C}: 10 \mathrm{~min}$

NL-CR / Nikon Microscope

Dedicated microscope for lithography inspection 
(\#etch174)

- Argon flow: $100 \mathrm{sccm}$

- $\mathrm{CHF}_{3}$ flow: $100 \mathrm{sccm}$

- ICP: $1200 \mathrm{~W}$

- CCP: $150 \mathrm{~W}$

- SH: $200 \mathrm{~mm}$

- APC: $100 \%$

- He: $10 \mathrm{mbar}$

- Electrode: $-10^{\circ} \mathrm{C}$

- Etch-rate Si: 70-80 $\mathrm{nm} \mathrm{min}^{-1}$

- Etch-rate Olin PR: $160 \mathrm{~nm} \mathrm{~min}^{-1}$

- Etch-rate $\mathrm{SiO}_{2}$ : $250 \mathrm{~nm} \mathrm{~min}^{-1}$

- Etch-rate SiRN: $300 \mathrm{~nm} \mathrm{~min}^{-1}$

- Etch-rate SU-8: $150 \mathrm{~nm} \mathrm{~min}^{-1}$

Thickness: $>1.2 \mu \mathrm{m}$

Etch-time: $6 \mathrm{~min}$

\# of dummies: 0

$38 \quad$ Stripping of Olin PR by oxygen plasma (Tepla 300) (\#lith142)
CR 125 A / Tepla 300

Barrel Etcher $(2.45 \mathrm{GHz})$

Ultra clean system only (no metals except $\mathrm{Al}$ )

- See list with recipes in cleanroom

- $\mathrm{O}_{2}$ flow: $200 \mathrm{sccm}(50 \%)$

- Power: up to $1000 \mathrm{~W}$

- Pressure: $1 \mathrm{mbar}$

\begin{tabular}{ll}
\hline 39 Stripping of Olin & NL-CR-WB14 \\
PR - Standard & $\mathrm{HNO}_{3}(99 \%)$ \\
$(\# l i t h 116)$ & \\
& - Time: 20 min or $100 \%$ removal of PR \\
& - Quick Dump Rinse: $<0.1 \mu \mathrm{S}$ \\
& - Spin drying
\end{tabular}

$40 \quad$ Inspection by optical microscope

(\#metro101)
NL-CR / Nikon Microscope

Dedicated microscope for lithography inspection

\section{E.5.11 Thermal oxidation - II}

\begin{tabular}{lll}
\hline Step & Process \\
\hline 41 & Standard cleaning HNO $_{3}$ & $\mathrm{NL}^{-C R}-\mathrm{WB} 14$ \\
& $($ \#clean1001) & $\mathrm{HNO}_{3}(99 \%)$ Selectiepur: MERCK \\
& $\mathrm{HNO}_{3}(69 \%)$ VLSI: MERCK
\end{tabular}


- Beaker 1: fumic $\mathrm{HNO}_{3}$ (99\%), $5 \mathrm{~min}$

- Beaker 2: fumic $\mathrm{HNO}_{3}$ (99\%), 5 min

- Quick Dump Rinse: $<0.1 \mu \mathrm{S}$

- Beaker 3: boiling $\left(95^{\circ} \mathrm{C}\right) \mathrm{HNO}_{3}(69 \%), 10 \mathrm{~min}$

- Quick Dump Rinse: $<0.1 \mu \mathrm{S}$

- Spin drying

\begin{tabular}{|c|c|c|}
\hline 42 & $\begin{array}{l}\text { Dry oxidation and annealing } \\
\text { @ } 950^{\circ} \mathrm{C} \text { of silicon } \\
(\# f i l m 171)\end{array}$ & $\begin{array}{l}\text { NL-CR / Furnace B3 } \\
\text { Standby temperature: } 800^{\circ} \mathrm{C} \\
\text { - Program: } 0 x 950 \mathrm{C} \\
\text { - Temperature: } 950{ }^{\circ} \mathrm{C} \\
\text { - Gasses: } \mathrm{O}_{2} \\
\text { - Stress: } \sim 300 \mathrm{MPa}[\mathrm{C}] \\
\text { - Flow: } 2 \mathrm{~L} \mathrm{~min}^{-1} \\
\text { - Ramp: } 10^{\circ} \mathrm{C} \mathrm{min}^{-1} \\
\text { - Cooldown: } 7.5^{\circ} \mathrm{C} \mathrm{min}^{-1} \\
\text { Thickness: } 50 \mathrm{~nm} \\
\text { Annealing time: } 15 \mathrm{~h} \\
\text { \# of dummies: } 1\end{array}$ \\
\hline 43 & $\begin{array}{l}\text { Ellipsometer } \\
\text { measurement } \\
(\# \text { metro107) }\end{array}$ & $\begin{array}{l}\text { NL-CR / Plasmos and Woollam Ellipsometer } \\
\text { First Woollam: } \\
\text { - Cauchy model } \\
\text { - Fit on } A, B \text { and } k \\
\text { - Read } N_{\mathrm{f}} \text { and layer thickness } t \\
\text { Then Plasmos: } \\
\text { - Use } N_{\mathrm{f}} \text { and } t \text { from Woollam } \\
\text { - Raster } 7 \mathrm{x} 7(-41,-41)-(41,41) \\
\text { - Wafer diameter } 100 \mathrm{~mm} \text {, edge } 5.0 \mathrm{~mm} \\
\text { - } 37 \text { measurement points }\end{array}$ \\
\hline
\end{tabular}

\section{E.5.12 LPCVD of SiRN (support beams)}

\begin{tabular}{lll}
\hline Step & Process & \\
\hline 44 & $\begin{array}{l}\text { Standard cleaning } \mathrm{HNO}_{3} \\
\text { (\#clean1001) }\end{array}$ & $\mathrm{NL}-\mathrm{CR}-\mathrm{WB} 14$ \\
& $\mathrm{HNO}_{3}(99 \%)$ Selectiepur: MERCK \\
& $\mathrm{HNO}_{3}(69 \%)$ VLSI: MERCK \\
& - Beaker 1: fumic $\mathrm{HNO}_{3}(99 \%), 5 \mathrm{~min}$ \\
& - Beaker 2: fumic $\mathrm{HNO}_{3}(99 \%), 5 \mathrm{~min}$
\end{tabular}


- Quick Dump Rinse: $<0.1 \mu \mathrm{S}$

- Beaker 3: boiling $\left(95^{\circ} \mathrm{C}\right) \mathrm{HNO}_{3}(69 \%), 10 \mathrm{~min}$

- Quick Dump Rinse: $<0.1 \mu \mathrm{S}$

- Spin drying

\begin{tabular}{|c|c|c|}
\hline 45 & $\begin{array}{l}\text { LPCVD of SiRN } \\
\text { (50-100 MPa) } \\
\text { (\#film143) }\end{array}$ & $\begin{array}{l}\text { NL-CR / Tempress LPCVD } \\
\text { Program: SiRN01 } \\
\text { Tube: } \mathrm{G} 3 \\
\text { - } \mathrm{SiH}_{2} \mathrm{Cl}_{2} \text { flow: } 77.5 \mathrm{sccm} \\
\text { - } \mathrm{NH}_{3} \text { flow: } 20 \mathrm{sccm} \\
\text { - Temperature: } 820^{\circ} \mathrm{C}, 850^{\circ} \mathrm{C} \text { and } 870^{\circ} \mathrm{C} \\
\text { - Pressure: } 150 \mathrm{mtorr} \\
\text { - } \mathrm{N}_{2} \text { flow: } 250 \mathrm{sccm} \\
\text { - Deposition rate: } 4 \mathrm{~nm} \mathrm{~min}^{-1} \\
\text { - } \mathrm{N}_{\mathrm{f}}: 2.18 \\
\text { - Stress (range): } 50-100 \mathrm{MPa}[\mathrm{T}] \\
\text { Thickness: } 200 \mathrm{~nm} \\
\text { Deposition time: } 50 \mathrm{~min} \\
\text { \# of dummies: } 1\end{array}$ \\
\hline 46 & $\begin{array}{l}\text { Ellipsometer } \\
\text { measurement } \\
(\# \text { metro107) }\end{array}$ & $\begin{array}{l}\text { NL-CR / Plasmos and Woollam Ellipsometer } \\
\text { First Woollam: } \\
\text { - Cauchy model } \\
\text { - Fit on } A, B \text { and } k \\
\text { - Read } N_{\mathrm{f}} \text { and layer thickness } t \\
\text { Then Plasmos: } \\
\text { - Use } N_{\mathrm{f}} \text { and } t \text { from Woollam } \\
\text { - Raster } 7 \mathrm{x} 7(-41,-41)-(41,41) \\
\text { - Wafer diameter } 100 \mathrm{~mm} \text {, edge } 5.0 \mathrm{~mm} \\
\text { - } 37 \text { measurement points }\end{array}$ \\
\hline
\end{tabular}

\section{E.5.13 SiRN patterning (support beams)}

\begin{tabular}{lll}
\hline Step & Process & \\
\hline 47 & $\begin{array}{l}\text { Standard cleaning } \mathrm{HNO}_{3} \\
\text { (\#clean1001) }\end{array}$ & $\mathrm{NL}-\mathrm{CR}-\mathrm{WB} 14$ \\
& $\mathrm{HNO}_{3}(99 \%)$ Selectiepur: MERCK \\
& $\mathrm{HNO}_{3}(69 \%)$ VLSI: MERCK \\
& \\
& - Beaker 1: fumic $\mathrm{HNO}_{3}(99 \%), 5 \mathrm{~min}$ \\
& - Beaker 2: fumic $\mathrm{HNO}_{3}(99 \%), 5 \mathrm{~min}$ \\
& - Quick Dump Rinse: $<0.1 \mu \mathrm{S}$
\end{tabular}


- Beaker 3: boiling $\left(95^{\circ} \mathrm{C}\right) \mathrm{HNO}_{3}(69 \%), 10 \mathrm{~min}$

- Quick Dump Rinse: $<0.1 \mu \mathrm{S}$

- Spin drying

\begin{tabular}{l}
\hline $48 \quad \begin{array}{l}\text { Standard lithography } \\
\text { Olin 907-17 } \\
(\# l i t h 1002)\end{array}$ \\
Dehydration bake \\
Priming (HMDS) \\
Coating (OLIN 907-17) \\
\\
Alignment \& Exposure \\
(Olin 907-17)
\end{tabular}

NL-CR

Continue immediately with

priming the step!

NL-CR / WB 21-22

- Hotplate: $120^{\circ} \mathrm{C}: 5 \mathrm{~min}$

Spincoater

- Spin program: 4 (4000 rpm, 30s)

Primus Spinner

- Spin program: 4 (4000 rpm, $30 \mathrm{~s}, 1.7 \mu \mathrm{m})$

- Prebake $\left(95^{\circ} \mathrm{C}\right): 90 \mathrm{~s}$

Electronic Vision Group 620 Mask Aligner

- Hg-lamp: $12 \mathrm{~mW} \mathrm{~cm}^{-2}$

- Exposure time: $3.8 \mathrm{~s}$

- Mask: MEMBR2

Development (Olin 907-17) Developer: OPD4262

- After exposure bake $\left(120^{\circ} \mathrm{C}\right): 60 \mathrm{~s}$

- Time: $30 \mathrm{~s}$ in beaker 1

- Time: $15-30 \mathrm{~s}$ in beaker 2

- Quick Dump Rinse: $<0.1 \mu \mathrm{S}$

- Spin drying

Postbake (Olin 907-17)

- Hotplate: $120^{\circ} \mathrm{C}: 10 \mathrm{~min}$

Inspection by

NL-CR / Nikon Microscope

optical microscope

Dedicated microscope for lithography inspection

\begin{tabular}{ll}
\hline 99 & $\begin{array}{l}\text { Plasma etching of } \\
\text { multilayers } \\
(\# \text { etch174) }\end{array}$
\end{tabular}

NL-CR / Adixen AMS 100 SE

Program: ...

- Argon flow: $100 \mathrm{sccm}$

- $\mathrm{CHF}_{3}$ flow: $100 \mathrm{sccm}$

- ICP: $1200 \mathrm{~W}$

- CCP: $150 \mathrm{~W}$

- SH: $200 \mathrm{~mm}$ 
- APC: $100 \%$

- He: $10 \mathrm{mbar}$

- Electrode: $-10^{\circ} \mathrm{C}$

- Etch-rate Si: 70-80 $\mathrm{nm} \mathrm{min}^{-1}$

- Etch-rate Olin PR: $160 \mathrm{~nm} \mathrm{~min}^{-1}$

- Etch-rate $\mathrm{SiO}_{2}: 250 \mathrm{~nm} \mathrm{~min}^{-1}$

- Etch-rate SiRN: $300 \mathrm{~nm} \mathrm{~min}^{-1}$

- Etch-rate SU-8: $150 \mathrm{~nm} \mathrm{~min}^{-1}$

Thickness: $>250 \mathrm{~nm}$

Etch-time: $1 \mathrm{~min}$

\# of dummies: 0

\begin{tabular}{lll}
\hline 50 & $\begin{array}{l}\text { Stripping of Olin PR by } \\
\text { oxygen plasma (Tepla 300) } \\
\text { (\#lith142) }\end{array}$ & Barrel Etcher $(2.45 \mathrm{GHz})$ \\
& Ultra clean system only (no metals except Al) \\
& - See list with recipes in cleanroom \\
& - O flow: $200 \mathrm{sccm}(50 \%)$ \\
& - Power: up to $1000 \mathrm{~W}$ \\
& - Pressure: 1 mbar
\end{tabular}

51

Stripping of Olin
PR - Standard
$(\#$ Itith116)

\section{NL-CR-WB14}

$\mathrm{HNO}_{3}(99 \%)$

- Time: $20 \mathrm{~min}$ or $100 \%$ removal of PR

- Quick Dump Rinse: $<0.1 \mu \mathrm{S}$

- Spin drying

\begin{tabular}{ll}
\hline 52 & $\begin{array}{l}\text { Inspection by } \\
\text { optical microscope } \\
(\# m e t r o 101)\end{array}$
\end{tabular}

NL-CR / Nikon Microscope

Dedicated microscope for lithography inspection

\section{E.5.14 Sputtering of Al}

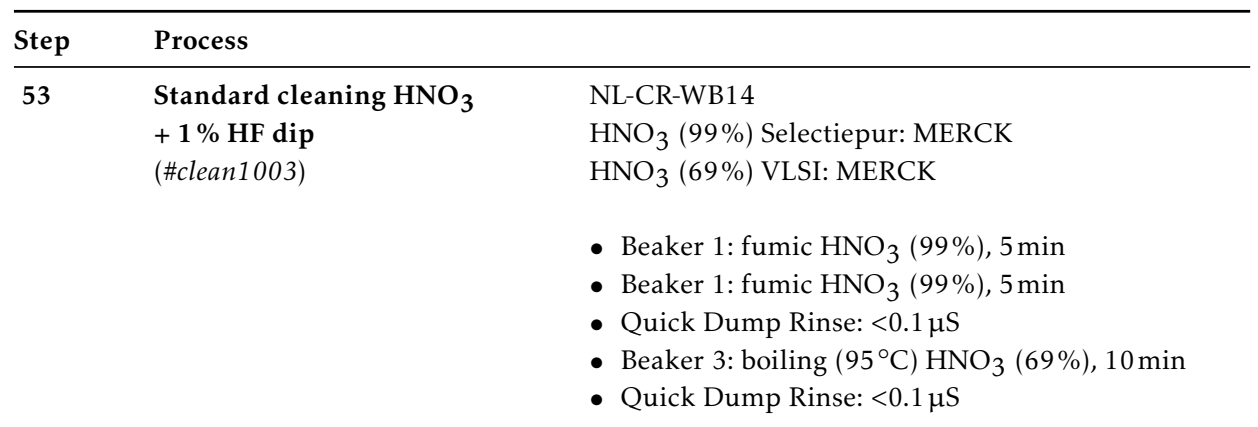


- Beaker 4: HF (1\%), 1 min

- Quick Dump Rinse: $<0.1 \mu \mathrm{S}$

- Spin drying

\begin{tabular}{ll}
\hline 54 & NL-CR / Oxford PL 400 \\
$\begin{array}{l}\text { Sesist compatible (Oxford) } \\
(\# f i l m 129)\end{array}$ & Program: $100 \mathrm{~nm} \mathrm{Al} 700 \mathrm{~W}$ \\
& - Power: $700 \mathrm{~W}$ \\
& - Pressure: $10 \mathrm{mtorr}$ \\
& - Deposition rate: $82 \mathrm{~nm} \mathrm{~min}^{-1}$ \\
& \\
& Thickness: $80 \mathrm{~nm}$ \\
& Deposition time: $1 \mathrm{~min}$ \\
& \# of dummies:
\end{tabular}

\section{E.5.15 Patterning of Al-Membrane}

\begin{tabular}{|c|c|c|}
\hline Step & Process & \\
\hline 55 & $\begin{array}{l}\text { Cleaning } \mathrm{HNO}_{3} \\
\text { private use } \\
(\# \text { clean104) }\end{array}$ & $\begin{array}{l}\text { NL-CR / WB 9-10 } \\
\mathrm{HNO}_{3}(99 \%) \text { Selectipur: MERCK } \\
\text { - Beaker: } \mathrm{HNO}_{3}(99 \%) \\
\text { - Time: }>10 \text { min } \\
\text { - Quick Dump Rinse: }<0.1 \mu \mathrm{S} \\
\text { - Spin drying }\end{array}$ \\
\hline \multirow[t]{5}{*}{56} & $\begin{array}{l}\text { Standard lithography } \\
\text { Olin 907-17 } \\
(\# l i t h 1002)\end{array}$ & $\begin{array}{l}\text { NL-CR } \\
\text { Continue immediately with } \\
\text { priming the step! }\end{array}$ \\
\hline & Dehydration bake & $\begin{array}{l}\text { NL-CR / WB 21-22 } \\
\text { - Hotplate: } 120^{\circ} \mathrm{C}: 5 \mathrm{~min}\end{array}$ \\
\hline & Priming (HMDS) & $\begin{array}{l}\text { Spincoater } \\
\text { - Spin program: } 4 \text { (4000 rpm, } 30 \mathrm{~s})\end{array}$ \\
\hline & Coating (OLIN 907-17) & $\begin{array}{l}\text { Primus Spinner } \\
\text { - Spin program: } 4(4000 \mathrm{rpm}, 30 \mathrm{~s}, 1.7 \mu \mathrm{m}) \\
\text { - Prebake }\left(95^{\circ} \mathrm{C}\right): 90 \mathrm{~s}\end{array}$ \\
\hline & $\begin{array}{l}\text { Alignment \& Exposure } \\
\text { (Olin 907-17) }\end{array}$ & Electronic Vision Group 620 Mask Aligner \\
\hline
\end{tabular}


- Hg-lamp: $12 \mathrm{~mW} \mathrm{~cm}^{-2}$

- Exposure time: $3.8 \mathrm{~s}$

- Mask: ELECT

Development (Olin 907-17) Developer: OPD4262

- After exposure bake $\left(120^{\circ} \mathrm{C}\right): 60 \mathrm{~s}$

- Time: $30 \mathrm{~s}$ in beaker 1

- Time: 15-30s in beaker 2

- Quick Dump Rinse: $<0.1 \mu \mathrm{S}$

- Spin drying

Postbake (Olin 907-17)

Inspection by

optical microscope
- Hotplate: $120^{\circ} \mathrm{C}: 1 \mathrm{~h}$

NL-CR / Nikon Microscope

Dedicated microscope for lithography inspection

\begin{tabular}{|c|c|c|}
\hline 57 & $\begin{array}{l}\text { Etching of Al } \\
\text { with OPD } 4262 \\
(\# E t c h 091)\end{array}$ & $\begin{array}{l}\text { NL-CR } \\
\text { Etchant: OPD } 4262 \\
\text { Etch-rate: } 14 \mathrm{~nm} / \mathrm{min} \\
\text { Use dummy first! } \\
\text { Maximum etch-time about } 6.5 \mathrm{~min} \\
\text { - Quick Dump Rinse > } 10.5 \mathrm{M} \Omega \\
\text { - Spin drying } \\
\text { Thickness: } \mathbf{8 0 \mathbf { n m }} \\
\text { Etch-time: } \mathbf{6} \mathbf{m i n} \\
\text { \# of dummies: } \mathbf{1}\end{array}$ \\
\hline 58 & $\begin{array}{l}\text { Stripping of polymers } \\
\text { in } \mathrm{HNO}_{3} \text { multipurpose } \\
(\# \text { lith194) }\end{array}$ & $\begin{array}{l}\text { NL-CR-WB6 } \\
\mathrm{HNO}_{3}(100 \%) \\
\text { - Beaker } \mathrm{HNO}_{3}(100 \%):>10 \mathrm{~min} \\
\text { - Quick Dump Rinse: }<0.1 \mu \mathrm{S} \\
\text { - Spin drying }\end{array}$ \\
\hline
\end{tabular}

\section{E.5.16 Backside patterning of SiRN}

\begin{tabular}{lll}
\hline Step & Process & \\
\hline 59 & Standard lithography & NL-CR \\
& Olin $908-35$ & Continue immediately with \\
& $(\# l i t h 1003)$ & priming the step!
\end{tabular}


Dehydration bake

Priming (HMDS)

Coating (OLIN 908-35)

Alignment \& Exposure

(Olin 908-35)

Development (Olin 908-35)

Postbake (Olin 908-35)

Inspection by optical microscope
NL-CR / WB 21-22

- Hotplate: $120^{\circ} \mathrm{C}: 5 \mathrm{~min}$

Spincoater

- Spin program: 4 (4000 rpm, 30s)

Primus Spinner

- Spin program: 4 (4000 rpm, 30s, $3.5 \mu \mathrm{m})$

- Prebake $\left(95^{\circ} \mathrm{C}\right): 120 \mathrm{~s}$

Electronic Vision Group 620 Mask Aligner

- Hg-lamp: $12 \mathrm{~mW} \mathrm{~cm}^{-2}$

- Exposure time: 9s

- Mask: TRENCH

Developer: OPD4262

- After exposure bake $\left(120^{\circ} \mathrm{C}\right): 60 \mathrm{~s}$

- Time: $30 \mathrm{~s}$ in beaker 1

- Time: $15-30$ s in beaker 2

- Quick Dump Rinse: $<0.1 \mu \mathrm{S}$

- Spin drying

- Hotplate: $120^{\circ} \mathrm{C}: 15 \mathrm{~min}$

NL-CR / Nikon Microscope

Dedicated microscope for lithography inspection

\begin{tabular}{|c|c|c|}
\hline 60 & $\begin{array}{l}\text { Plasma etching of } \\
\text { multilayers } \\
(\# \text { etch } 174)\end{array}$ & $\begin{array}{l}\text { NL-CR / Adixen AMS } 100 \mathrm{SE} \\
\text { Program: ... } \\
\text { - Argon flow: } 100 \mathrm{sccm} \\
\text { - } \mathrm{CHF}_{3} \text { flow: } 100 \mathrm{sccm} \\
\text { - ICP: } 1200 \mathrm{~W} \\
\text { - } \mathrm{CCP}: 150 \mathrm{~W} \\
\text { - } \mathrm{SH}: 200 \mathrm{~mm} \\
\text { - APC: } 100 \% \\
\text { - He: } 10 \mathrm{mbar} \\
\text { - Electrode: }-10{ }^{\circ} \mathrm{C} \\
\text { - Etch-rate } \mathrm{Si}: 70-80 \mathrm{~nm} \mathrm{~min}^{-1} \\
\text { - Etch-rate } \mathrm{Olin} \mathrm{PR}: 160 \mathrm{nmmin}^{-1} \\
\text { - Etch-rate } \mathrm{SiO}: 250 \mathrm{~nm} \mathrm{~min}^{-1} \\
\text { - Etch-rate } \mathrm{SiRN}: 300 \mathrm{~nm} \mathrm{~min}^{-1} \\
\text { - Etch-rate } \mathrm{SU}-8: 150 \mathrm{~nm} \mathrm{~min}^{-1}\end{array}$ \\
\hline
\end{tabular}


Thickness: $>1500 \mathrm{~nm}$

Etch-time: $6 \mathrm{~min}$

\# of dummies: 0

\begin{tabular}{|c|c|c|}
\hline 61 & $\begin{array}{l}\text { Stripping of Olin PR by } \\
\text { oxygen plasma (Tepla 300) } \\
\text { (\#lith142) }\end{array}$ & $\begin{array}{l}\text { CR } 125 \text { A / Tepla } 300 \\
\text { Barrel Etcher }(2.45 \mathrm{GHz}) \\
\text { Ultra clean system only (no metals except Al) } \\
\text { - See list with recipes in cleanroom } \\
\text { - } \mathrm{O}_{2} \text { flow: } 200 \mathrm{sccm}(50 \%) \\
\text { - Power: up to } 1000 \mathrm{~W} \\
\text { - Pressure: } 1 \text { mbar }\end{array}$ \\
\hline 62 & $\begin{array}{l}\text { Stripping of polymers } \\
\text { in } \mathrm{HNO}_{3} \text { multipurpose } \\
(\# l i t h 194)\end{array}$ & $\begin{array}{l}\text { NL-CR-WB6 } \\
\mathrm{HNO}_{3}(100 \%) \\
\text { - Beaker } \mathrm{HNO}_{3}(100 \%):>10 \mathrm{~min} \\
\text { - Quick Dump Rinse: <0.1 } \mathrm{HS} \\
\text { - Spin drying }\end{array}$ \\
\hline
\end{tabular}

\section{E.5.17 SU-8 hairs}

\begin{tabular}{|c|c|c|}
\hline Step & Process & \\
\hline \multirow[t]{6}{*}{63} & $\begin{array}{l}\text { Standard lithography } \\
\text { SU-8 } \mathbf{1 0 0} \\
(\# \text { lith1003) }\end{array}$ & NL-CR \\
\hline & Dehydration bake & $\begin{array}{l}\text { NL-CR / WB 21-22 } \\
\text { - Hotplate } 120^{\circ} \mathrm{C}: 5 \mathrm{~min}\end{array}$ \\
\hline & $\begin{array}{l}\text { Coating (SU-8 100) } \\
\text { (Delta 20) }\end{array}$ & SüssMicroTec Spinner Delta 20 \\
\hline & & $\frac{\text { Experimental results: }}{8-1000 \mathrm{rpm}-380 \mu \mathrm{m}}$ \\
\hline & Prebake (SU-8 100) & $\begin{array}{l}\text { - } \text { Start @ } 25^{\circ} \mathrm{C} \\
\text { - } 10 \mathrm{~min} @ 50^{\circ} \mathrm{C} \\
\text { - } 30 \mathrm{~min} @ 65^{\circ} \mathrm{C} \\
\text { - } 4 \mathrm{~h} 30 \mathrm{~min} @ 95^{\circ} \mathrm{C} \\
\text { - } 5{ }^{\circ} \mathrm{C} / 5 \mathrm{~min} \text { down to } 25^{\circ} \mathrm{C}\end{array}$ \\
\hline & $\begin{array}{l}\text { Alignment \& } \\
\text { Exposure (SU-8 100) }\end{array}$ & $\begin{array}{l}\text { Electronic Vision Group } 620 \text { Mask Aligner } \\
\text { - Exposure time: } 140 \mathrm{~s}\end{array}$ \\
\hline
\end{tabular}


- Proximity contact $(>30 \mu \mathrm{m})$

Use scotch tape on the mask and the proximity exposure mode $(>20 \mu \mathrm{m})$ to prevent sticking problems

\section{Mask: HAIR1}

After exposure bake

- Start @ $25^{\circ} \mathrm{C}$

(SU-8 100)

- $10 \min @ 50^{\circ} \mathrm{C}$

- $10 \min @ 65^{\circ} \mathrm{C}$

- $50 \mathrm{~min} @ 75^{\circ} \mathrm{C}$

- $5^{\circ} \mathrm{C} / 5$ min down to $25^{\circ} \mathrm{C}$

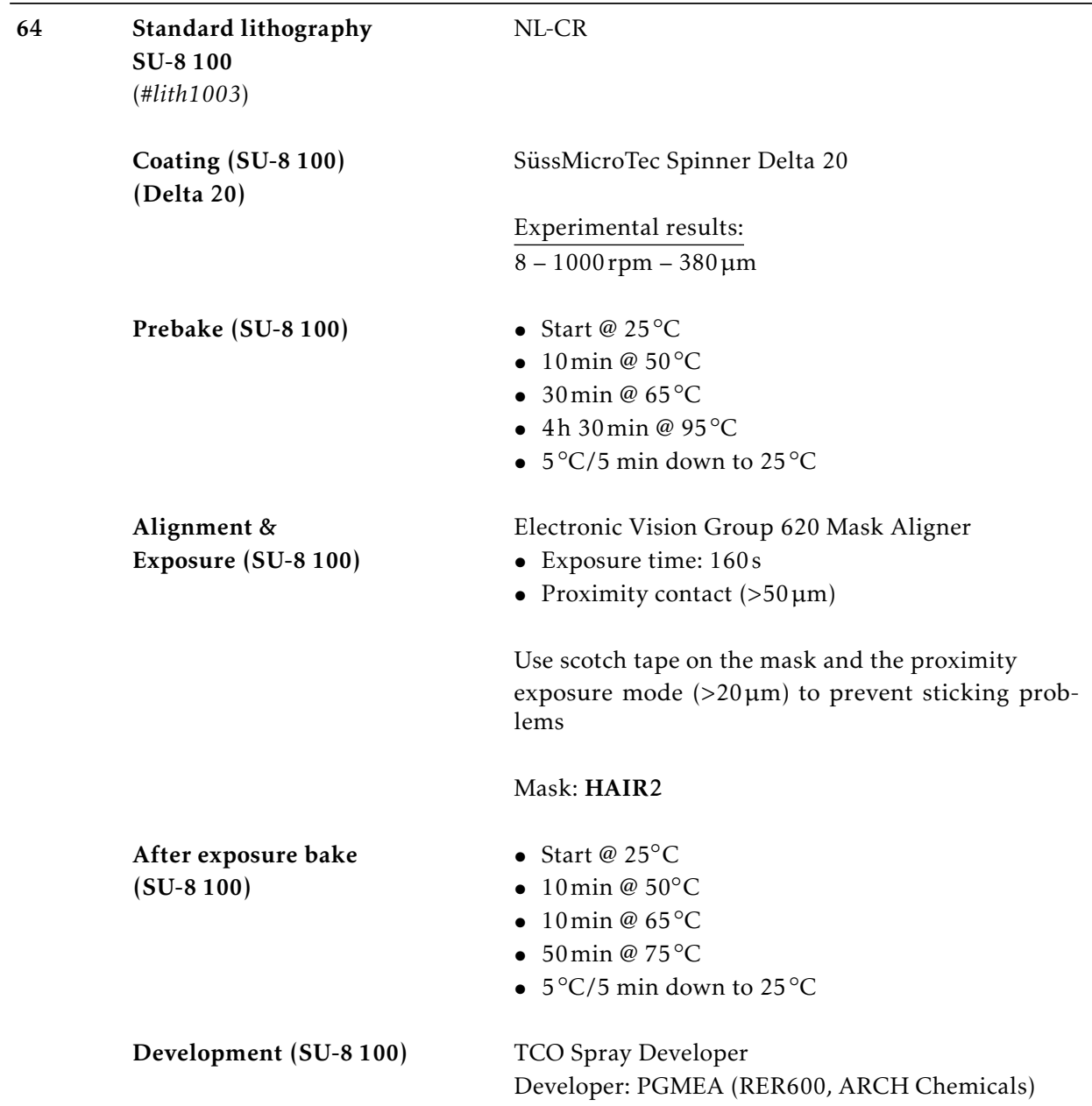


- Time: 40 cycles of $30 \mathrm{~s}$ with spray gun

- Time: 5 s rinse with PGMEA (RER 600) bottle

- Time: $5 \mathrm{~s}$ rinse with IPA

- Spin drying (slow!)

Hard bake (SU-8 100)

- Start@ $25^{\circ} \mathrm{C}$

- $10 \mathrm{~min} @ 50^{\circ} \mathrm{C}$

- $10 \min @ 65^{\circ} \mathrm{C}$

-10min@ $100^{\circ} \mathrm{C}$

- $2 \mathrm{~h} @ 120^{\circ} \mathrm{C}$

- $5^{\circ} \mathrm{C} / 10$ min down to $25^{\circ} \mathrm{C}$

65 Inspection by optical microscope

(\#metro101)
NL-CR / Nikon Microscope

Dedicated microscope for lithography inspection

\section{E.5.18 Sacrificial layer etching}

\begin{tabular}{|c|c|c|}
\hline Step & Process & \\
\hline \multirow[t]{4}{*}{66} & $\begin{array}{l}\text { SLE of poly-Si by } \mathbf{X e F}_{2} \\
(\# E t c h 1300)\end{array}$ & NL-CR \\
\hline & $\begin{array}{l}\text { SLE of polysilicon } \\
\text { by } \mathrm{SF}_{6} \text { (Oxford) }\end{array}$ & $\begin{array}{l}\text { NL-CR Oxford Plasmalab } 100 \mathrm{ICP} \\
\text { - Temperature: } 20^{\circ} \mathrm{C} \\
\text { - } \mathrm{SF}_{6} \text { flow: } 50 \mathrm{sccm} \\
\text { - } \mathrm{CM} \text { pressure: } 10 \mathrm{mtorr} \\
\text { - ICP power: } 600 \mathrm{~W} \\
\text { - CCP power: } 7.5 \mathrm{~W} \\
\text { - He pressure: } 20 \mathrm{mbar} \\
\text { - } \mathrm{V}_{\mathrm{dc}}:-55 \text { to }-40 \mathrm{~V} \\
\text { - Etch-time: } 1 \mathrm{~min}\end{array}$ \\
\hline & $\mathrm{XeF}_{2}$ SLE & $\begin{array}{l}\text { NL-CR / } \mathrm{XeF}_{2} \text { apparatus } \\
\text { - Pressure: } 3 \text { torr } \\
\text { - Cycle time: } 30 \mathrm{~s} \\
\text { - Etch-rate: } 4.9 \mu \mathrm{m} / \text { cycle }\end{array}$ \\
\hline & & Etch-cycles: $\sim 12$ \\
\hline 67 & $\begin{array}{l}\text { Inspection by } \\
\text { optical microscope } \\
\text { (\#metro101) }\end{array}$ & $\begin{array}{l}\text { NL-CR / Nikon Microscope } \\
\text { Dedicated microscope for lithography inspection }\end{array}$ \\
\hline
\end{tabular}




\section{F \\ Process flow for MEMS sliders}

\section{F.1 Introduction}

In this process flow the fabrication process of sensors and devices based upon a single-mask SOI-wafer process is described. The base of the process flow is a silicon-on-insulator (SOI)-wafer. Basically, the sensors and devices are realized by first applying a DRIE-etching step for patterning the device (top) layer of the SOI-wafer. Next, the sensors and devices are released by a vapour HF step, which partially etches the buried oxide (BOX) layer. Furthermore, breaking grooves are added by applying a dicing step before the vapour HF release.

\section{F.2 Specific design properties}

\section{F.2.1 Design rules}

The wafer is divided in sections containing in equally sized squares, each containing a design for the sensor or device. The design rules are shown in table F.1. Every device consists of so-called unit cells with fixed dimensions, as shown in figure F.1. 
Table F.1: Design rules for MEMS sliders.

\begin{tabular}{ll}
\hline Quantity & Value \\
\hline Anchored area & $50 \mu \mathrm{m} \times 50 \mu \mathrm{m}$ \\
Bond-pads & $500 \mu \mathrm{m} \times 500 \mu \mathrm{m}$ \\
Device separation & $100 \mu \mathrm{m}$ (saw width) \\
Total design size & $9 \mathrm{~mm} \times 9 \mathrm{~mm}$ \\
Effective design area & $8 \mathrm{~mm} \times 8 \mathrm{~mm}$ \\
Feature size & $>3 \mu \mathrm{m}$ \\
Beam thickness & $>3.5 \mu \mathrm{m}$ \\
Gap (BOX thickness) & $2 \mu \mathrm{m}$ \\
\hline
\end{tabular}

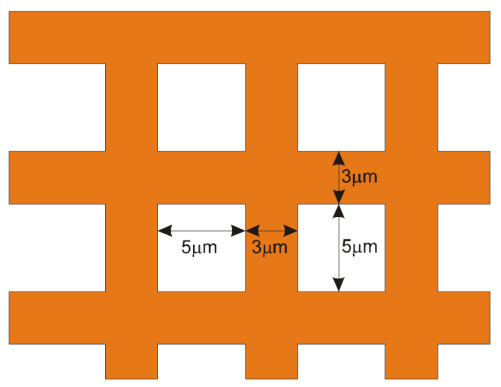

(a) Dimensions of a unit-cell.

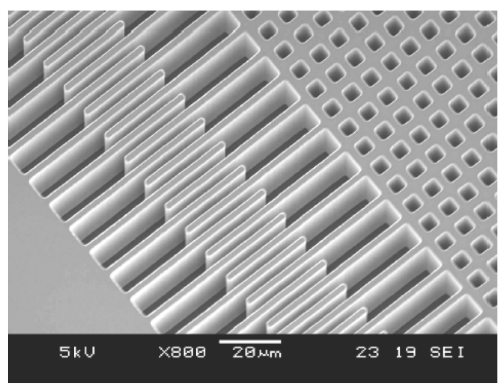

(b) SEM-picture of unit-cells.

Figure F.1: Unit-cell for release of a 'shuttle' (all under-etched) in the device layer.

Table F.2: Mask overview for fabrication of MEMS sliders.

\begin{tabular}{lll}
\hline$\#$ & Name & Description \\
\hline $\mathbf{1}$ & DRIE & Patterning of the device layer for sensors and devices \\
\hline
\end{tabular}

\section{F.2.2 Masks}

The only mask DRIE contains the pattern of the sensors and devices, which are patterned into the device (top) layer (table F.2). 


\section{F.3 Process outline}

Step Process Cross-section after process

- Substrate selection

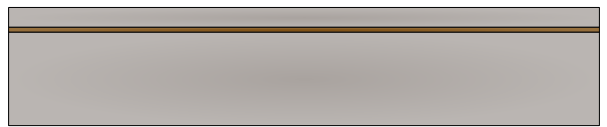

II - Wafer cleaning

- Patterning of device (Si) layer

- Measurement/inspection

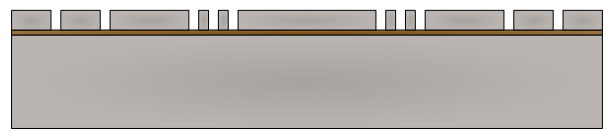

III

- Protection of device layer

- Dicing frontside for breaking grooves

- Removal of protection

- Measurement/inspection

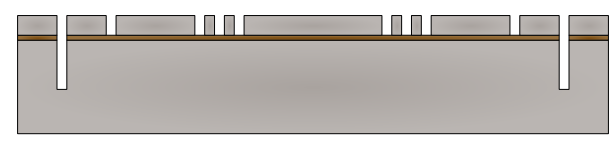

IV

- Release by vapour HF

- Measurement/inspection

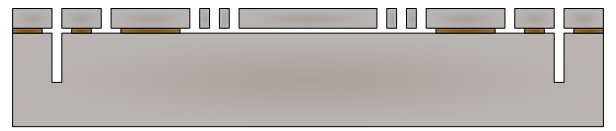

\section{F.4 Process parameters}

\section{F.4.1 Mask inspection}

\begin{tabular}{lll}
\hline Step & Process & \\
\hline $\mathbf{1}$ & $\begin{array}{l}\text { Mask inspection } \\
\text { (\#char001) }\end{array}$ & CR NL / Nikon Microscope \\
& Use form/logbook
\end{tabular}




\section{F.4.2 Substrate selection}

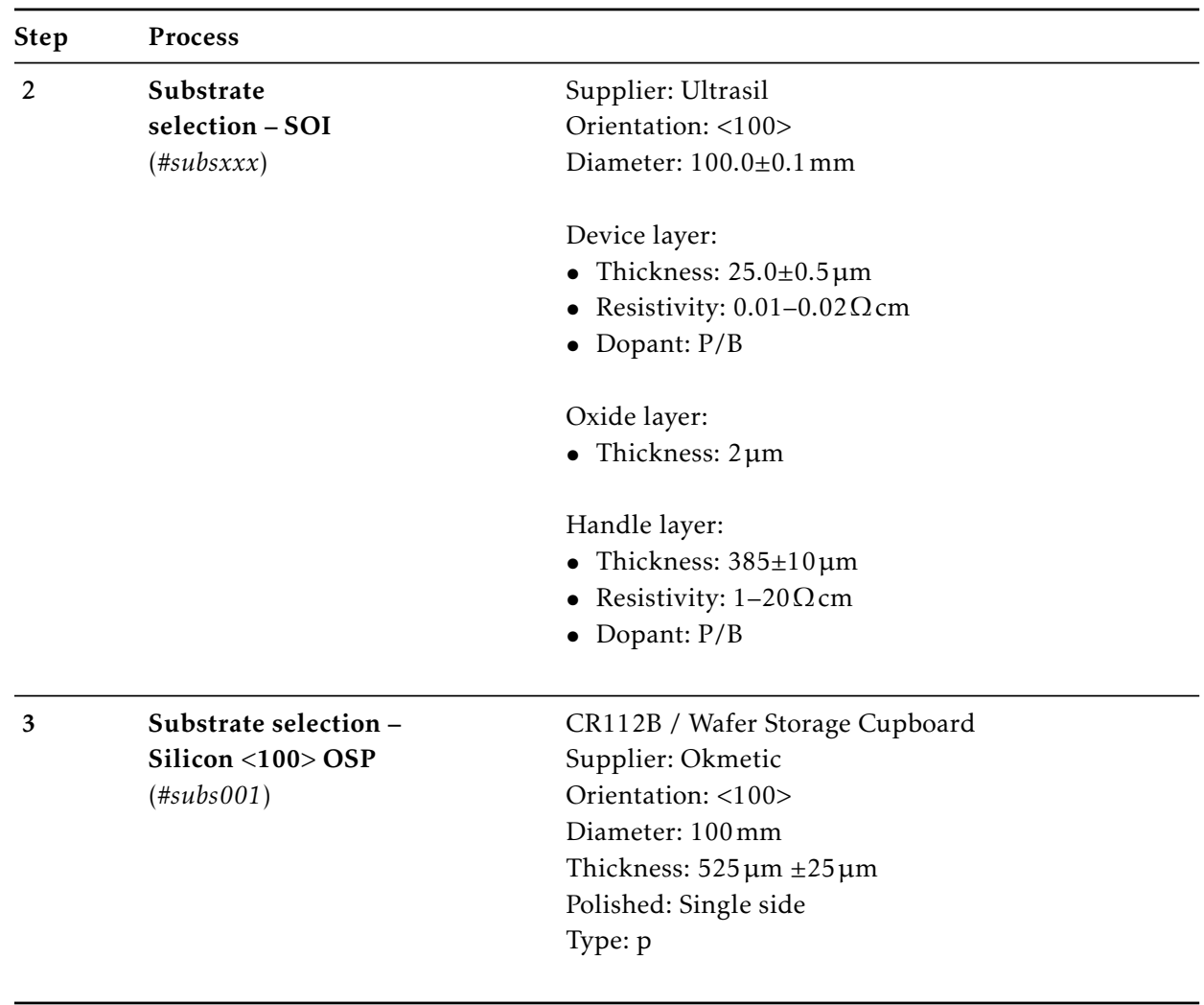

\section{F.4.3 Patterning of the device layer - SOI}

\begin{tabular}{|c|c|c|}
\hline Step & Process & \\
\hline 4 & $\begin{array}{l}\text { Standard cleaning } \mathbf{H N O}_{3} \\
(\# \text { clean } 1001)\end{array}$ & $\begin{array}{l}\text { NL-CR-WB14 } \\
\mathrm{HNO}_{3}(99 \%) \text { Selectiepur: MERCK } \\
\mathrm{HNO}_{3}(69 \%) \text { VLSI: MERCK } \\
\text { - Beaker 1: fumic } \mathrm{HNO}_{3}(99 \%), 5 \mathrm{~min} \\
\text { - Beaker 2: fumic } \mathrm{HNO}_{3}(99 \%), 5 \mathrm{~min} \\
\text { - Quick Dump Rinse: <0.1 } \mu \mathrm{S} \\
\text { - Beaker 3: boiling }\left(95^{\circ} \mathrm{C}\right) \mathrm{HNO} 3(69 \%), 10 \mathrm{~min} \\
\text { - Quick Dump Rinse: }<0.1 \mu \mathrm{S} \\
\text { - Spin drying }\end{array}$ \\
\hline 5 & $\begin{array}{l}\text { Standard lithography } \\
\text { Olin } 907-17\end{array}$ & $\begin{array}{l}\text { NL-CR } \\
\text { Continue immediately with }\end{array}$ \\
\hline
\end{tabular}


(\#lith1002)

Dehydration bake

Priming (HMDS)

Coating (OLIN 907-17)

Alignment \& Exposure

(Olin 907-17)

Development (Olin 907-17)

Postbake (Olin 907-17)

Inspection by

optical microscope priming the step!

NL-CR / WB 21-22

- Hotplate: $120^{\circ} \mathrm{C}: 5 \mathrm{~min}$

Spincoater

- Spin program: 4 (4000 rpm, 30 s)

Primus Spinner

- Spin program: $4(4000 \mathrm{rpm}, 30 \mathrm{~s}, 1.7 \mu \mathrm{m})$

- Prebake $\left(95^{\circ} \mathrm{C}\right): 90 \mathrm{~s}$

Electronic Vision Group 620 Mask Aligner

- Hg-lamp: $12 \mathrm{~mW} \mathrm{~cm}^{-2}$

- Exposure time: $3.8 \mathrm{~s}$

- Mask: DRIE

Developer: OPD4262

- After exposure bake $\left(120^{\circ} \mathrm{C}\right): 60 \mathrm{~s}$

- Time: $30 \mathrm{~s}$ in beaker 1

- Time: $15-30$ s in beaker 2

- Quick Dump Rinse: $<0.1 \mu \mathrm{S}$

- Spin drying

- Hotplate: $120^{\circ} \mathrm{C}: 0 \mathrm{~min}$

NL-CR / Nikon Microscope

Dedicated microscope for lithography inspection
$6 \quad$ Inspection by

optical microscope

(\#metro101)
NL-CR / Nikon Microscope

Dedicated microscope for lithography inspection
$7 \quad$ Plasma etching of Si

B-ARCE TWIN-1

(\#etch175)

\section{NL-CR / Adixen AMS 100 SE}

Aspect Ratio Controlled Etching (reducing RIE-lag) Comb drive layouts (window range $3-40 \mu \mathrm{m}$, depth 25-40 $\mu \mathrm{m}$ )

Mask: OLIN 907-17 (no bake)

- $\mathrm{SF}_{6}$ flow: $400 \mathrm{sccm}$

- $\mathrm{C}_{4} \mathrm{~F}_{8}$ flow: $280 \mathrm{sccm}$

- Time: $5 / 2 \mathrm{~s}$

- Priority: $2 / 1$ 
- ICP: $1500 \mathrm{~W}$

- CCP (pulsed LF): $100 \mathrm{~W}$

- On/off: $10 / 90 \mathrm{~ms}$

- SH: $200 \mathrm{~mm}$

- APC: $100 \%$

- He: $10 \mathrm{mbar}$

- Electrode: $0^{\circ} \mathrm{C}$

- Etch-rate Si: $\pm 1-5 \mu \mathrm{mmin}^{-1}$

- Etch-rate PR: $\pm 25-50 \mathrm{~nm} \mathrm{~min}^{-1}$

Thickness: $25 \mu \mathrm{m}$

Etch-time: $\sim 9 \mathrm{~min}$

\# of dummies: 5

\begin{tabular}{lll}
\hline 8 & $\begin{array}{l}\text { Inspection by } \\
\text { optical microscope } \\
(\# m e t r o 101)\end{array}$ & NL-CR / Nikon Microscope \\
& Dedicated microscope for lithography inspection
\end{tabular}

(\#metro101)

\begin{tabular}{|c|c|c|}
\hline 9 & $\begin{array}{l}\text { SEM inspection } \\
\text { (\#metro103) }\end{array}$ & $\begin{array}{l}\text { NL-CR / SEM } \\
\text { - JEOL JSM } 5610 \text { (cleanroom) } \\
\text { - JEOL JSM } 6380 \text { (cleanroom) } \\
\text { - High resolution SEM LEO (Mark Smithers) }\end{array}$ \\
\hline 10 & $\begin{array}{l}\text { Standard cleaning } \\
\text { after DRIE } \\
\text { (\#clean1008) } \\
\text { Stripping of Olin PR by } \\
\text { oxygen plasma (Tepla } \mathbf{3 0 0})\end{array}$ & $\begin{array}{l}\text { CR-NL } \\
\text { Removal of Olin PR and } \\
\text { FluoroCarbon residue (metal free) } \\
\text { CR-NL / Tepla } 300 \\
\text { Barrel Etcher }(2.45 \mathrm{GHz}) \\
\text { Ultra clean system only (no metals except Al) } \\
\text { - See list with recipes in cleanroom } \\
\text { - O flow: } 200 \text { sccm }(50 \%) \\
\text { - Power: up to } 1000 \mathrm{~W} \\
\text { - Pressure: } 1 \text { mbar } \\
\text { - Time: } 20 \text { min }\end{array}$ \\
\hline & $\begin{array}{l}\text { Stripping resist/cleaning } \\
\text { in "Piranha" private use }\end{array}$ & $\begin{array}{l}\mathrm{NL}-\mathrm{CR} / \mathrm{WB} 9 \\
\mathrm{H}_{2} \mathrm{SO}_{4}: \mathrm{H}_{2} \mathrm{O}_{2}(3: 1) \text { vol\% } \\
\text { - Add } \mathrm{H}_{2} \mathrm{O}_{2} \text { slowly(!) to } \mathrm{H}_{2} \mathrm{SO}_{4} \text {; exothermic process! } \\
\text { - Adjust the hotplate temperature to } 85^{\circ} \mathrm{C} \text {, the } \\
\text { temperature will increase to } 130^{\circ} \mathrm{C} \\
\text { - Cleaning temperature: } 130^{\circ} \mathrm{C} \\
\text { - Time: } 20 \text { min } \\
\text { - Quick Dump Rinse: }<0.1 \mu \mathrm{S}\end{array}$ \\
\hline
\end{tabular}


- Spin drying

Dry oxidation of Silicon at $800^{\circ} \mathrm{C}$

Etching in HF $1 \%$ (private use)
NL-CR / Furnace B3

Standby temperature: $800^{\circ} \mathrm{C}$

- Program: 0X800C

- Temperature: $800^{\circ} \mathrm{C}$

- Gas: $\mathrm{O}_{2}$

- Flow: $2 \mathrm{~L} \mathrm{~min}^{-1}$

- Time: $30 \mathrm{~min}$

NL-CR / WB 9

Use private beaker HF $1 \%$ standard

- Time: 10 min (etch until hydrofobic surface)

- Quick Dump Rinse: $<0.1 \mu S$

- Spin drying

Dedicated microscope for lithography inspection

\begin{tabular}{lll}
\hline 11 & $\begin{array}{l}\text { Inspection by } \\
\text { optical microscope } \\
(\# m e t r o 101)\end{array}$ & $\begin{array}{l}\text { NL-CR / Nikon Microscope } \\
\text { Dedicated microscope for lithography inspection }\end{array}$ \\
\hline 12 & $\begin{array}{l}\text { SEM inspection } \\
\text { (\#metro103) }\end{array}$ & NL-CR / SEM \\
& - JEOL JSM 5610 (cleanroom) \\
& - JEOL JSM 6380 (cleanroom) \\
& - High resolution SEM LEO (Mark Smithers)
\end{tabular}

\section{F.4.4 Dicing for breaking grooves}

\begin{tabular}{lll}
\hline Step & Process & \\
\hline $\mathbf{1 3}$ & $\begin{array}{l}\text { Lithography - } \\
\text { Coating Olin 908-35 } \\
\text { (\#lith006) }\end{array}$ & $\begin{array}{l}\text { NL-CR-WB21 / Primus coater } \\
\text { Hotplate } 95^{\circ} \mathrm{C}\end{array}$ \\
& Olin $908-35$ \\
& Spin program: $\mathrm{x}(500 \mathrm{rpm}, 45 \mathrm{~s})$ \\
\hline $\mathbf{1 4}$ & $\begin{array}{l}\text { Lithography - } \\
\text { Postbake standard } \\
\text { (\#lith009) }\end{array}$ & NL-CR-WB21 \\
& $\begin{array}{l}\text { Hotplate } 95^{\circ} \mathrm{C} \\
\mathbf{1 5}\end{array}$ & $\begin{array}{l}\text { Dicing of a silicon wafer } 3 \text { min } \\
\text { (\#back101) }\end{array}$ \\
\hline
\end{tabular}




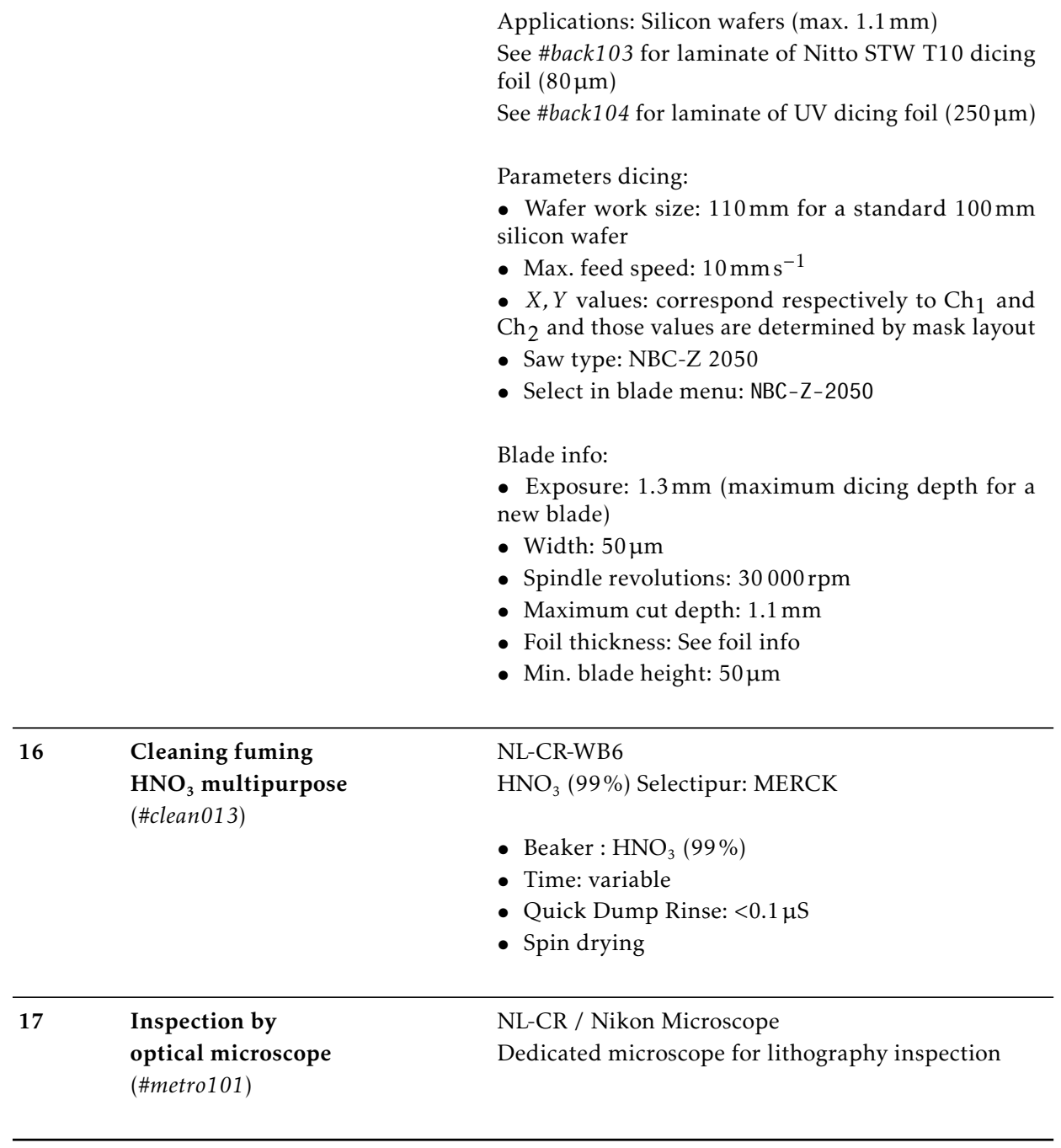

\section{F.4.5 Release by vapour HF}

\begin{tabular}{lll}
\hline Step & Process & \\
\hline 18 & Lithography - & NL-CR-WB21 \\
& Postbake standard & \\
& $(\#$ lith009) & Hotplate $120^{\circ} \mathrm{C}$ \\
& & - Time: $10 \mathrm{~min}$ \\
& \\
\hline
\end{tabular}


- Temperature: $35^{\circ} \mathrm{C}$

- Etch-rate thermal $\mathrm{SiO}_{2}: \pm 110 \mathrm{~nm} \mathrm{~min}^{-1} @ 35^{\circ} \mathrm{C}$

Thickness: $5 \mu \mathrm{m}$

Etch-time: 20-40 min

\# of dummies: 1

20 Inspection by optical microscope

(\#metro101)
NL-CR / Nikon Microscope

Dedicated microscope for lithography inspection 


\section{Publications}

\section{Journal articles}

Droogendijk, H., de Boer, M. J., Sanders, R. G. P., and Krijnen, G. J. M. (2014). A biomimetic accelerometer inspired by the cricket's clavate hair. J. R. Soc. Interface, submitted.

Droogendijk, H., de Boer, M. J., Sanders, R. G. P., and Krijnen, G. J. M. (2014). Advantages of electrostatic spring hardening in biomimetic hair flow sensors. New J. Phys., submitted.

Droogendijk, H., Dagamseh, A. M. K., Sanders, R. G. P., Yntema, D. R., and Krijnen, G. J. M. (2014). Characterization of bio-inspired hair flow sensors: techniques to measure the response for both flow and pressure. Meas. Sci. Technol., submitted.

Droogendijk, H., Bruinink, C. M., Sanders, R. G. P., Dagamseh, A. M. K., Wiegerink, R. J., and Krijnen, G. J. M. (2012). Improving the performance of biomimetic hair-flow sensors by electrostatic spring softening. J. Micromech. Microeng., 22(6):065026.

Droogendijk, H., Bruinink, C. M., Sanders, R. G. P., and Krijnen, G. J. M. (2011). Non-resonant parametric amplification in biomimetic hair flow sensors: Selective gain and tunable filtering. Appl. Phys. Lett., 99(21):213503.

Droogendijk, H., Groenesteijn, J., Haneveld, J., Sanders, R. G. P., Wiegerink, R. J., Lammerink, T. S. J., Lötters, J. C., and Krijnen, G. J. M. (2012). Parametric excitation of a micro Coriolis mass flow sensor. Appl. Phys. Lett., 101(22):223511.

Droogendijk, H., Casas, J., Steinmann, T., and Krijnen, G. J. M. (2014). Performance assessment of bio-inspired systems: flow sensing MEMS hairs. Nat. Commun., in preparation. 
Droogendijk, H., de Boer, M. J., Sanders, R. G. P., and Krijnen, G. J. M. (2014). Stochastic resonance in a voltage-controlled micromechanical slider. New J. Phys., submitted.

Droogendijk, H., Brookhuis, R. A., de Boer, M. J., Sanders, R. G. P., and Krijnen, G. J. M. (2014). Towards a biomimetic gyroscope inspired by the fly's haltere using MEMS technology. J. R. Soc. Interface, submitted.

Droogendijk, H., Bruinink, C. M., Sanders, R. G. P., and Krijnen, G. J. M. (2013). Tunable sensor response by voltage-control in biomimetic hair flow sensors. Micromachines, 4(1):116-127.

Droogendijk, H., Sanders, R. G. P., and Krijnen, G. J. M. (2013). Uncovering signals from measurement noise by electro mechanical amplitude modulation. New. J. Phys., 15:053029.

Brookhuis, R. A., Droogendijk, H., de Boer, M. J., Sanders, R. G. P., Lammerink, T. S. J., Wiegerink, R. J., and Krijnen, G. J. M. (2014). Six-axis force-torque sensor with a large range for biomechanical applications. J. Micromech. Microeng., accepted.

Dagamseh, A. M. K., Bruinink, C. M., Wiegerink, R. J., Lammerink, T. S. J., Droogendijk, H., and Krijnen, G. J. M. (2013). Interfacing of differentialcapacitive biomimetic hair flow-sensors for optimal sensitivity. J. Micromech. Microeng., 23:035010.

Groenesteijn, J., Droogendijk, H., Wiegerink, R. J., Lammerink, T. S. J., Lötters, J. C., Sanders, R. G. P., and Krijnen, G. J. M. (2014). Parametric amplification in a micro Coriolis mass flow sensor. J. Phys. D: Appl. Phys., in preparation.

Krijnen, G., Droogendijk, H., Dagamseh, A., Jaganatharaja, R., and Casas, J. (2013). Imitating the cricket cercal system: The beauty of the beast with a twist of the engineer. Adv. Sci. Tech., 84:19-28.

\section{Conference contributions}

Droogendijk, H., Bruinink, C. M., Sanders, R. G. P., and Krijnen, G. J. M. (2012). Application of electro mechanical stiffness modulation in biomimetic hair flow sensors. In Proc. MEMS 2012, pp. 531-534, Paris, France. 
Droogendijk, H., Brookhuis, R. A., de Boer, M. J., Sanders, R. G. P., and Krijnen, G. J. M. (2012). Design and fabrication of a biomimetic gyroscope inspired by the fly's haltere. In Proc. IEEE Sensors 2012, pp. 1400-1403, Taipei, Taiwan.

Droogendijk, H., de Boer, M. J., Sanders, R. G. P., and Krijnen, G. J. M. (2013). Design, fabrication and characterisation of a biomimetic accelerometer inspired by the cricket's clavate hair. In Proc. IEEE Sensors 2013, pp. 167-170, Baltimore, MD, United States.

Droogendijk, H., Bruinink, C. M., Sanders, R. G. P., Siebelder, O. G., and Krijnen, G. J. M. (2011). Lowering the sensory threshold and enhancing the responsivity of biomimetic hair flow sensors by electrostatic spring softening. In Proc. IEEE Sensors 2011, pp. 829-832, Limerick, Ireland.

Droogendijk, H., Bruinink, C. M., Sanders, R. G. P., and Krijnen, G. J. M. (2011). Non-degenerate parametric amplification and filtering in biomimetic hair flow sensors. In Proc. Transducers'11, pp. 2038-2041, Beijing, China.

Droogendijk, H., Dagamseh, A. M. K., Yntema, D. R., Sanders, R. G. P., and Krijnen, G. J. M. (2011). On the use of various oscillatory air flow fields for characterization of biomimetic hair flow sensors. In Proc. Flow Sensing in Air and Water, p. 62, Bonn, Germany.

Droogendijk, H. and Krijnen, G. J. M. (2010). Simulation studies of parametric amplification in bio-inspired flow sensors. In Proc. MME 2010, pp. 149-152, Enschede, The Netherlands.

Droogendijk, H., de Boer, M. J., Brookhuis, R. A., Sanders, R. G. P., and Krijnen, G. J. M. (2013). Stochastic resonance in a voltage-controlled MEMS-slider: Increasing the signal-to-noise ratio with noise. In Proc. Transducers'13, pp. 1707-1710, Barcelona, Spain.

Dagamseh, A. M. K., Bruinink, C. M., Droogendijk, H., Wiegerink, R. J., Lammerink, T. S. J., and Krijnen, G. J. M. (2010). Engineering of biomimetic hair-flow sensor arrays dedicated to high-resolution flow field measurements. In Proc. IEEE Sensors 2010, pp. 2251-2254, Waikoloa, HI, United States.

Groenesteijn, J., Droogendijk, H., de Boer, M. J., Sanders, R. G. P., Wiegerink, R. J., and Krijnen, G. J. M. (2014). An angular acceleration sensor inspired by the vestibular system with a fully circular fluid-channel and thermal read-out. In Proc. MEMS 2014, pp. 696-699, San Francisco, CA, United States. 
Groenesteijn, J., Droogendijk, H., Wiegerink, R. J., Lammerink, T. S. J., Lötters, J. C., Sanders, R. G. P., and Krijnen, G. J. M. (2013). Parametric amplification in a micro Coriolis mass flow sensor: reduction of power dissipation without loss of sensitivity. In Proc. IEEE Sensors 2013, pp. 1626-1629, Baltimore, MD, United States.

Haneveld, J., Lammerink, T. S. J., Dijkstra, M. A., Droogendijk, H., de Boer, M. J., and Wiegerink, R. J. (2008). Highly sensitive micro coriolis mass flow sensor. In Proc. MEMS 2008, pp. 920-923, Tucson, AZ, United States.

Jaganatharaja, R. K., Droogendijk, H., Vats, S., Hagedoorn, B., Bruinink, C. M., and Krijnen, G. J. M. (2011). Unraveling the viscosity-mediated coupling effect in biomimetic hair sensor arrays. In Proc. MEMS 2011, pp. 652-655, Cancun, Mexico.

Lötters, J. C., Groenesteijn, J., Dijkstra, M., Droogendijk, H., Lammerink, T. S. J., and Wiegerink, R. J. (2013). Micro coriolis mass flow sensor with extended range for a monopropellant micro propulsion system. In Proc. AMA Conferences 2013, pp. 508-512, Nürnberg, Germany.

\section{Book chapters}

Droogendijk, H. and Krijnen, G. J. M. (2015). Bio-inspired inertial sensors. In Biomimetic technologies: Volume I. Woodhead Publishing.

Krijnen, G. J. M., Droogendijk, H., Dagamseh, A. M. K., Jaganatharaja, R. K., and Casas, J. (2014). Biomimetics and crickets. In Flow Sensing in air and water. Springer.

Krijnen, G. J. M., Droogendijk, H., Steinmann, T., Dagamseh, A. M. K., Jaganatharaja, R. K., and Casas, J. (2014). Crickets as bio-inspiration for MEMSbased flow-sensing. In Handbook of biomimetics and bioinspiration. World Scientific Publishing Company.

\section{Miscellaneous}

Droogendijk, H. (2012). Biomimetic hair flow sensors. Symposium "Nature of Physics". Groningen, The Netherlands.

Science Daily (2011). Synthetic cricket pricks up its 'ears'. 
Science Daily (2012). Measuring flow using a tiny wobbling tube.

Science Daily (2013). Sensitive flow sensor: Hair sensor uncovers hidden signals. van der Heijden, M. (2011). Door krekels geïnspireerde chips. NRC. Wetenschapskatern.

Wetenschap 24 (2011). Blij met een dooie mug. Hoe?Zo! Radio. 
MISCELLANEOUS 


\section{Samenvatting}

In de natuur zijn verschillende mechanosensoren te vinden die uitgerust zijn met haarachtige structuren. Met deze sensoren worden diverse fysische grootheden gemeten, zoals versnelling, luchtstroming, rotatiesnelheid, balans en infraroodlicht. Zo gebruikt de krekel filoremische haren om laagfrequente luchtstromingen te meten en daarmee informatie te verkrijgen over zijn omgeving om bijvoorbeeld aanvallen van onder andere predators te ontwijken. Deze haarsensoren zijn in staat om luchtstromingen te meten vanaf $30 \mu \mathrm{m} \mathrm{s}^{-1}$ en gebruiken hierbij de hoeveelheid energie die vergelijkbaar is met die van thermische ruis. Met deze haarsensoren als uitgangspunt zijn — met gebruikmaking van de technologie MEMS (micro-elektromechanische systemen) - haar-gebaseerde stromingssensoren met capacitieve uitlezing ontwikkeld voor het meten van kleine harmonische luchtstromingen. Om de prestaties van deze sensoren vast te stellen zijn drie verschillende opstellingen gebruikt. Elk van deze opstellingen heeft specifieke eigenschappen qua frequentiebereik, drukafhankelijkheid en bandbreedte. Door de informatie verkregen met deze opstellingen te combineren zijn belangrijke nieuwe inzichten in het functioneren van de haarsensoren ontstaan.

Om de prestaties van de ontwikkelde haarsensoren te verbeteren wordt de aard van energiebufferende tweepoorttransducenten gebruikt om elektrostatische veerverslapping te bewerkstelligen. Door het toepassen van een gelijkspanning op de capacitieve structuren in onze stromingssensoren is zowel een grotere responsiviteit in de bandbreedte van de sensor als een lagere meetdrempel voor de luchtsnelheid te verkrijgen. Wanneer deze gelijkspanning wordt veranderd in een wisselspanning, is niet-resonante parametrische versterking en filtering in de haarstromingssensoren aan te tonen. Door het kiezen van geschikte waardes voor deze spanning is het mogelijk selectieve versterking en filtering te behalen. In het geval een wisselspanning met een voldoende hoge frequentie wordt toegepast ten opzichte van de frequentie van de luchtstroming wordt omhoog-conversie van de stromingsinformatie door de implicaties van EMAM (elektromechanische amplitude modulatie) bereikt. Er wordt aangetoond dat EMAM kan helpen om de kwaliteit van metingen voor laagfrequente luchtstromingen te verbeteren indien 
de gebruikte meetopstelling beperkingen kent. Deze methode kan niet alleen op harmonische signalen worden toegepast, maar ook op andere soorten signalen, zoals transiënten.

Onder bepaalde omstandigheden kan aan een systeem ruis worden toegevoegd om door middel van het concept SR (stochastische resonantie) de signaalruisverhouding te verhogen. Dit concept wordt aangetoond in een spanning-gestuurd MEMS-schuifsysteem, waar de signaalruisverhouding wordt verhoogd door het toevoegen van witte ruis. Het concept SR is geïmplementeerd door de sterkte van positieafhankelijke capacitieve putten met een gelijkspanning te regelen, het systeem in een duw-trek modus elektrostatisch te actueren en een slimme hoeveelheid ruis aan de actuatiestructuren toe te voegen. Er wordt aangetoond dat het gebruik van SR het mogelijk maakt op subdrempelkrachten te detecteren en dat de bandbreedte van de toegevoegde ruis een groot effect heeft op de vereiste hoeveelheid optimale ruis.

Daarnaast zijn er drie verschillende types bio-geïnspireerde inertiale sensoren ontwikkeld. Allereerst is er een biomimetische versnellingsopnemer gerealiseerd met behulp van oppervlaktemicrotechnologie en SU-8 lithografie, geïnspireerd door het clavate haarsysteem van de krekel. Ten tweede is er een biomimetische gyroscoop ontworpen, gefabriceerd en deels gekarakteriseerd. Deze haargebaseerde gyroscoop is geïnspireerd door de halteres van de vlieg en is cardanisch opgehangen. Als derde is een hoekversnellingsopnemer gebaseerd op de semicirculaire kanalen van het vestibulair systeem ontwikkeld. Deze versnellingsopnemer bestaat uit een met water gevulde buis, waarin de vloeistofsnelheid thermisch wordt gemeten en een maat is voor de externe hoekversnelling. Voor al deze sensoren worden de benodigde modellen gepresenteerd en worden richtlijnen voor het ontwerp afgeleid. Daarnaast worden de prestaties vergeleken met hun biologische tegenhangers en wordt de biomimetische potentie van elke sensor behandeld.

Om de prestaties van onze MEMS haarstromingssensoren te kwantificeren en die vervolgens te vergelijken met hun biologische tegenhanger, worden vijf onafhankelijke metrieken en een cijfer van verdienste beschreven, gemodelleerd en geëvalueerd voor zowel de krekel als de MEMS sensoren. In algemene zin presteert de krekel niet alleen beter dan de MEMS sensoren, maar opereren diens haarsensoren ook in de buurt van de fysische limieten. Deze resultaten benadrukken het intrigerende onderzoek naar bio-geïnspireerde sensoren om te leren van de natuur. 


\section{Woord van dank}

$\mathrm{Na}$ ruim vier jaar hard werken, onderzoek doen en publiceren ligt hier dan het resultaat waardoor ik mij in de rij van doctoren mag scharen. Dat tijd een relatief begrip is, wordt door het verloop van mijn promotietraject maar weer eens bevestigd. Ik weet nog goed hoe het allemaal begon met het inrichten van mijn bureau in een kamer op vloer 7 van gebouw Hogekamp. Een bijzondere en bewogen tijd, waarin ik met veel inzet heb toegewerkt naar dit tastbare eindresultaat. En ook al ben ik degene die door dit proefschrift zal promoveren, er zijn veel mensen die op een zekere manier hebben bijgedragen aan het resultaat en ik wil hen dan ook graag noemen en bedanken.

Zonder promotor geen promotie. Gijs, jij hebt voor mij de afgelopen jaren ontelbaar veel functies gehad. Je was niet alleen mijn promotor, maar je was ook mijn begeleider, inspirator, criticus, grote baas, reviewer, aanzetter, reisgenoot, en noem maar op. Ik heb erg genoten en ook geleerd van de vele overleggen en discussies over mijn onderzoek en daarbij behorende experimenten, meetresultaten, ideeën en publicaties. Ik waardeer het ontzettend dat je me de vrijheid hebt gegeven om flink buiten de paden van de projectomschrijving van work package 3 van het BioEARS-project te gaan. Aanvankelijk zou mijn onderzoek zich richten op parametrische versterking en stochastische resonantie in haarstromingssensoren. Maar het werd veel meer, het werd ook onderzoek naar vermogensoptimalisatie in haarsensoren, het zorgvuldig beschrijven en demonstreren van het concept EMAM, haar-gebaseerde versnellingssensoren en gyroscopen, kijken of we sprinkhaan-geïnspireerde oren kunnen maken, een hoekversnellingssensor maken geïnspireerd door het evenwichtsorgaan, en het gave experiment of onze haartjes een beetje robuust zijn door ze in een windtunnel te plaatsen (waaruit is gebleken dat ze een orkaan prima doorstaan).

Ook veel dank aan Chrix en Meint voor hun rol en bijdrage in de $\mathrm{MESA}^{+}$ cleanroom. Het proces voor het maken van de haarsensoren is voorafgaand aan mijn promotie-traject door Chrix zorgvuldig uitgewerkt, waardoor ik een enorme springplank had om mijn sensoren door de cleanroom te trekken. Geen makkelijk proces overigens, want de laatste versie van het haartjesproces telt toch al snel 
acht maskers, en een veelvoud aan depositie- en etsstappen. Dit houdt in dat je zo drie maanden in de cleanroom staat, waarbij het hopen is dat alles maar goed is gegaan. Chrix, bedankt voor het helpen met het uitbreken van mijn chips uit de wafer, wat misschien wel het spannendste onderdeel van het hele fabriceren is. Meint, we hebben veel vruchtbare en gezellige overleggen gehad over het zijn van 'micromachinist' in de cleanroom. Naast de haartjes hebben we ook het zogenoemde $\mathrm{H}$-design proces verder ontwikkeld, wat heeft geleid tot de schuifstructuren die in dit proefschrift staan beschreven. Het is fijn om uit een brok ervaring te mogen putten, evenals iemand te hebben die de Adixen SE wél mag bedienen.

Pino (Remco S.), we hebben de afgelopen jaren veel tijd doorgebracht achter de Polytec MSA-400. Het dure maar geweldige optische systeem in de groep, waarmee we veel in-het-vlak, uit-het-vlak en topografische metingen hebben gedaan. Jouw expertise hierin heeft geleid tot vele mooie en zinvolle metingen, met menig publicatie tot gevolg. Ik vergeet ook niet je gesleutel aan de SMAC-piston, waarmee we hebben kunnen laten zien dat EMAM ook kan worden toegepast voor transient-flows, evenals het videometen voor mijn schuifstructuren, waarmee ik de bewegingen van deze structuren in kaart kon brengen.

Binnen het BioEARS-project hebben niet alleen Gijs, Chrix, Pino en Meint gewerkt, maar ook anderen. Ik bedank ook hen hartelijk voor hun bijdrages, waar ik op velerlei manieren baat bij heb gehad. Ram, Ahmad en Nima, jullie hebben allen als promovendus gewerkt aan biomimetische haarsensoren. Bedankt voor de prettige samenwerking en de mooie wetenschappelijke resultaten die zijn geboekt. Remco W., jij hebt, samen met Theo, veel betekend op het gebied van de elektronica voor het meten van de haarrotatie. Ik heb met de elektronica die eigenlijk ontwikkeld was voor de micro Coriolis mass flow sensor veel mooie metingen mogen en kunnen doen, die in diverse publicaties zijn gebruikt. Vitaly, bedankt voor jouw voorwerk op het gebied van parametrische resonantie in de haarsensoren. Was ik in het begin nogal gechoqueerd door de hoeveelheid wiskunde en theorie, nu beschouw ik het als niet meer dan logisch.

Ook een woord van dank aan Jérôme en Thomas. Het is erg boeiend als ingenieurs en biologen met elkaar in gesprek gaan, en helemaal wanneer dat leidt tot één of meerdere publicaties. We hebben de afgelopen jaren hard gewerkt aan een verhaal over hoe gaaf die haarsensoren nu eigenlijk zijn (krekel vs. MEMS), en dit heeft tot vele leuke, verrassende en nieuwe inzichten geleid. Jérôme, we hebben vele constructieve Skype-gesprekken gevoerd, alleen snap ik nog niet helemaal waarom er regelmatig een 'krekel op de lijn' zat.

Robert, we hebben de afgelopen jaren veel met elkaar opgetrokken. Dit begon eigenlijk al met het afstuderen destijds, en sindsdien zijn we op dezelfde voet 
verder gegaan. We zijn in te veel kamers elkaars genoot geweest en hebben hierbij veel vruchtbare discussies gehad, ook al ging het niet altijd ergens over. Dank voor je assistentie als er weer eens een verbinding in de elektronica slecht bleek te zijn, maar ook voor je hulp bij het doorlopen van nieuwe (masker)ontwerpen en het bespreken van meer fundamentele wetenschappelijke kwesties. We zijn erg betrokken geweest bij elkaars onderzoek, waarbij er duidelijk sprake is geweest van een win-winsituatie.

Jarno, bedankt voor de samenwerking op het gebied van de 'buizen'. Het toepassen van (varianten van) mijn parametrische werk op jouw micro Coriolis mass flow sensor blijkt een gouden zet te zijn geweest. Een mooi voorbeeld van samenwerking tussen promovendi, met diverse publicaties als resultaat. Ook het hoofdstuk over de hoekversnellingssensor was zonder onze samenwerking niet tot stand gekomen.

De afgelopen jaren heb ik ook regelmatig mijn koffers mogen pakken voor een conferentie, waarbij ik diverse landen heb bezocht: Duitsland, België, Frankrijk, Ierland, Spanje, China, Taiwan en de Verenigde Staten. Ik noem drie conferenties in het bijzonder. Het bezoek aan China in 2011 voor de Transducers' 11 conferentie in Beijing was mijn eerste bezoek aan het Aziatische continent. Gijs, Joost, Mubassira en Robert, we hebben een leuke tijd gehad. Twee jaar later stond Transducers weer op het programma, maar nu was Barcelona de bestemming. Robert, Rolf en Remco W., we hebben een gezellige en prettige tijd gehad samen in Catalonië. Eind 2013 zijn we met een flinke delegatie (Robert, Jarno, Olti, Remco W. en Gijs) afgereisd naar Baltimore voor IEEE Sensors 2013. Ik kijk met veel genoegen terug op de conferentie en op wat we daar verder hebben beleefd.

Laat ik ook de andere collega's uit de vakgroep Transducers Science and Technology bedanken. Wetenschappelijk staf, technici, secretaresses, promovendi en studenten, allen maken een groep tot wat die is. Ik heb de afgelopen vijf jaar een prachtige tijd gehad: fijne mensen en niets was te gek. We hebben elkaar niet alleen vaak bij de koffiehoek mogen zien, maar ook bij de wekelijkse MicMec-talks en de jaarlijkse TST-outing.

Ook al staat voor het promoveren ongeveer vier jaar, eigenlijk is alles wat er aan voorafgaat (lees: geboorte tot nu) bepalend voor hoe de promotie er uiteindelijk uit zal zien. Het is ook daarom dat ik mijn ouders Jan en Wina heel erg bedank voor hun grote bijdrage (opvoeding, stimulering, noem maar op) aan waar ik nu sta. Ik weet nog dat mijn vader tijdens mijn studie eens zei: "Harmen, heb je al eens gedacht om na je afstuderen te gaan promoveren?" Destijds reageerde ik afwijzend, maar nam het klaarblijkelijk wel mee in de gedachtegang. Uiteindelijk heb ik toch de keuze om te gaan promoveren vol overtuiging gemaakt en is dit proefschrift het tastbare eindresultaat. 
Last, but not least, wil ik mijn grote en liefdevolle dank uitspreken aan mijn vrouw. Klaske, de afgelopen jaren ben jij altijd mijn steun en toeverlaat geweest en heb je me altijd gestimuleerd om het onderste uit de (wetenschappelijke) kan te halen. De afgelopen vier jaar zijn voor ons extra speciaal geweest, omdat in deze tijd ons gezin in samenstelling is verdubbeld met de geboorte van dochter Lieneke (2010) en zoon Ruben (2013). Door een goede balans tussen werk en privé heb ik veel mogen en kunnen meemaken van de eerste jaren van onze kinderen; veel bijzondere momenten die dierbaar en uniek zijn.

Harmen Droogendijk

Enschede, januari 2014 


\section{Biografie}

Harmen Droogendijk werd op vrijdag 11 januari 1985 geboren te Tholen. Hij verhuisde in 1989 naar Anjum en in 1994 naar Workum. Van 1997 tot 2003 doorliep hij het vwo te Sneek (C.S.G. Bogerman). In 2003 begon hij met de bacheloropleiding Elektrotechniek aan de Universiteit Twente, die hij met goed gevolg afrondde (cum laude). In 2007 ging hij verder met een masteropleiding Electrical Engineering aan dezelfde universiteit, met als richting Microsystems and Microelectronics. Zijn afstudeerwerk voerde hij uit bij de vakgroep TST (Transducers Science and Technology) over een MEMS-accelerometer voor geofysische toepassingen. Hiermee rondde hij ook deze opleiding met goed gevolg af (cum laude).

In oktober 2009 ving hij bij de vakgroep TST zijn promotieonderzoek aan binnen het BioEARS-project over het toepassen van niet-lineaire effecten in biomimetische haar-stromingssensoren onder leiding van professor Gijs Krijnen. In 2012 nam hij deel aan traject "Promovendi voor de klas" binnen het $\beta$ XLinxproject van de Universiteit Twente, waarmee hij zijn eerstegraads lesbevoegdheid natuurkunde heeft behaald. 
BIOGRAFIE 